\title{
Risk and uncertainty : classical and modern models for individual decision making
}

Citation for published version (APA):

Zank, H. (1999). Risk and uncertainty : classical and modern models for individual decision making.

[Doctoral Thesis, Maastricht University]. Universiteit Maastricht. https://doi.org/10.26481/dis.19990527hz

Document status and date:

Published: 01/01/1999

DOI:

10.26481/dis.19990527hz

Document Version:

Publisher's PDF, also known as Version of record

\section{Please check the document version of this publication:}

- A submitted manuscript is the version of the article upon submission and before peer-review. There can be important differences between the submitted version and the official published version of record.

People interested in the research are advised to contact the author for the final version of the publication, or visit the DOI to the publisher's website.

- The final author version and the galley proof are versions of the publication after peer review.

- The final published version features the final layout of the paper including the volume, issue and page numbers.

Link to publication

\footnotetext{
General rights rights.

- You may freely distribute the URL identifying the publication in the public portal. please follow below link for the End User Agreement:

www.umlib.nl/taverne-license

Take down policy

If you believe that this document breaches copyright please contact us at:

repository@maastrichtuniversity.nl

providing details and we will investigate your claim.
}

Copyright and moral rights for the publications made accessible in the public portal are retained by the authors and/or other copyright owners and it is a condition of accessing publications that users recognise and abide by the legal requirements associated with these

- Users may download and print one copy of any publication from the public portal for the purpose of private study or research.

- You may not further distribute the material or use it for any profit-making activity or commercial gain

If the publication is distributed under the terms of Article $25 \mathrm{fa}$ of the Dutch Copyright Act, indicated by the "Taverne" license above, 
Risk and Uncertainty:

Classical and Modern Models for Individual Decision Making 



\title{
Risk and Uncertainty:
}

\author{
Classical and Modern Models \\ for Individual Decision Making
}

\section{PROEFSCHRIFT}

ter verkrijging van de gruad van doctor aan de Universiteit Maastricht, op gezag van de Rector Magnificus, Prof. dr. A.C. Nieuwenhuijzen Kruseman, volgens het besluit van het College van Decanen, in het openbaar te verdedigen op donderdag 27 mei 1999 om 16.00 uur

door

Horst Zank 


\section{Promotores:}

Prof. dr. H. J. M. Petcrs

Prof. dr. P. P. Wakker

Beoordelingscommissie:

Prof. dr. F. C. Palm (voorzitter)

Dr. H. Bleichrodt (Erasmus Universiteit Rotterdam)

Dr. J. A. M. Potters (Katholieke Universiteit Nijmegen)

Prof. dr. P. C. Schotman

ISBN 90-5681-06(1-X

NWO Project 510-1-0003 


\section{Contents}

Acknowledgements vii

1 Introduction 1

2 A Unified Derivation of Classical Subjective Expected Utility Models $\begin{array}{ll}\text { through Cardinal Utility } & \mathbf{7}\end{array}$

2.1 Introduction . . . . . . . . . . . . . . . . . . . 7

2.2 The Subjective Expected Itility Model . . . . . . . . . . . . . . . 9

2.3 de Finetti's Approach . . . . . . . . . . . . . . . . . . . . . . . 11

2.4 Anscombe \& Aumann's Approach . . . . . . . . . . . . . . . . . . 13

2.5 von Neumann \& Morgenstern's Approach . . . . . . . . . . . . . . . 16

2.6 Ramsey's Approach: The Equiprobable State Case . . . . . . . . . . . . 17

2.7 Modern SEU Derivations . . . . . . . . . . . . . . . . . . . . 19

2.8 Appendix: Proofs to Chapter 2 . . . . . . . . . . . . . . . 22

3 State Dependent Expected Utility for Savage's State Space 25

3.1 Introduction . . . . . . . . . . . . . . . . . . 25

3.2 State-Dependent Expected Utility . . . . . . . . . . . . . . . . . . . 26

3.3 Absence of General State-Dependent Expected Utility in the Literature . . 29

3.4 Definitions and Preliminary Results . . . . . . . . . . . . . . . . . . . . 31

3.5 Complications for Infinite State Spaces . . . . . . . . . . . . . . . . 33

3.6 State-Dependent Expected Utility for Infinite State Spaces . . . . . . . . . 37

3.7 Deriving an Integral Form under Countable Additivity . . . . . . . . . . . 40

3.8 Applications . . . . . . . . . . . . . . . . . . . . . 42

3.9 Conclusion . . . . . . . . . . . . . . . . . . . . 45

3.10 Extensions and Further Comments . . . . . . . . . . . . . . . . . 46

3.11 Connected Topological Outcome Spaces . . . . . . . . . . . . . . . 47 
3.12 Appendix A: Proofs of Results in Sections 3.1-3.6 . . . . . . . . . 49

3.13 Appendix B: Proofs of Results in Section $3.7 \ldots \ldots . . . . . . .552$

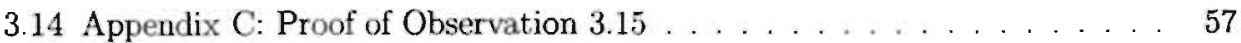

3.15 Appendix D: Proof of Theorems 3.16 and $3.17 \ldots \ldots \ldots$

3.16 Appendix E: Proof of Results in Section $3.8 \ldots \ldots \ldots 2$

4 A Simple Axiomatization of Rank-Dependent Utility and Cumulative Prospect Theory with Constant Proportional Risk Aversion 63

4.1 Introduction . . . . . . . . . . . . . . . . . . 63

4.2 Definitions . . . . . . . . . . . . . . . . . . . . . . 66

4.3 A Preference Characterization . . . . . . . . . . . . . . 68

4.4 Conclusion . . . . . . . . . . . . . . . . . . . . . . . 72

4.5 Appendix: Proofs to Chapter $4 \ldots \ldots$. . . . . . . . . . 72

5 Cumulative Prospect Theory for Parametric and Multiattribute Utilities

5.1 Introduction . . . . . . . . . . . . . . . . . . . . 79

5.2 Cumulative Prospect Theory with Linear/Exponential or Power Utility . . 81

5.3 Cumulative Prospect Theory with Multilinear and with Additive/Multiplicative Utility . . . . . . . . . . . . . . . . . . . . 86

5.4 Appendix: Proofs to Chapter 5 . . . . . . . . . . . . . . . . 89

6 A Class of Methods for Evaluating Multiattribute Utilities for Health $\begin{array}{ll}\text { States } & 101\end{array}$

6.1 Introduction . . . . . . . . . . . . . . . . . . . . 101

6.2 Main Concepts . . . . . . . . . . . . . . . . . . . . . . 102

6.3 A Characterization of Valuations . . . . . . . . . . . . 105

6.4 Marginalism . . . . . . . . . . . . . . . . . . . 108

6.5 The Egalitarian Valuation . . . . . . . . . . . . . . . 110

6.6 Concluding Remarks . . . . . . . . . . . . . . . . 113

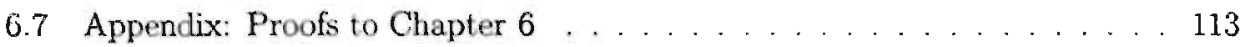

$\begin{array}{ll}\text { References } & 131\end{array}$ 
CONTENTS

Subject Index

Samenvatting

About the Author 



\section{Acknowledgements}

After finishing my Master's thesis in Mathematics at the University of Technology Aachen, my supervisor Bert Jongen introduced me to Hans Peters, who was looking to fill a research position at the Department of Quantitative Economics of the Rijksuniversiteit Limburg. Two weeks later I met Peter Wakker who explained me the possible research topic. Although I did not have experience in the theory of decision making, I decided to take this challenge and to start my Ph.D.-research knowing that the outcome is uncertain. I am indebted to the persons above for providing me with the necessary mathematical tools and for showing me how to use them in Individual Decision Making and in Game Theory. The results in this thesis were first formulated in joint papers with Peter Wakker and with Hans Peters. I thank Louis Gevers and several anonymous referees for giving useful comments improving these results.

From the beginning working at the Quantitative Economics department took place in a pleasant environment. Learning the Dutch language was made easy with the help from Karin, Yolanda and Hans Peters. I am grateful for the friendships arisen during this research period. Thank you André, Bettina, Alain, Bertrand, Thomas, Lars, and Dries. Finally, I wish to thank Dolores for her support right from the start of this Ph.D.-study.

Financial assistance for this project came from The Netherlands Organization for Scientific Research project number 510-1-0003.

Horst Zank 



\section{Chapter 1}

\section{Introduction}

Risk and uncertainty are relevant to many decisions. They are typically present when future results depend on today's decisions and are not entirely forecastable. The prominence of this presence may, however, depend on the type of decision that has to be taken. In order to make the best decision at present a precise analysis of what may happen in the future is needed. Decision theory is the field that investigates individuals' choice behavior, and proposes models to describe this behavior.

The use and the applications of such models to other fields are well-established. In medical care there is uncertainty about the final result of an intervention. When a diagnosis is made, several ways of treatment can be implemented, and physicians have to choose the best of those alternatives. This choice can be difficult, especially when new ways of treatment become available. In the case of life-threatening diseases, a mechanism which makes it possible to choose the treatment that minimizes the risk of dying is called for. It is not surprising that decision models have proved to be very useful here.

In economics, where money plays a central role, maximizing profits is the classical aim of a firm. Before a good is produced, a precise analysis of the market need for this good has to be made. Due to competition it is essential for profit maximization to produce the good such that consumers are willing to choose for that good. Tools from individual decision theory can help modelling consumer's choice behavior, in order to identify the criteria that the good has to meet.

There are more examples that underscore the importance and the wide applicability of individual decision theory. This monograph incorporates several models that contribute to this discipline in various ways. The stream that I follow in the thesis starts with the classical theories on expected utility and ends with developments of a more modern 
decision making model, namely, curnulative prospect theory. In this introductory chapter I explain the underlying structure for individual decision under uncertainty and under risk. Once these are established, in the following chapters we continue with models that describe individual decision making more specifically.

Individual decision theory distinguishes between models for uncertainty and models for risk. The basic concepts of decision under uncertainty are the set of states, the outcomes, and the acts. The set of states is the complete collection of fully described situations that can happen in the future. Exactly one such situation will happen, which is called the true state. The decision maker, however, is not sure about which will be the true state, and cannot enforce any state. This fact explains the notion of uncertainty.

An outcome is something that can affect the decision maker directly. It can be money, a commodity bundle, a health situation, etc. Outcomes are always associated with states, and the mechanism that assigns to each state a possible outcome is an act. The decision maker is faced with choices between acts. When an act is chosen and future reveals the true state, then the decision maker receives the outcome that is associated with the true state by the chosen act. It is therefore important to make a well-considered choice. Decision theory assumes that the decision maker has a preference over the acts.

A clarifying example is the following: Suppose Ms. B, the decision maker, visits a horse race, and she plans to bet some money in the next race. At the betting office she receives the information that five horses will participate in that race. The names of the horses are $h_{1}, h_{2}, h_{3}, h_{4}$, and $h_{5}$. She decides to invest money only on one bet. There are two types of tickets for sale, both at the price of $\$ 7.5$. The first one offers $\$ 5$ if horse $h_{1}$ is the winner, $\$ 10$ if horse $h_{2}$ wins, and $\$ 0, \$ 15$, and $\$ 8$, respectively, if horse $h_{3}, h_{4}, h_{5}$ wins. The second ticket offers $\$ 10, \$ 6, \$ 12, \$ 7$, and $\$ 3$ for horses $h_{1}, h_{2}, h_{3}, h_{4}$, and $h_{5}$, respectively. Ms. B carefully analyzes the situation and realizes that the collection of horses $\left\{h_{1}, h_{2}, h_{3}, h_{4}, h_{5}\right\}$ is the set of states. Exactly one horse will win the race, indicating the true state, and she is uncertain about which will be the winner. The amounts of money indicated by each ticket minus the price which is paid for the ticket are the outcomes. The resulting acts, that reveal for each horse the amount of money that will be gained if that horse wins the race, negative amounts meaning losses, are $f=(-2.5,2.5,-7.5,7.5,0.5)$ for the first ticket and $g=(2.5,-1.5,4.5,-0.5,-4.5)$ for the second ticket. Her experience motivates Ms. B to choose for the first ticket, which indicates her preference between the acts $f$ and $g$.

The setup for decision under risk is somewhat different. A set of prizes is given, and a lottery decides which prize the decision maker receives. In a lottery with each prize a 
known chance of winning that prize is associated. On the entire collection of lotteries the decision maker has a preference.

Consider, for example, a roulette wheel with three equal-sized fields, two colored red and one blue. Prizes are the amounts of money that one can gain from a single spin of this fair roulette wheel. Suppose, against a payment of $\$ 2.5$ we can choose from the following two possibilities. First, if the wheel stops at a field that is red one gets $\$ 3$, and $\$ 0$ if the final field is blue. Second, if the final field is blue one gets $\$ 5$, and $\$ 0$ if it is red. The above possibilities are the following lotteries: the first lottery gives a $2 / 3$ chance of gaining $\$ 0.5$ and a $1 / 3$ chance of losing $\$ 2.5$. The second lottery gives a $2 / 3$ chance of losing $\$ 2.5$ and a $1 / 3$ chance of gaining $\$ 2.5$. The probabilities result from the fact that two of the fields are red, the other is blue, and all fields are equal-sized, and the prizes in the lotteries are net prizes.

The preceding examples illustrate the differences between decision under uncertainty and decision under risk. For instance, in Anscombe \& Aumann (1963) an act is referred to as "horse lottery", and a lottery -as introduced above- is referred to as "roulette lottery." In this thesis, however, for decision under uncertainty, I use the setup with states, outcomes, and acts similar to Savage (1954). For decision under risk we use the setup with prizes and lotteries. To link risk and uncertainty, note that decision under risk can be considered the special case of decision under uncertainty when objective probabilities for the states are given beforehand.

We now discuss the goals to be optimized in decision situations. In health care it is assumed that physicians strive to achieve the best health situation for a patient. An important criterion is the quantity of life, that the patient can gain from a medical intervention. Also, the quality of life of the patient is central and has to be at its highest possible level. In economics, for a firm that produces certain goods, it is assumed that profit has to be maximized. This indicates that the goal of a decision process is to reach an optimal choice under the given circumstances. There are mathematical tools available that enable to deal with optimization problems. However, the application of these tools to a decision process requires in addition to the mathematical formulation of the decision problem, as introduced above, the identification of a numerical function that captures all properties of the preference relation. Such a function we call representing function or representation for the preference relation. Often, certain properties, such as continuity, concavity, monotonicity or differentiability, are associated with a representing function. It is the aim of decision science to identify characteristics in terms of preferences such that representability by an 
appropriate function is sustained. In turn, the function should be representing only for preferences with those special characteristics. Such results are compressed in a decision model.

The classical model for decision under risk is expected utility. In this model lotteries are evaluated in the following way. To each prize a utility value is assigned, which in turn is multiplied by the objective probability of receiving that prize in a given lottery. The summation over all prizes, of the utilities multiplied by their probabilities, is the expected utility of the lottery. Comparison of lotteries results in comparison of expected utility of those lotteries. The decision maker prefers a first lottery to a second one, if the first lottery has a higher expected utility value than the second one.

For decision under uncertainty the classical approach is subjective expected utihty. Under this model - assuming here that the set of states is finite - an act is evaluated as follows. A utility value is assigned to each outcome, and to each state a subjective probability indicates how likely the decision maker views that state to occur. For a given act the utility of outcomes is multiplied by the probability of the state, and the summation, over all states gives the subjective expected utility of the act. Similar to decision under risk, a higher subjective expected utility value indicates a preference for the corresponding act.

Both approaches above were considered to be rational decision making models. The reason is that the preference axioms that are used to characterize (subjective) expected utility are acceptable on their own and most people agree with them. However, the fact that many people violate expected utility in their decisions, has led to severe criticisms on the axioms. Experiments were conducted to undermine the expected utility theories, and new models that are stable against those paradoxes had to be developed.

One conclusion from the experiments that indicate violation of expected utility was the evidence that individuals tend to underestimate and/or overestimate probabilities. For decision under risk rank-dependent utility is a model that is robust against the expected utility paradoxes. For the evaluation of a lottery the rank-ordering of prizes is essential. First, the prizes are ordered from best to worst, and a utility value is assigned to each prize. Then, a probability transformation function associates decision weights (i.e. new probabilities) to each prize, depending on the original probabilities and the ordering of prizes. Finally, the summation over all prizes, of the utilities multiplied by their decision weights, is the rank-dependent utility of the lottery.

For decision under uncertainty Choquet-expected utility is the analog to rank dependent 
utility. In this model the outcomes of an act are rank-ordered, implying a ranking of the states. A capacity (i.e. a nonadditive probability measure on the set of states) determines decision weights for each state, depending on the new ranking. The summation of the utility value of an outcome multiplied by the decision weight of the state according to the outcomc, gives the Choquet-expected utility of the act.

Cumulative prospect theory generalizes rank-dependent utility (and similarly Choquetexpected utility) in permitting a different treatment for gains than for losses. It is therefore the sign-dependent generalization of RDU (CEU). The prize that has utility equal to zero is called the status quo. It separates gains (i.e. prizes preferred to the status quo) from losses (i.e. prizes dispreferred to the status quo). A lottery, then, can be decomposed into a gain-part and a loss-part. The gain-part is defined as the original lottery with all losses replaced by the status quo. Similarly, the loss-part is the original lottery with all gains replaced by the status quo. The cumulative prospect theory value of a lottery can be viewed as the sum of two rank-dependent utility values, the first measuring the loss-part and the second one measuring the gain part. The probability transformation for gains can be different from the one for losses, and the shape of utility can reveal loss aversion.

This thesis contributes to classical as well as to modern developments on individual decision making. In Chapter 2 the classical foundations of expected utility provided by Ramsey (1931), de Finetti (1931, 1937), von Neumann \& Morgenstern (1944), Anscombe \& Aumann (1963), and others are considered. The aim is to provide a unifying idea of the mentioned models. These models have in common that a cardinal utility index for outcomes (prizes), independent of the states and probabilities, can be derived. In the second chapter it is shown that this property of utility can be considered to lie at the basis of most expected utility models.

Chapter 3 presents a model that extends Savage's (1954) subjective expected utility to state dependent expected utility, and jointly generalizes the Debreu (1960)/Gorman (1968) characterization of additive decomposable functionals and separability to infinite dimensions. The representing functional for the preference is defined sirnilarly to the Lebesgue-integral. However, in the absence of an underlying probability measure, the functional extends integrals in a natural way. The results are applied to risk attitude analysis and to Bayesian updating of new information.

Chapters 4 and 5 focus on Cumulative Prospect 'Theory. Risk properties in terms of preferences are incorporated to derive models with a specific form of utility. In Chapter 4 , for decision under risk, a preference characterization with power utility is given. It is 
demonstrated that in the presence of weak ordering, continuity and stochastic dominance, constant proportional risk aversion together with comonotonic independence are sufficient for a preference characterization by a cumulative prospect theory functional. Consequently, the mentioned preference conditions inherit all that is necessary for the separation of decision weights and utility, and sign-dependence.

Then, in Chapter 5 the findings from Chapter 4 are extended for decision under uncertainty. In addition to cumulative prospect theory models with power utility, constant absolute risk aversion is considered implying linear or exponential utility. Also models with additive or multiplicative utility and multilinear utility are formulated for multiattribute decision making.

In Chapter 6, techniques from cooperative game theory are applied to multiattribute utility, resulting in characterizations of Shapley (1953)-type values for multi-choice games There is given a finite set of attributes, each of which can be present on a finite number of levels. A health state is described by indicating for each attribute exictly one level. A situation assigns to each possible health state a utility value. In Chapter 6 we provide a mechanism that measures the contributions of each level for reaching the perfect health state, i.e. the state where each attribute has maximal level. Such mechanisms can reveal important insights in health care, where the identification of criteria that determine quality of life is a central issue.

All chapters are written in article form and therefore are self-containing, so that some notation and definitions are repeated. However, this layout has the advantage that the reader can start this thesis at any preferred chapter. Proofs are usually placed in the appendix of that chapter.

The results incorporated in this thesis were first formulated in the following research papers: Chapter 2 refers to Wakker \& Zank (1998a), Chapter 3 to Wakker \& Zank (1999), Chapter 4 to Wakker \& Zank (1998b), Chapter 5 to Zank (1998), and Chapter 6 refers to Peters \& Zank (1999). 


\section{Chapter 2}

\section{A Unified Derivation of Classical Subjective Expected Utility Models through Cardinal Utility}

\subsection{Introduction}

A characteristic property of expected utility is the separation of probabilities, describing the uncertainty of a decision maker regarding a state space, and utilities, describing the value of outcomes. This separation is reflected in existing preference axiomatizations, that can be classified into two groups accordingly. First, there are the likelihood-oriented axiomatizations, in which the central property of subjective expected utility is probabilistic sophistication (i.e., uncertainty is expressed in terms of probabilities). A rich structure is imposed on the relevant uncertainties and preference axioms are based on that structure. This approach can be used in decision under risk, where probabilities are already given (von Neumann \& Morgenstern, 1944). Also Savage's (1954) approach, which does not assume probabilities given, is likelihood oriented. His postulate P4 allows the derivation of probabilities. Utilities are then derived similarly to von Neumann \& Morgenstern (1944).

The second group of axiomatizations is utility-oriented and is the subject of this chapter. Here a rich structure is imposed on the outcome set and preference axioms are based on that structure. The central property of subjective expected utility now is cardinality of utility, i.e., a meaningful ordering of utility differences (Vickrey, 1945). The approaches of

\footnotetext{
${ }^{9} T$ The results in this chapter were first. formulated in Wakker \& Zank (1998a)
} 
Ramsey (1931), de Finetti (1931, 1937), and Anscombe \& Aumann (1963) can be classified in this group. In this chapter it will be shown that all these models can be derived from one unifying principle, ensuring the existence of cardinal utility, invariant across different states of nature or context. Probability then results from the utility-exchange rate between different states.

In a formal manner, the unifying principle can also be introduced in von Neurnann \& Morgenstern's (1944) likelihood-oriented axiomatization. A corresponding derivation of their model will thus be provided. The remaining classical axiomatization, Savage's (1954), can be derived in a similar fashion if the results of qualitative probability theory (Fishburn, 1986) can be used; this idea is not elaborated here. Hence Savage's axiomatization is not covered in this chapter. Derivations of subjective expected utility (SEU) that were directly based on the unifying principle have been presented by Wakker $(1984,1989 \mathrm{~b}, 1993 \mathrm{a})$ and Wakker \& Tversky (1993). The principle was used in experimental measurements of utility by Wakker \& Deneffe (1996), Bouzit \& Gleyses (1996), Fennema \& van Assen (1997), Abdellaoui (1998), and Bleichrodt \& Pinto (1998). It can already be recognized in Krantz et al.'s (1971) "standard sequence invariance."

Nonexpected utility models can be characterized by appropriate weakenings of the unifying principle. For example, Wakker (1989a; 1989b, Chapter VI) used a "comonotonic" weakening to characterize Schmeidler's (1989) Choquet expected utility. Wakker (1994) used a similar comonotonic weakening for risk to characterize Quiggin's (1981) rankdependent utility. In these models, there is no complete separation of decision weight and utility because the decision weight of an event depends on the rank-ordering of the associated outcome. The invariant ordering of utility-differences is therefore not generally valid but only under special circumstances ("comonotonicity"). Nonexpected utility models will not be discussed in this chapter.

Section 2.2 presents the basic method for deriving expected utility from utility tradeoffs, invariant across states of nature. In subsequent sections, it is demonstrated that the utility tradeoffs can be recognized in the classical axiomatizations of SEU, i.e., de Finetti's (1937) in Section 2.3, Anscombe \& Aumann's (1963) in Section 2.4, von Neumann \& Morgenstern's (1944) in Section 2.5, and Ramsey's (1931) in Section 2.6. Section 2.7 briefly demonstrates that utility tradeoffs can also be recognized in recent SEU axiomatizations. The Appendix to this chapter presents proofs not given in the main text. For each SEU axiomatization discussed, it is first shown how the unifying principle can be recognized in the axioms used and then how the principle can serve to provide alternative derivations. 


\subsection{The Subjective Expected Utility Model}

Throughout this chapter the following notation is used. $\Gamma$ denotes the set of outcomes and $S:=\{1, \ldots, n\}, n \in \mathbb{I N}$ is a finite set of states (of nature) where exactly one state is true and the others are not true. A decision maker does not know for sure which state is the true state. Subsets of $S$ are events. An act $f$ is a mapping from $S$ to $\Gamma$, assigning the outcome $f(j)$ (or $f_{j}$ for short) to each state $j . f_{j}$ is the outcome obtained by the decision maker if he chose $f$ and the true state is $j$. For an event $E, f_{E} g$ denotes the act that assigns outcome $f_{i}$ to each state $i \in E$ and outcome $g_{j}$ to each state $j \notin E$. Similarly, for state $i$ and outcome $\alpha, \alpha_{i} f$ is ( $f$ with $f_{i}$ replaced by $\alpha$ ).

$\succcurlyeq$ denotes the preference relation of the decision maker on $\Gamma^{n}$, the set of acts. A function $V$ represents $\succcurlyeq$ if $V: \Gamma^{n} \rightarrow I R$ and $f \geqslant g \Leftrightarrow V(f) \geqslant V(g)$. If a representing function exists, then $\succcurlyeq$ is a weak order, i.e., it is complete $(f \succcurlyeq g$ or $g \succcurlyeq f$ for all $f, g$ ) and transitive. As usual, strict preference $\succ$ denotes the asymmetric part of $\succcurlyeq$ and $\sim$ the symmetric part, and $\preccurlyeq$ and $\prec$ denote reversed preferences. We call $\succcurlyeq$ weak preference. Outcomes are often identified with constant acts. Thus, the preference relation $\succcurlyeq$ generates a preference relation, also denoted by $\succcurlyeq$, over the outcomes.

Subjective expected utility (SEU) holds if there exist probabilities $p_{1}, \ldots, p_{n}$ (nonnegative and summing to one) and a utility function $U: \Gamma \rightarrow I R$ such that $f \mapsto \sum_{j=1}^{n} p_{j} U\left(f_{j}\right)$ represents $\succcurlyeq$. The utility function $U$ will be cardinal in all results in this chapter, meaning that it can be replaced by another utility function $U^{*}$ if and only if there exist real $\tau$ and positive $\sigma$ such that $U^{*}=\tau+\sigma U$.

In all main results the following monotonicity will be assumed: $f \succcurlyeq g$ whenever $f_{j} \succcurlyeq g_{j}$ for all $j$, where the preference between $f$ and $g$ is strict if the preference between $f_{j}$ and $g_{j}$ is strict for at least one $j$. This condition rules out null states, i.e., impossible states that have probability 0 . Ruling those out simplifies some subsequent definitions and notation, but is not a restriction because null events do not affect preference.

In this section, $\Gamma$ is assumed to be a convex subset of $I R^{m}$ and $\Gamma$ and $\Gamma^{n}$ are endowed with the Euclidean topology. Here the term linear is used as equivalent to the mathematical term affine, i.e., liniearity of a function need not imply that it assigns 0 to the origin. $\succcurlyeq$ is continuous if the sets $\left\{f \in \Gamma^{n} \mid f \succ g\right\}$ and $\left\{f \in \Gamma^{n} \mid f \prec g\right\}$ are open for all acts $g$. Next the preference condition is defined that captures cardinal utility and its invariance across different states, and that will be used later to derive the other expected utility models. As 
a preparation, the following notation is defined for outcomes $\alpha, \beta, \gamma, \delta \in \Gamma$ :

$$
\alpha \beta \succcurlyeq{ }^{*} \gamma \delta
$$

if there exist acts $f, g \in \Gamma^{n}$ and a state $i$ such that $\left\{\alpha_{i} f \succcurlyeq \beta_{i} g\right.$ and $\left.\gamma_{i} f \preccurlyeq \delta_{i} g\right]$. Note that, at this stage, no properties of $\succcurlyeq^{*}$ can be claimed yet. Under SEU, some can be derived from Equation (2.1) hereafter. We similarly write

$$
\alpha \beta \succ^{*} \gamma \delta
$$

if there exist acts $f, g \in \Gamma^{n}$ and a state $j$ such that $\left[\alpha_{j} f \succcurlyeq \beta_{j} g\right.$ and $\left.\gamma_{j} f \prec \delta_{j} g\right]$. Substitution of SEU elementarily shows the following implications:

$$
\begin{aligned}
& \alpha \beta \succcurlyeq{ }^{*} \gamma \delta \Rightarrow U(\alpha)-U(\beta) \geqslant U(\gamma)-U(\delta) \\
& \alpha \beta \succ^{*} \gamma \delta \Rightarrow U(\alpha)-U(\beta)>U(\gamma)-U(\delta) .
\end{aligned}
$$

Thus, the * relations capture orderings of utility differences. Now invariance of cardinal utility across states, the characteristic condition of SEU, is expressed in observable and testable terms, i.e., in terms of a preference condition. $\succcurlyeq$ satisfies tradeoff consistency if there do not exist outcomes $\alpha, \beta, \gamma, \delta$ such that $\alpha \beta \succ^{*} \gamma \delta$ and $\gamma \delta \succcurlyeq \succcurlyeq^{*} \alpha \beta$. By (2.1), it immediately follows that tradeoff consistency is a necessary condition for SEU. Wakker $(1984,1989 \mathrm{~b})$ proved that it is also sufficient in the utility-oriented approach to SEU:

THEOREM 2.1 Let $\Gamma$ be a convex subset of $\mathbb{R}^{m}$ and $n \geqslant 2$. For the binary relation $\geqslant$ on $\Gamma^{n}$ the following two statements are equivalent.

(i) SEU holds, with continuous utility and positive probabilities.

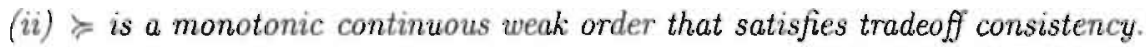

Further, utility in ( $i$ ) is cardinal and the probabilities are uniquely determined whenever $U$ is not constant.

In the notation $\alpha \beta \succcurlyeq^{*} \gamma \delta$, the state $i$ and the acts $f, g$ are suppressed. That reflects the characteristic property of $\mathrm{SEU}$, that the revelations of the $*$ relations, and the belonging utility difference orderings, have a meaning independent of states and context. Indeed, Equation (2.1) shows that the belonging ordering of utility differences always follows, independently of the specific nature of $i, f, g$. In nonexpected utility models with cardinal 
utility, such as Schmeidler's (1989) Choquet expected utility, restrictions must be imposed on revelations $\alpha \beta \succcurlyeq \succcurlyeq^{*} \gamma \delta$. For instance, in Choquet expected utility, the revelation $\alpha \beta \succcurlyeq{ }^{*} \gamma \delta$ is only valid if a "comonotonicity" restriction is verified (Wakker, 1989b, Chapter VI; Wakker \& Tversky, 1993) to preclude distortions due to "rank-dependence."

The theorem has been adapted to arbitrary (infinite) state spaces by Wakker (1993a). The purpose of the present chapter is to demonstrate as clearly as possible a common aspect of the SEU models, hence the technical complications of infinite models will not be discussed. The remainder of the chapter shows how classical derivations of SEU can be derived from Theorem 2.1 .

\section{3 de Finetti's Approach}

An early result, with the real numbers as outcome set, has been provided by de Finetti (1931, 1937). In his model, the tradeoff consistency condition for the binary relation is replaced by additivity and monotonicity with respect to the natural ordering on $\mathbb{R}$, conditions that jointly imply tradeoff consistency as will be shown in Observation 2.3. The restrictive nature of the additivity condition appears from the implied linearity of utility. Wakker's (1984) work (Theorem 2.1 in this chapter) originated as an attempt to generalize de Finetti's result to nonlinear utility.

In this section, $\Gamma=\mathbb{R}$. Strong monotonicity means monotonicity with respect to the natural ordering on $\mathbb{R}$, i.e., $f \succ g$ whenever $f_{j} \geqslant g_{j}$ for all $j$ and $f_{j}>g_{j}$ for some $j . \succcurlyeq$ is additive if $[f \succcurlyeq g] \Rightarrow[f+h \succcurlyeq g+h]$ for all acts $f, g, h \in \mathbb{R}^{n}$. Now a version of de Finetti's theorem is given.

THEOREM 2.2 The following two statements are equivalent for the binary relation $\succcurlyeq$ on $I R^{n}$.

(i) There exist positive probabilities $p_{1}, \ldots, p_{n}$ such that $f \mapsto \sum_{j=1}^{n} p_{j} f_{j}$ represents $\succcurlyeq$.

(ii) The binary relation $\geqslant$ is a strongly monotonic, additive, continuous weak order on $\mathbb{R}^{n}$.

Although de Finetti presented his result in different terms, Theorem 2.2 captures the essence. Let us briefly compare the terms used here to de Finetti's. His term "Dutch book" comes down to additivity and strong monotonicity, and his assumption that a fair 
price exists for each act comes down to completeness of preference and continuity. He did not assume the entire domain $I R^{n}$ but his results can be seen to hold on any linear subset.

The following observation shows that tradeoff consistency follows from de Finetti's conditions and is the central step in this section.

OBSERVATION 2.3 Let $\succcurlyeq$ be a strongly monotonic and additive weak order on $\mathbb{R}^{n}$. Then it satisfies tradeoff consistency.

Proof. Assume strong monotonicity, additivity, and weak ordering. For contradiction, assume a violation of tradeoff consistency. That is, there exist outcomes $\alpha, \beta, \gamma, \delta \in \mathbb{R}$ such that $\alpha \beta \succcurlyeq \succcurlyeq^{*} \delta$ and $\gamma \delta \succ^{*} \alpha \beta$. By definition this means that for a state $i$ and acts $f, g \in \mathbb{R}^{n}$ we have

$$
\alpha_{i} f \succcurlyeq \beta_{i} g \text { and } \gamma_{i} f \preccurlyeq \delta_{i} g,
$$

and for a state $j$ and acts $v, w \in \mathbb{R}^{n}$ we have

$$
\gamma_{j} v \succcurlyeq \delta_{j} w \text { and } \alpha_{j} v \prec \beta_{j} w .
$$

The preferences in (2.2) and additivity imply

$$
(\alpha-\beta)_{i}(f-g) \succcurlyeq 0 \text { and }(\gamma-\delta)_{i}(f-g) \preccurlyeq 0,
$$

which because of transitivity and monotonicity implies that $\alpha-\beta \geqslant \gamma-\delta$. The preferences in (2.3) and additivity imply

$$
(\gamma-\delta)_{j}(v-w) \succcurlyeq 0 \text { and }(\alpha-\beta)_{j}(v-w) \prec 0
$$

which because of transitivity and monotonicity implies that $\gamma-\delta>\alpha-\beta$.

Contradictory inequalities between $\alpha-\beta$ and $\gamma-\delta$ have been derived. Thus, tradeoff consistency cannot be violated.

By means of Observation 2.3, the theorem of de Finetti can be derived from Theorem 2.1. The implication (i) $\Rightarrow$ (ii) is obvious. Next, (ii) is assumed and (i) is derived. The result is trivial for $n=1$, hence $n \geqslant 2$ is assumed. All conditions of Staternent (ii) in Theorem 2.1 are satisfied, hence SEU holds for $\succcurlyeq$ with $U$ continuous and probabilities positive. $U$ is strictly increasing because of strong monotonicity. All that remains to be shown is linearity of $U$ (so that $U$ can be the identity). That follows from additivity and is elaborated in the Appendix to this chapter. 


\subsection{Anscombe \& Aumann's Approach}

Another classical result for decision under uncertainty has been provided by Anscombe \& Aumann (1963). Their results are adapted here to the notation of this chapter, and formulated in the modern version in which there is no prior mixing of acts. Differences are discussed at the end of this section. Let us note here that, whereas Anscombe and Aumann base their proof on the von Neumann-Morgenstern expected utility derivation, our proof does not invoke that derivation.

Let $X=\left\{x_{1}, \ldots, x_{m}\right\}$ be a finite set of prizes. $\Gamma$ denotes the set of lotteries, i.e., the set of all probability distributions $\left(q_{1}, x_{1} ; \ldots ; q_{m}, x_{m}\right)$ assigning probability $q_{i}$ to prize $x_{i}$ for all $i=1, \ldots, m\left(\sum_{i=1}^{m} q_{i}=1, q_{i} \geqslant 0\right.$ for all $\left.i=1, \ldots, m\right) . \quad \Gamma$ is the $m-1$ dimensional probability simplex; it is a convex subset of $\mathbb{R}^{m}$. We shall deal with linear (i.e., affine) functions $U$ from $\Gamma$ to $\mathbb{R}$. A probability distribution $\left(q_{1}, x_{1} ; \ldots ; q_{m}, x_{m}\right)$ is a convex combination $\sum_{i=1}^{m} q_{i}\left(1, x_{i}\right)$ of the degenerate probability distributions $\left(1, x_{i}\right)$ and, for linear $U$, its $U$ value is therefore $\sum_{i=1}^{m} q_{i} U\left(1, x_{i}\right)=\sum_{i=1}^{n} q_{i} u\left(x_{i}\right)$ with $u(x)=U(1, x)$ for all outcomes $x$. The latter sum can be interpreted as an expected utility form, hence linear utility can be identified with expected utility on $\Gamma$.

$S:=\{1, \ldots, n\}$ is again a finite state space and $\Gamma^{n}$ is the set of acts $f: S \rightarrow \Gamma$, $f: j \rightarrow f(j)=f_{j}$. On $\Gamma^{n}$ a binary relation, denoted by $\succcurlyeq$, is assumed. Mixing on $\Gamma^{n}$ is defined pointwise, i.e., $[\lambda f+(1-\lambda) g](s)=\lambda f(s)+(1-\lambda) g(s)$ for all $s \in S$ and $0 \leqslant \lambda \leqslant 1$.

The binary relation $\succcurlyeq$ satisfies $v N M$-independence if, for all $f, g, h \in \Gamma^{n}$ and all $0<\lambda<$ 1, we have $[f \succcurlyeq g] \Leftrightarrow[\lambda f+(1-\lambda) h \succcurlyeq \lambda g+(1-\lambda) h]$. Following Fishburn $(1970,1982)$, the continuity condition of Jensen (1967) is used: $\succcurlyeq$ is $J$-contimuous if, for all acts $f \succ g$, and $h \in \Gamma^{n}$ there exist $\lambda, \mu \in(0,1)$ such that $\lambda h+(1-\lambda) f \succ g$ and $f \succ \mu h+(1-\mu) g$. J. continuity is weaker than continuity (i.e., Euclidean continuity as defined in Section 2.2). However, in the presence of vNM-independence and monotonicity, J-continuity implies continuity for a weak order on $\Gamma^{n}$, as the next lemma shows.

LEMMA 2.4 Let $\succcurlyeq$ be a monotonic, J-continuous, and vNM-independent weak order on $\Gamma^{n}$. Then $\succcurlyeq$ is continuous on $\Gamma^{n}$.

The proof of Lemma 2.4 is given in the Appendix to this chapter.

The approach of this section is in fact a two-stage model, where in the first stage the uncertainty concerning the true state of nature is resolved and in the second stage the resulting lottery over prizes is played. A "folding-back" maximization is adopted, with 
expected utility in the second stage. In a mathernatical sense that comes down to linear utility over the outcomes. This linearity greatly simplifies mathematical derivations, hence the popularity of this approach. It has been used in many classical papers (Anscombe \&t Aumann, 1963; Pollak, 1967; Keeney \& Raiffa, 1976; Fishburn, 1970, 1982 and the references therein). Schmeidler (1989) used this approach for his famous introduction of Choquet expected utility. Numerous other papers on nonexpected utility have used the approach likewise (Drèze, 1987; Gilboa \& Schmeidler, 1989; Hazen, 1989; Klibunoff. 1995; etc.). These papers still assume folding back and expected utility (thus linear utility) in the second stage, but consider deviations from expected utility in the first. A method for further simplifying the mathematics and avoiding the complications of the two-stage approach was described by Sarin \& Wakker (1997) for Anscombe \& Aumann's (1963) SEU and by Sarin \& Wakker (1992) for Schmeidler's (1989) Choquet expected utility. The following observation is central in this section. The observation is similar to Observation 2.3 because it also derives tradeoff consistency from a linearity assumption.

OBSERVATION 2.5 Let $\succcurlyeq$ be a vNM-independent monotonic weak order on $\Gamma^{n}$. Then tradeoff consistency holds.

Proof. Assume that $\succcurlyeq$ satisfies the conditions in the Observation but assume, for contradiction, that it violates tradeoff consistency. Thus, there exist outcomes $\alpha, \beta, \gamma, \delta \in \Gamma$ such that $\alpha \beta \succcurlyeq^{*} \gamma \delta$ and $\gamma \delta \succ^{*} \alpha \beta$. By definition of the relations $\succ^{*}$ and $\succcurlyeq^{*}$, this means that for a state $i$ and acts $f, g \in \Gamma^{n}$ we have

$$
\alpha_{i} f \geqslant \beta_{i} g
$$

and

$$
\gamma_{i} f \preccurlyeq \delta_{i g},
$$

and for a state $j$ and acts $v, w \in \Gamma^{n}$ we have

$$
\gamma_{j} v \succcurlyeq \delta_{j} w
$$

and

$$
\alpha_{j} v \prec \beta_{j} w .
$$

vNM-independence and equations (2.4), (2.5), (2.6), and (2.7), respectively, imply

$$
\frac{1}{2}\left(\alpha_{i} f\right)+\frac{1}{2}\left(\delta_{i} g\right) \succcurlyeq \frac{1}{2}\left(\beta_{i} g\right)+\frac{1}{2}\left(\delta_{i} g\right) .
$$




$$
\begin{aligned}
& \frac{1}{2}\left(\beta_{i} g\right)+\frac{1}{2}\left(\gamma_{i} f\right) \preccurlyeq \frac{1}{2}\left(\beta_{i} g\right)+\frac{1}{2}\left(\delta_{i} g\right), \\
& \frac{1}{2}\left(\gamma_{j} v\right)+\frac{1}{2}\left(\beta_{j} w\right) \succcurlyeq \frac{1}{2}\left(\delta_{j} w\right)+\frac{1}{2}\left(\beta_{j} w\right) \text {, and } \\
& \frac{1}{2}\left(\delta_{j} w\right)+\frac{1}{2}\left(\alpha_{j} v\right) \prec \frac{1}{2}\left(\delta_{j} w\right)+\frac{1}{2}\left(\beta_{j} w\right) .
\end{aligned}
$$

Equations (2.8) and (2.9) and transitivity imply

$$
\frac{1}{2}\left(\alpha_{i} f\right)+\frac{1}{2}\left(\delta_{i} g\right) \succcurlyeq \frac{1}{2}\left(\beta_{i} g\right)+\frac{1}{2}\left(\gamma_{i} f\right)
$$

and equations (2.10) and (2.11) and transitivity imply

$$
\frac{1}{2}\left(\gamma_{j} v\right)+\frac{1}{2}\left(\beta_{j} w\right) \succ \frac{1}{2}\left(\delta_{j} w\right)+\frac{1}{2}\left(\alpha_{j} v\right) .
$$

Equation (2.12) implies, because of monotonicity, that $\frac{1}{2} \alpha+\frac{1}{2} \delta \geqslant \frac{1}{2} \beta+\frac{1}{2} \gamma$, equation (2.13) implies that $\frac{1}{2} \alpha+\frac{1}{2} \delta<\frac{1}{2} \beta+\frac{1}{2} \gamma$, i.e., a contradiction results. Thus, $\succcurlyeq$ cannot violate tradeoff consistency.

Next, the Anscombe \& Aumann (1963) representation theorem is derived. Actually, Anscombe \& Aumann considered general, possibly infinite, prize sets $X$ and assumed that $\Gamma$ was the set of simple (finitely supported) lotteries on $X$. Here it is assumed that $X$ is finite for simplicity of the presentation. The extension of the following theorem to general sets $X$ is easily obtained and is presented in Proposition 2.14 in the Appendix to this chapter. Another deviation is that Anscombe \& Aumann assume that also lotteries over acts are available. Their Assumption 2 guarantees that lotteries over acts can be captured by lotteries over prizes, i.e., that their setup can be reduced to the setup used here.

THEOREM 2.6 Let $\Gamma$ be the set of probability distributions over a finite set of prizes and let $n \geqslant 2$. The following two statements are equivalent for the binary relation $\succcurlyeq$ on $\Gamma^{n}$ :

(i) SEU holds, with utility $U$ linear (i.e., $U$ is an expected utility functional) and all probabilities positive.

(ii) The binary relation $\succcurlyeq$ is a weak order, it satisfies monotonicity, vNM-independence, and J-continuity.

Proof. Obviously, Statement (i) implies Statement (ii), therefore Statement (ii) is assumed and Statement (i) is derived. By Lemma 2.4 and Observation 2.5, Statement (ii) in Theorem 2.1 is implied by Statement (ii) in Theorem 2.6. Hence, there exists an SEU representation with continuous utility $U$. Linearity of $U$ is derived in the Appendix to this chapter. 


\section{5 von Neumann \& Morgenstern's Approach}

The third approach, analyzed here, is the representation theorem of von Neumann \& Morgenstern (1944). In their SEU model, probabilities are given in advance and only utilities are derived from the preference relation. The von Neumann \& Morgenstern representation theorem can be considered the version of Theorem 2.6 for $n=1$. The proof of Theorem 2.6 cannot be invoked for $n=1$, hence the case is treated separately. The case $n=1$ can be derived as a corollary from the case $n=2$ and that is the approach which is given here. Thus, whereas Anscombe and Aumann derived their result from the von NeumannMorgenstern result, here the order is reversed and the von Neumann-Morgenstern result is derived from Anscombe and Aumann's.

THEOREM 2.7 Let $\succcurlyeq$ be a binary relation on the set $\Gamma$ of simple probability distributions over a (possibly infinite) set $X$ of prizes. The follouing two statements are equivalent:

(i) $\succcurlyeq$ can be represented by expected utility (i.e., a linear $U$ ).

(ii) $\succcurlyeq$ is a weak order that satisfies $v N M$-independence and J-continuity.

Proof. The implication (i) $\Rightarrow$ (ii) is elementary, hence (ii) is assumed and (i) is derived. Theorem 2.6 and its extension to general prize sets $X$, i.e., Proposition 2.14 in the Appendix to this chapter, are used to deduce Statement (i). Let $\bar{\Gamma}$ be defined as follows: $\tilde{\Gamma}:=\left\{\left(f_{1}, f_{2}\right) \mid f_{1}, f_{2} \in \Gamma\right\}$.

On $\bar{\Gamma}$ the binary relation $\succcurlyeq$ is defined for $\bar{f}, \bar{g} \in \bar{\Gamma}$ as:

$$
\bar{f} \succcurlyeq \bar{g} \Leftrightarrow \frac{1}{2} f_{1}+\frac{1}{2} f_{2} \succcurlyeq \frac{1}{2} g_{1}+\frac{1}{2} g_{2},
$$

where $\bar{f}:=\left(f_{1}, f_{2}\right)$ and $\bar{g}:=\left(g_{1}, g_{2}\right)$ for $f_{1}, f_{2}, g_{1}, g_{2} \in \Gamma$ are interpreted as acts in a twostates Anscombe \& Aumann model with $\bar{\Gamma}=\Gamma^{2}$. It follows from elementary substitution that $\succcurlyeq$ inherits all properties from $\succcurlyeq$ described in Statement (ii) of Theorem 2.7. In addition, monotonicity of $\succcurlyeq$ follows from vNM-independence. (The following four preferences are all equivalent: $(f, f) \succcurlyeq(g, g) ; f \succcurlyeq g ; \frac{1}{2} f+\frac{1}{2} h \succcurlyeq \frac{1}{2} g+\frac{1}{2} h ;(f, h) \succcurlyeq(g, h)$; similarly, $(h, f) \succcurlyeq(h, g)$ can be derived. The equivalences for weak preferences immediately imply the equivalences for strict preferences.)

Therefore, the assumptions in Statement (ii) of Theorem 2.6 are satisfied and SEU holds for outcome set $\bar{\Gamma}$, preference relation $\bar{\zeta}$, probabilities $p_{1}, p_{2}$ and linear utility $\bar{U}$ (invoke Proposition 2.14 if $X$ is infinite). The expected utility model in Statement (i) follows from restriction to the constant acts in $\bar{\Gamma}$. It can, but need not, be seen that $p_{1}=\frac{1}{2}=p_{2}$. 


\subsection{Ramsey's Approach: The Equiprobable State Case}

An appealing axiomatization of subjective expected utility is possible if all states are equally likely. In that case, essentially, Savage's sure-thing principle alone characterizes subjective expected utility. Because the result is an almost trivial corollary of additive representation theorems, it has not received much attention in the literature. It has been used as a tool in more complex results by Blackorby, Davidson, \& Donaldson (1977) and Chew \& Epstein (1989). Let us now present the result.

$\succcurlyeq$ satisfies the sure-thing principle if $c_{i} f \succcurlyeq c_{i} g$ implies $c_{i}^{\prime} f \geqslant c_{i}^{\prime} g$ for all states $i$, "common" outcomes $c, c^{\prime}$, and acts $f, g$. That is, the preference between two acts is independent of a common outcome (e.g., c), hence remains invariant if that common outcome is replaced by another common outcome (such as $c^{\prime}$ ). Then preference is obviously independent. of any number of common outcomes, by repeated application of the principle. The surething principle was used by Debreu (1960) and Krantz et al. (1971) to characterize additive representations. That is, as soon as there are three or more states, then under the usual conditions (weak ordering, continuity, monotonicity) the sure thing principle is necessary and sufficient for additive representability through $\sum_{j=1}^{n} V_{j}\left(f_{j}\right)$. That representation is reduced to the equally-likely subjective expected utility representation $\sum_{j=1}^{n} \frac{1}{n} U\left(f_{j}\right)$ by the following exchangeability condition: $f \sim g$ whenever $g$ is obtained from $f$ by permuting: the outcomes. Let us state the result formally.

THEOREM 2.8 Let $\succcurlyeq$ be a binary relation on $\Gamma^{n}$, with $n \geqslant 3$ and $\Gamma$ a convex subset of $I R^{m}$. The following two statements are equivalent.

(i) There exists a continuous utility $U: \Gamma \rightarrow I R$ such that $\succcurlyeq$ is represented by $\sum_{j=1}^{n} \frac{1}{n} U\left(f_{j}\right)$

(ii) $\succcurlyeq$ is a monotonic continuous weak order that satisfies the sure-thing principle and exchangeability.

Utility in (i) is cardinal.

The derivation of Theorem 2.8 from existing additive representation theorems is elementary, hence no separate proof is needed. Nevertheless, next it is demonstrated informally how exchangeability and the sure-thing principle imply a version of tradeoff consistency. 
Lemma 2.9 is derived from preference conditions without invoking the additive representation. Following Lemma 2.9, a proof of Theorem 2.8 is suggested. The purpose of this analysis is to demonstrate once more the unity of expected utility models, comprising cardinal utility that is invariant across all states.

LemMA 2.9 Let, for $n \geqslant 3$, be a weak order on $\Gamma^{n}$ that satisfies the sure-thing principle and exchangeability. Then the three preferences $\left(x, \alpha, c_{3}, \ldots, c_{n}\right) \preccurlyeq\left(y, \beta, c_{3}, \ldots, c_{n}\right)$, $\left(x, \gamma, c_{3}, \ldots, c_{n}\right) \succcurlyeq\left(y, \delta, c_{3}, \ldots, c_{n}\right)$, and $\left(\alpha, v, c_{3}, \ldots, c_{n}\right) \succcurlyeq\left(\beta, w, c_{3}, \ldots, c_{n}\right)$ imply the fourth preference $\left(\gamma, v, c_{3}, \ldots, c_{n}\right) \succcurlyeq\left(\delta, w, c_{3}, \ldots, c_{n}\right)$.

Proof. The outcomes for states $4, \ldots, n$ are fixed and are suppressed. The sure-thing principle and exchangeability are used throughout the proof without explicit mentioning. The first antecedent preference in the lemma implies $(x, \alpha, \delta) \preccurlyeq(y, \beta, \delta)$, the second implies $(y, \beta, \delta) \preccurlyeq(x, \beta, \gamma)$. Because of transitivity, $(x, \alpha, \delta) \preccurlyeq(x, \beta, \gamma)$, hence $(\beta, v, \gamma) \succcurlyeq(\alpha, v, \delta)$. The third antecedent preference implies $(\alpha, v, \delta) \succcurlyeq(\beta, w, \delta)$. Transitivity now implies $(\beta, v, \gamma) \succcurlyeq(\beta, w, \delta)$. From this, finally, the consequent preference in the lemma follows.

Let us sketch, informally, how Lemma 2.9 can be used to prove Theorem 2.8. First, the sure-thing principle in Theorem 2.8 implies an additive representation $\sum_{j=1}^{n} V_{j}\left(x_{j}\right)$. Lemma 2.9 iruplies that $V_{1}$ and $V_{2}$ order utility differences the same way. Due to exchangeability, $V_{1}$ and $V_{j}$ order utility differences the same way for all $j$. That implies an expected utility representation $\sum_{j=1}^{n} p_{j} U\left(x_{j}\right)$. Exchangeability implies that all probabilities $p_{j}$ are $\frac{1}{n_{k}}$.

Next the case of $n=2$ with equally likely states 1 and 2 is treated. The preceding analysis essentially used $n \geqslant 3$, not only for invoking the additive representation but also in its derivation of tradeoff consistency in Lemma 2.9. The case $n=2$ is of historical interest because it underlies Ramsey's (1931) expected utility derivation, with 1 and 2 the "ethically neutral" events. The way of deriving a strength of preference relation $\geqslant * *$, used by Ramsey and many after him (e.g., d'Aspremont \& Gevers, 1990, see Section 2.7), is through

$$
\alpha \beta \succcurlyeq{ }^{* *} \gamma \delta \text { if }(\alpha, \delta) \succcurlyeq(\beta, \gamma) \text {. }
$$

This method requires equal likelihood of the two states. It implies $\alpha \beta \geqslant{ }^{*} \gamma \delta$ because of $(\alpha, \delta) \succcurlyeq(\beta, \gamma)$ and $(\gamma, \delta) \preccurlyeq(\delta, \gamma)$ (implied by equal likelihood and hence exchangeability). Weak ordering of $\succcurlyeq * *$, studied in the next lemma, was used by d'Aspremont \& Gevers (1990). 
LEMMA 2.10 Let $\succcurlyeq$ be an exchangeable weak order on $\Gamma^{2}$. If $\succcurlyeq * *$, defined in (2.14), is a weak order on $\Gamma^{2}$, then it agrees with $\succcurlyeq *$ and tradeoff consistency holds.

Proof. Because of exchangeability, $\succ^{* *}$, defined by $\alpha \beta^{\prime} \succ^{* *} \gamma \delta$ if $(\alpha, \delta) \succ(\beta, \gamma)$, is the asymmetric part of $\succcurlyeq *$ (not $(\gamma, \beta) \succcurlyeq(\delta, \alpha)$ implies not $\gamma \delta \succcurlyeq * \alpha \beta$ ).

Assume $\alpha \beta \succcurlyeq \succcurlyeq^{*} \gamma \delta$, say $(\mu, \alpha) \succcurlyeq(\nu, \beta)$ and $(\mu, \gamma) \preccurlyeq(\nu, \delta)$. Then, using $(\alpha, \mu) \succcurlyeq(\beta, \nu)$, we have $\alpha \beta \succcurlyeq^{* *} \nu \mu \succcurlyeq * \gamma \delta$. Transitivity of $\succcurlyeq * *$ implies $\alpha \beta \succcurlyeq{ }^{* *} \gamma \delta$. We already saw above the lemma that $\alpha \beta \succcurlyeq^{* *} \gamma \delta$ implies $\alpha \beta \succcurlyeq^{*} \gamma \delta$. Hence $\succcurlyeq *$ and $\succcurlyeq^{* *}$ are identical.

Similarly, $\alpha \beta \succ^{*} \gamma \delta$ implies $\alpha \beta \succ^{* *} \gamma \delta . \alpha \beta \succcurlyeq^{*} \gamma \delta$ and $\gamma \delta \succ^{*} \alpha \beta$ would now imply $\alpha \beta \succcurlyeq * * \gamma \delta$ and $\gamma \delta \succ^{* *} \alpha \beta$, contradicting weak ordering of $\succcurlyeq^{* *}$. Hence tradeoff corisistency must hold.

COROLLARY 2.11 Let $\succcurlyeq$ be a binary relation on $\Gamma^{2}$, where $\Gamma$ is a convex subset of $\mathbb{R}^{m}$. The following two statements are equivalent.

(i) There exists a continuous utility function $U$ such that $(\alpha, \beta) \mapsto \frac{1}{2} U(\alpha)+\frac{1}{2} U(\beta)$ represents preference.

(ii) $\succcurlyeq$ is an exchangeable continuous weak order and $\succcurlyeq * *$ is also a weak order.

Proof. (i) $\Rightarrow$ (ii) is elementary. Now assume Statement (ii). If both states are null then $U$ is constant and the corollary follows. If one state is nonnull then so is, by exchangeability, the other, and that is what we assume henceforth. According to Lemma 2.10, (ii) implies tradeoff consistency, hence subjective expected utility (monotonicity in Theorem 2.1 is only needed to imply positive probabilities and it is not needed for the subjective expected utility representation). Because of exchangeability, the states are equally-likely and have probability $\frac{1}{2}$.

\subsection{Modern SEU Derivations}

This section briefly discusses some recent derivations of SEU. First, a "bisymmetry axiom" is discussed for two states; that is, $n=2$ is assurned for now. Acts are sornetimes denoted as pairs $(x, \alpha)$, etc. We assume $\Gamma=\mathbb{R}$ and continuity and strong monotonicity. Then for each act $f$ there exists a unique certainty equivalent $C E(f)$, i.e., an outcome equivalent to $f$. Bisymmetry holds if

$$
C E(C E(x, \alpha), C E(\beta, y))=C E(C E(x, \beta), C E(\alpha, y)) .
$$


Substitution of SEU shows that the condition is necessary, because both the left- and right-hand sides have utility

$$
p_{1}^{2} U(x)+p_{1} p_{2} U(\alpha)+p_{1} p_{2} U(\beta)+p_{2}^{2} U(y) .
$$

Pfanzagl (1968) and Krantz et al. (1971, Theorem 6.10) show that bisymmetry, together with some other axioms, implies SEU. The following lemma shows how the bisymmetry axiom implies invariant cardinal utility across different states.

Lemma 2.12 Let $\Gamma=\mathbb{R}$ and $n=2$. Assume that $\succcurlyeq$ is a continuous strongly monotonic weak order. Assume $(x, \alpha) \preccurlyeq(y, \beta),(x, \gamma) \succcurlyeq(y, \delta)$, and $(\alpha, v) \succcurlyeq(\beta, w)$. Then bisymmetry implies that $(\gamma, v) \succcurlyeq(\delta, w)$.

Proof. $C E(x, \gamma) \geqslant C E(y, \delta)$ and $C E(\alpha, v) \geqslant C E(\beta, w)$ imply, because of monotonicity, that $C E(C E(x, \gamma), C E(\alpha, v)) \geqslant C E(C E(y, \delta), C E(\beta, w))$. Applying bisymmetry to both sides of the inequality yields $C E(C E(x, \alpha), C E(\gamma, v)) \geqslant C E(C E(y, \beta), C E(\delta, w))$. Because $C E(x, \alpha) \leqslant C E(y, \beta)$, monotonicity implies $C E(\gamma, v) \geqslant C E(\delta, w)$, i.e., $(\gamma, v) \succcurlyeq$ $(\delta, w)$.

The lemma shows an alternative way for deriving SEU from bisymmetry. First, an additive representation is derived (see, e.g., Krantz et al., 1971, Section 6.10.1). Next, Lemma 2.12 shows that utility differences are ordered the same way across different states. That implies SEU.

Many variations of the bisymmetry condition, and extensions to more than two states, have been presented in the literature. In such cases Lemma 2.12 can be applied to each two-dimensional subspace to show that additive value functions in an additive representation are linearly related. An example is Münnich, Maksa, \& Mokken (1997). Often, "comonotonic" restrictions of such axioms have been used to characterize rankdependent generalizations of SEU (Quiggin, 1981, Axiom 4 and Quiggin \& Wakker, 1994, Axiom 4; Chew, 1989, "weak commutativity"; Nakamura, 1990, 1992, 1995, "weak multisymmetry"). Its most appealing interpretation refers to multi-stage resolutions of uncertainty (Luce, 1988, Equations 22 and 23; Segal, 1993a, "order indifference"; Luce, 1998, "event commutativity"). An appealing variation on bisymmetry was used by Gul (1992, Assumption 2) and Chew \& Karni (1994, "act independence"). A complete logical analysis of these specific axioms and their relations to our axioms is beyond the scope of this analysis. 
Finally, d'Aspremont \& Gevers (1990) is discussed. They use two equally-likely states 1,2 to derive subjective expected utility. That is, they assume that $(\alpha, \beta, z, \ldots, z) \sim$ $(\beta, \alpha, z, \ldots, z)$ for all outcomes $\alpha, \beta, z$. They derive a strength of preference relation $\succcurlyeq * *$ similarly as in (2.14), i.e., $\alpha \beta \succcurlyeq * * \delta$ if $(\alpha, \delta, z, \ldots, z) \succcurlyeq(\beta, \gamma, z, \ldots, z)$ for some outcome $z$. They introduce a new preference condition, difference-scale neutrality, a condition that is equivalent to constant absolute risk aversion in the special case of real outcomes with linear utility: Let $f, g, f^{\prime}, g^{\prime}$ be four acts. Then $f \succcurlyeq g \Leftrightarrow f^{\prime} \succcurlyeq g^{\prime}$ whenever, for some state $t, f(s) f(t) \sim^{* *} f^{\prime}(s) f^{\prime}(t)$ and $g(s) f(t) \sim^{* *} g^{\prime}(s) f^{\prime}(t)$ for all states $s$. A detailed analysis of the logical relations between their condition and tradeoff consistency would take too much space, therefore only an illustrating example is presented.

EXAMPLE 2.13

Assume a preference relation $\succcurlyeq$ on $\mathbb{R}^{n}$, weak ordering, continuity, strong monotonicity, the sure-thing principle, equally likely states 1 and 2, difference-scale neutrality, and $n=3$. Assume

$$
(z, x, \alpha) \succcurlyeq(z, y, \beta)
$$

and

$$
(z, x, \gamma) \preccurlyeq(z, y, \delta),
$$

hence $\alpha \beta \succcurlyeq{ }^{*} \gamma \delta$. Assume that $z^{\prime}, x^{\prime}, y^{\prime}, \beta^{\prime}$ can be found such that

$$
\alpha \gamma \sim * * z z^{\prime} \sim * * x x^{\prime} \sim * * y y^{\prime} \sim * * \beta \beta^{\prime} .
$$

We show that $\alpha \beta \succcurlyeq^{* *} \gamma \delta$.

By Debreu (1960), there exists an additive representation $\sum_{j=1}^{n} V_{j}\left(x_{j}\right)$. Due to equallikelihood of 1 and $2, V_{1}=V_{2}$ may be assumed. By substitution in the definition of $\succcurlyeq^{* *}$, it follows that differences of $\left(V_{1}=\right) V_{2}$ represent $\succcurlyeq * *$. Because of difference-scale neutrality, (2.15) together with (2.17) imply $\left(z^{\prime}, x^{\prime}, \gamma\right) \succcurlyeq\left(z^{\prime}, y^{\prime}, \beta^{\prime}\right)$. As (2.17) implies $V_{2}\left(x^{\prime}\right)-V_{2}\left(y^{\prime}\right)=V_{2}(x)-V_{2}(y)$, the additive representation implies $(z, x, \gamma) \succcurlyeq\left(z, y, \beta^{\prime}\right)$. That, (2.16), transitivity, and strong monotonicity imply $\beta^{\prime} \leqslant \delta$. By $(2.17), \alpha \gamma \sim \cdots$ $\beta \beta^{\prime} \succcurlyeq * \beta \delta$, i.e., $\alpha \beta \succcurlyeq{ }^{* *} \gamma \delta$. A similar reasoning with strict preference in (2.16) shows that $\alpha \beta \succ^{*} \gamma \delta$ implies $\alpha \beta \succ^{* *} \gamma \delta$.

These reasonings show how $\succcurlyeq *$ and $\succ^{*}$ revelations for state 3 correspond to $\succcurlyeq *$ and $\succ^{* *}$, hence to $V_{1}$ differences when (2.17) can be satisfied; (2.17) can always be satisfied "locally." Therefore, such $\succcurlyeq *$ and $\succ$ * revelations cannot contain inconsistencies, and utility 
differences for state 3 must be the same as for the first two states, first locally, then as a consequence also globally. In other words, the additive representation is a subjective expected utility representation.

For more than three states, similar reasonings can be applied to any state $j$ for $j \geqslant 3$, by keeping the outcomes for other states than $1,2, j$ fixed.

\subsection{Appendix: Proofs to Chapter 2}

Deriving linearity of utility in Theorem 2.2: Assume Statement (ii) and hence, as derived in the text, assume SEU with continuous utility $U$ and positive probabilities. It is proved here that additivity of $\succcurlyeq$ implies linearity of $U$.

Consider an indifference $\alpha_{1} f \sim \beta_{1} g$. Adding up $(\varepsilon, 0, \ldots, 0)$ for $\varepsilon \in \mathbb{R}$ implies

$$
\left(\alpha+\varepsilon, f_{2}, \ldots, f_{n}\right) \sim\left(\beta+\varepsilon, g_{2}, \ldots, g_{n}\right)
$$

because of additivity. Substitution of SEU in both indifferences implies

$$
U(\alpha+\varepsilon)-U(\beta+\varepsilon)=U(\alpha)-U(\beta)
$$

This holds for all $\alpha, \beta$ for which $f$ and $g$ can be found such that $\alpha_{I} f \sim \beta_{1} g$. That is, for each $z \in \mathbb{R}$ an open neighborhood can be found in which the equality (2.18) holds. This implies local linearity of $U$, thus global linearity. $U$ can be taken equal to the identity.

Proof OF LemMa 2.4. In order to prove that $\succcurlyeq$ is continuous on $\Gamma^{n}$ it has to be shown that the sets

$$
\left\{g \in \Gamma^{n} \mid g \prec h\right\} \text { and }\left\{g \in \Gamma^{n} \mid g \succ h\right\}
$$

are open in $\Gamma^{n}$ for each $h \in \Gamma^{n}$.

The set of prizes $X=\left\{x_{1}, \ldots, x_{m}\right\}$ is finite. We prove openness of $A:=\left\{g \in \Gamma^{n} \mid g \prec h\right\}$. Take any $g \in A$, hence $g \prec h$. Because the set of prizes is finite, there exists a "best" prize $y \in X$, i.e., $y \geqslant x$ for each prize $x$. Because of J-continuity, there exists a $0<\mu<1$ such that $\mu y+(1-\mu) g \prec h$. Because of monotonicity, $y \succcurlyeq f$ for every act $f$. By repeated application of vNM-independence, for each $0 \leqslant \mu^{\prime} \leqslant \mu$ we have $h \succ \mu y+(1-\mu) g \succcurlyeq$ $\mu^{\prime} y+\left(1-\mu^{\prime}\right) g \succcurlyeq \mu^{\prime} f+\left(1-\mu^{\prime}\right) g$. Hence, if $g \in A$, then we have an open neighborhood $\left\{f^{\prime} \in \Gamma^{n}: f^{\prime}=\mu^{\prime} f+\left(1-\mu^{\prime}\right) g\right.$ for some $0 \leqslant \mu^{\prime}<\mu$ and $\left.f \in \Gamma^{n}\right\}$ of $g$ in $A$. This implies that $A$ is an open subset of $\Gamma^{n}$. Similarly, $\left\{g \in \Gamma^{n} \mid g \succ h\right\}$ is an open subset of $\Gamma^{n}$. $\succcurlyeq$ is 
continuous.

DERIVING LINEARITY OF UTILITY IN THEOREM 2.6: First the following implication is derived:

$$
\alpha_{1} f \sim\left(\frac{\alpha+\beta}{2}\right)_{1} g \Rightarrow\left(\frac{\alpha+\beta}{2}\right)_{1} f \sim \beta_{1} g .
$$

Assume, for contradiction, that

$$
\alpha_{1} f \sim\left(\frac{\alpha+\beta}{2}\right)_{1} g \text { and } \beta_{1} g \succ\left(\frac{\alpha+\beta}{2}\right)_{1} f .
$$

Taking $\frac{1}{2} / \frac{1}{2}$ mixtures of the left-hand sides and of the right-hand sides we get, by repeated application of vNM-independence and transitivity,

$$
\left(\frac{\alpha+\beta}{2}\right)_{1} \frac{f+g}{2} \succ\left(\frac{\alpha+\beta}{2}\right)_{1} \frac{f+g}{2},
$$

contradicting reflexivity. Similarly, (2.20) with $\prec$ instead of $\succ$ also implies a contradiction of reflexivity. Hence (2.19) must hold true.

(2.19) implies, for $z=\frac{\alpha+\beta}{2}, \alpha z \succcurlyeq * z \beta$ and $\alpha z \preccurlyeq \preccurlyeq^{*} z \beta$ hence, by (2.1),

$$
U(\alpha)-U\left(\frac{\alpha+\beta}{2}\right)=U\left(\frac{\alpha+\beta}{2}\right)-U(\beta)
$$

which is equivalent to

$$
U\left(\frac{\alpha+\beta}{2}\right)=\frac{1}{2}[U(\alpha)+U(\beta)] .
$$

Because of continuity of $U$ in the SEU model, for each $\gamma$ in the convex set $\Gamma$ there exists an open neighborhood in which, for each $\alpha$ and $\beta, f$ and $g$ can be found to imply the first. indifference in (2.19). (2.21) follows in the open neighborhood of $\gamma$, hence the continuous $U$ is linear there. If it is locally linear, then it is globally linear.

Proposition 2.14 Theorem 2.6 can be modified by letting $X$ be infinite and $\Gamma$ the set of all simple probability distributions over $X$.

Proof. If all prizes are equivalent then, because of monotonicity, the theorem is trivial; $U$ then is constant. Next suppose there are two prizes $\alpha, \beta$ such that $\alpha \succ \beta$. Set $U(\beta)=$ $0, U(\alpha)=1$. Consider any finite subset $Y$ of $X$ containing $\alpha$ and $\beta$. If we restrict attention to prizes from $Y$, then Theorem $2.6 \mathrm{can}$ be invoked, giving an SEU model with probabilities $p_{j}^{Y}$ and utility $U^{Y}$. We may set $U^{Y}(\beta)=0$ and $U^{Y}(\alpha)=1$. For another finite subset $Z$ 
of $X$ containing $\alpha$ and $\beta$, we get an SEU model with probabilities $p_{j}^{Z}$ and utility $U^{Z}$ with, again, $U^{Z}(\beta)=0$ and $U^{Z}(\alpha)=1$. Now consider the finite set $Y \cup Z$. Because of uniqueness, the two SEU models coincide when attention is restricted to prizes from $Y \cap Z$. That is, any two such SEU models coincide on overlapping domain. Therefore the superscripts $Y, Z$ can be dropped and probabilities $p_{j}$ and utility $U$ result such that SEU represents preferences when restricted to any finite subset of prizes. That suffices to describe all preferences between acts involving only simple lotteries over prizes. 


\section{Chapter 3}

\section{State Dependent Expected Utility for Savage's State Space}

\subsection{Introduction}

Separability is one of the most important tools available for simplifying complex optimization, where several criteria have to be aggregated into one overall goal. Under some technical conditions, separability amounts to additive decomposability (Debreu, 1960; Gorman, 1968), and it has been used in many areas. Examples are decision under uncertainty (Savage, 1954), consumer theory (Barten \& Böhm, 1982), interpersonal aggregation (Fleming, 1952; Harsanyi, 1955; Broome, 1991), dynamic optimization (Strotz, 1956; Kooprnans, 1972), and many other areas (Krantz et al., 1971; Keeney \& Raiffa, 1976). Debreu's theorem, as well as its many variations, only consider finitely many criteria. In many areas, the restriction to finitely many criteria is undesirable. It is often desirable to deal with infinitely many states of nature in decision under uncertainty, infinitely many persons in group aggregation, etc.

Whereas most preference representations in the literature are routinely extended from finite to infinite dimensions, no such extension has as yet been established for Debreu's result. Providing such an extension, for the special case of real-valued outcomes and monotonic preferences, is the purpose of this chapter. The development of this new functional, additively decomposable on infinite-dimensional spaces, is similar to the definition of integrals and is also derived from approximations through step functions from above and below.

\footnotetext{
${ }^{0}$ The results in this chapter were first formulated in Wakker \& Zank (1999)
} 
The research of this chapter started with the search for a state-dependent generalization of Savage's (1954) expected utility, and a characterization thereof entirely in terms of endogeneous preference conditions. Probability is then no longer identifiable and the result is an additively decomposable functional. The state-dependent generalization of Savage (1954) is presented in Theorem 3.11. In view of this application, the main text of this chapter is formulated for the context of decision under uncertainty. It could also have been formulated for interpersonal or intertemporal or other aggregations.

The outline of this chapter is as follows. Section 3.2 surveys related literature on statedependent expected utility. It also points out that this analysis only deals with cardinal state dependence. Ordinal state independence is preserved because utility for money is assumed increasing in every state. For economic applications, this restriction seems natural. Section 3.3 discusses the absence of a general state-dependent expected utility functional in the literature thus far. In Section 3.4, elementary results are described for finite dimensions. State-dependent utility then coincides with additive conjoint measurement. Section 3.5 describes the difficulties for infinite state spaces. In particular, Example 3.4 motivates the specific form of the functional and shows why a completely general additive functional for infinite state spaces is not adopted here. Section 3.6 describes some natural preference conditions for state-dependent expected utility. The new functional is derived from these preference conditions. Section 3.7 demonstrates that the functional can be written as an integral if a countable additivity condition is added. Section 3.8 describes applications of the new functional to updating, the characterizations of risk attitudes, and the elicitation of probability. Section 3.9 summarizes and concludes. Sections 3.10 and 3.11 present mathematical modifications, and Appendices A-E, finally, present proofs.

\subsection{State-Dependent Expected Utility}

The most famous result in decision under uncertainty was provided by Savage (1954), who presented preference conditions for subjective expected utility. Savage assumed that outcomes have a meaning and value independent of the state of nature with which they are associated. In several applications, however, it is undesirable to disentangle the value of an outcome from the associated state. An example is health insurance, in which the value of money is dependent on sickness or health. Other examples are described by Karni (1985) and Drèze (1987, Chapter 8). Therefore, several papers have dealt with state-dependent generalizations of Savage's model, where the utility of an outcome is permitted to depend 
on the state of nature with which it is associated.

A central issue in state-dependent expected utility is the nonidentifiability of probability. If utility can depend on the state of nature in any way, then probabilities are no longer uniquely determined. For identifying probability, most papers in the literature add exogeneous assumptions, invoking information other than observable choice. Examples are influence of the decision maker on probabilities and states and "idempotent" acts (Drèze, 1961; 1987 Chapter 2), preferences between acts conditioned on different events (Luce \& Krantz, 1971; Fishburn, 1973), hypothetical probabilities of states set by an experimentor (Karni, Schmeidler, \& Vind, 1983), lotteries with known probabilities over state-dependent outcomes belonging to different states (LaValle \& Fishburn, 1991, Section 5), preferences and utilities conditional on null states (Rubin, 1949, 1987), and availability of some stateindependent outcomes (Karni, 1993a, 1993b).

This chapter focuses on a stage prior to the identifiability of probability. A general state-dependent extension of Savage's (1954) expected utility form is presented that is based solely on choice making and exhibits the characteristic inseparability of probability and utility. The aim is therefore not to "resolve" the inseparability of probability and utility, but rather to accept it and incorporate it into a decision-model. Such an inseparability has been recommended by Kreps (1988, Formula 7.13), and appears in Fishburn (1970, Theorem 13.1), Rubin (1987), and Nau (1995). It has, however, not yet been presented for Savage's infinite-state model. It must be kept in mind that, without a given probability measure, no integration operation is available to define the functional form. A result on state-dependent utility for infinite state spaces that does not invoke an integral is Rubin (1987). (A similar result for finite state spaces is Theorem 13.1 in Fishburn, 1970.)

The paper in the literature closest to this work is Grodal \& Mertens (1976), where probability and utility are also inseparable. They assume that an underlying countably additive measure on the state space is given a priori, with respect to which an absolute continuity condition is imposed. Thus, their functional can still be written as an integral form, contrary to Theorem 3.11 below. In Theorem 3.12 below, their result is extended by deriving the countably additive measure endogeneously, i.e., entirely from preference.

To be precise here, note that in this analysis also an additional restriction is assumed. It concems, however, the most common and relevant special case for economic science: Monetary outcomes are assumed and monotonicity and continuity. These conditions are entirely defined in terms of preferences. They imply state independence in an "ordinal" sense, i.e., outcomes are ordered the same way for different states of nature. Therefore, 
the analysis of this chapter only addresses state dependence of utility in a cardinal sense. Example 3.4 describes a case of ordinal state dependence and indicates some of its unwarranted implications which further motivate the restriction to ordinal state independence.

There is another reason for the absence of a general state-dependent extension of Savage's expected utility in the literature. Many studies have restricted attention to linear utility. Whereas linearity sometimes refers to riskless outcomes themselves (Chateauneuf, 1991; Nau, 1995), it mostly refers to probabilistic mixing (Drèze, 1961, 1987; Fishburn, 1970, Theorem 13.1; Fishburn, 1973; Karni, Schmeidler, \& Vind, 1983; Karni, 1985, 1993a; Rubin, 1987; LaValle \& Fishburn, 1991), in line with Anscombe \& Aumann (1963). Under linear utility, the distinction between ordinal and cardinal state independence disappears: a linear function is an ordinal (increasing) transform of another linear function, if and only if it is a cardinal (linear) transform. Hence, if two linear state-dependent utility functions are ordinally equivalent, they are also cardinally equivalent. This explains how Anscombe \& Aumann (1963) could obtain state-independence by only imposing an ordinal state-independence axiom (Assumption 1 there).

In Savage's model, there is no linear utility and P3 implies only ordinal, not cardinal, state independence. As pointed out by Karni (1993b, p. 433), it is primarily P4 (likelihood ordering; the only axiom violated in Theorem 3.11) which implies cardinal state independence. A partial weakening of Savage's P4, leading to a partial degree of state dependence, is studied by Karni \& Schmeidler (1993). Wakker (1987) adapted Karni, Schmeidler, \& Vind's approach (1983) to continuous, as opposed to linear, utility. In his model, ordinal and cardinal state independence were distinguished.

In a mathematical sense, the analysis here is related to Chew \& Wakker (1996) and uses similar tools. There, an "outcome-dependent" capacity (nonadditive measure) was introduced that generalizes existing rank-dependent nonexpected utility theories by dropping the separation of utility and nonadditive probability. Similar rank-dependent forms appeared in Green \& Jullien (1988), Quiggin (1989), and Segal (1993b) for decision under risk.

An alternative extension of additive conjoint measurement to infinite product sets was studied by Vind (1990). His functional shares with the functional presented here the additivity property. It need not satisfy pointwise monotonicity and thus need not be constructable from limits of simple acts; this is illustrated in Example 3.4. His assumptions on domain are more restrictive than those considered here. For details see Section 3.10, Observation $3.15(\mathrm{~g})$ and the subsequent discussion. 
The main characterizing preference condition in this model is the sure-thing principle. Other than that, only common preference axioms (weak ordering, monotonicity, continuity) are used. The results of this chapter can, therefore, be considered a counterpart to Machina \& Schmeidler (1992): They studied Savage's model in which, of the two critical expected-utility axioms, P4 (ensuring likelihood ordering) was kept and P2 (the surething principle) was dropped. Under a strenghtening of P4, they then obtained a model for "probabilistic sophistication" that followed Savage in expressing uncertainties in terms of probabilities but, due to the dropping of $\mathrm{P} 2$, did not order probability distributions according to expected utility. Machina \& Schmeidler's result was generalized by Epstein \& Le Breton (1993), who used a weaker strengthening of Savage's P4 than Machina \& Schmeidler in order to obtain an appealing interpretation as dynamic consistency. As compared to these works, here Savage's P2 is maintained and P4 is dropped. In other words, the existence of subjective probabilities and a likelihood ordering is abandoned.

The preceding duality has a remarkable implication for updating, elaborated in Section 3.8. Machina \& Schmeidler (1992) preserve the probability calculus of Bayesian updating in a nonexpected utility framework where updated preferences then depend on what would have happened outside the conditioning event. In this model, updating also results as a natural generalization of the traditional updating of expected utility, but the only point where it deviates is in the abandoning of prior probability. It preserves independence of updated preferences from counterfactual events, which is a foundation of the likelihood principle (Poirier, 1988). According to the likelihood principle, the optimal estimate of an unknown parameter or the optimal decision to reject or accept a hypothesis is, given an observed value of a statistic, independent of what one would have done given other values of the statistic. The likelihood principle is central in Bayesian statistics and is preserved in the present approach. In summary, the result presented here suggests a central role for the sure-thing principle and a lesser role for prior probabilities in the normative debate on Bayesian updating. Prior probabilities simplify Bayesian updating but are not essential.

\subsection{Absence of General State-Dependent Expected Utility in the Literature}

To explain the absence, hitherto, of a general state-dependent extension of Savage's model, assume first, contrary to Savage's model, that the state space is finite. In this case, general state-dependent expected utility is well-understood. Assume that the state space $S$ is 
$\left\{s_{1}, \ldots, s_{n}\right\}$. Let $f$ be an act, assigning outcome $f\left(s_{j}\right) \in \mathbb{R}$ to each state $s_{j}$. The expected utility formula of $f$ is given in Table 1, Formula (a1),

\begin{tabular}{|c|l|lr|}
\hline & finite dimensions & general (infinite) dimensions \\
\hline Expected utility & $\sum_{j=1}^{n} p_{j} U\left(f\left(s_{j}\right)\right)(\mathrm{a} 1)$ & $\int_{S} U(f(s)) d P$ & (b1) \\
\hline State-dependent expected utility & $\sum_{j=1}^{n} p_{j} U_{j}\left(f\left(s_{j}\right)\right)(\mathrm{a} 2)$ & $\int_{S} U_{s}(f(s)) d P$ & (b2) \\
\hline Additive decomposability & $\sum_{j=1}^{n} V_{j}\left(f\left(s_{j}\right)\right)(\mathrm{a} 3)$ & $?$ & (b3) \\
\hline
\end{tabular}

TABLE 1. Existing and non-existing functionals

where $p_{j}$ is the probability of state $s_{j}$ and $U$ denotes utility. A first version of the statedependent generalization is given in Formula (a2), where the subscript in $U_{j}$ indicates state-dependence. Any alternative state-dependent representation

$$
\sum_{j=1}^{n} q_{j} W_{i}\left(f\left(s_{j}\right)\right)
$$

with $p_{j} U_{j}=q_{j} W_{j}$ for all $j$ represents the same preferences and thus is empirically indistinguishable from the original representation. Hence only the products $p_{j} U_{j}$ are meaningful and it is preferable to rewrite the functional as in Formula (a3) where $V_{j}=p_{j} U_{j}$ for all $j$. This summation provides the state-dependent generalization of expected utility. As pointed out by Kreps (1988, Formula 7.13), state-dependent expected utility therefore is a special case of "additive conjoint measurement," axiomatized by Debreu (1960), Gorman (1968), Krantz, Luce, Suppes, \& Tversky (1971), and others. The factoring out of probability $p_{j}$ and utility $U_{j}$ as in (a2) has little significance, i.e., probability is not identifiable under state-dependent expected utility. This point has often been discussed (Drèze, 1961, 1987; Karni, 1985, 1993a, 1993b, 1996; Rubin, 1987; Kadane \& Winkler, 1988; Schervish, Seidenfeld, \& Kadane, 1990; Karni \& Schmeidler, 1993; Nau, 1995). An empirical method for eliciting the product of probability and state-dependent utility has been described by Wakker \& Deneffe (1996, end of Section 4).

Statedependent utility is more complicated for infinite state spaces. For these spaces, expected utility reads as in (b1) in the table, where $P$ denotes the probability distribution over $S$. State-dependent utility at first reads as (b2). Again, the same basic indeterminacy of probability and utility exists as in the finite state case, and alternative representations

$$
\int_{S} W_{s}(f(s)) d Q
$$


can be chosen that represent the same preferences. For instance, let $Q$ have any positive density with respect to $P$, then divide $U_{s}$ by that density to obtain $W_{s}$. As for finite state spaces, one would like to drop the meaningless factorization into $P$ and $U_{s}$. However, a functional to drop the factorization from (b2) is not readily available in the literature, hence the question mark at (b3) in the table. Here, the analogy with finite state spaces stops.

Introducing the extension of an additively decomposable functional for infinitely many states, i.e., filling in Formula (b3), is the first task. The extension is not very difficult given ordinal state independence, and is defined by enclosure from above and below by simple acts, completely analogous to the definition of integrals. The absence in the literature of the required functional, and the desirability of developing it, was pointed out by Hübner \& Suck (1993, p. 631 and Concluding remarks). For related comments restricted to finite state spaces, see Fishburn (1970, Chapter 12).

\subsection{Definitions and Preliminary Results}

$S$ is a state space that can be finite or infinite. It is endowed with an algebra $\mathcal{A}$ of subsets, that is, $\mathcal{A}$ contains the universal event $S$, the complement $A^{c}$ relative to $S$ of each of its elements $A$, and, finally, the union $A \cup B$ of each pair of elements $A, B$. Subsets of $S$ contained in $\mathcal{A}$ are called events. For any partition of $S$ or of any subevent of $S$, it is always assumed without further mentioning that its elements are events. The outcome space is $\mathbb{R}$. (Connected topological outcome spaces are discussed in Section 3.11.) An act is a bounded function from $S$ to the outcome space that is measurable, i.e., the inverse of every interval is an event. $\mathcal{F}$ denotes the set of all acts. Note that, without further mentioning, acts will be assumed to be bounded throughout this chapter. A technique for dealing with unbounded acts is described by Wakker (1993a).

For act $f$ and event $A, f_{A}$ denotes the restriction of $f$ to $A$. For acts $f, g$, and event $A, f_{A} g$ (abbreviating $f_{A} g_{A^{c}}$ ) denotes the act that agrees with $f$ on $A$ and with $g$ on $A^{c}$. Constant acts are sometimes identified with their associated outcomes. We may thus write, for outcome $x, f_{A} x$ for the act that agrees with act $f$ on event $A$ and is constant $x$ on $A^{c}$; the notation $x_{A} f$ is similar. For event $A, 1_{A}$ denotes the indicator function of $A$. For a finite partition $\left\{A_{1}, \ldots, A_{n}\right\}$ of $S, \sum_{j=1}^{n} x_{j} 1_{A_{j}}$ denotes the act assigning $x_{j}$ to each $s \in A_{j}$, for each $j$. Such an act, taking only finitely many values, is a simple act. $\mathcal{F}^{s}$ denotes the set of all simple acts. 
The preference relation is a binary relation $\succcurlyeq$ on $\mathcal{F}$. It is a weak order if it is complete $(f \succcurlyeq g$ or $g \succcurlyeq f$ for all acts $f, g)$ and transitive. The notation $\succ, \sim, \preccurlyeq$, and $\prec$ is standard, i.e., $f \succ g$ if $f \succcurlyeq g$ and not $g \succcurlyeq f, f \sim g$ if $f \succcurlyeq g$ and $g \succcurlyeq f, f \preccurlyeq g$ if $g \succcurlyeq f$, and $f \prec g$ if $g \succ f$. If there exists a representing function $V$ (i.e., $V$ is a real-valued function on the set of acts $\mathcal{F}$ such that $f \succcurlyeq g \Leftrightarrow V(f) \geqslant V(g)$ for all acts $f, g)$, then $\succcurlyeq$ is necessarily a weak order. Let us now summarize the structural assumptions made in the main body of this paper.

Assumption 3.1 (Structural Assumption). Acts are bounded measurable maps from the state space $S$ to the outcome space IR. The preference relation $\succcurlyeq$ is a binary relation on the set $\mathcal{F}$ of acts.

Simple acts play an important role in this analysis and several intermediate results will be formulated for simple acts. We will also see that several preference conditions can be restricted to simple acts. Together with some technical conditions, they then imply the corresponding conditions for the nonsimple acts. Event $A$ is null if $f_{A} g \sim g$ for all simple acts $f$ and $g$. Strict monotonicity holds if $x_{A} f \succ y_{A} f$ for all nonnull events $A$, simple acts $f$, and outcomes $x>y$.

The main condition of interest in this analysis is Savage's (1954) sure-thing principle. It requires

$$
c_{I} f \succcurlyeq c_{l} g \Leftrightarrow c_{l}^{\prime} f \succcurlyeq c_{l}^{\prime} g
$$

for all acts $c, c^{\prime}, f, g$, and events $I$. The sure-thing principle holds on $\mathcal{F}^{s}$ if the condition is only imposed on all simple acts $c, c^{\prime}, f, g$. The condition is known under various names, such as (strong) separability in consumer choice theory (Barten \& Böhm, 1982), preferential independence in multiattribute utility theory (Keeney \& Raiffa, 1976), and (con)joint independence in conjoint measurement theory (Krantz et al., 1971).

If $S$ is finite, say $S=\left\{s_{1}, \ldots, s_{n}\right\}$, then $\mathcal{F}$ can be identified with $I R^{n}$ through the bijective mapping $f \rightarrow\left(f\left(s_{1}\right), \ldots, f\left(s_{n}\right)\right)$. Continuity of $\succcurlyeq$ then means that, for each act $f,\{g: g \succcurlyeq f\}$ and $\{g: g \preccurlyeq f\}$ are closed subsets of $\mathbb{Z} R^{n}$ with respect to the Euclidean topology. Debreu (1960) proved the following result, formulated here for the context of decision under uncertainty.

Theorem 3.2. (Debreu, 1960; state-dependent expected utility for finite state spaces). Let the Structural Assumption 3.1 hold and let $S=\left\{s_{1}, \ldots, s_{n}\right\}$, where at least three states are nonnull. Then the following two statements are equivalent: 
(i) There exist continuous functions $V_{j}: \mathbb{R} \rightarrow \mathbb{R}, j=1, \ldots, n$, that are strictly increasing for all nonnull states and constant for all null states, and such that $\succcurlyeq$ is represented by

$$
V(f)=\sum_{j=1}^{n} V_{j}\left(f\left(s_{j}\right)\right) .
$$

(ii) $\succcurlyeq$ is a continuous strictly monotonic weak order that satisfies the sure-thing principle.

The following uniqueness holds for (i): $W(f)=\sum_{j=1}^{n} W_{j}\left(f\left(s_{j}\right)\right)$ represents $\succcurlyeq$ if and only if there exist real numbers $\tau_{1}, \ldots, \tau_{n}$ and a positive $\sigma$ such that $W_{j}=\tau_{j}+\sigma V_{j}$ for all $j$, implying that $W=\tau+\sigma V$ for $\tau=\tau_{1}+\cdots+\tau_{n}$.

Strictly speaking, the uniqueness result in Theorem 3.2 may be considered slightly stronger than Debreu's (1960) because continuity of $W$ has not been presupposed but instead follows as a consequence (Wakker, 1988). This generalized uniqueness result is used in what follows. The case of exactly one nonnull state is trivial; then there is no uncertainty. For two states of nature, an additional condition must be added in Statement (ii), for instance, the "hexagon condition" or the "Thomsen condition" (Karni \& Safra, 1998).

To obtain a (state-independent) expected utility representation in Statement (i), and thus a finite-state version of Savage's (1954) result, the conditions in Statement (ii) must be strengthened. Such strengthenings have been provided by Grodal (1978), Wakker (1984, 1989b), Nakamura (1990), and Gul (1992). An essential intuitive step in attaining such a separation of probability and utility is an identification, in at least a cardinal sense, of outcomes contingent on different states of nature. For further discussion and a clarifying example of this point, see Karni (1996).

\subsection{Complications for Infinite State Spaces}

Following Savage (1954), now a nonatomicity condition is introduced, that implies infinity of $S$. An event $A$ is an atom if, for every subevent $B$ of $A$, either $B$ or $A \backslash B$ is null. It is assumed that $S$ contains no atoms. This assumption is somewhat weaker than the "atomless" condition (see Section 3.10) commonly adopted in the literature, but suffices for the purposes of this analysis. 
A major complication in the extension to infinite state spaces concerns the topological restrictions to be imposed. Continuity with respect to the product topology is too restrictive for the purposes of this chapter. Therefore a "finite-dimensional" simple-continuity condition is imposed on $\mathcal{F}^{s}$ which requires that, for each finite partition $\left\{A_{1}, \ldots, A_{n}\right\}$ of $S$, the preference relation over the finite-dimensional subspace of acts of the form $\sum_{j=1}^{n} x_{j} 1_{A_{j}}$ is continuous. That is, for every $\left(x_{1}, \ldots, x_{n}\right) \in \mathbb{I}^{n},\left\{\left(y_{1}, \ldots, y_{n}\right) \in \mathbb{R}^{n}: \sum_{j=1}^{n} y_{j} 1_{A_{j}} \succcurlyeq\right.$ $\left.\sum_{j=1}^{n} x_{j} 1_{A_{j}}\right\}$ and $\left\{\left(y_{1}, \ldots, y_{n}\right) \in \mathbb{I R}^{n}: \sum_{j=1}^{n} y_{j} 1_{A_{j}} \preccurlyeq \sum_{j=1}^{n} x_{j} 1_{A_{j}}\right\}$ should be closed subsets of $I R^{n}$.

Proposition 3.3 Assume that $S$ contains no atoms and that $\succcurlyeq$ is a simple-continuous strictly monotonic weak order that satisfies the sure-thing principle on $\mathcal{F}^{s}$. Then for each event $A$ there exists a continuous and either strictly increasing or constant function $V_{A}$ : $\mathbb{I R} \rightarrow \mathbb{I R}$ such that $\succcurlyeq$ is represented on $\mathcal{F}^{s}$ by

$$
V\left(\sum_{j=1}^{n} x_{j} 1_{A_{j}}\right)=\sum_{j=1}^{n_{n}} V_{A_{j}}\left(x_{j}\right) .
$$

The following uniqueness holds: $W\left(\sum_{j=1}^{n} x_{j} 1_{A_{j}}\right)=\sum_{j=1}^{n} W_{A_{j}}\left(x_{j}\right)$ represents $\succcurlyeq$ on $\mathcal{F}^{s}$ if and only if there exist real numbers $\tau_{A}$ for each event $A$ and a positive $\sigma$ such that $W_{A}=$ $\tau_{A}+\sigma V_{A}$ for all $A$, where $\tau_{A \cup B}=\tau_{A}+\tau_{B}$ for all disjoint events $A, B$ and $W=\tau_{S}+\sigma V$.

The uniqueness result, up to multiplication by a positive "scale" factor $\sigma$ and addition of a signed bounded finitely additive measure $\tau$ is a characteristic of state-dependent utility, and will return in later theorems.

Proposition 3.3 can be interpreted as a state-dependent extension of Savage's (1954) result for simple acts. Next the extension to nonsimple acts is considered. It turns out that, in the presence of weak ordering and the sure-thing principle, the technical conditions in the proposition, i.e., strict monotonicity and simple-continuity, do not suffice to ensure the desired representation. Loosely speaking, the sure-thing principle and strict monotonicity only impose restrictions within finite-dimensional subspaces of $\mathcal{F}$. Different finite-dimensional subspaces of $\mathcal{F}$ that share only the origin can be entirely unrelated. These claims are illustrated by the following example.

EXAMPLE 3.4

Assume that $S=[0,1]$, is endowed with the regular Borel $\sigma$-algebra (the smallest $\sigma$ algebra containing all intervals) and the Lebesgue measure (assigning to each interval its 
length). The functional $V$ that represents $\succcurlyeq$ is linear. On $\mathcal{F}^{s}, V$ is expected value. First a description is given of the extension of $V$ from $\mathcal{F}^{s}$ to some, but not all, nonsimple acts, for three different versions of the example. Then it is explained how $V$ can be extended to all nonsimple acts for each of the three versions. Finally, the example is discussed.

(VERSION A) For $f(s)=s+1, V(f)=-1$.

(VERsion B) Consider the partition $\pi=\{[0,1 / n),[1 / n, 2 / n), \ldots,[1-1 / n, 1),[1]\}$. Let $\mathcal{F}^{*}$ contain all acts that are 0 at all points $j / n$ and are linear on each interval of the partition. $\mathcal{F}^{*}$ is a linear space that intersects $\mathcal{F}^{s}$ only at the origin (the constant 0 act). Let $P^{*}$ be any arbitrary probability measure on $[0,1]$ and $U^{*}$ any continuous function from $\mathbb{R}$ to $\mathbb{R}$ such that $U^{*}(0)=0 ; U^{*}$ need not be increasing. Let $V$ be expected utility with respect to $P^{*}$ and $U^{*}$ on $\mathcal{F}^{*}$.

(VERSion C) Let $\mathcal{F}^{*}$ and $\mathcal{F}^{* *}$ be two linear subspaces of $\mathcal{F}$ such that the linear space spanned by any two of $\mathcal{F}^{s}, \mathcal{F}^{*}$ and $\mathcal{F}^{* *}$ intersects the third only at the origin. Let $P^{*}$ and $P^{* *}$ be arbitrary probability measures on $[0,1]$ and $U^{*}$ and $U^{* *}$ any continuous functions from $\mathbb{R}$ to $\mathbb{R}$ such that $U^{*}(0)=0=U^{* *}(0)$. Let $V$ be expected utility with respect to $P^{*}$ and $U^{*}$ on $\mathcal{F}^{*}$, and expected utility with respect to $P^{* *}$ and $U^{* *}$ on $\mathcal{F}^{* *}$.

In each of the three versions we can extend $V$ to a linear functional on all of $\mathcal{F}$ by the Hahn-Banach extension theorem (Dunford \& Schwartz, 1958). Linearity of $V$ implies the sure-thing principle because

$$
\begin{aligned}
V\left(c_{I} f\right)-V\left(c_{I} g\right) & =V\left(c_{I} 0\right)+V\left(0_{I} f\right)-\left(V\left(c_{I} 0\right)+V\left(0_{I} g\right)\right)=V\left(0_{I} f\right)-V\left(0_{I} g\right) \\
& =V\left(c_{I}^{\prime} 0\right)+V\left(0_{I} f\right)-\left(V\left(c_{I}^{\prime} 0\right)+V\left(0_{I} g\right)\right)=V\left(c_{I}^{\prime} f\right)-V\left(c_{I}^{\prime} g\right) .
\end{aligned}
$$

Therefore, $V\left(c_{I} f\right)-V\left(c_{I} g\right)$ and $V\left(c_{I}^{\prime} f\right)-V\left(c_{I}^{\prime} g\right)$ have the same sign and the preference between $c_{I} f$ and $c_{I} g$ is the same as between $c_{I}^{\prime} f$ and $c_{I}^{\prime} g$. Because $V\left(x_{A} f\right)-V\left(y_{A} f\right)=$ $(x-y) V\left(1_{A}\right)>0$ for $x>y$ and $A$ nonnull, strict monotonicity also holds for the nonsimple acts.

A phenomenon occurs in (A) that is unwarranted from a decision theoretical viewpoint: $f(s)>0$ for all states $s$, but still $V(f)<0$ and $f$ is strictly less preferred than 0 . Another unwarranted phenomenon occurs in both (B) and (C): the restriction of $V$ (and, correspondingly, of $\succcurlyeq$ ) to several subparts of its domain are completely unrelated. All three cases (A), (B), and (C) illustrate that $V$ on $\mathcal{F}^{s}$ does not restrict $V$ to other parts of its domain to a satisfactory degree. 
A natural condition for preference functionals $V$ in decision under uncertainty, violated in (A) of the example, is pointwise monotonicity: if $f(s) \geqslant g(s)$ for all $s$, then $V(f) \geqslant V(g)$. Similarly, pointwise monotonicity for $\succcurlyeq$ requires that $f \succcurlyeq g$ if $f(s) \geqslant g(s)$ for all $s$ (compare Rubin, 1987, Axiom 0).

Next the extension of additivity is provided. A functional $V: \mathcal{F} \rightarrow \mathbb{R}$ is additively decomposable over disjoint events, or additive for short, if for each event $A$ there exists a functional $V_{A}$ on the set of restrictions $f_{A}$ of acts $f$ to $A$ such that for each finite partition $\left\{A_{1}, \ldots, A_{n}\right\}$ of $S$,

$$
V(f)=\sum_{j=1}^{n} V_{A_{j}}\left(f_{A_{j}}\right) .
$$

It is important to note that $V_{A}\left(f_{A}\right)$ does not depend on the partitioning of $A^{c}$ and neither on the part of $f$ outside of $A$. Linear functionals are additive (define $V_{A}\left(f_{A}\right)=V\left(f_{A} 0\right)$ ). Also, the expected utility functional (Formula (b1) in Table 1) is additive (define $V_{A}=$ $\int_{A} U(f(s)) d P$ ). Additive functionals were previously studied by Vind (1990).

Additivity of $V$ immediately implies that the functionals $V_{A}$ are also additive in the sense that $V_{B \cup C}\left(f_{B \cup C}\right)=V_{B}\left(f_{B}\right)+V_{C}\left(f_{C}\right)$ for all disjoint events $B, C$. The following lemma shows that additivity on $\mathcal{F}^{s}$ is in agreement with the functional described in Proposition 3.3 (that imposes (3.3) only when the $f_{A_{j}}$ are constant).

LEMMA $3.5 V: \mathcal{F}^{s} \rightarrow \mathbb{R}$ is additive if and only if there exists, for each event $A$, a function $V_{A}^{*}: \mathbb{R} \rightarrow \mathbb{R}$ such that $V\left(\sum_{j=1}^{n} x_{j} 1_{A_{j}}\right)=\sum_{j=1}^{n} V_{A_{j}}^{*}\left(x_{j}\right)$.

Because of its importance, the following observation is displayed and its, elementary, proof is presented in the main text.

OESERVATION 3.6 If $\succcurlyeq$ can be represented by an additive functional then it satisfies the sure-thing principle.

Proof. Writing $R$ ("relevant") for the complement of event $I$ ("irrelevant"), we get

$$
V\left(c_{I} f\right)-V\left(c_{I} g\right)=V_{I}\left(c_{I}\right)+V_{R}\left(f_{R}\right)-\left(V_{I}\left(c_{I}\right)+V_{R}\left(g_{R}\right)\right)
$$

and

$$
V\left(c_{l}^{\prime} f\right)-V\left(c_{l}^{\prime} g\right)=V_{i}\left(c_{l}^{\prime}\right)+V_{R}\left(f_{R}\right)-\left(V_{I}\left(c_{l}^{\prime}\right)+V_{R}\left(g_{R}\right)\right)
$$

Dropping the terms $V_{I}\left(c_{l}\right)$ in the first equality and the terms $V_{i}\left(c_{i}^{\prime}\right)$ in the second, the same right-hand sides result. Hence the left-hand sides are also equal. Then they surely 
have the same sign, and this implies that the preference between $c_{I} f$ and $c_{I} g$ is the same as between $c_{I}^{\prime} f$ and $c_{I}^{\prime} g$.

Additivity does not preclude the phenomena described in Example 3.4 because the representing functional there was linear and thus additive. For infinite-dimensional extensions of additive conjoint measurement, there may be interest in general functionals, as in Example 3.4, that do not satisfy a pointwise monotonicity condition (Streufert, 1995). For economic applications of decision under uncertainty with monetary outcomes, however, there may be little empirical interest in such general functionals. Therefore, the next section follows a different route, avoiding the complications of Example 3.4.

\subsection{State-Dependent Expected Utility for Infinite State Spaces}

In this extension of state-dependent expected utility to all acts in $\mathcal{F}$, the empirically most important conditions of additivity and the sure-thing principle will not be extended to all of $\mathcal{F}$ from the start, but will initially only be imposed explicitly on $\mathcal{F}^{s}$. Instead, continuity will be extended to all of $\mathcal{F}$. This ensures that the functional $V$ can be extended from $\mathcal{F}^{s}$ to $\mathcal{F}$ in the same way as integrals: Each nonsimple act is enclosed between dominating and dominated simple acts that converge to that act in supnorm. Because of continuity, the value of the nonsimple act is the limit of the values of the converging simple acts. Thus, $V$ is a "natural" extension of integrals. A crucial implication of supnorm continuity, underlying this procedure, is pointwise monotonicity (Lemma 3.9). We subsequently find, as a "bonus," that additivity and the sure-thing principle, imposed only on $\mathcal{F}^{s}$, do hold on all of $\mathcal{F}$ after all. Thus, a satisfactory version of state-dependent expected utility has been obtained.

Under the supnorm, the distance between two acts $f, g$ is $\sup _{s \in S}|f(s)-g(s)| . V$ is supnorm-continuous if $\{f \in \mathcal{F}: V(f) \geqslant \lambda\}$ and $\{f \in \mathcal{F}: V(f) \leqslant \lambda\}$ are supnom-closed for all $\lambda \in \mathbb{R}$. Similarly, $\succcurlyeq$ is supnorm-continuous if, for all acts $f$, the sets $\{g \in \mathcal{F}: g \geqslant f\}$. and $\{g \in \mathcal{F}: g \preccurlyeq f\}$ are supnorm-closed. It is well-known that supnorm-continuity is equivalent to the common continuity on $I R^{n}$, and thus supnorm-continuity implies simplecontinuity. That supnorm-continuity is not overly restrictive may be further accentuated by its equivalence to continuity of utility under expected utility and also under the more general Choquet expected utility, introduced by Schmeidler (1989). This equivalence was 
demonstrated by Chew \& Wakker (1996, Observation 2); it should be kupt in mind here that the acts here are assumed to be bounded.

A number of steps in the derivation of the main result, Theorem 3.11, are made explicit in the text. These steps make it clear that the state-dependent expected utility functional proposed here generalizes integrals in a natural manner. Henceforth, up to Theorem 3.11, it is assumed that:

ASSUMPTION $3.7 S$ contains no atoms and $\succcurlyeq$ is a strictly monotonic supnorm-continuous weak order that satisfies the sure-thing principle on $\mathcal{F}^{s}$.

This assumption implies that Proposition 3.3 can be applied, yielding an additive representing functional $V$ on $\mathcal{F}^{s}$. The functional $V$ is first extended to all of $\mathcal{F}$, through "certainty equivalents." Next, the required conditions are derived for $V . \gamma \in \mathbb{R}$ is a certainty equivalent for act $f$ if $\gamma \sim f$.

LEMma 3.8 Under Assumption 3.7, there exists a certainty equivalent for each act $f$.

Because of strict monotonicity (applied to the constant acts), there exists at most one certainty equivalent for each act. We extend the functional $V$ of Proposition 3.3 to the nonsimple acts by defining $V(f)=V(\gamma)$ where $\gamma$ is the certainty equivalent of $f$. This functional obviously represents preferences and, as shown in Lemma 3.19 in Appendix A, is continuous. It obviously agrees with the definition on $\mathcal{F}^{s}$. An important observation is the following.

Lemma 3.9 Under Assumption 3.7, the functional $V$ as just defined satisfies pointwise monotonicity.

Because of this lemma, the $V$ value of a nonsimple act $f$ results from $V$ values of simple acts in a similar fashion as an integral value of $f$ would. This can be seen as follows. It. is well-known that there exist two sequences of simple acts $a^{j}$ and $b^{j}$ ( $a$ abbreviating "above" and $b$ "below") such that

$$
f(s)+1 / j \geqslant a^{j}(s) \geqslant a^{j+1}(s) \geqslant f(s) \geqslant b^{j+1}(s) \geqslant b^{j}(s) \geqslant f(s)-1 / j
$$

for all $s$ and $j$ (see Figure 3.1). Then, because of pointwise monotonicity, $V(f)$ is enclosed increasingly tightly by $V\left(b^{j}\right) \leqslant V(f) \leqslant V\left(a^{j}\right)$. Because of supnorm-continuity (Lemma 3.19), $V\left(b^{3}\right)$ and $V\left(a^{j}\right)$ indeed converge to $V(f)$. The definition of the functional is similar 


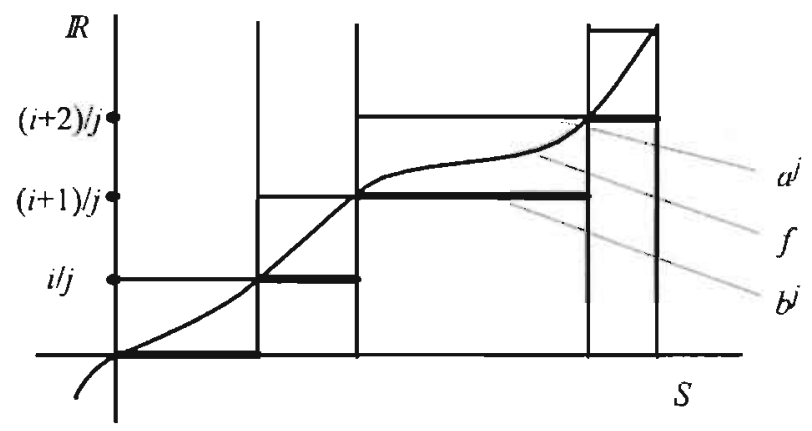

Figure 3.1: Enclosing $f$ from above and below by simple acts $a^{j}$ and $b^{j}$.

to the construction of the Lebesgue integral. It does not invoke any structure on the state space other than measure-theoretical and in this serse is more general than the Riemann integral.

It will next be demonstrated that $V$ inherits additivity on $\mathcal{F}$ from additivity' on $\mathcal{F}^{s}$. By Observation 3.6, this also ensures the sure-thing principle for all acts.

Lemma 3.10 Under Assumption 3.7, $V$ as just constructed satisfies additivity.

At this point, the main theorem of this paper can be given.

THEOREM 3.11 (State-dependent extension of Savage, 1954). Let the Structural Assumption 3.1 hold and assume that $S$ contains no atoms. Then the following two statements are equivalent:

(i) $\succcurlyeq$ is represented by a functional $V: \mathcal{F} \rightarrow \mathbb{R}$ that satisfies (1) additivity, i.e., there exist functionals $V_{A}$ defined on the restrictions of acts to $A$ such that

$$
V(f)=\sum_{j=1}^{n} V_{A_{j}}\left(f_{A_{j}}\right)
$$

for each finite partition $\left\{A_{1}, \ldots, A_{n}\right\}$ of $S$; (2) supnorm-continuity; (3) for each $A$, $V_{A}(x)$ (for outcome/constant act $x$ ) is either constant or strictly increasing in $x$. 
(ii) $\succcurlyeq$ satisfies: (1) weak ordering; (2) strict monotonicity; (3) supnorm-continuity; (4) the sure-thing principle.

Furthermore, (i) implies pointwise monotonicity of $V$ and (ii) implies pointwise monotonicity of $\succcurlyeq$. In (i), $V_{A}(x)$ is continuous in $x$ for each $A$.

The following uniqueness holds for (i): $W$ is additive $\left(W(f)=\sum_{j=1}^{n} W_{A_{j}}\left(f_{A_{j}}\right)\right.$ for any finite partition $\left\{A_{1}, \ldots, A_{n}\right\}$ of $\left.S^{\prime}\right)$ and represents $\succcurlyeq$ if and only if there exist real numbers $\tau_{A}$ for each event $A$ and a positive $\sigma$ such that $W_{A}=\tau_{A}+\sigma V_{A}$ for all $A$, where $\tau_{A \cup B}=\tau_{A}+\tau_{B}$ for all disjoint events $A, B$ and $W=\tau_{S}+\sigma V$.

The theorem provides a state-dependent extension of Savage's expected utility. It does not provide a separation of probability and (state-dependent) utility, but instead it provides a decision-model where these factors are inextricably joined together.

\subsection{Deriving an Integral Form under Countable Additivity}

This section demonstrates that the new functional can be written as an integral with respect to a countably additive measure $\mu$, under a stronger continuity condition. The result is given here for its mathematical convenience, not for its empirical content. The convenience results because the well-known integration techniques can now be invoked. The measure $\mu$ and the accompanying state dependent utility function $U_{s}$ are, however, only mathematical devices and do not represent additional empirical content. All relevant empirical information is contained in the general additively decomposable functional, without any measure $\mu$ or utility $U_{s}$ specified.

It is assumed in this section that the algebra $\mathcal{A}$ is a $\sigma$-algebra on $S$, i.e., it is closed under countable (instead of just finite) unions. Note that, in the special case of Theorem 3.11 where the functional $V$ can be decomposed into a probability measure $P$ and a utility function $U$, the axioms presented here only imply finite additivity of $P$. The following continuity condition reinforces supnorm continuity to the effect of also implying countable additivity.

$\succcurlyeq$ satisfies pointwise continuity if a sequence of "uniformly bounded acts that converge pointwise to an act also converge preference-wise to that act." A sequence of acts $f^{j}$ is uniformly bounded if there exist outcomes $x, y$ such that $x \geqslant f^{j}(s) \geqslant y$ for all $j$ and $s$, and 
it converges pointwise to $f$ if $\lim _{j \rightarrow \infty} f^{j}(s)=f(s)$ for all $s$. It converges preference-wise to $f$ if, for each act $g, g \succ f$ implies the existence of a $J \in I N$ such that $g \succ f^{j}$ for all $j>I$ and $g \prec f$ implies the existence of a $J \in \mathbb{N}$ such that $g \prec f^{j}$ for all $j>J$. Pointwise continuity of the functional $V$ is defined likewise: If a sequence of uniformly bounded acts converge pointwise, then their $V$ value also converges to the $V$ value of their limit. Pointwise continuity implies supnorm continuity (Lemma 3.20) and countable additivity (Lemma 3.22). It is weaker than (i.e., implied by) continuity of the state-dependent utility functions $U_{s}$ in (i) in the theorem (Lemma 3.27), hence is not overly restrictive.

Theorem 3.12 (State-dependent expected utility under countable additivity). Let the Structural Assumption 3.1 hold and assume that $S$ contains no atoms. Then the following two statements are equivalent:

(i) There exists a countably additive measure $\mu$ on $S$ and for each state $s$ a strictly increasing (state-dependent utility) function $U_{s}: \mathbb{R} \rightarrow I R$ such that $\succcurlyeq$ is represented by the pointwise continuous integral

$$
f \mapsto \int_{S} U_{s}(f(s)) d \mu .
$$

(ii) $\succcurlyeq$ satisfies: (1) weak ordering; (2) strict monotonicity; (3) pointwise continuity; (4) the sure-thing principle.

The following uniqueness holds for (i): $\mu,\left(U_{s}\right)_{s \in S}$ can be replaced by $\mu^{*},\left(U_{s}^{*}\right)_{s \in S}$ if and only if $\mu$ and $\mu^{*}$ have the same null events and $U_{s}^{*}=\tau(s)+\sigma \delta(s) U_{s}$ for all $s$ except on a $\mu$ null event, where $\tau$ is a measurable function from $S$ to $\mathbb{I R}, \sigma$ is a positive constant, and $\delta$ is the Radon-Nikodym density function of $\mu$ with respect to $\mu^{*}$.

The integral in (i) is well-defined and real-valued for each act $f$. It is crucial for the uniqueness result regarding $\mu$ that each state-dependent utility is required to be strictly increasing. This excludes the case in which $\mu(A)>0$ but $A$ is null because of constant utility at $A$. Hence, $\mu$ identifies null events (Lemma 3.26). Statement (i) obviously implies pointwise monotonicity of $V$, and Statement (ii) of $\succcurlyeq$, as in Theorem 3.11. Theorem 3.12 deviates from Grodal \& Mertens' (1976) result mainly because the measure $\mu$ is not given a priori but is determined endogeneously, completely in terms of preferences. 


\subsection{Applications}

This section presents some applications of Theorem 3.11. First it is demonstrated, on the basis of suggestions by Karni, Schmeidler, \& Vind (1983) and Karni (1996), that updating through adjusted likelihoods ratios, the corner stone of Bayesian statistics, is still possible. Thus, Bayesian updating is extended from expected utility to state-dependent expected utility.

As a preparation, the classical updating from Bayesian statistics, which will be generalized hereafter, is described within the expected utility model. Let $f \mapsto \int_{S} U(f(s)) d P$ represent the prior preferences over acts, for a subjective probability measure $P$ and a (subjective) utility function $U$. Assume that we observe a value $X$ of some "statistic." The probability (density) for this observation is different for different states of nature, i.e., it is a function of the state of nature. This function is the likelihood function.

To avoid some technical complications, the likelihood function is assumed to take only finitely many values and is further assumed to be constant on each element $A_{j}$ of a partition $\left\{A_{1}, \ldots, A_{n}\right\}$. In other words, the observation of $X$ helps to distinguish between the "hypotheses" $A_{1}, \ldots, A_{n}$; conditional on any $A_{j}$, however, the observation $X$ does not provide further information. Say the likelihood of the observation is $\lambda_{j}$ for each $A_{j}$. Then, after the observation the probability for any $A_{j}$ is changed by a factor proportional to $\lambda_{j}$ according to the formula of Bayes. The updated preference relation, denoted $\tilde{\xi}$, can be represented by $f \mapsto \sum_{j=1}^{n} \lambda_{j} \int_{A_{j}} U(f(s)) d P$; note here that normalization is not necessary for the representation of preferences.

The previously-discussed method for updating preference is first extended to statedependent utility when prior probabilities are known. Let the prior preferences be represented by $f \mapsto \int_{S} U_{s}(f(s)) d P$. With prior probability $P$ given, we can still apply Bayes' formula in the classical manner, and posterior preferences are represented by

$$
f \longmapsto \sum_{j=1}^{n} \lambda_{j} \int_{A,} U_{s}(f(s)) d P .
$$

This manner of updating can be inferred from Karni, Schmeidler, \& Vind (1983, Lemma); see also Karni (1996, Section 3.3), Karni \& Schmeidler (1993), and Nau (1995, Section 7). Karni, Schmeidler, \& Vind also demonstrate that an alternative equivalent state-dependent representation $f \mapsto \int_{S} W_{s}(f(s)) d Q$ for prior preference can be similarly updated into

$$
f \mapsto \sum_{j=1}^{n} \lambda_{j} \int_{A_{j}} W_{s}(f(s)) d Q .
$$


This updated form represents the same posterior preferences as the updated form in (3.4). In other words, alternative representations for state-dependent expected utility remain indistinguishable under updating. From this observation, only one further step is required to extend the updating to the new functional in which there is no factorization into probability and utility. If prior preferences are represented by an additive functional $V(f)$, then updated preferences are represented by

$$
f \mapsto \sum_{j=1}^{n} \lambda_{j} V_{A_{j}}\left(f_{A_{j}}\right) .
$$

Normalization can be obtained by letting the functional assign value 0 to the act that is constant 0 and value 1 to the act that is constant 1 . The sure-thing principle is at the heart of this updating method, and prior probabilities play no role.

The updating method presented here, is characterized for state-dependent expected utility with a subjective likelihood function. Likelihood is not objective and given a priori but is determined in terms of the decision maker's preferences. A topic for future research is under what conditions such subjective likelihoods coincide with objective likelihoods if the latter are available.

The preference relation $\succcurlyeq$ again designates "prior" preferences, relevant before the receipt of information. Posterior preferences are indicated by a wiggle. Thus, $\bar{\sim} ;, \xi$, and ₹ relate to posterior preferences and are derived from $\succcurlyeq$ as the related symbols are from $\succcurlyeq$. An observation is informative with respect to a partition $\left\{A_{1}, \ldots, A_{n}\right\}$ if, for each $j$. either $A_{j}$ is a null event for $\succcurlyeq$ ("impossible a posteriori"), or $f_{A,} c \succcurlyeq g_{A}, c$ if and only if $f_{A_{j}} c$ $\succcurlyeq g_{A j} c$, for all acts $f, g_{2} c$. This means that the information does not distinguish between subevents of any $A_{j}$. In other words, given any $A_{j}$ nothing changes. The information only concerns the mutual likelihoods ratios of the different events ("hypotheses") $A_{j}$ in the partition. A case in which the condition is satisfied is when, giver $A_{j}$, the true state of nature in $A_{j}$ is determined by a random mechanism (such as repeated coin tosses) unrelated to the observation.

THEOREM 3.13 Assume that the prior preferences are denoted by $\geqslant$ and that preferences are updated into $₹$ after some observation. Assume that both preference relations satisfy the conditions and statements of Theorem 3.11 (for the same state and act space). Then the following two statements are equivalent for a partition $\left\{A_{1}, \ldots, A_{n}\right\}$ of the state space:

(i) There exist nonnegative numbers $\lambda_{j}$ such that the representation of Theorem 3.11 holds for $\succcurlyeq$, and $f \mapsto \sum_{j=1}^{n} \lambda_{j} V_{A_{j}}\left(f_{A_{j}}\right)$ represents the updated preferences $\succcurlyeq$. 


\section{(ii) The observation is informative with respect to the partition $\left\{A_{1}, \ldots, A_{n}\right\}$.}

This theorem demonstrates that Bayesian updating is possible even if a convenient decomposition $V_{A_{j}}=P\left(A_{j}\right) U$ is not available. Prior probability, the target of much criticism, apparently is not crucial for Bayesian updating.

Next a study of risk attitudes is provided. For finite state spaces, similar result were presented by Miyamoto \& Wakker (1996). Constant risk aversion holds if $f \sim x \Leftrightarrow$ $f+\epsilon \sim x+\epsilon$, for all acts $f$, outcomes $x$, and positive $\epsilon$.

Theorem 3.14 Assume that the conditions and statements of Theorem 3.11 hold. Then constant risk aversion holds if and only if expected utility holds and $U(x)=a+b e^{c x}$ for some constants $a, b, c$ with $b c>0$, or $U(x)=a+b x$ for some constants $a, b$ with $b>0$.

Similar results can be proved for positive outcomes and proportional ("relative") risk aversion. They follow from Theorem 3.14 through the replacement of all outcomes by their logarithms. Here a conjecture is formulated: Theorem 3.14, with state-dependent utility assumed but state-independent utility implied (and, of course, more general utility functions), holds true if one weakens constant risk aversion to decreasing risk aversion. That conjecture can be proved if Wakker (1989b, Conjecture VII.6.10) is true.

Theorem 3.14 and the results subsequently suggested are negative for state-dependent expected utility because common assumptions regarding risk attitude are simply not possible under state dependence. The model necessarily reduces to expected utility. Apparently, the common conditions for risk attitude need to be redefined for state-dependent utility. Alternative definitions were proposed by Karni (1983).

Finally an idea of Karni (1993a, 1993b) for identifying probability is discussed. Assume that acts $a, b$ are given and suppose that, on the basis of prior assumptions, the statedependent utility of $b$ must be 0 and the state-dependent utility of $a$ must be 1 , for all states. Then probabilities are directly identified in Theorem 3.11 through $P(A)=\frac{V\left(a_{A} b\right)-V(b)}{V(a)-V(b)}$ for each event $A$. Note that the information on utilities of $a$ and $b$ is not derived from preference but is exogeneous. The identification of subjective probability just described is related to the method of Drèze (1987, Section 2.8.3), where moral hazard and "idempotent" acts are used to identify constant-valuation acts, Karni (1993a, 1993b, 1997), where the range (thus the highest and lowest value) of utility across different states is assumed identical, Nau \& McCardle (1991) and Nau (1995), where avoidance of arbitrage implies the existence of "risk-neutral probabilities" which are defined relative to the market currency, the method 
of Karni \& Schmeidler (1993), where the assumption is reduced to a differential form, i.e., the utility of 0 is 0 for all states and has the same derivative at 0 for all states, the method of Maher (1993, Chapter 8) where two outcomes with state-independent utility are assumed, and, finally, Klibanoff (1995), who justifies the assumption of equally-valued outcomes in a dynamic setup with opportunity sets as outcomes.

The derivation of a state-dependent utility function from the preceding assumptions is a topic for future research. It requires measure-theoretical axioms regarding the role of null events and adaptation of the Radon-Nikodym theorem to finitely additive measures, subjects that are outside the scope of this chapter.

If only two outcomes are given instead of a continuum as in this analysis, then the additively decomposable functional reduces to a finitely additive measure. For that case, many advanced results have been obtained (Fishburn 1986, 1992).

This analysis has used the framework of decision under uncertainty, in which the functional represents state-dependent expected utility. The functional can be used in other areas as well. In dynamic optimization (Strotz, 1956; Koopmans, 1972), it generalizes discounted utility by allowing utility to depend on time. In welfare theory, it permits utilitarianism where utility depends on the individual (Harsanyi, 1955), but no lotteries over income need be invoked. It thus extends Fleming (1952), an appealing but not very well-known predecessor of Harsanyi (1955) and Debreu (1960), to infinite populations.

\subsection{Conclusion}

This chapter has presented the following new results.

(1) Additive utility theory (Debreu, 1960; Krantz et al., 1971) has been extended to infinite dimension.

(2) By means of (1), a state-dependent extension of Savage's (1954) expected utility has been obtained. Additional restrictions such as assumptions outside the decision theoretical domain or a measure on the state space, are not invoked.

(3) By means of (2), it has been argued that the sure-thing principle, rather than prior probability, is at the heart of Bayesian statistics.

(0) The extension of Debreu's (1960) additive utility requires an additional innovation that should precede Step (1): The appropriate functional has to be introduced. It generalizes integration naturally, with pointwise monotonicity excluding anomalies. With this functional available, state-dependent utility can be studied without invoking additional 
structure outside the realm of decision theory.

\subsection{Extensions and Further Comments}

In this section, mathematical extensions of the presented theorems are provided.

OBSERVATION 3.15 The following additions and modifications are possible in Theorem 3.11.

(a) The sure-thing principle in (4) of Statement. (ii) need only be imposed on $\mathcal{F}^{s}$.

(b) The $V_{A} s$ in (i) are supnorm-continuous.

(c) In (i), additivity of $V$ need only be imposed on $\mathcal{F}^{s}$ and then implies additivity on all of $\mathcal{F}$.

(d) If (i) or (ii) holds, then for a null event $A, g_{A} f \sim h_{A} f$ for all acts $f, g, h$ (including the nonsimple ones).

(e) If (ii) holds, then strict monotonicity holds on all of $\mathcal{F}$, i.e., $x_{A} f \succ y_{A} f$ whenever $x>y$ and $A$ is nonnull, also if the acts are nonsimple.

(f) The nonatomicity condition can be weakened by only requiring the existence of three disjoint nonnull events.

(g) The domain of preference need not be the entire set $\mathcal{F}$ but can be any set between $\mathcal{F}$ and $\mathcal{F}^{s}$ such that, for each element $f$ and event $A$, also $f_{A} 0$ is contained.

The results in (a), (c), (d), and (e) illustrate conditions that, when only imposed on the simple acts, extend to the nonsimple acts. In particular, the result in (d) shows that the dennition of null events, which is used here and which only concerns simple acts, is equivalent to the common definitions of null events that concern all acts.

The generalization in (f) covers not only the finite case considered in Theorem 3.2 but also infinite state spaces with atoms. In the main result of this analysis the nonatomic case is chosen because it is closest to Savage's model, the model that has received most attention in the literature. In Savage's (1954) expected utility model, a somewhat stronger condition was implied, i.e., that the finitely additive probability measure should be atomless: for each event $A$ and each $0<x<P(A)$ there should exist a subevent $B$ of $A$ with $P(B)=x$. If $P$ 
is countably additive, that condition is equivalent to the nonatomicity condition assumed here.

The domain restriction described in $(\mathrm{g})$ generalizes the requirement of Grodal \& Mertens (1976) and Vind (1990) that the domain be an "independent mixture" and contain all constant acts, which in particular generates all simple acts. Their connectedness condition implies (by Vind's Theorem III.1.1) the simple-continuity and boundedness conditions used here (by Vind's Remark following Corollary V.3.4). The extension of the results presented here to nonreal outcomes is described in Section 3.11.

\subsection{Connected Topological Outcome Spaces}

This section demonstrates how the outcome set can be generalized to any connected topological space. This generalization is similar to Appendix 2 in Wakker (1994) and Appendix B in Chew \& Wakker (1996). Also Grodal \& Mertens (1976) consider outcomes more general than monetary. In their approach, the outcome set should be a separable metric space and connectedness is imposed directly on the preference topology.

$\sum_{j=1}^{n} x_{j} 1_{A}$, still denotes the simple act assigning outcome $x_{j}$ to $A_{j}$ for each $j$. The natural ordering $\geqslant$ on outcomes is replaced by the preference relation $\succcurlyeq$ restricted to the constant acts, for instance in the definitions of strict and pointwise monotonicity and in the definition of strictly increasing functions. Consequently, equalities $x=y$ are sometimes replaced by equivalences $x \sim y$. f from $S$ to the outcome set is bounded if there exist outcomes $x, y$ such that $[\forall s \in S: x \preccurlyeq f(s) \preccurlyeq y]$. This definition of bounded can be somewhat more restrictive than the common term bounded, for instance if the outcome set is ]0, 1[. Measurability now requires that the inverse of each "preference interval" is an event. A preference interval is a subset of the outcome set that contains, for each $x \preccurlyeq z$, also each element $y$ such that $x \preccurlyeq y \preccurlyeq z$. Our measurability condition is ensured, in the presence of continuity of $\succcurlyeq$, by measurability with respect to any algebra containing the topology on the outcome set. If $S$ is endowed with a $\sigma$-algebra, then it suffices that all inverses under $f$ of $\{x: x \geqslant y\}$ and $\{x: x \preccurlyeq y\}$ are events. $f$ from $S$ to the outcome set is an act if it is bounded and measurable.

Certainty equivalents need no longer be uniquely determined. Throughout the following analysis, it will never matter which of several certainty equivalents is chosen. Supnormcontinuity cannot be defined in this general setting. The implications of supnorm-continuity, used in the analysis, must therefore be imposed explicitly. First, simple-continuity is re- 
quired, which is similarly defined as in the main text (endowing each finite product of outcome spaces with the product topology). Second, pointwise monotonicity is required. Third, the existence of a certainty equivalent is imposed for each act. Fourth and finally, a "simple-act denseness" condition is imposed to guarantee that the $V$-value of an act is indeed the infimum of the $V$-values of pointwise dominating simple acts, as well as the supremum of pointwise dominated simple acts: Simple-act denseness holds if, for all acts $f \succ h$, there exists a simple act $a$ such that $f \succ a \succ h$, where $a$ dominates $h$ pointwise, and a simple act $b$ such that $f \succ b \succ h$ where $b$ is pointwise dominated by $f$. The same condition is defined correspondingly for the representing functional $V$.

THEOREM 3.16 Let the Structural Assumption 3.1 hold, with the following modifications: The outcome set need not be $I R$ but can be any connected topological space. Assume that $S$ contains no atorns. Then the following two statements are equivalent:

(i) $\succcurlyeq$ is represented by a functional $V: \mathcal{F} \rightarrow \mathbb{R}$ that is additive, i.e., there exist functionals $V_{A}$ defined on the restrictions of acts to $A$ such that

$$
V(f)=\sum_{j=1}^{n} V_{A_{j}}\left(f_{A_{j}}\right)
$$

for each finite partition $\left\{A_{1}, \ldots, A_{n}\right\}$ of $S$. For each $A, V_{A}(x)$ (for outcome/constant act $x$ ) is continuous in $x$ and either constant or strictly increasing in $x$, and $V$ satisfies pointwise monotonicity and simple-act denseness.

(ii) $\succcurlyeq$ is a strictly monotonic weak order that satisfies pointurise monotonicity, simplecontinuity, the existence-of-certainty-equivalent condition, simple-act denseness, and the sure-thing principle.

The uniqueness for (i) is the same as in Theorem 3.11. The modifications of Observation 3.15 , except (b), also hold true for this theorem (in $(\mathrm{g})$, let any nonmaximal outcome $\beta$ play the role of zero).

The similar adaptation of Theorem 3.12 is more easily formulated because pointwise continuity can still be defined. The result refers to the order topology on the outcome set, and additionally needs topological separability.

THEOREM 3.17 Theorem 3.12 also holds true for general outcome sets with a connected separable order topology. 
Ordinal state independence is entailed by strict monotonicity and pointwise monotonicity. It is argued in the main text that in the case of monetary outcomes there is little interest in violations of ordinal state independence. This is different for general outcomes. If outcomes are commodity bundles, for instance, then ordinal state dependence may be economically meaningful (Karni 1993a, Section 4.2). The extension of the present representation to that case, in a way that avoids the generality of Example 3.4, is a topic for future research.

\subsection{Appendix A: Proofs of Results in Sections 3.1- 3.6}

Proof of Proposition 3.3. A partition of $S$ is essential if it contains at least three nonnull events. Note that any refinement of an essential partition is again essential because a partition of a nonnull event must itself contain at least one nonnull event. Obviously, an essential partition exists because of nonatomicity. To any essential partition $\pi=$ $\left\{A_{1}, \ldots, A_{n}\right\}$, we can apply Theorem 3.2 to obtain a continuous additive representation $\sum_{j=1}^{n} x_{j} 1_{A_{j}} \mapsto \sum_{j=1}^{n} V_{A_{j}}^{\pi}\left(x_{j}\right)$. For all essential partitions $\pi$, we can set $V_{A_{j}}^{\pi}(0)=0$ for all $A_{j}$ and $\sum_{j=1}^{n} V_{A_{j}}^{\pi}(1)=1$. (Dependence of $n$ on $\pi$ is not expressed in the notation.) By considering, for any two essential partitions, the common refinement, it now follows from the uniqueness result of Theorem 3.2 that the additive representations of different essential partitions coincide on common domain. This also implies that, for each event $A$, the function $V_{A}^{\pi}$ is the same for every essential partition $\pi: V_{A}^{\pi}(x)$ is the value of $x 1_{A}$ in any (other) essential partition that contains $A$ or a number of events $A_{j}$ whose union is $A$. Hence, we can drop the superscript $\pi$ in $V_{A}^{\pi}$ and write $V_{A} \cdot \sum_{j=1}^{n} x_{j} 1_{A_{j}} \mapsto \sum_{j=1}^{n} V_{A_{j}}\left(x_{j}\right)$ represents $\succcurlyeq$, first only for simple acts that are measurable with respect to a same essential partition of $S$, next for all simple acts because for each pair of simple acts there is an essential partition with respect to which both acts are measurable.

By Theorem 3.2, all functions $V_{A}$ are continuous. Obviously, if $A$ is mull, then $V_{A}$. is constant. If $A$ is nonnull, then strict monotonicity of $\succcurlyeq$ implies that $V_{A}$ is strictly increasing.

For the uniqueness result, it is obvious that any $W$ as described can be substituted for $V$. Next, it is assumed that $W$ is additively decomposable as desciibed and represents $\succcurlyeq$. By Wakker (1988), $W$ and each $W_{A}$ are continuous. We can replace each $V_{A}$ by $V_{A}-V_{A}(0)$ and each $W_{A}$ by $W_{A}-W_{A}(0)$, that is, it can be assumed that $V_{A}(0)=0=W_{A}(0)$ for each 
event $A$. We can divide each $V_{A}$ by $V(1)$ and each $W_{A}$ by $W(1)$, i.e., it can be assumed that $V(1)=1=W(1)$. It can be inferred from the proof that $W_{A}=V_{A}$ for all events $A$. In particular, $W=V$. For completeness, explicit expressions are given for the parameters $\sigma$ and $\tau$ that result from the preceding operations and that imply additivity of $\tau$ :

$$
\sigma=\frac{W(1)-W(0)}{V(1)-V(0)}: \quad \tau_{A}=W_{A}(0)-\sigma V_{A}(0)
$$

Proof of Lemma 3.5. Additivity of $V$ immediately implies the form described in the lemma. We now assume the form in the lemma and derive additivity. In order to do so, the definition of $V_{A}^{*}$ is extended from the acts that are constant on $A$ to arbitrary, nonconstanit, restrictions of simple acts to $A$. For any simple act $f$ and event $A$, there exist a partition $\left\{B_{1}, \ldots, B_{m}\right\}$ of $A$ such that $f$ is constant and is equal to, say, $f_{j}$, on each $B_{j}$. Define $V_{A}\left(f_{A}\right)=\sum_{j=1}^{m} V_{B_{j}}^{*}\left(f_{j}\right), V_{A}$ extends $V_{A}^{*}$ to nonconstant simple acts on $A$.

Proof of Lemma 3.8. First an immediate corollary of the representation in Proposition 3.3 is noted.

Corollary 3.18 On $\mathcal{F}^{s}, V$ and $\succcurlyeq$ satisfy pointwise monotonicity.

Take any $f \in \mathcal{F}$. Because all acts are bounded in this analysis, there exist outcomes $x, y$ such that $x \geqslant f(s) \geqslant y$ for all states $s$. Note that there is no monotonicity assumption to guarantee that $x \succcurlyeq f \succcurlyeq y$. Instead, we derive these preferences from supnorm-continuity in conjunction with strict monotonicity. It is well-known that $\mathcal{F}^{s}$ is supnorm dense in $\mathcal{F}$, and that we can find sequences of simple acts $g^{j}$ and $h^{j}$ such that both sequences converge in supnorm to $f$ and $g^{j}(s) \geqslant g^{j+1}(s) \geqslant f(s) \geqslant h^{j+1}(s) \geqslant h^{j}(s)$ for all states s. We can "truncate" every $g^{j}$ at the outcomes above $x$ and every $h^{j}$ at the outcomes below $y$, i.e., it can be assumed that $x \geqslant g^{j}(s) \geqslant h^{j}(s) \geqslant y$ for all states $s$. According to Corollary $3.18, x \succcurlyeq g^{j} \succcurlyeq h^{j} \succcurlyeq y$ for all $j$. As a consequence of supnorm-continuity, $x \succcurlyeq f \succcurlyeq y$ follows. Consequently, the sets $\{z \in \mathbb{R}: z \succcurlyeq f\}$ and $\{z \in \mathbb{R}: z \preccurlyeq f\}$ are nonempty. They are closed by supnorm-continuity. Because of connectedness of $I R$, their intersection must contain at least one element. (Because of strict monotonicity, the intersection can contain at most one element.) The element in that intersection is the certainty equivalent of $f$.

LEMMA $3.19 \mathrm{~V}$, defined after Lemma 3.8, satisfies supnorm-continuity. 
Proof. Consider $\{f \in \mathcal{F}: V(f) \geqslant \alpha\}$ for some real $\alpha$. It will be shown that the set is closed. That is obvious if $\alpha$ is larger or smaller than any value in $V(\mathcal{F})$. In the other case, there exists a constant act $\mu$ such that $V(\mu)=\alpha$ because, by Lemma 3.8, $V(\mathcal{F})=V(\mathbb{R})$ and the latter is connected by continuity (Proposition 3.3). Now $\{f \in \mathcal{F}$ : $V(f) \geqslant \alpha\}=\{f \in \mathcal{F}: f \geqslant \mu\}$, which is closed because of continuity of $\succcurlyeq$. Similarly, $\{f \in \mathcal{F}: V(f) \leqslant \alpha\}$ is closed. Continuity of $V$ follows.

Proof of Lemma 3.9. Pointwise monotonicity on $\mathcal{F}^{s}$ follows from Proposition 3.3 . Assume $f(s) \geqslant g(s)$ for all $s$, for two general (possibly nonsimple) acts $f, g$. It is proved that $f \succcurlyeq g$. Take a sequence $a^{j}$ of simple acts converging pointwise from above to $f$ such that $f(s)+1 / j \geqslant a^{j}(s) \geqslant a^{j+1}(s) \geqslant f(s)$ for all $s$ and $j$. Similarly, take a sequence $b^{j}$ of simple acts converging pointwise from below to $g$, i.e., $g(s) \geqslant b^{j+1}(s) \geqslant b^{j}(s) \geqslant g(s)-1 / j$ for all $s$ and $j$. We have $a^{j}(s) \geqslant b^{j}(s)$ for all states $s$, therefore, because of pointwise monotonicity on $\mathcal{F}^{s}$ as previously established, $V\left(a^{j}\right) \geqslant V\left(b^{j}\right)$ for all $j$. Because of supnormcontinuity, $V(f)=\lim V\left(a^{j}\right)$ and $V(g)=\lim V\left(b^{j}\right)$, therefore $V(f) \geqslant V(g)$.

Proof of Lemma 3.10. Assume that $V_{A}$ is defined on the restrictions of all simple acts to $A$ (as $V_{A^{*}}$ in Lemma 3.5). Thus, $V$ is additive on $\mathcal{F}^{s}$. Note that $V(f)-V(0)=$ $\sum_{j=1}^{n}\left(V_{A_{j}}\left(f_{A_{j}}\right)-V_{A_{j}}\left(0_{A_{j}}\right)\right)$ for all simple acts $f$ and partitions $\left\{A_{1}, \ldots, A_{n}\right\}$. Therefore, we can define $W_{A}=V_{A}-V_{A}\left(0_{A}\right)$ for all events $A$ and restrictions of simple acts to $A$, and $W=V-V(0)$ for all acts including the nonsimple ones. Then $W$ inherits all the relevant properties of $V$, in particular additivity on $\mathcal{F}^{s}$, and in addition $W$ and all $W_{A}$ s assign 0 to the act that is constant 0 . The definition of $W_{A}$ is extended to restrictions of nonsimple acts by defining $W_{A}\left(f_{A}\right)=W\left(f_{A} 0\right)$ for all acts $f$. Note, that this definition agrees with the original one on $\mathcal{F}^{s}$. It is shown that with these definitions, $W$ also satisfies additivity for the nonsimple acts. Let $f$ be an arbitrary nonsimple act, $\left\{A_{1}, \ldots, A_{n}\right\}$ an arbitrary finite partition of $S$, and $f^{j}$ a sequence of simple acts converging to $f$ in supremum norm. Taking limits for $j \rightarrow \infty$, and explaining the equalities later,

$$
\begin{aligned}
W(f)= & \lim W\left(f^{j}\right)=\lim \sum_{i=1}^{n} W_{A_{i}}\left(f_{A_{i}}^{j}\right)=\sum_{i=1}^{n} \lim W_{A_{i}}\left(f_{A_{i}}^{j}\right)=\sum_{i=1}^{n} \lim W\left(f_{A_{i}}^{j} 0\right)= \\
& \sum_{i=1}^{n} W\left(f_{A_{i}} 0\right)=\sum_{i=1}^{n} W_{A_{i}}\left(f_{A_{i}}\right) \text { results. }
\end{aligned}
$$

Here the first equality follows from supnorm-continuity of $W$, the second from additivity of $W$ on $\mathcal{F}^{s}$, the third mainly from boundedness of acts, the fourth from the definition of 
$W_{A_{i}}$, the fifth because $f^{j}{ }_{A_{i}} 0$ converges to $f_{A_{2}} 0$ in supnorm (implied by convergence of $f^{j}$ to $f$ ) and $W$ is supnorm-continuous, and the final equality by definition of $W_{A_{1}}$. Thus, additivity of $W$ has been established. Finally, additivity of $V$ is established. We already have $V=W+V(0)$ for all acts and $V_{A}=W_{A}+V_{A}\left(0_{A}\right)$ for all simple acts. Define $V_{A}$ similarly for all nonsimple acts. Additivity of $V$ now follows from additivity of $W$.

Proof OF THEORFM 3.11. The implication (i) $\Rightarrow$ (ii) is elementary (the sure-thing principle follows from Observation 3.6). Next suppose (ii) holds; (i) is derived. Supnormcontinuity of $\succcurlyeq$ implies simple-continuity because for each $n$ the supnorm topology coincides with the Euclidean topology on $\mathbb{R}^{n}$. Therefore, the representation in Proposition 3.3 is cbtained, with each $V_{A}(x)$ continuous in $x$. By Lemma 3.5, $V$ is additive on $\mathcal{F}^{s}$. $V$ is extended to all of $\mathcal{F}$ as described after Lemma 3.8. It represents the preference relation, is supnorm-continuous by Lemma 3.19, and is additive by Lemma 3.10. $V_{A}$ is constant if $A$ is null and, because of strict monotonicity, $V_{A}(x)$ is strictly increasing in outcomes/constant acts $x$ if $A$ is nomull. This proves the implication (ii) $\Rightarrow$ (i). By Lemma 3.9, $V$ in (i) satisfies pointwise monotonicity. If (ii) holds, then (i) holds with $V$ pointwise monotonic, which implies that $\succcurlyeq$ is pointwise monotonic. Continuity of $V_{A}(x)$ is ensured by Proposition 3.3 and its uniquiness result.

Finally, the uniqueness result is discussed, which is similar to the result of Proposition 3.3 , the main exception being that the functions $V_{A}$ and $W_{A}$ apply not only to outcomes/constant acts on $A$ but also to nonconstant restrictions of acts to $A$. The proof is similar to the proof of the uniqueness result in Proposition 3.3. Note that, once the normalized functionals $V$ and $W$ agree on all simple acts, they also agree on the nonsimple acts through their certainty equivalents.

\subsection{Appendix B: Proofs of Results in Section 3.7}

Proof of Theorem 3.12. For a countably additive measure, the integral of a positive function over a nonnull set is positive. Further, if the integral of one function over every nonnull set dominates the integral of a second function, then the first function dominates the second almost everywhere. These two facts are applied on a number of occasions without further mention.

We first assurne Statement (i) and prove Statement (ii). Weak ordering is immediate and the sure-thing principle follows from additivity of the representation, as in Observation 3.6. For strict monotonicity, assume that $x>y$ and that $A$ is not null. The latter implies, 
by the integral representation, that $\mu(A)>0$ must hold. For each $s \in A, U_{s}(x)>U_{s}(y)$. Because of countable additivity, $\int_{A} U_{s}(x) d \mu-\int_{A} U_{s}(y) d \mu>0$. This implies that $x_{A} f \succ y_{A} f$ for all acts $f$, in particular those which are simple. Strict monotonicity holds. Finally, it is well-known that continuity of a representing function implies the same continuity of $\succcurlyeq$. Statement (ii) has been proved.

In the rest of this proof, Statement (ii) is assumed and (i) and the uniqueness results are derived.

LEMMA $3.20 \succcurlyeq$ satisfies supnorm continuity.

Proor. If a sequence of acts converges to a limiting act in supnorm, then it also converges pointwise. The boundedness of all acts in this analysis and therefore of the limiting act in particular, as well as the supnorm convergence of the sequence, imply that the sequence is uniformly bounded. By pointwise continuity, the sequence of acts converges preferencewise, which is what supnorm continuity requires.

By the lemma, all conditions of Statement (ii) of Theorem 3.11 are satisfied. Thus Statement (i) of that theorem is also satisfied, yielding the additive representation $V$.

LEMMA $3.21 V$ satisfies pointwise continuity.

Proof. Let $f^{j}$ be uniformly bounded and converge to $f$ pointwise. First assume, for contradiction, that for a subsequence of the $f^{j}$ s, the $V$ value would always exceed $V(f)+\epsilon$ for a fixed positive $\epsilon$. Because of continuity, we can find a constant act $x$ with $V(f)<$ $V(x)<V(f)+\epsilon$. Pointwise continuity of $\succcurlyeq$, and $f^{j} \succcurlyeq x$ for all $f^{j}$ from the subsequence, imply that $f \succcurlyeq x$, contradicting $V(f)<V(x)$. Hence, no such subsequence can exist. Similarly, no subsequence can exist for which the $V$ value would always be below $V(f)-\epsilon$ for a fixed positive $\epsilon$. Hence, $V\left(f^{j}\right)$ must converge to $V(f)$ and $V$ is pointwise continuous. $\nabla$

$V$ is normalized by subtracting $V_{A}(0)$ from all functions $V_{A}$, then dividing all those functions by the positive scale factor $V(1)$. In other words, it is assumed henceforth that $V_{A}(0)=0$ for all events $A$ and $V(1)=1$. Define $\mu(A)=V\left(1_{A}\right)$ for all $A$. It was also possible to take any other outcomes $\alpha>\beta$, and define an alternative $\mu^{\prime}$ by $\mu^{\prime}=\frac{V\left(\alpha 1_{\Lambda}-\beta 1_{\Lambda c}\right)}{V(\alpha)-V(\beta)}$. The choice of $\alpha=1$ and $\beta=0$ was arbitrary.

LEMMA 3.22 For any act $f, \mu_{f}(A)=V\left(f 1_{A}\right)$ (with $\left.f 1_{A}=f_{A} 0\right)$ is a countably additive signed measure. In particular, $\mu(A)=V\left(1_{A}\right)$ is a countably additive measure. 
Proof. $\mu_{f}$ is finitely additive because $V$ is additive (hence, $\mu_{f}(\theta)=0$ ). For countable additivity, let $\left(A_{j}\right)_{j=1}^{\infty}$ be ar sequence of disjoint events and let $A$ be their union. Then $f 1_{\cup_{j=1}^{n} A}$ converges to $f 1_{A}$ pointwise. The sequence is also uniformly bounded because $f$ is bounded. The $V$ values of the sequence must converge, because of pointwise continuity (Lemma 3.21). This means that $\sum_{j=1}^{n} \mu_{f}\left(A_{j}\right)$ converges to $\mu_{f}(A)$, and countable additivity holds. $\mu$ is a measure because pointwise monotonicity implies that it is nonnegative. (Note that also $\mu(S)=1$.)

The following lemma immediately follows from strict monotonicity.

LEMMA $3.23 \mu(A)=0$ if and only if $A$ is null.

Because of this lemma, "null" event can equivalently refer to the preference definition as well as to the measure $\mu$. In the remainder of this proof, a.e. (almost everywhere) means that a condition holds up to a null event.

We next turn to the most complicated part of the proof, the derivation of the statedependent utility $U_{s}$. On the basis of Lemma 3.23 and Observation 3.15(d), each $\mu_{f}$ is absolutely continuous with respect to $\mu$. According to the theorem of Radon-Nikodym, we can define a density $\delta_{f}$, unique up to a null event, such that $V_{A}(f)=\int_{A} \delta_{f}(s) d \mu$ for all $A$.

First a brief preview of the proof. We will relate the definition of state-dependent utility $U_{s}(\alpha)$ to the constant act $\alpha$ by defining $U_{s}(\alpha)=\delta_{\alpha}(s)$, for all $s, \alpha$. It then remains to be verified that also for the nonconstant acts $f, \delta_{f}(s)=U_{s}(f(s))$. That is, $\delta_{f}$ should satisfy a kind of "separability" ( $\simeq$ sure-thing principle) in the sense that $\delta_{f}(s)$ depends on $f$ only through $f(s)$, and is independent of $f$ on $\{s\}^{c}$. That "state-wise" separability is ensured for simple acts by the "event-wise" separability induced by the additive representation, as will be demontrated in Lemma 3.25. An additional complication can arise for nonsimple acts. This complication is illustrated in Example 3.24. Following the example, state-dependent utilities are defined such that the complication is avoided. Only after that will the integral representation be established, first for simple acts, then for general.

At this point an explanation of the previously mentioned complication is in order. Each $\delta_{f}$ is only defined a.e. Therefore, $\delta_{f}$ can be considered an equivalence class of functions differing only on a null event. An appropriate representative from each such equivalence class will have to be chosen with some care when nonsimple acts are involved. 


\section{EXAMPLE 3.24}

Assume that $S=[0,1]$ and that $\geqslant$ maximizes expected value. For each real $\alpha$ a representative $\delta_{\alpha}(s)=\alpha$ has been chosen, with the only exception being that for each $0 \leqslant \alpha \leqslant 1$ we have $\delta_{\alpha}(s)=0$ for $s$ in the null event $\{\alpha\}$. Then $U_{s}(\alpha)=0$ for $\alpha=s$ and the act $f(s)=s$ would be assigned the state-dependent expected utility value 0 , even though $f$ is strictly preferred to the constant 0 act. Thus, state-dependent expected utility would not represent preference. In addition, each $U_{s}$ would not be strictly increasing.

For each real $\alpha \geqslant \beta$ and each event $A, \int_{A} \delta_{\alpha}(s) d \mu=V_{A}(\alpha) \geqslant V_{A}(\beta)=\int_{A} \delta_{\beta}(s) d \mu$, hence

$$
\alpha \geqslant \beta \Rightarrow \delta_{\alpha}(s) \geqslant \delta_{\beta}(s) \text { a.e. }
$$

We first ensure that the implication in (3.7) holds everywhere. There are countably many pairs of rational numbers, hence there is one null event (the union of countably many) such that outside that event, (3.7) holds everywhere for all pairs of rational numbers. We can therefore choose the $\delta_{\rho}$ s for rational numbers $\rho$ such that (3.7) holds everywhere for the rational numbers. (E.g., we can redefine $\delta_{\rho}(s)=\rho$ on the previously mentioned null event.)

For each real number $\alpha$, there is one null event (the union of countably many) such that (3.7) holds outside the null event for all rational $\beta$. Hence, we can choose each $\delta_{\alpha}$ such that (3.7) holds for all real $\alpha$ and rational $\beta$. Similarly, we can let (3.7) hold for all rational $\alpha$ and real $\beta$ as well. Given that for each real $\alpha>\beta$ we can find a rational number between them, we conclude that (3.7) now holds everywhere for all real numbers $\alpha, \beta$.

For each $s$ and outcome $\alpha$, we define $U_{s}(\alpha)=\delta_{\alpha}(s)$. By (3.7) as just extended, this function is nondecreasing in $\alpha$ for each $s$. We prove that the function is strictly increasing a.e. by showing that a violation would translate into a violation of the same condition for $V_{A}$ for a nonnull event $A . V$ being nondecreasing is often used in the following reasonings.

To prove that $U_{s}$ is strictly increasing a.e., consider, for any rational $\rho>\rho^{\prime}, A=$ $\left\{s \in S: U_{s}(\rho)=U_{s}\left(\rho^{\prime}\right)\right\}$. Then $V_{A}(\rho)=V_{A}\left(\rho^{\prime}\right)$, which implies that $A$ must be null, $V_{A}$ being strictly increasing for each nonnull $A$. As there are only countably many pairs of rational numbers $\rho>\rho^{\prime}, U_{s}$ is strictly increasing in the rational numbers, hence in the real numbers, a.e. We may assume that $U_{s}$ is strictly increasing for all $s$.

LEMMA 3.25 If $g$ is simple, then for each event $A, V_{A}(g)=\int_{A} U_{s}(g) d \mu$. 
Proof. For $g, A$, there is a partition $\left\{A_{1}, \ldots, A_{n}\right\}$ of $A$ such that $g 1_{A}=\sum_{j=1}^{n} g_{j} 1_{A_{j}}$ for outcomes $g_{j}$. Now $V_{A}(g)=\sum_{j=1}^{n} V_{A_{j}}\left(g_{j}\right)=\sum_{j=1}^{n} \int_{A_{j}} \delta_{g_{j}}(s) d \mu=\sum_{j=1}^{n} \int_{A_{j}} U_{s}\left(g_{j}\right) d \mu=$ $\int_{A} U_{s}(g) d \mu$, which is what should be proved.

Finally, consider a general act $f$. For each event $A$ and simple act $g$ that is pointwise dominated by $f$,

$$
\int_{A} \delta_{f}(s) d \mu=V_{A}(f) \geqslant V_{A}(g)=\int_{A} \delta_{g}(s) d \mu=\int_{A} U_{s}(g(s)) d \mu .
$$

Because each $U_{s}$ is strictly increasing and because of pointwise dominance, also $\int_{A} U_{s}(f(s)) d \mu \geqslant \int_{A} U_{s}(g(s)) d \mu$. Reversed inequalities hold both for $\int_{A} \delta_{f}(s) d \mu$ and for $\int_{A} U_{s}(f(s)) d \mu$ if $g$ pointwise dominates $f$. Because of supnorm continuity of $V_{A}$, the upper and lower bounds derived from pointwise dominating/dominated simple acts $g$ are tight and hence, for each event $A, \int_{A} U_{s}(f(s)) d \mu$ and $\int_{A} \delta_{f}(s) d \mu$ are bounded by the same upper and lower bounds, i.e., they are identical. This implies that $\delta_{f}(s)=U_{s}(f(s))$ a.e., that is, $\delta_{f}(s)=U_{s}(f(s))$ can be chosen for all $s$, and $V(f)=\int_{S} U_{s}(f(s)) d \mu$ holds for all acts $f$. Statement (i) has been proven.

Finally, the uniqueness results of the theorem are established. It is obvious that $\left(U_{s}\right)_{s \in S}, \mu$ can be replaced by $\left(U_{s}^{*}\right)_{s \in S}, \mu^{*}$ as described in the theorem. Let us now explain that no other substitutions are possible. Assume that $\left(U_{\mathrm{s}}\right)_{s \in S}$ and $\mu$ can be replaced by $\left(U_{s}^{*}\right)_{s \in S}$ and $\mu^{*}$. Absolute continuity follows from the following lemma, which extends Lemma 3.23 to general $\mu^{*}$, not just the special $\mu$ as constructed in this proof.

LEMMA $3.26 \mu^{*}(A)=0$ if and only if $A$ is null.

Proof. If $\mu^{*}(A)=0$ then $A$ is null. Assume therefore that $\mu^{*}(A)>0$. It is shown that $A$ is nonnull. Take outcomes $x>y$. Because $U_{s}^{*}$ must be strictly increasing for each $s$ and because of countable additivity, $\int_{A} U_{s}^{*}(x) d \mu^{*}>\int_{A} U_{s}^{*}(y) d \mu^{*}$ and $A$ is nonnull.

Lemmas 3.23 and 3.26 imply that $\mu$ and $\mu^{*}$ have the same null events and are, therefore, absolutely continuous with respect to each other. Next we turn to the uniqueness result regarding $U$. Let $\delta$ be the Radon-Nikodym density function of $\mu$ with respect to $\mu^{*}$. We can replace $\mu^{*}$ by $\mu$ and divide $U_{s}^{*}$ by $\delta(s)$, that is, it can be assumed that $\mu^{*}=\mu$; note that $\delta$ is zero only on a null set which can be ignored. The constant $U_{s}(0)$ can be subtracted from $U_{s}(\cdot)$ and the constant $U_{s}^{*}(0)$ from $U_{s}^{*}(\cdot)$ for each $s$, i.e., it can be assumed that $U_{s}(0)=0=U_{s}^{*}(0)$ for all $s$. We can divide each $U_{t}$ by the positive scale factor $\int_{S} U_{s}(1) d \mu$ 
and each $U_{t}^{*}$ by the positive scale factor $\int_{S} U_{s}^{*}(1) d \mu$, i.e., it can be assumed that these positive scale factors are 1.

It suffices to show that $U_{s}$ and $U_{s}^{*}$ agree a.e. after the preceding modifications. Consider, for any $A$ such that both $A$ and $A^{c}$ are nonnull, the two-dimensional set of acts of the form $x 1_{A}+y 1_{A^{c}}$. Here, $\int_{A} U_{s}(x) d \mu+\int_{A^{c}} U_{s}(y) d \mu=V_{A}(x)+V_{A}(y)$ provides an additive and continuous representation of preference. Such a representation also results with $U^{*}$ instead of $U$. Because the two representations have been renormalized to agree at the constant 0 and 1 acts, they must agree by the common uniqueness results of additive conjoint measurement (Karni \& Safra, 1998). Hence, the integrals of $U_{s}$ and $U_{s}^{*}$ over all such events $A$ agree. A similar conclusion holds for nonnull events $A$ for which $A^{e}$ is null (e.g., partition $A$ into two nonnull events, etc.). It follows that $U_{s}$ and $U_{s}^{*}$ must coincide a.e., which completes the proof of the theorem.

The following lemma demonstrates that pointwise continuity is not overly restrictive because continuity of each state-dependent utility implies pointwise continuity. Whether the reversed implication holds, i.e., whether continuity of the functions $U_{s}$ in Statement (i) of Theorem 3.12 holds (after appropriate modification on null events), is an open question.

LEMMA 3.27 Replacing in (ii) the pointwise continutity of the integral by continuity of each $U_{s}$ implies pointwise continuity of preference (which is equivalent to that condition for the integral) and, thus, all of (i) and (ii).

Proof. Assume that $x \succcurlyeq f_{j}(s) \succcurlyeq y$ for all $j$ and $s$ and that $f_{j}(s)$ converges to $f(s)$ for all $s$. Then also $U_{s}\left(f_{j}(s)\right)$ converges to $U_{s}(f(s))$ for all $s . U_{s}(x)$ and $U_{s}(y)$ are integrable upper and lower bounds, hence $\int_{S} U_{s}\left(f_{j}(s)\right) d \mu$ converges to $\int_{S} U_{s}(f(s)) d \mu$ by the dominated convergence theorem of Lebesgue (Dunford \& Schwartz, 1958, Corollary I.III.6.16). That is, the representing integral satisfies a pointwise continuity condition that implies pointwise continuity of $\succcurlyeq$.

\subsection{Appendix C: Proof of Observation 3.15}

(a) The proof of (ii) $\Rightarrow$ (i) in Theorem 3.11 never used more of the sure-thing principle.

(b) This follows from the equality $V_{A}\left(f_{A}\right)=V\left(f_{A} 0\right)-k$ for the constant $k=V_{A^{c}}\left(0_{A^{c}}\right)$, and from supnorm continuity of $V$. 
(c) With additivity of $V$ only on $\mathcal{F}^{s}$, Statement (i) still implies Statement (ii), in particular because Statement (ii) requires the sure-thing principle only on $\mathcal{F}^{s}$ (see (a)). Statement (ii), in turn, implies all of Statement (i), in particular additivity of $V$ (mainly established in Lemma 3.10).

(d) Because of the sure-thing principle, the preference between $g_{A} f$ and $h_{A} f$ is the same as the preference between $g_{A} 0$ and $h_{A} 0$. Because of boundedness of acts, we can take outcomes $x, y$ such that $x \geqslant g(s) \geqslant y$ and $x \geqslant h(s) \geqslant y$ for all $s \in A$. Then $x_{A} 0 \succcurlyeq g_{A} 0 \succcurlyeq y_{A} 0$ and $x_{A} 0 \succcurlyeq h_{A} 0 \succcurlyeq y_{A} 0$, because of pointwise monotonicity. But also $x_{A} 0 \sim y_{A} 0$, because both acts are simple and therefore this indifference follows from event $A$ being null. It also follows that $g_{A} 0$ and $h_{A} 0$ must be indifferent to these two simple acts and hence to each other. This is likewise true of $g_{A} f$ and $h_{A} f$, because of the sure-thing principle.

(e) This follows from $x_{A} 0 \succ y_{A} 0$ and the sure-thing principle.

(f) The proofs require no modifications for this result. (In particular, the proof of Proposition 3.3 has been written so that it also applies to this case.)

(g) When the domain under consideration is a subset of $\mathcal{F}^{s}$, then the definition of the various conditions such as the sure-thing principle are obviously restricted to the domain under consideration. Note that, by (a) of Observation 3.15, the sure-thing principle is only needed on $\mathcal{F}^{s}$. The proofs require no other modifications for this case.

\subsection{Appendix D: Proof of Theorems 3.16 and 3.17}

In this appendix, the proofs of the preceding results for connected topological outcome spaces are presented.

Proof of Theorem 3.16. The implication (i) $\Rightarrow$ (ii) is straightforward, the existence of certainty equivalents following from continuity and pointwise monotonicity of $V$, and connectedness of the outcome set, as in the proof of Lemma $3.8(x \geqslant f \succcurlyeq y$ there follows immediately from pointwise monotonicity of $V$ ). Next (ii) is assumed and (i) is derived. This is demonstrated in a similar manner as the implication (ii) $\Rightarrow$ (i) in Theorem 3.11 and goes through a number of steps. 
(STEP 1) Theorem 3.2 needs no modification. Note, however, that Debreu (1960) also assumed topological separability of the outcome space. It was pointed out by Krantz et al. (1971) that this assumption can be dropped (their proof was supplemented by Wakker, 1988) and by Wakker (1989b, Theorem III.6.6 and Remark A3.1)).

(Step 2) In the case of Propusition 3.3 and the subsequent results, one difference is that no natural $U$ and 1 outcomes are given. The remedy is easy: take any outcomes $\alpha \succ \beta$, then "normalize" $V$ by setting $V_{A}(\beta)=0$ for all $A$ and $V(\alpha)=1$. Subsequently, $\beta$ plays the role of the 0 -outcome, $\alpha$ the role of the 1-outcome. Other than that, the results in Section 3.5 need no modification.

(STEP 3) The result of Lemma 3.8 is now assumed explicitly, and Lemma 3.9 immediately follows from the similar assumption for preference.

(STEP 4) The proof of Lemma 3.10 requires a more elaborate revision, concerning additivity of the functional $W$ constructed there. We cannot invoke supnorm continuity at this point, and instead must invoke simple-act denseness and the other assumptions. Let $f$ be a nonsimple act and $\left\{A_{1}, \ldots, A_{n}\right\}$ a partition of $S$. It is shown that $W(f)=$ $\sum_{i=1}^{n} W_{A_{i}}\left(f_{A_{x}}\right)$. Let $\gamma$ be a certainty equivalent of $f$. First, sequences $a^{j}, b^{j}$ of simple acts are constructed such that (1) with $W$ the normalized version of $V$ that satisfies $W_{A}(\beta)=0$ for all $A$ and $W(\alpha)=1$, the result is

$$
W(f)-1 / j \leqslant W\left(b^{j}\right) \leqslant W(f)=W(\gamma) \leqslant W\left(a^{j}\right) \leqslant W(f)+1 / j
$$

for all $j,(2) f$ dominates each $b^{j}$ pointwise, and (3) each $a^{j}$ dominates $f$ pointwise. The construction of the $a^{j} \mathrm{~S}$ is described in detail. If $f$ is maximal, i.e., no act $g$ is strictly preferred to $f$ (this case can occur for general connected topological outcome spaces), then the certainty equivalent $\gamma$ of $f$ must be a maximal outcome and we simply take each $a^{j}$ equal to $\gamma$. Note that this dominates $f$ pointwise. If $f$ is not maximal, then there exists a strictly preferred act and the certainty equivalent thereof is also strictly preferred to $f$ and $\gamma$. Because of simple-continuity on $\mathcal{F}^{s}$ and connectedness, the $W$ image of outcomes is an interval. Hence, there must exist, for each $j$, a constant act $c^{j}$ which is between the certainty equivalent strictly preferred to $f$, and $\gamma$, such that $W(\gamma)=W(f)<W\left(c^{j}\right) \leqslant$ $W(f)+1 / j$. Now simple-act denseness is invoked, which implies the existence of a simple act $a^{j}$ that dominates $f$ pointwise and satisfies $W(f) \leqslant W\left(a^{j}\right) \leqslant W\left(c^{j}\right) \leqslant W(f)+1 / j$. The acts $b^{j}$ are constructed similarly. We have now achieved the same kind of enclosure 
of $f$ between simple acts, from above and below, as under supnorm continuity for real outcomes.

Taking limits for $j \rightarrow \infty$, we get (explaining the equalities later)

$$
\begin{aligned}
W(f)= & \lim W\left(a^{j}\right)=\lim \sum_{i=1}^{n} W_{A_{i}}\left(a_{A_{i}}^{j}\right)=\sum_{i=1}^{n} \lim W_{A_{i}}\left(a_{A_{i}}^{j}\right)=\sum_{i=1}^{n} \lim W\left(a_{A_{i}}^{j} \beta\right)= \\
& \sum_{i=1}^{n} W\left(f_{A_{i}} \beta\right)=\sum_{i=1}^{n} W_{A_{i}}\left(f_{A_{i}}\right),
\end{aligned}
$$

establishing additivity of $W$.

The first equality follows from the definition of the acts $a^{j}$, the second follows from additivity of $W$ on $\mathcal{F}^{s}$ (Lemma 3.5), the third is elementary because of boundedness, the fourth follows from the definition of $W_{A_{i}}$, the fifth is explained later, and the sixth follows from the definition of $W_{A_{1}}$. For the fifth equality it is shown that $\lim _{j \rightarrow \infty} W\left(a^{j} A_{i} \beta\right)=$ $W\left(f_{A_{i}} \beta\right)$ for each $i$. This follows from the inequalities $\frac{2}{j} \geqslant W\left(a_{A_{i}}^{j} \beta\right)-W\left(\hat{f}_{A_{i}} \beta\right) \geqslant 0$ for all $j$, which is derived from the following inequalities, explained thereafter:

$$
\frac{2}{j} \geqslant W\left(a^{j}\right)-W\left(b^{j}\right) \geqslant W\left(a^{j}{ }_{i} \beta\right)-W\left(b^{j}{ }_{A_{t}} \beta\right) \geqslant W\left(a_{A_{t}}^{j} \beta\right)-W\left(f_{A_{i}} \beta\right) \geqslant 0
$$

for all $j$. First, note that all differences in the inequality are nonnegative because the left act always dominates the right act pointwise. The first inequality follows from the construction of the simple acts $a^{j}$ and $b^{j}$, which both differ by less than $\frac{1}{j}$ from $f$ in $W$ units. For the second inequality, we invoke the additive representation on $\mathcal{F}^{s}$ with respect to the partition $\left\{A_{1}, \ldots, A_{n}\right\}$. The term corresponding to event $A_{i}$ is the same for the leftand right-hand side, for all other events the terms for the left-hand side are nonnegative, for the right-hand side they are. 0 . The third inequality follows because $f_{A_{i}} \beta$ dominates $b_{A_{1}} \beta$ pointwise. The fourth inequality follows because $\left(a^{j} A_{i} \beta\right)$ dominates $f_{A_{i}} \beta$ pointwise.

Thus, additivity of the functional $W$ has been proven. Other than that, the proof needs no adaptation.

(STEF 5) Next. we turn to the completion of the proof of the implication (ii) $\Rightarrow$ (i) in Theorem 3.16, by adapting the related proof in Theorem 3.11. Pointwise monotonicity and simple-act denseness are equivalent for preferences and for $V$. Supnorm continuity is not even defined now. 'This establishes the implication.

(STEP 6) The uniqueness results in Theorem 3.16 and the extensions provided in 
Observation 3.15 are proved in the same manner as in Theorem 3.11 (where again any $\alpha \succ \beta$ play the role of the outcomes 1 and 0 ).

Proof of Theorem 3.17. The implication (i) $\Rightarrow$ (ii) is again straightforward, hence we assume (ii) and derive (i). Pointwise continuity implies simple-continuity, hence we can invoke Theorem 3.2 and Proposition 3.3. For the proof of the following lemma, it is crurial that the outcome sct is endowed with the order topology.

\section{LEMMA 3.28 Simple-act denseness holds.}

Proof. Let $Y$ denote a countable dense subset of the outcome space; such a subset exists because of topological separability. We may assume that it contains a maximal and minimal outcome if such exist. For each act $f$ we can construct dominating simple acts $a^{j}$ and dominated simple acts $b^{j}$, respectively, that converge to $f$ pointwise, as follows. Write $Y=\left\{y_{1}, y_{2}, \ldots\right\}$. Let $z_{0} \in Y$ satisfy $z_{0} \succcurlyeq f(s)$ for all $s$. Such a $z_{0}$ exists because $f$ is bounded and $Y$ contains a maximal outcome if such exists. Let $\left\{z_{1}, \ldots, z_{j}\right\}$ be a reordering of $\left\{y_{1}, \ldots, y_{j}\right\}$ such that $z_{1} \succcurlyeq \cdots \succcurlyeq z_{j}$. Then $a^{j}=\sum_{i=0}^{j} z_{i} 1_{A_{1}}$ where $A_{i}=\{s \in$ $\left.S: z_{i} \succcurlyeq f(s) \succ z_{i+1}\right\}$ for all $i \leqslant j-1, A_{j}=\left\{s \in S: z_{j} \succcurlyeq f(s)\right\}$. Each $a^{j}$ dominates $a^{j+1}$ pointwise and they all dominate $f$ pointwise. They converge to $f$ pointwise because for each $s$ and $x \succ f(s)$, some $y_{k}$ will come between $x$ and $f(s)$ implying $x \succ y_{k} \geqslant a^{j}(s) \succcurlyeq f(s)$ for all $j \geqslant k$. The $b^{j}$ s are defined similarly. Both sequences of simple acts are uniformly bounded (the $a^{j} s$ are bounded by $\approx n$ and the lower bound of $f$ ), hence pointwise convergence implies preference-wise convergence due to pointwise continuity. That implies simple-act denseness.

The proof of Lemma 3.8 can now be adapted in a straightforward manner, using pointwise continuity instead of supnorm continuity, and using Lemma 3.28. Thus, for every act a certainty equivalent exists (possibly more). By pointwise continuity, the simple acts that converge to an act pointwise also converge in preference. Hence, $V$ (defined through certainty equivalents as in the main text) inherits pointwise monotonicity for all acts from pointwise monotonicity on the simple acts, i.e., Lemma 3.9 also holds. Now all conditions in Statement (ii) of Theorem 3.16 hold, therefore Statement (i) there also holds. From this point on, the proof of Theorem 3.12 from Lemma 3.21 onwards can be followed by fixing any $\alpha \succ \beta$ instead of $1>0$, taking a countable dense subset $Y$ instead of the rational numbers, and applying pointwise continuity instead of supnorm continuity. 


\subsection{Appendix E: Proof of Results in Section 3.8}

Proof of Theorem 3.13. If (i) holds, then for all $\lambda_{j}$ equal to $0, A_{j}$ is posterior-null. For all positive $\lambda_{j}$, the posterior preference relation conditional on $A_{j}$ is represented by $V_{A_{j}}$, i.e., it is identical to the prior preference relation conditional on $A_{j}$. Hence (i) implies (ii). Next assume (ii). Assume that the posterior preference relation, satisfying all requirements of Theorem 3.11 , is represented by $f \mapsto \sum_{j=1}^{n} W_{A_{j}}\left(f_{A_{j}}\right)$. We may assume that, for each event $A_{i} V_{A}(0)=0$ and $W_{A}(0)=0$. If $A_{j}$ is posterior-null then $W_{A_{j}}=0$ and we set $\lambda_{j}=0$. Next assume $A_{j}$ is not posterior-null. In this case, the prior and posterior preference relation, conditional on $A_{j}$, are the same. The crucial point in this proof is based on consideration of this preference relation on its own, and applying Theorem 3.11 to it. Thus $S^{\prime}=A_{j}$ is considered to be a state space. Note that it contains no atoms. The preference relation satisfies all conditions in Theorem 3.11 , and $V_{A_{j}}$ and $W_{A_{j}}$ are additive representations of it. From the uniqueness result in Theorem 3.11 and the scaling $V_{A}(0)=0$ and $W_{A}(0)=0$ for all events $A \subset A_{j}$, it follows that there exists a positive factor $\lambda_{j}$ such that $W_{A_{j}}=\lambda_{j} V_{A_{j}}$.

Proof of Theorem 3.14. Take any finite partition $\left\{A_{1}, \ldots, A_{n}\right\}$ containing at least two nonmull events. Then the clairn of the theorem, including expected utility representation, holds for acts that are, constant on each element of the partition, by Miyamoto \& Wakker (1996, Example 1.c). If expected utility holds on every finite partition, then in the presence of the other assumptions, it nust hold for all acts (Wakker, 1993a, Corollary 2.14; Wakker's assumption of truncation-continuity is vacuously satisfied in the present context where all acts are bounded). 


\section{Chapter 4}

\section{A Simple Axiomatization of}

\section{Rank-Dependent Utility and}

\section{Cumulative Prospect Theory with}

\section{Constant Proportional Risk Aversion}

\subsection{Introduction}

This chapter provides a simple preference axiomatization of cumulative prospect theory with a power utility function. Hopefully, this axiomatization will facilitate empirical investigations of cumulative prospect theory and, therefore, will make it better suited for theoretical studies.

Prospect theory (Kahneman \& Tversky 1979) was one of the first nonexpected utility models and remains today the most-used model in empirical studies and applications. In theoretical economic studies, however, prospect theory did not yet receive much attention, possibly because it lacked a well-founded method for transforming probabilities. Such a method was introduced by Quiggin (1981) in "rank-dependent utility." In theoretical studies, rank-dependent utility is currently the most-used nonexpected utility model.

The empirical features of prospect theory and the theoretical advantages of rank-

\footnotetext{
${ }^{0}$ The results in this chapter were first formulated in Wakker \&: Zank (1998b).
} 
dependent utility were combined in cumulative prospect theory (Tversky \& Kahneman 1992, Luce \& Fishburn 1991, Starmer \& Sugden 1989). The main feature added to rankdependent utility is a special role for a status quo outcome and thus a different treatment of gains and losses. That different treatment, in particular loss aversion (losses weigh more heavily than gains), is one of the main factors in human risk attitude. Much of the empirically observed risk aversion is due to loss aversion. Throughout the last decades, authors have emphasized the relevance of the status quo outcome in empirical decision making (Markowitz 1952, Arrow 1953, Edwards 1954, Yaari 1965, Kahneman \& Tversky 1979, Luce \& Fishburn 1991, Tversky \& Kahneman 1991, Tversky \& Kahneman 1992). One of the main paradoxes in economics, the equity premium puzzle, has been explained by loss aversion (Bateman et al. 1997, Gneezy \& Potters 1997).

Two theoretical foundations of cumulative prospect theory, by means of preference axioms, have as yet been published (Tversky \& Kahneman 1992, Wakker \& Tversky 1993). These axiomatizations are not easily accessible and hence cannot be easily used in experimental investigations or theoretical studies. This chapter presents a considerably simplified axiomatization. The simplification is achieved by the added assumption of power utility, i.e., constant proportional risk aversion. ${ }^{1}$ Constant proportional risk aversion has been a useful tool in classical studies of risk (Tversky 1967, Keeney \& Raiffa 1976, Wolf \& Pohlman 1983, Cass \& Stiglitz 1972) and has served as a benchmark condition in many empirical investigations (Cohn, Lewellen, Lease, \& Schlarbaum 1975, Friend \& Blume 1975, Binswanger 1981, Szpiro 1986, Levy 1994). It has also served as a useful tool in modern nonexpected utility theories (Holthausen 1981, Hogarth \& Einhorn 1990, Luce \& Fishburn 1991, Tversky \& Kahneman 1992, Kachelmeier \& Shehata 1992, Luce, Mellers, \& Chang 1993, Benartzi \& Thaler 1995, Tversky \& Fox 1995, Wu \& Gonzalez 1996, Fox \& Tversky 1996, Bernstein et al. 1997, Beetsma \& Schotman 1997, Donkers, Melenberg, \& van Soest 1998, Fennema \& van Assen 1997, Thaler, Tversky, Kahneman, \& Schwartz 1997, Safra \& Segal 1997). For applications to the measurement of inequality, see Atkinson (1970), Sen (1973), Ben-Porath, Gilboa, \& Schmeidler (1994, 1997), Chateauneuf (1996b), and Safra \& Segal (1997).

Tversky \& Kahneman (1992) tested constant proportional risk aversion nonparametrically, i.e., from binary preferences, and found it confirmed. The belonging power utility family performed well in parametric tests. For example, it was preferred to other parametric families for its better fit and higher stability in (Camerer \& Ho 1994, footnote 22). Also

\footnotetext{
${ }^{1}$ Also known as constant relative risk aversion and first characterized by Pratt (1964),
} 
Fishburn \& Kochenberger (1979), Krzysztofowicz \& Koch (1989), and Chechile \& Cooke (1997) found that the power family fits best. Psychological explanations for the prevalence of power perception functions have been provided by Stevens (1959) and by Fetherstonhaugh, Slovic, Johnson, \& Friedrich (1997). Because of these findings, the power model is generally assumed in parametric tests and provides the most-used nonexpected utility form nowadays.

When outcomes are only gains (or only losses), then cumulative prospect theory naturally reduces to rank-dependent utility. Thus, a characterization of rank-dependent utility with power utility (Corollary 4.4) follows as a corollary of the main result of this chapter (Theorem 4.2). It may seem surprising that the axiomatizations with constant proportional risk aversion included are so much simpler than without it. Indeed, under the common assumptions of weak ordering, continuity, and first stochastic dominance, the natural "comonotonic" weakening of von Neumanu-Morgenstern (1944) independence is then sufficient to axiomatize cumulative prospect theory. The separation of utility and decision weights thus comes by "free of charge." and does not need additional preference conditions.

The preference axiomatizations for rank-dependent utility and cumulative prospect theory, provided so far in the literature, did not incorporate constant proportional risk aversion. They therefore had to invoke additional preference conditions, more complex than comonotonic independence, to separate utilities fronn decision weights. Examples are Axiom 4 of Quiggin (1982), weak event commutativity (Chew 1989, Chew \& Karni 1994), joint receipt (Luce \& Fishburn 1991), comonotonic tradeoff consistency (Tversky \& Kahneman 1992, Wakker \& Tversky 1993, Wakker 1994), and weak multisymmetry (Nakamura 1990, 1992, see also Nakamura 1995, Axiom 5, and Gul 1992, Assumption 2). To date, one axiomatization has succeeded in using only preference conditions that directly weaken von Neumann-Morgenstern independence (Chateauneuf 1996). Its comonotonic mixture independence axiom is still more complex than comonotonic independence. The special case of rank-dependent utility with linear utility and a quadratic probability transformation is characterized in Safra \& Segal (1997, Theorem 3) by means of constant proportional risk aversion plus constant absolute risk aversion and some other axioms. An interesting aspect of that result is that it does not use the rank-ordering of outcomes as a primitive.

It is particularly remarkable that cumulative prospect theory, the sign-dependent generalization of rank-dependent utility, follows from the axioms presented here without requiring any further preference condition to induce sign dependence. That is, the different 
treatment of gains and losses follows again "free of charge." The explanation for this natural fit of cumulative prospect theory and constant proportional risk aversion lies in the special role of the status quo (zero) outcome in both.

It is remarkable that curnulative prospect theory, in spite of its empirical realism and theoretical soundness, has not yet received much attention in the economics literature (Wakker, 1998). By extending rank-dependent utility to incorporate loss aversion, which is important for empirical realism and applications, and by simplifying cumulative prospect theory so as to make it suited for theoretical analyses, increasing the interest of economists in curmulative prospect theory is intended.

\subsection{Definitions}

The set of outcomes is $I R$, the set of real numbers. A lottery $P=\left(p_{1}, x_{1} ; \ldots ; p_{n}, x_{n}\right)$ is a finite probability distribution over the set of outcomes, assigning probability $p_{j}$ to outcome $x_{j}, j=1, \ldots, n$. The probabilities $p_{j}$ are nonnegative and sum to one. Lotteries are written in a rank-ordered form, i.e., the above notation implicitly means that the outcomes are rank-ordered $\left(x_{1} \geqslant \cdots \geqslant x_{n}\right)$.

Positive outcomes are gains and negative outcomes are losses; the zero outcome is the status quo. A lottery $P$ can be decomposed into a gain-part $P^{+}$and a loss-part $P^{-}$, where $P^{+}$is the lottery $P$ with all the negative outcomes replaced by 0 and $P^{-}$the lottery with all positive outcomes replaced by 0 . Luce \& von Winterfeldt (1994) argued for the psychological plausibility of the separation into a gain- and loss-part. Fishburn (1977) and Holthausen (1981) interpreted $P^{-}$as the risk part and $P^{+}$as the return part of the lottery, using a "target outcome" in the role of status quo.

We assume a preference relation $\succcurlyeq$ over lotteries; the symbols $\succ, \sim, \preccurlyeq$ and $\prec$ are defined as usual. $V$ is a representing function or representation for $\succcurlyeq$ if $V$ maps lotteries to the reals such that $P \succcurlyeq Q \Leftrightarrow V(P) \geqslant V(Q)$. If a representing function exists then $\succcurlyeq$ is a weak order, i.e., it is complete $(P \succcurlyeq Q$ or $P \preccurlyeq Q$ for all lotteries $P, Q)$ and transitive.

Rank-dependent utility $(R D U)$ holds if a representation exists of the form

$$
\left(p_{1}, x_{1} ; \ldots ; p_{n}, x_{n}\right) \mapsto \sum_{j=1}^{n} \pi_{j} U\left(x_{j}\right),
$$

explained next. $U$, the utility function, is a function from the outcomes to the reals. The utility functions considered in this chapter will all be continuous and strictly increasing. 
The decision weights are defined as

$$
\pi_{j}=w\left(p_{1}+\cdots+p_{j}\right)-w\left(p_{1}+\cdots+p_{j-1}\right)
$$

where $w$ is a probability transformation, i.e., it is strictly increasing from $[0,1]$ to $[0,1]$ and satisfies $w(0)=0$ and $w(1)=1$. Continuity of $w$ is left optional in all theorems, hence is not imposed in the definition.

Cumulative prospect theory (CPT) holds if the preference relation $\succcurlyeq$ can be represented by a CPT functional, defined next. Consider a lottery $P=\left(p_{1}, x_{1} ; \ldots ; p_{n}, x_{n}\right)$ and let

$$
x_{1} \geqslant \cdots \geqslant x_{k} \geqslant 0>x_{k+1} \geqslant \cdots \geqslant x_{n},
$$

for some $k \in\{0, \ldots, n\}$. Then the CPT value of the lottery is again given by formula (4.1), with the following modifications. $U$ is again the utility function, but now

$$
U(0)=0
$$

is set. For the decision weights, we assume a probability transformation $w^{\frac{4}{7}}$ for gains and another one, $w^{-}$, for losses. For $j \leqslant k$ (gains), $\pi_{j}$ is defined as in rank-dependent utility with respect to $w^{+}$, i.e.,

$$
\pi_{j}=w^{+}\left(p_{1}+\cdots+p_{j}\right)-w^{+}\left(p_{1}+\cdots+p_{j-1}\right) .
$$

For $j>k$ (losses), $\pi_{j}$ is derived from $w^{-}$in a dual manner, i.e.,

$$
\pi_{j}=w^{-}\left(p_{j}+\cdots+p_{n}\right)-w^{-}\left(p_{j+1}+\cdots+p_{n}\right) .
$$

Thus, CPT can be written as the RDU value of the gain-part of the lottery with respect to a probability transformation $u$ " plus a "dual," obviously negative, RDU value of its loss-part with respect to a probability transformation $w^{-}$. The latter is defined in a dual manner by means of a "bottom-up" integration which would coincide with the regular RDU value if the dual of $w^{-}(p)$, defined by $1-w^{-}(1-p)$, were used instead of $w^{-}$.

Let us summarize. The CPT-formula is

$$
\begin{aligned}
\left(p_{1}, x_{1} ; \ldots ; p_{n}, x_{n}\right) \mapsto & \sum_{i=1}^{k}\left[w^{+}\left(p_{1}+\cdots+p_{i}\right)-w^{+}\left(p_{1}+\cdots+p_{i-1}\right)\right] U\left(x_{i}\right)+ \\
& \sum_{j=k+1}^{n}\left[w^{-}\left(p_{j}+\cdots+p_{n}\right)-w^{-}\left(p_{j+1}+\cdots+p_{n}\right)\right] U\left(x_{j}\right) .
\end{aligned}
$$


Here

$$
\mathrm{CPT}(P)=\mathrm{CPT}\left(P^{+}\right)+\mathrm{CPT}\left(P^{-}\right)
$$

where both terms on the right-hand side are regular RDU forms.

In general expected utility models with $\mathbb{I} R_{++}$as outcome set, constant proportional risk aversion can generate any utility function from the "log/power" family. In the present model, however, utility must also be defined at zero, hence only the "positive power" functions remain possible. A function $U: \mathbb{R} \rightarrow \mathbb{R}$ is from the positive power family for gains if

$$
U(x)=\sigma^{+} \cdot x^{\alpha}, \text { with } \sigma^{+}>0, \alpha>0, \text { for all } x \geqslant 0,
$$

and it is from the positive power family for losses if

$$
U(x)=-\sigma^{-} \cdot|x|^{\beta}, \text { with } \sigma^{-}>0, \beta>0, \text { for all } x \leqslant 0 .
$$

The scale factors $\sigma^{+}$and $\sigma^{-}$are positive so as to guarantee strict increasingness. We will require $U(0)=0$, hence no location parameters have been added.

\subsection{A Preference Characterization}

The central property in this chapter is constant proportional risk aversion. It is imposed when all outcomes are of the same sign and then requires invariance of preference with respect to outcome-multiplication by a common positive factor. That is, for all positive numbers $\mu$,

$\left(p_{1}, x_{1} ; \ldots ; p_{n}, x_{n}\right) \succcurlyeq\left(p_{1}, y_{1} ; \ldots ; p_{n}, y_{n}\right) \Rightarrow\left(p_{1}, \mu \cdot x_{1} ; \ldots ; p_{n}, \mu \cdot x_{n}\right) \succcurlyeq\left(p_{1}, \mu \cdot y_{1} ; \ldots ; p_{n}, \mu \cdot y_{n}\right)$

whenever either all outcomes are gains or all outcomes are losses. Constant proportional risk aversion is necessary for CPT with positive power utility, because then the CPT functional is homogeneous of degree $\alpha$ for gain-lotteries and of degree $\beta$ for loss-lotteries.

The next preference condition weakens von Neumann \& Morgenstern independence by bringing in a comonotonicity restriction. In fact, it weakens independence somewhat further, by only considering maximal or minimal common outcomes. As a preparation, the probabilistic mixing of lotteries is defined. Given two lotteries $P=\left(p_{1}, x_{1} ; \ldots ; p_{n}, x_{n}\right), Q=$ $\left(q_{1}, y_{1} ; \ldots ; q_{m}, y_{m}\right)$, and $\gamma \in[0,1]$, the mixture $\gamma P+(1-\gamma) Q$ is the lottery ${ }^{2}$

$$
\left(\gamma p_{1}, x_{1} ; \ldots ; \gamma p_{n}, x_{n} ;(1-\gamma) q_{1}, y_{1} ; \ldots ;(1-\gamma) q_{m}, y_{m}\right) .
$$

\footnotetext{
${ }^{2}$ For simplicity, we have not permuted the outcomes as would be required to maintain the rank-ordered notation of lotteries.
} 
The preference relation satisfies tail independence if for all lotteries $P, Q, C, C^{\prime \prime}$ and all $\gamma \in(0,1)$ the following holds:

$$
\begin{aligned}
\gamma P+(1-\gamma) C & \succcurlyeq \gamma Q+(1-\gamma) C \\
& \Leftrightarrow \\
\gamma P+(1-\gamma) C^{\prime} & \succcurlyeq \gamma Q+(1-\gamma) C^{\prime},
\end{aligned}
$$

whenever either all outcomes in the lotteries $C, C^{\prime}$ are above those of $P, Q$, or they are all below.

The condition, extended to nonsimple lotteries, was introduced by Green \& Jullien (1988), who called it ordinal independence. Von Neumann \& Morgenstern independence is more restrictive because it, first, does not impose restrictions on the outcomes of $P, Q, C, C^{\prime}$, and, second, it requires that the preferences should also agree with those between the unmixed $P$ and $Q$. Tail independence is implied by CPT, i.e., it is not affected by rank and sign dependence. The proof is provided in the mairı text because it may clarify the nature of the CPT form.

\section{LEMma 4.1 CPT implies tail independence.}

Proof. We discuss the following formula for $s=+$ and $s=-$, and with $s$ dropped. Note that the $s$ superscript and mixing are compatible, e.g., $(\gamma P+(1-\gamma) C)^{s}=\gamma P^{s}+(1-\gamma) C^{s}$.

$$
\begin{aligned}
C P T\left(\gamma P^{s}+(1-\gamma) C^{s}\right) & -C P T\left(\gamma Q^{s}+(1-\gamma) C^{s}\right) \\
& = \\
C P T\left(\gamma P^{s}+(1-\gamma) C^{\prime s}\right) & -C P T\left(\gamma Q^{s}+\left(1-\gamma^{\prime}\right) C^{\prime s}\right) .
\end{aligned}
$$

Both for $s=+$ and for $s=-$, the outcomes of $C^{s}$ and $C^{4 s}$ are all rank-ordered above those of $P^{s}$ and $Q^{s}$ or below, as they are without the superscript $s$. The above formula holds true for $s=+$ and $s=-$, CPT being an RDU form in both cases. (The $(1-\gamma) C^{s}$ and $(1-\gamma) C^{\prime s}$ parts of the RDU values cancel in both differences.) By summation (Formula 4.4), the formula also holds true if the superscript $s$ is dropped. That implies, in particular, that the sign of the left-hand side is the same as of the right-hand side, from which tail independence follows.

$\succcurlyeq$ satisfies stochastic dominance if $\left(p_{1}, x_{1} ; \ldots ; p_{n}, x_{n}\right) \succ\left(p_{1}, y_{1} ; \ldots ; p_{n}, \hat{y}_{n}\right)$ whenever $x_{j} \geqslant y_{j}$ for all $j$ and $x_{j}>y_{j}$ for at least one $j$ with $p_{j}>0$. On the domain of simple 
lotteries, the formulation used here, reminiscent of outcome monotonicity, is equivalent to other common formulations in terms of distribution functions.

The continuity condition used here only relates to variations in outcomes and imposes the common Euclidean continuity of $I R^{n}$ on $n$-outcome lotteries, for all $n$. That is, $\succcurlyeq$ satisfies simple-continuity if, for any lottery $\left(p_{1}, x_{1} ; \ldots ; p_{n}, x_{n}\right)$, the sets

$$
\left\{\left(y_{1}, \ldots, y_{n}\right):\left(p_{1}, y_{1} ; \ldots ; p_{n}, y_{n}\right) \succcurlyeq\left(p_{1}, x_{1} ; \ldots ; p_{n}, x_{n}\right)\right\}
$$

and

$$
\left\{\left(y_{1}, \ldots, y_{n}\right):\left(p_{1}, y_{1} ; \ldots ; p_{n}, y_{n}\right) \preccurlyeq\left(p_{1}, x_{1} ; \ldots ; p_{n}, x_{n}\right)\right\}
$$

are closed subsets of $\mathbb{R}^{n}$. Continuity with respect to variation in probability is not required; it will be characterized in Observation 4.3 .

THEOREM 4.2 For the preference relation $\succcurlyeq$ on the set of lotteries over $I R$, the following two statements are equivalent:

(i) CPT holds, with a positive power utility for gains and a, possibly different, positive power utility for losses.

(ii) The preference relation $\succcurlyeq$ satisfies the following conditions:

(1) weak ordering,

(2) stochastic dominance,

(3) simple continuity,

(4) tail independence,

(5) constant proportional risk aversion for gains and for losses.

If (i) holds then the probability transformations are uniquely determined and utility is a ratio scale, i.e., it is unigue up to a positive scale factor.

Let us next discuss uniqueness of utility in more detail and define loss aversion. Suppose that the gains utility function is $\sigma^{+} x^{\alpha}$ and the loss utility function is $-\sigma^{-}|x|^{\beta}$; for positive $\sigma^{+}, \sigma^{-}$. We can freely multiply utility by a positive factor, which however must be the same for gains and losses. Hence we could choose any utility $\mu \sigma^{+} x^{\alpha}$ for gains and $-\mu \sigma^{-}|x|^{\beta}$ for losses. In particular, we can take $\mu$ equal to $1 / \sigma^{+}$. Defining $\lambda=\sigma^{-} / \sigma^{+}$we then get: $U(x)=x^{\alpha}$ for gains;

$U(0)=0$

$U(x)=-\lambda|x|^{\beta}$ for losses. 
The scaling of utility just described has been generally adopted in parametric studies of CPT. It means setting $U(1)=1$ and scaling loss aversion as $\lambda=-U(-1) / U(1)$. The scaling was used by Fishburn (1977) and Holthausen (1981) within the expected utility framework and by Luce \& Fishburn (1991, Equation 4) in their axiomatic derivation of CPT. Empirical estimations have usually found high values of $\lambda$ ("loss aversion"), typically exceeding 2. Tversky \& Kahneman (1992) found $\lambda=2.25$; see also Holthausen (1981) and the references therein, and Bernstein et al. (1997). These degrees of loss aversion have been used in new explanations of the equity premium puzzle (Benartzi \& Thaler 1995, Thaler, Tversky, Kahneman, \& Schwartz 1997, Gneezy \& Potters 1997).

Next continuity of the probability transformations is characterized. $\succcurlyeq$ satisfies continuity in probabilities for gains on $] 0,1[$ if, for all probabilities $p$ and outcomes $X>x>0$ :

If $(p, X ; 1-p, 0) \succ(1, x)$ then there exists $q<p$ such that still $(q, X ; 1-q, 0) \succ(1, x)$,

and the same holds with the two preferences and the inequality reversed, i.e.,

$$
\text { If }(p, X ; 1-p, 0) \prec(1, x) \text { then there exists } q>p \text { such that still }(q, X ; 1-q, 0) \prec(1, x) \text {. }
$$

$\succcurlyeq$ satisfies continuity in probabilities for gains at 1 if (4.6) holds for $p=1$; $\succcurlyeq$ satisfies continuity in probabilities for gains al 0 if (4.7) holds for $p=0$. Similar conditions can be defined for losses instead of gains by assuming that $X<x<0$ in (4.6) and (4.7) and reversing the strict preferences. The next result is similar to Wakker (1994, Theorem 12) for RDU.

ObServation 4.3 Assume that Statement (i) in Theorem 4.2 holds. Then continuity conditions of the probability transformations agree with the related continuity conditions of $\succcurlyeq$ in probability, with $w^{+}$related to gains preference conditions and $w^{-}$to loss preference conditions.

In the literature on decision under risk weak continuity is usually invoked, requiring not only continuity in outcomes, as above, but also continuity in probability. There is, however, empirical and theoretical interest in discontinuities of probability transformation at 0 and 1 (Bell 1982, Cohen \& Jaffray 1988, Prelec 1998).

Hence, the above more general result is presented. Next, the characterization of rankdependent utility is displayed as a corollary. The result is obtained by restricting the above analysis to nonnegative outcomes. 
COROLLARY 4.4 For the preference relation $\succcurlyeq$ on the set of lotteries over $\mathbb{I} R_{+}$, the following two statements are equivalent:

(i) RDU holds, with a positive power utility.

(ii) $\succcurlyeq$ satisfies the following conditions:

(1) weak ordering,

(2) stochastic dominance,

(3) simple continuity,

(4) tail independence,

(5) constant proportional risk aversion.

\subsection{Conclusion}

Given the large impact of loss aversion on human decision making, the sign-dependent generalization of rank-dependent utility, advanced by Tversky \& Kahneman (1992) and others, seems worthwhile. It has been used successfully in many empirical investigations, but has not yet been studied extensively in theoretical analyses. The latter may be due to its analytical complexity. The present chapter has presented a simplified analysis. It is hoped that the simplification will make the model more accessible, and facilitate its theoretical and empirical applications.

\subsection{Appendix: Proofs to Chapter 4}

First an auxiliary notation is introduced. For $n \in \mathbb{N}$ and a probability tuple $\left(p_{1}, \ldots, p_{n}\right)$, $L\left(p_{1}, \ldots, p_{n}\right)$ denotes the set of all lotteries of the form $\left(p_{1}, x_{1} ; \ldots ; p_{n}, x_{n}\right)$. Recall that it is implicitly understood in this notation that $x_{1} \geqslant \cdots \geqslant x_{n}$ and that the probabilities $p_{3}$ are nonnegative and sum to one.

LEMMA 4.5 For each finite number of sets $L\left(q_{1}^{1}, \ldots, q_{n_{1}}^{1}\right), \ldots, L\left(q_{1}^{m}, \ldots, q_{n_{m}}^{m}\right)$, there is one set $L\left(p_{1}, \ldots, p_{n}\right)$ that contains them all. In particular, for each finite set of lotteries, there is a set $L\left(p_{1}, \ldots, p_{n}\right)$ that contains them all.

Proof. As an example, $L(1 / 2,1 / 2)$ and $L(1 / 3,2 / 3)$ are contained in $L(1 / 3,1 / 6,1 / 2)$. For the general case, define the set of "cumulative probabilities," $q_{i}^{j \prime}=q_{1}^{j}+\cdots+q_{i}^{j}$ for all $i, j$, 
take the set of all such $q_{i}^{i \prime}$, and rank-order them from lowest to highest. The differentials between these levels of cumulative probabilities are the $p_{j} \mathrm{~s}$.

Proof of THeorem 4.2.First Statement (i) is assumed and (ii) is derived. Weak ordering is immediate and stochastic dominance follows because both utility and the probability transformations are strictly increasing. Simple continuity follows from continuity of utility. Tail independence holds because of Lemma 4.1 and constant proportional risk aversion because of homogeneity of the CPT functional for gains and losses. (ii) has been established.

In the rest of this proof, Statement (ii) is assumed and Statement (i), as well as the uniqueness results, are derived. In the first lemmas, up to Corollary 4.9 , a CPT representation is derived on a fixed set $L\left(p_{1}, \ldots, p_{n}\right)$. This being a subset of the lottery domain, the representation is, formally speaking, a restriction of a CPT representation. Similarly, the probability transformation functions, only being defined on a finite number of probabilities within the unit interval, are then restrictions of probability transformations. At the end of the proof, the representation and the belonging functions are extended to the whole domain.

LEMMA 4.6 Whenever $n \geqslant 3$, and $p_{1}, \ldots, p_{n}$ are positive, there exist continuous strictly increasing functions $V_{1}, \ldots, V_{n}$ such that $V_{j}(0)=0$ for all $j, \sum_{j=1}^{n} V_{j}(1)=1$, and

$$
\left(p_{1}, x_{1} ; \ldots ; p_{n}, x_{n}\right) \mapsto \sum_{j=1}^{n} V_{j}\left(x_{j}\right)
$$

represints $\succcurlyeq$ on $L\left(p_{1}, \ldots, p_{n}\right)$. The $V_{j}$ s are uniquely determined.

Proof. Define $I R_{\mathrm{i}}^{n}=\left\{x \in \mathbb{R}^{n}: x_{1} \geqslant \cdots \geqslant x_{n}\right\}$. It is identified with $L\left(p_{1}, \ldots, p_{n}\right)$ in the obvious manner. Thus the preference relation $\succcurlyeq$ on $L\left(p_{1}, \ldots, p_{n}\right)$ induces one, denoted by $\succcurlyeq^{\prime}$, on $\mathbb{R}_{l}^{n}$. $\succcurlyeq^{\prime}$ is a continous weak order that is monotonic, i.e., $x \succ^{\prime} y$ whenever $x_{j} \geqslant y_{j}$ for all $j$ and $x_{j}>y$, for at least one $j$. The latter follows from positiveness of the probabilities and stochastic dominance.

Tail independence of $\succcurlyeq$ implies tail independence of $\succcurlyeq^{\prime}$, that is, if two elements of $I R_{!}^{n}$ have the first $i$ or the last $j$ coordinates in common, then the preference between them is independent of those common coordinates. To explain this, we compare the preference between $\left(p_{1}, x_{1} ; \ldots ; p_{m}, x_{m} ; p_{m+1}, c_{m+1} ; \ldots ; p_{n}, c_{n}\right)$ and $\left(p_{1}, y_{1} ; \ldots ; p_{m}, y_{m} ; p_{m+1}, c_{m+1} ; \ldots ; p_{n}, c_{n}\right)$ 
with the preference between

$\left(p_{1}, x_{1} ; \ldots ; p_{m}, x_{m} ; p_{m+1}, c_{m+1}^{\prime} ; \ldots ; p_{n}, c_{n}^{\prime}\right)$ and $\left(p_{1}, y_{1} ; \ldots ; p_{m}, y_{m} ; p_{m+1}, c_{m+1}^{\prime} ; \ldots ; p_{n}, c_{n}^{\prime}\right)$.

Define $\sum_{j=1}^{m} p_{j}=\gamma, P=\left(\frac{p_{1}}{\gamma}, x_{1} ; \ldots ; \frac{p_{m}}{\gamma}, x_{m}\right), Q=\left(\frac{p_{1}}{\gamma}, y_{1} ; \ldots ; \frac{p_{m}}{\gamma}, y_{n}\right), \sum_{j=m+1}^{n} p_{j}=1-\gamma$, $C=\left(\frac{p_{m+1}}{1-\gamma}, c_{m+1} ; \ldots ; \frac{p_{n}}{1-\gamma}, c_{n}\right)$, and $C^{\prime}=\left(\frac{p_{m+1}}{1-\gamma}, c_{m+1}^{\prime} ; \ldots ; \frac{p_{n}}{1-\gamma}, c_{n}^{\prime}\right)$. Then tail independence of $\succcurlyeq$ implies that the two considered preferences are the same, i.e., tail independence holds for $\succcurlyeq^{\prime}$.

Now the existence of the $V_{j}$ functions follows from a generalization of Theorem 3.2 of Wakker (1993b) as described by Chateauneuf \& Wakker (1993). The details of the proof, unfortunately, have to combine several results from different papers and are described briefly here. For $n \geqslant 3$ the condition "CI" of Wakker (1993b) (complete independence of preferences from common coordinates, also called (con)joint independence or strong separability or the sure-thing principle in the literature) has been weakened here to tail independence. That additive representability, i.e, existence of functions $V_{j}$ as in the lemma, then still holds on the "rank-ordered" set $\mathbb{R}_{\downarrow}^{n}$, follows from Corollary C.5 of Chateauneuf \& Wakker (1993) and Gorman (1968): In Gorman's terminology, separability of $\{1, \ldots, i\}$ and $\{i, \ldots, n\}$ implies, within each box within $\mathbb{I}_{\downarrow}^{n}$, separability of $\{1, \ldots, i-1, i+1, \ldots, n\}$. This implies that every preference is independent of any single common coordinate, i.e., Wakker's (1993b) CI holds within each box. This implies, by Gorman (1968) or Debreu (1960), additive representability on each box within $I R_{!}^{n}$, i.e., "Iocal" additive representability. From that, global additive representability follows on our rank-ordered domain by Theorem 2 and Chateauneuf \& Wakker (1993, Lemma C.5), and the absence of maximal and minimal elements.

The $V_{j}$ s derived in the literature are usually unique up to a location and a common scale. Our choices of their locations and scale uniquely determine the functions.

LEMMA 4.7 For the lotteries in $L\left(p_{1}, \ldots, p_{n}\right)$ with nonnegative outcomes, the representation in Lemma 4.6 is the restriction of a CPT form, with $U$ from the positive power family and $U(0)=0, U(1)=1 . U$ is unique and so is the (restriction of a) probability transformation function $w^{+}$on its domain which consists of all values $\sum_{j=1}^{i} p_{j}, i=1, \ldots, n$; there $w^{+}$is strictly increasing.

Proof. Let $V_{1}, \ldots, V_{n}$ be as in Lemma 4.6. We define $\succcurlyeq^{\prime}$ on $\mathbb{I R}_{1}^{n}$ as in the proof of Lemma 4.6 and restrict attention to lotteries with nonnegative outcomes. If the representation in Lemma 4.6 is a CPT form, then necessarily $U(\mu)=\sum_{j=1}^{n} V_{j}(\mu)$ for all nonnegative $\mu$. Hence we have to define $U$ in that manner and derive CPT for that already uniquely determined utility function. Note that $U(0)=0$ and $U(1)=1$. 
Fix some $1 \leqslant m<n$. We restrict attention to $n$-tuples of nonnegative outcomes for which the first $m$ coordinates are identical, and so are the last $n-m$ ones, and write them as $\left(x_{1, m}, x_{m+1, n}\right)$ for $x_{1, m} \geqslant x_{m+1, n} \geqslant 0 . \nvdash^{\prime}$ is represented on this two-dimensional subset of $n$-tuples by $V_{1, m}\left(x_{1, m}\right)+V_{m+1, n}\left(x_{m+1, n}\right)$ where $V_{1, m}\left(x_{1, m}\right)=\sum_{j=1}^{m} V_{j}\left(x_{1, m}\right)$ and $V_{m+1, n}\left(x_{m+1, n}\right)=\sum_{j=m+1}^{n} V_{j}\left(x_{m+1, n}\right)$. Because of constant proportional risk aversion, $\xi^{\prime}$ is invariant on this subset of lotteries under positive scalar-multiplication of outcomes. Miyamoto \& Wakker (1996, Theorem 2) consider only gains $(>0)$ and show that $V_{1, m}$ and $V_{m+1, n}$ are proportional when restricted to gains. Obviously, they also are proportional when the zero outcome is included. $V_{1, m}$ and $V_{m+1, n}$ must be proportional to their sum $V_{1, m}+V_{m+1, n}=U$, hence they are of the form

$$
V_{1, m}=\pi_{1, m} U \text { and } V_{m+1, n}=\pi_{m+1, n} U,
$$

for positive uniquely determined $\pi_{1, m}$ and $\pi_{m+1 . n}$ that sum to one. Theorem 2 of Miyamoto \& Wakker (1996) also implies that for gains $U$ is either from the positive power family, or logarithmic, or from the negative power family; the latter is defined as in (4.5) but with $\alpha$ and $\sigma$ negative. The logarithmic family and the negative power family are excluded in our case because strict increasingness (or continuity) at zero would then imply that $V_{1, m}(0)=-\infty$ which is excluded because $V_{1, m}(0)=0$. We conclude that

$U$ is a positive power function for nonnegative outcomes.

Define $\pi_{1}=\pi_{1,1}, \pi_{j}=\pi_{1, j}-\pi_{1, j-1}$ for $j=2, \ldots, n-1$, and $\pi_{n}=\pi_{n, n}(=1-$ $\pi_{1, n, \cdots 1}$ ). By definition, $V_{1,1}=V_{1}=\pi_{1} U$. Formula (4.9) now implies, inductively, that $V_{j}=V_{1, j}-V_{1, j-1}=\pi_{j} U$ for $j=2, \ldots, n-1$, and then also $V_{j}=\pi_{j} U$ for $j=n$. This and monotonicity imply that all $\pi_{j} s$ are positive. They sum to one. We define a (restriction of a) probability transformation function $w^{+}$so as to properly transform cumulative probabilities into cumulative decision weights; that is, $w^{+}\left(\sum_{i=1}^{j} p_{i}\right)=\sum_{i=1}^{j} \pi_{i}$. Note that $w^{+}$is strictly increasing on its domain.

It follows that the representation of Lemma 4.6 is the restriction of a CPT form with respect to $w^{+}$and $U$ on the set of lotteries in $L\left(p_{1}, \ldots, p_{n}\right)$ with nonnegative outcomes. Uniqueness of $U$ and the $\pi_{j}$ s was pointed out when they were defined.

LEMMA 4.8 For the lotteries in $L\left(p_{1}, \ldots, p_{n}\right)$ with nonpositive outcomes, the representition in Lemma 4.6 is the restriction of a CPT form, with $U$ from the positive power family and $U(0)=0 . U$ is unique and so is the (restriction of a) probability transformation 
function $w^{--}$on its domain which consists of the values $\sum_{j=i}^{n} p_{j}, i=1, \ldots, n$; there $w^{-}$is strictly increasing.

Proof. Now we only consider lotteries with nonpositive outcomes. It can be demonstrated by a reasoning, similar to the proof of Lemma 4.7, that the representation of Lemma 4.6 is again a restriction of a CPT form. To preserve the scaling convention $\sum_{j=1}^{n} V_{j}(1)=$ $1=U(1)$, we have no more liberty to choose the scale of utility for losses. It is uniquely determined by $U(-1)=\sum_{j=1}^{n} V_{j}(-1)$ (which was defined as the loss aversion parameter $\lambda$ in the main text). Other than that, the reasoning for nonnegative outcomes can entirely be repeated. $U$ is also a positive power function for losses, but may obviously have a different exponent than for gains. The decision weights for losses are determined by $w^{-}\left(p_{n}\right)$, $w^{-}\left(p_{n}+p_{n-1}\right)-w^{-}\left(p_{n}\right)$, etc.

COROLLARY 4.9 The representation in Lemma 4.6 is (the restriction of) a CPT representation with $U$ from the positive power family for gains and also for losses, and $U(0)=0$ and $U(1)=1 . U$ is unique and so is the (restriction of) the probability transformation function $w^{+}$at the values $\sum_{j=1}^{i} p_{j}, i=1, \ldots, n$ and $w^{-}$at the values $\sum_{j=i}^{n} p_{j}, i=1, \ldots, n$.

Proof. That the representation of Lemma 4.6 is a CPT form for nonnegative outcomes, and also for nonpositive outcomes, was demonstrated in the preceding lemmas. Next consider a "mixed" lottery $P$ (one with both gain and loss outcomes). The additive representation of Lemma 4.6 is a sum of its value at $P^{+}$and at $P^{-}$. Hence the value of the mixed lottery $P$ is $\mathrm{CPT}\left(P^{+}\right)+\mathrm{CPT}\left(P^{-}\right)$. By Formula (4.4), CPT follows.

Next the CPT representation is extended to the set of all simple lotteries. The CPT representations on two different sets of lotteries $L\left(p_{1}, \ldots, p_{n}\right)$ and $L\left(q_{1}, \ldots, q_{m}\right)$ coincide in the sense that the utility function $U$ is the same for both sets and $w^{+}$and $w^{-}$agree on common domain points: That follows from considering any set $L\left(r_{1}, \ldots, r_{k}\right)$ that contains both sets of lotteries; the latter set always exists by Lemma 4.5. By considering all sets $L\left(p_{1}, \ldots, p_{n}\right), w^{+}$and $w^{-}$are determined on the entire unit interval $[0,1]$. They are strictly increasing by Lemmas 4.7 and 4.8 .

We have now obtained one utility function $U_{2}$ and probability transformation functions $w^{+}$and $w^{-}$defined on the entire unit interval $[0,1]$, such that for every pair of lotteries their CPT value is determined. Every pair of lotteries is contained in some set $L\left(p_{1}, \ldots, p_{n}\right)$, on this set CPT represents preference, hence CPT represents preference between every pair of lotteries. 
We finally turn to uniqueness of the representation. For any other CPT representation with $U^{*}, w^{+*}, w^{-*}$, reconsideration of the above analysis shows that $U^{*}(\mu) / U^{*}(1)$ must agree with $U$, and next that the two probability transformations $w^{+*}$ and $w^{-*}$ must agree with $w^{+}$and $w^{-}$. Conversely, for any positive $\tau, U$ can be replaced by $\tau U$. This completes the proof of Theorem 4.2 .

ProOF of Observation 4.3. The range of utility is an interval with 0 in its interior. Hence the following facts follow from substitution and the fact that all probability transformation functions are strictly increasing: Formula (4.6) implies that $w^{+}$cannot "jump down" to the left of $p$, hence is left continuous at $p$. Formula (4.7) implies that $w^{+}$cannot "jump up" to the right of $p$, hence is right continuous at $p$. Similar facts hold for $w^{-}$and losses.

Proof of Corollary 4.4. This follows from restricting the proof of Theorem 4.2 to nonnegative outcomes. In particular logarithmic and negative power utility is still excluded because the zero outcome is present. If outcomes would have been restricted to $\mathbb{R} R_{++}$, utility could also have been logarithmic or a negative power. 



\section{Chapter 5}

\section{Cumulative Prospect Theory for Parametric and Multiattribute Utilities}

\subsection{Introduction}

Based on empirical grounds cumulative prospect theory (CPT) supports the distinction of outcomes into gains and losses. The cumulative probabilities are transformed according to this separation, and the utility function also reflects that distinction. Kahneman \& Tversky (1979) and Tversky \& Kahneman (1992) proposed a utility function which is concave for gains, convex for losses, and steeper for losses than for gains. From their experiments, in which lotteries on monetary outcomes were valued, Tversky \& Kahneman (1992) concluded that a two-sided power function is a good approximate for the utility function. Different powers for gains than for losses were deduced.

The empirical analysis of Currim and Sarin (1989) confirms the properties of utility and of the weighting functions in the curnulative prospect theory model. They fitted an exponential form for utility, and found evidence for different decision weights for gains than for losses. Smidts (1997) concluded from his data, that an exponential utility fits better than a power utility. In a different experimental study Beetsma and Schotman (1998) conclude that the exponential and the power utility perforn equally well.

For decision under risk a complete axiomatization of cumulative prospect theory with

\footnotetext{
${ }^{6}$ The results in this chapter were first formulated in Zank (1998).
} 
a two-part power utility is presented in Wakker \& Zank (1998b), (see also Chapter 4). There, constant proportional risk aversion of the preference relation determines the nature of utility, in the presence of the simple axioms: weak ordering, continuity, stochastic dominance and tail independence, the latter being a weakening of the independence condition of von Neumann \& Morgenstern (1944).

However, as mentioned above, interest in a special form for utility is not limited to constant proportional risk aversion. Linear/exponential, additive/multiplicative or multilinear utility families are also topic for many analytic studies (Currim \& Sarin, 1989; Smidts 1997; Beetsma \& Schotman, 1998). Miyamoto \& Wakker (1996) derived models characterizing such families of utilities, assuming the additive representation for a preference relation on rank-ordered acts given beforehand. They point out that, when there is interest in a specific form of utility, preference axioms need not immediately imply a separation of probabilities and utilities in the representing function, but can be weakened to imply only additive representability. Then, in the presence of constant proportional (absolute) risk aversion or utility independence, probabilities and utilities can be identified. This feature will be captured in all our models. First additive representability is established, and from that a cumulative prospect theory functional is derived.

Other parametric families of utilities, focusing on decreasing or increasing risk aversion, were characterized by Farquhar \& Nakamura (1987) and Bell (1988), the so called "polynomial-exponential" utility functions, a family including the "sumex" utilities pre sented in Nakamura (1996). Saha (1993) proposed the "expo-power" utility, a form which exhibits decreasing or increasing absolute risk aversion and decreasing or increasing proportional risk aversion, depending on the values of the parameters involved. However, such families will not be discussed here.

This chapter deals with decision under uncertainty. For a finite set of states, we first derive a CPT-model with linear or exponential utility. Here, the central property of the preference relation is constant absolute risk aversion. This, in addition to weak ordering, monotonicity, continuity and tail independence, constitutes necessary and sufficient axioms for the derivation of such a model.

Secondly, we concentrate on a CPT-model with utility as a power function, where constant proportional risk averse preferences are considered.

Thirdly, for multiattribute outcomes, we focus on preferences satisfying mutual utility independence or utility independence for attributes, deriving CPT-models with additive/multiplicative or multilinear utility, respectively. Also here the additional axioms 
are weak ordering, monotonicity, continuity and tail independence, simple axioms, which imply the existence of additive representing functions on rank-ordered sets.

Proofs are presented in the Appendix, except for Lemma 5.1.

\subsection{Cumulative Prospect Theory with Linear/Exponential or Power Utility}

Throughout this chapter $S=\{1, \ldots, n\}$, with $n \geqslant 3$, is a finite set of states, where exactly one state is true and there is uncertainty about which is the true state; subsets of $S$ are events. An act $f$ assigns to each state $j \in S$ an outcome $f(j)$, or $f_{j}$ for short. In this section the set of outcomes is the set of real numbers $R$, and thus, we view the set of acts as the Cartesian product $\mathbb{R}^{n}$. Pasitive outcomes are gains and negative outcomes are losses; they are separated by the zero outcome which is the status quo. Hence, an act $f$ consists of a gain-part $f^{+}$and a loss-part $f^{-}$, where $f^{+}$is the act $f$ with all losses replaced by the status quo, and $f^{-}$is the act $f$ with all gains replaced by the status quo. Sometimes we identify the constant act $(x, \ldots, x) \in \mathbb{R}^{n}$ with the outcome $x \in \mathbb{R}$.

An act $f$ is rank-ordered if its outcomes are ordered as follows: $f_{1} \geqslant \cdots \geqslant f_{n}$. For each act there exists a permutation $\rho$ of $\{1, \ldots, n\}$ such that $f_{\rho(1)} \geqslant \cdots \geqslant f_{\rho(n)}$, i.e. the outcomes are rank-ordered with respect to $\rho$. For each permutation $\rho$ of $\{1, \ldots, n\}$ the set $\mathbb{R}_{\rho}^{n}$ consists of those acts which are rank-ordered according to $\rho$. For example, if $\rho=i d$ (i.e. $\rho(i)=i$ for all $i$ ), then $\mathbb{R}_{i d}^{n}$ is the set of rank-ordered acts.

On the set of acts we assume a preference relation denoted by $\succcurlyeq$. The symbols $\succcurlyeq, \succ$ $, \sim, \preccurlyeq, \prec$, are defined in the usual way, i.e. $f \succ g$ means $[f \geqslant g$ and not $g \succcurlyeq f], f \succ g$ means $[f \succcurlyeq g$ and $g \succcurlyeq f], f \preccurlyeq g$ means $g \succcurlyeq f$, and $f \prec g$ means $g \succ f$.

$V$ is a representing function or representation for $\succcurlyeq$ if $V$ assigns to each act a real value such that $f \succcurlyeq g \Leftrightarrow V(f) \geqslant V(g)$. If such a representing function exists then $\succcurlyeq$ is a weak order, i.e. $\succcurlyeq$ is complete ( $f \succcurlyeq g$ or $g \succcurlyeq f$ for all acts $f, g$ ) and transitive.

One of the best known representations is subjective expected utility (SEU). It holds whenever the representing function has the following form:

$$
\left(f_{1}, \ldots, f_{n}\right) \mapsto \sum_{i=1}^{n} p_{i} \cdot U\left(f_{i}\right) .
$$

The subjective probabilities $p_{i}$, for $i=1, \ldots, n$ are uniquely determined; they are nonnegative and their sum equals 1 . The utility function $U$ maps from the set of outcomes into the 
reals. In this chapter we consider only continuous and strictly increasing utility functions. In the above formula $U$ is cardinal, i.e. unique up to a positive linear transformation. SEU received much attention for several decades. The first complete axiomatic characterization of SEU was provided by Savage (1954), and many after him formulated preference conditions describing SEU (e.g. Anscombe \& Aumann 1963, Wakker 1984, 1989, d 'Aspremont \& Gevers 1990, Gul 1992).

Choquet expected utility (CEU) holds if the representing function has the following form:

$$
\left(f_{\mathrm{l}}, \ldots, f_{n}\right) \mapsto \sum_{i=1}^{n} \pi_{\rho, i} \cdot U\left(f_{\rho(i)}\right) .
$$

Hore, outcom's are first rank-ordered and then they are valued by the representing function. $U$ is agg:in cardinal. The $\pi_{\rho, j}$, for $j=1, \ldots, n$ are decision weights defined as follows

$$
\pi_{p, i}=\nu(\{\rho(1), \ldots, \rho(i)\})-\nu(\{\rho(1), \ldots, \rho(i-1)\}),
$$

where $\nu: 2^{S} \rightarrow[0,1]$ is a capacity, i.e. $\nu(\emptyset)=0, \nu(S)=1$ and $\nu(A \bigcup B) \geqslant \nu(A)$ for all events $A, B$. Under $C E U$ the capacity is unique. Consequently, the decision weights are uniquely determined, they are nonnegative and sum to one for each permutation $\rho$.

Capacities are nonadditive extensions of probability measures, thus, whenever $\nu$ in (5.1) is additive, CEU reduces to SEU above. CEU was introduced by Schmeidler (1989) for decision under uncertainty (see also Gilboa 1987, Wakker 1989, Nakamura 1990, Chew \& Karni 1994).

In this chapter we focus on cumulative prospect theory (CPT). The representing CPTfunction is defined next. Let $f$ be an act such that for some $k \in\{0, \ldots, n\}$ and $\rho$ we have

$$
f_{\rho(1)} \geqslant \cdots \geqslant f_{\rho(k)} \geqslant 0>f_{\rho(k+1)} \geqslant \cdots \geqslant f_{\rho(n)},
$$

where $k=0$ means that all outcomes are negative and $k=n$ means that all outcomes are nonnegative. The CPT-function has the following form

$$
\begin{aligned}
\left(f_{1}, \ldots, f_{n}\right) \mapsto & \sum_{i=1}^{k} \pi_{\rho, i}^{+} \cdot U\left(f_{\rho(i)}\right) \\
& +\sum_{j=k+1}^{n} \pi_{\rho, j}^{-} \cdot U\left(f_{\rho(j)}\right) .
\end{aligned}
$$

Here the continuous strictly increasing utility function $U$ is required to satisfy $U(0)=0$, and is a ratio scale, i.e. $U$ is unique up to multiplication by a positive real number. For 
the decision weights we have different uniquely determined capacities: $\nu^{+}$for gains and $\nu^{-}$for losses. They are defined as follows. For $i \leqslant k$ (the gain-part of $f$ ) we have similarly to CEU

$$
\pi_{\rho, i}^{+}=\nu^{+}(\{\rho(1), \ldots, \rho(i)\})-\nu^{+}(\{\rho(1), \ldots, \rho(i-1)\}) .
$$

For $j>k$ (the loss-part of $f$ ) we have

$$
\pi_{\rho, j}^{-}=\nu^{-}(\{\rho(j), \ldots, \rho(n)\})-\nu^{-}(\{\rho(j+1), \ldots, \rho(n)\}) .
$$

The CPT-value in (5.2) can be viewed as the sum of two CEU-values. The first sum is the CEU-value of the gain-part $f^{+}$with respect to the capacity $\nu^{+}$, and the second sum is the CEU-value of the loss-part $f^{-}$with respert to the dual of $\nu^{-}$(recall that here $U(0)=0$ ). The CEU-form for losses coincides with the original CEU-form, when in the definition of the decision weights $\nu^{-}(\{\rho(j), \ldots, \rho(n)\})$ is replaced by $1-\nu^{-}(S \backslash\{\rho(1), \ldots, \rho(j-1)\})$.

Moreover, we can write

$$
C P T(f)=C P T\left(f^{+}\right)+C P T\left(f^{-}\right)
$$

in agreement with Tversky \& Kahneman (1992). Aggregating these two values results in the final "worth" of the act. This leature is also exhibited in the proofs (see Appendix). First CPT will be established for gains and then CPT for losses is derived. Then, both parts merge into the general CPT-function.

In this section first preference axioms are formulated such that the utility function in (5.2) becomes an increasing "linear/exponential" function. A function $U: \mathbb{R} \rightarrow \mathbb{R}$ is from the increasing linear/exponential family for gains (losses) if one of the following holds for all $x \geqslant 0(x \leqslant 0)$ :

(i) $U(x)=\alpha \cdot x$, with $\alpha>0$,

(ii) $U(x)=\alpha \cdot e^{\lambda x}+\tau$, with $\alpha \cdot \lambda>0$ and $\tau \in \mathbb{R}$.

Under CPT utility satisfies $U(0)=0$. Therefore, in (i) we dropped the location parameter, and in (ii) the only possibility for the location parameter is $\tau=-\alpha$. In the above definition only the form of utility is described. Clearly the parameters $\alpha, \beta, \lambda$ can be different for gains than for losses.

The central property for a preference relation $\succcurlyeq$ to identify utility as a linear/exponential function is constant absolute risk aversion for gains and for losses. For gains (losses) it is defined as follows

$$
\left(f_{1}, \ldots, f_{n}\right) \succcurlyeq\left(g_{1}, \ldots, g_{n}\right) \Rightarrow\left(f_{1}+\varepsilon, \ldots, f_{n}+\varepsilon\right) \succcurlyeq\left(g_{1}+\varepsilon, \ldots, \bar{g}_{n}+\varepsilon\right),
$$


whenever for all $i=1, \ldots, n$ the outcomes $f_{i}, f_{i}+\varepsilon, g_{i}, g_{i}+\varepsilon$ are gains (losses).

Before formulating the next preference condition some notation is introduced. For an event $I \subseteq S$ and $f, h \in \mathbb{R}^{n}$ by $h_{I} f$ we denote the act which results from $f$ by replacing $f_{i}$ with $h_{i}$ for each state $i \in I$; for states we write $h_{j} f$ instead of $h_{\{j\}} f$. We can now introduce the independence property for $\succcurlyeq$.

DEFINITION The preference relation $\succcurlyeq$ on $\mathbb{R}^{n}$ satisfies tail independence if the following holds:

$$
a_{I} f \succcurlyeq a_{I} g \Leftrightarrow b_{I} f \succcurlyeq b_{I} g
$$

whenever $I=\{\rho(1), \ldots, \rho(m)\}$ or $I=\{\rho(l), \ldots, \rho(n)\}$ for some $m, l \in S$, and all acts in question are from the same set $I R_{\rho}^{n}$.

Tail independence requires that the preference between two acts is independent of common outcomes if, first, the acts are rank-ordered with respect to the same permutation. and second, if after rank-ordering those acts have their common outcomes placed in the first $m$ or last $(n-i+1)$ consecutive states. Thus, tail independence not only restricts the sure thing principle of Savage (1954) to comonotonic acts (called comonotonic independence in Chew \& Wakker, 1996), but in addition it further restricts comonotonic independence to hold for states in which common outcomes are best or worst. Cumulative prospect theory satisfies tail independence as is shown in the next lemma. In order to clarify the nature of CPT the proof is added into the main text.

\section{LEMMA 5.1 CPT implies tail independence.}

Proof: Under CPT we have

$$
f \geqslant g \Leftrightarrow C P T\left(f^{+}\right)+C P T\left(f^{-}\right) \geqslant C P T\left(g^{+}\right)+C P T \cdot\left(g^{-}\right),
$$

for all acts $f, g \in \mathbb{R} R^{n}$.

Let $f, g \in \mathbb{R}_{\rho}^{n}$ for some permutation $\rho$ of the states. The following is implied by the above equivalence

$$
\sum_{i=1}^{k} \pi_{\rho, i}^{+} \cdot U\left(f_{\rho(i)}\right)+\sum_{i=k+1}^{n} \pi_{\rho, i}^{-} \cdot U\left(f_{\rho(i)}\right) \geqslant \sum_{i=1}^{k} \pi_{\rho, i}^{+} \cdot U\left(g_{\rho(i)}\right)+\sum_{i=k+1}^{n} \pi_{\rho, i}^{-} \cdot U\left(g_{\rho(i)}\right) .
$$

Assuming now that $f, g$ have common best outcomes, i.e. for some $m \in S$ we have $f_{\rho(i)}=g_{\rho(i)}$ for all $i=1, \ldots, m$, we conclude that the inequality is independent of the 
first $m$ summinds. Hence, for $i \in I:=\{1, \ldots, m\}$, we can replace $f_{\rho(i)}, g_{\rho(i)}$ by arbitrary common outcomes $h_{\rho(i)}$ without affecting this inequality. Therefore, we can choose $h_{\rho(i)}$, such that $h_{\rho\langle I\rangle} f, h_{\mu(I), g} \in \mathbb{R}_{\rho}^{n}$, implying

$$
f \succcurlyeq g \Leftrightarrow h_{\rho(I)} f \geqslant h_{\rho(I)} g .
$$

Because $\rho$ and $m$ are arbitrarily chosen, independence of common best outcomes holds. Similarly we can show that independence holds for common worst outcomes, which then implies tail independence of $\succcurlyeq$, and thus completes the proof.

The preference relation $\succcurlyeq$ on $\mathbb{R}^{n}$ satisfies monotonicity if $f \succ g$ whenever $f_{i} \geqslant g_{i}$ for all states $i$ with a strict inequality for at least one state.

The continuity condition defined here is with respect to the Euclidean topology on $\mathbb{R}^{n}$ : $\succcurlyeq$ satisfies continuity if for any act $f$ the sets $\left\{g \in \mathbb{R}^{n} \mid g \succcurlyeq f\right\}$ and $\left\{g \in \mathbb{R}^{n} \mid g \preccurlyeq f\right\}$ are closed subsets of $\mathbb{R}^{n}$.

THEOREM 5.2 Assume $n \geqslant 3$. For a preference relation $\geqslant$ on $\mathbb{R}^{n}$ the following two statements are equivalent:

(i) CPT holds, with a continuous strictly increasing linear/exponential utility and positive decision weights.

(ii) The preference relation satisfies the following conditions: weak ordering, monotonicity, continuity, tail independence, and constant absolute risk aversion for gains and for losses.

If (i) holds then the capacities are uniquely determined and the utility function is a ratio scale.

Next, we concentrate on the CPT-model with "power" utility. It is the most-used nonexpected utility form nowadays. For references see Wakker and Zank (1998b), (or Chapter 4). They provided an axiomatization of CPT with power utility for decision under risk. Here an extension of their results to decision under uncertainty is given.

A function $U: \mathbb{R} \rightarrow \mathbb{R}$ is from the positive power family for gains if

$$
U(x)=\alpha^{+} \cdot x^{\lambda^{+}}, \text {with } \alpha^{+}, \lambda^{+}>0, \text { for all } x \geqslant 0,
$$


and it is from the positive power family for losses if

$$
U(x)=-\alpha^{-} \cdot|x|^{\lambda^{-}}, \text {with } \alpha^{-}, \lambda^{-}>0, \text { for all } x \leqslant 0 .
$$

Recall that under CPT we require strict increasingness and $U(0)=0$ for the utility function. Hence, in the above definitions all parameters are positive and no location parameter is added.

The property of $\succcurlyeq$ which determines power utility is constant proportional risk aversion for gains and for losses. For gains (losses) it is defined as follows

$$
\left(f_{1}, \ldots, f_{n}\right) \succcurlyeq\left(g_{1}, \ldots, g_{n}\right) \Rightarrow\left(\varepsilon \cdot f_{1}, \ldots, \varepsilon \cdot f_{n}\right) \succcurlyeq\left(\varepsilon \cdot g_{1}, \ldots, \varepsilon \cdot g_{n}\right),
$$

for all $\varepsilon>0$ whenever all outcomes are gains (losses).

THEOREM 5.3 Assume $n \geqslant 3$. For a preference relation $\succcurlyeq$ on $I R^{n}$ the following two statements are equivalent:

(i) CPT holds, with a positive power utility for gains and for losses, and positive decision weights.

(ii) The preference relation satisfies the following conditions: weak ordering, monotonicity, continuity, tail independence, and constant proportional risk aversion for gains and for losses.

If (i) holds then the capacities are uniquely determined, and the utility function is a ratio scale.

\subsection{Cumulative Prospect Theory with Multilinear and with Additive/Multiplicative Utility}

Recall that in this chapter we consider a finite set of states, $S=\{1, \ldots, n\}$ for a natural number $n \geqslant 3$, where exactly one state is true and there is uncertainty about which is the true state. An act $f$ assigns to each state $j$ an outcome $f_{j}$. In this section we denote the set of outcomes by $X$, and $X$ is the product of a finite number of nondegenerate intervals $X_{1}, \ldots, X_{r}, r \geqslant 2$, called attribute sets. An outcome $x \in X$ can be written as a tuple 
$x=\left(x^{1}, \ldots, x^{r}\right)$ with attribute $x^{t} \in X_{t}$. Again we can view the set of acts as the product $X^{n}$. Sometimes we identify the constant act $(x, \ldots, x) \in X^{n}$ with the outcome $x \in X$.

For simplicity, we assume that each attribute $X_{t}$ contains the zero value in its interior. Therefore, $X$ contains the zero outcome, which is the status quo. Actually any other outcome in $X$ can play the role of the status quo. However, by rescaling the values in each attribute set we can ensure that the zero outcome becomes the status quo. Moreover, for money, zero as status quo is widely accepted in empirical work (see Kahneman \& Tversky, 1979).

On the set of acts $X^{n}$ we assume a preference relation denoted by $\succcurlyeq$. The restriction of $\succcurlyeq$ to the constant acts (and therefore to $X$ ) is also denoted by $\succcurlyeq$. Gains and losses are now defined not only with respect to the status quo, but also depend on the preference relation $\succcurlyeq$ on $X$. Outcomes $x \succ 0$ are gains and outcomes $x \prec 0$ are losses. Note that here gains and losses can contain both positive and negative attributes. Therefore, the "aggregated worth" of an outcome among its $r$ attributes indicates if the outcome is a gain or a loss or is indifferent to the status quo.

Rank-ordering is also defined with respect to the preference relation $\succcurlyeq$ on $X$ : an act $f=\left(f_{1}, \ldots, f_{n}\right)$ is rank-ordered if $f_{1} \succcurlyeq \cdots \succcurlyeq f_{n}$. Similar to Section 5.2 we denote by $X_{p}^{n}$ the set of acts that are rank-ordered according to $\rho$, where $\rho$ is a permutation of the states $\{1, \ldots, n\}$.

Weak ordering: continuity and tail-independence are defined analogously to Section 5.2. The preference relation $\succcurlyeq$ on $X^{n}$ satisfies outcome-monotonicity if for all acts $f, g \in X^{n}$, $f_{i} \succcurlyeq g_{i}$ for all states $i$ implies $f \succcurlyeq g$, with a strict preference if for a state $j$ we have $f_{j} \succ g_{j}$. The preference relation $\geqslant$ on $X^{n}$ satisfies attribute-monotonicity if for all outcomes $x, y \in X,\left[x \neq y\right.$ and $x^{t} \geqslant y^{t}$ for all $\left.t=1, \ldots, r\right]$ implies $x \succ y$.

For a subset $T$ of $\{1, \ldots, r\}$ and outcomes $z, x \in X$ we define $z^{T} x$ as the outcome with attribute $z^{t}$ for $t \in T$ and $x^{t}$ for $t \in T^{c}$, where $T^{c}:=\{1, \ldots, r\} \backslash T$. We denote by $X_{T}$ a factor, which is defined as $X_{T}:=\left\{X_{t} \mid t \in T\right\}$. Instead of $X_{\{t\}}$ we use $X_{l}$. In this section the central property is utility independence for factors restricted to rank-ordered sets, defined next:

Definition Let $T \subseteq\{1, \ldots, r\}$. The factor $X_{T}$ is utility independent for gains (losses) if

$$
\begin{aligned}
\left(x^{T^{c}} f_{1}, \ldots, x^{T^{c}} f_{n}\right) & \succcurlyeq\left(x^{T^{c}} g_{1}, \ldots, x^{T^{c}} g_{n}\right) \\
& \Leftrightarrow
\end{aligned}
$$




$$
\left(y^{T^{e}} f_{1}, \ldots, y^{T^{c}} f_{n}\right) \succcurlyeq\left(y^{T^{c}} g_{1}, \ldots, y^{T^{e}} g_{n}\right)
$$

holds, whenever all acts in question are contained in $X_{\rho}^{n}$ for some $\rho$, and all outcomes are gains (losses).

This property determines the following family of utility functions (Theorem 5.4). A function $U: X \rightarrow \mathbb{R}$ is multilinear if there exist functions $U_{t}: X_{t} \rightarrow \mathbb{R}$ for $t=1, \ldots, r$ and constants $\delta_{T} \in \mathbb{R}$ for all $T \subseteq\{1, \ldots, r\}$ such that $U\left(x^{1}, \ldots, x^{r}\right)=\sum_{T \subseteq\{1, \ldots, r\}} \delta_{T}$. $\prod_{t \in T} U_{t}\left(x^{t}\right)$.

Utility independence is a central tool in Keeney \& Raiffa's (1976) multiattribute utility theory. We define the property not only according to the separation into gains and losses but also restrict it to rank-ordered acts.

ThEOREM 5.4 Assume $n \geqslant 3$. For a preference relation $\succcurlyeq$ on $X^{n}$ the following two statements are equivalent:

(i) CPT holds, with a continuous multilinear utility, strictly increasing in each attribute, and with positive decision weights.

(ii) The preference relation satisfies the following conditions: weak ordering, outcomemonotonicity, attribute-monotonicity, continuity, tail independence, and for each factor $X_{\iota}, t=1, \ldots, r$, utility independence for gains and for losses.

If (i) holds then the capacities are uniquely determined and the utility function is a ratio scale.

Mutual utility independence holds for gains (losses) whenever $X_{T}$ is utility independent for gains (losses) for all $T \subseteq\{1, \ldots, r\}$. This property characterizes additive and/or multiplicative utilities (Theorem 5.5). A function $U: X \rightarrow \mathbb{R}$ is additive if $U\left(x^{1}, \ldots, x^{r}\right)=$ $\sum_{t=1}^{r} U_{t}\left(x^{t}\right)$ and is multiplicative if $U\left(x^{1}, \ldots, x^{r}\right)=\prod_{t=1}^{r} U_{t}\left(x^{t}\right)$, where $U_{t}: X_{t} \rightarrow I R$ for all $t=1, \ldots, r$. A function is additive/multiplicative if it is either additive or multiplicative.

THEOREM 5.5 Assume $n \geqslant 3$. For a preference relation $\succcurlyeq$ on $X^{n}$ the following two statements are equivalent:

(i) CPT holds, with a continuous additive/multiplicative utility, strictly increasing in each attribute, and with positive decision weights. 
(ii) The preference relation satisfies the following conditions: weak ordering, outcomemonotonicity, attribute-monotonicity, continuity, tail independence, and mutual utility independence for gains and for losses.

If (i) holds then the capacities are uniquely determined and the utility function is a ratio scale.

The above theorem identifies the conditions that are needed for the existence of an additive or multiplicative utility function $U$. Conditions that distinguishes the additive and the multiplicative representation were formulated by Miyamoto (1988), Miyamoto \& Wakker (1996) for acts from a two attribute set, and was extended in Bleichrodt \& Quiggin (1997) for acts from finite attribute sets. All these conditions are strengthenings of utility independence for factors and are satisfied if and only if the additive utility form applies. This is shown in Miyamoto (1988), Miyanuto \& Wakker (1996) and Bleichrodt \& Quiggin (1997) for two-state spaces. Such a condition can be adapted here for any finite number states for the distinction of additive and multiplicative utility representations. However, this point is not elaborated here.

\subsection{Appendix: Proofs to Chapter 5}

Proof of Theorem 5.2. First, Statement (i) is assumed, and Statement (ii) is concluded: CPT holds for $\succcurlyeq$ on $\mathbb{I R}^{n}$ with continuous increasing linear/exponential utility. Weak ordering is immediate from the existence of the representing CPT-function for $\succcurlyeq$. Monotonicity holds because utility is increasing and the decision weights are positive. Continuity of utility implies continuity of $\succcurlyeq$. Tail independence holds by Lemma 5.1. Finally, constant absolute risk aversion for gains and losses is implied by the nature of the utility function. This completes the proof of Statement (ii).

Next, Statement (ii) is assumed, and Statement (i) is derived. The proof consists of several intermediate results. First, it is shown that on the set of rank-ordered acts $\mathbb{I R}_{\text {id }}^{n}$ the preference relation is represented by the additive function described in Lemma 5.6. Then (Lemma 5.7), it is shown that the additive function in Lemma 5.6, when restricted to rankordered acts with nonnegative outcomes, agrees with the restriction of a CEU-function, where utility is linear/exponential and the decision weights are positive. Similarly, in Lemma 5.8 it is shown that the additive function of Lemma 5.6, when restricted to rankordered acts with nonpositive outcomes, agrees with a $\mathrm{CEU}$-restriction, where utility is 
linear/exponential and the decision weights are positive. In Lemma 5.7 similar results are derived for $\succcurlyeq$ on $\mathbb{R}_{p}^{n}$, for each pernautation $\rho$ of the states. Then, it is shown that the different restrictions fit together into a general function, such that on $\mathbb{R}_{+}^{n}$ (the set of acts with nonnegative outcomes) and on $I R_{-}^{n}$ (the set of acts with nonpositive outcomes) Choquet expected utility holds, and thus on $I R^{n}$ CPT holds for $\succcurlyeq$. We complete the proof of Statement (i) by deriving uniqueness results.

LEMMA 5.6 The preference relation $\succcurlyeq$ on $\mathbb{I R}_{i d}^{n}$ is represented by the additive function

$$
\left(f_{1}, \ldots, f_{n}\right) \mapsto \sum_{j=1}^{n} V_{j}\left(f_{j}\right),
$$

with continuous strictly increasing functions $V_{1}, \ldots, V_{n}: \mathbb{R} \rightarrow \mathbb{R}$, which are uniquely determined satisfying $V_{j}(0)=0$ for all $j$ and $\sum_{j=1}^{n} V_{j}(1)=1$.

Proof. The proof follows immediately from Lemma 7 in Wakker \& Zank (1998b), (see also Lemma 4.6). There, the statement is formulated for a preference relation on a set of simple lotteries (i.e. finite probability distributions over $I R$ ) with rank-ordered outcomes. However, they fix a finite probability distribution, such that only outcomes can vary, which results in a set isomorphic to $I R_{i d}^{n}$. Then our statement results.

LEMMA 5.7 On the set of rank-ordered acts with nonnegative outcomes the representation of Lemma 5.6 agrees with the following function

$$
C E U_{i d}^{+}:\left(f_{1}, \ldots, f_{n}\right) \mapsto \sum_{j=1}^{n} \pi_{i d, j}^{+} \cdot U_{i d}\left(f_{j}\right)
$$

where $U_{\text {id }}$ is a strictly increasing linear/exponential utility function, satisfying $U_{\text {id }}(0)=0$ and $U_{i d}(1)=1$, and the decision weights $\pi_{i d, j}^{+}$, for $j=1, \ldots, n$, are all positive. Utility and the decision weights are uniquely determined.

ProOF. We have given the preference relation $\succcurlyeq$ on $I R_{i d}^{n}$, which is represented by the function $\sum_{j=1}^{n} V_{j}$ with the $V_{j}$ 's as described in Lemma 5.6. Moreover $\%$ satisfies constant absolute risk aversion on $\backslash R_{++, i d}^{n}$, i.e. the set of rank-ordered acts with positive outcomes.

We define $U_{i d}(x)=\sum_{j=1}^{n} V_{j}(x)$ for all nonnegative $x$. Therefore, by Lemma $5.6 U_{i d}$ becomes unique satisfying $U_{\text {id }}(0)=0, U_{\text {id }}(1)=1$.

Let us fix some $1 \leqslant i<n$. We restrict our analysis to acts with identical outcomes for the first $i$ states and for the last $n-i$ states, i.e. to acts $f$ with $f_{j}=x$ for $j=1, \ldots, i$ 
and $f_{j}=y$ for $j=i+1, \ldots, n$, for outcomes $x, y$ with $x \geqslant y \geqslant 0$. We denote these acts by $(x, y)_{i}$. On this two-dimensional subset the preference relation is represented by $(x, y)_{i} \mapsto Z_{i}(x)+W_{i}(y)$, with $Z_{i}:=\sum_{j=1}^{i} V_{j}$ and $W_{i}:=\sum_{j=i+1}^{n} V_{j}$. Constant absolute risk aversion for gains implies that $\succcurlyeq$ on this subset is invariant w.r.t. addition of a common constant to all outcomes (whenever the resulting acts remain in this subset). Considering only gains $(y>0)$, Miyamoto \& Wakker (1996, Theorem 1) show that $Z_{i}$ and $W_{i}$ are proportional, which obviously remains valid when the zero outcome is included. Moreover, $Z_{i}$ and $W_{i}$ are proportional to their sum, which is $U_{i d}$. Therefore, by Miyamoto \& Wakker, they are of the form $Z_{i}=\pi_{Z_{i}} \cdot U_{i d}$ and $W_{i}=\pi_{W_{i}} \cdot U_{i d}$, for positive uniquely determined $\pi_{Z_{i}}, \pi_{W_{i}}$, which sum to one. Further, Miyamoto \& Wakker concluded that the utility function $U_{i d}$ is from the increasing linear/exponential family for gains. This analysis holds for any fixed $1 \leqslant i<n$.

We define now $\pi_{i d, 1}^{+}:=\pi_{Z_{1}}, \pi_{i d, j}^{+}:=\pi_{Z_{j}}-\pi_{Z_{j-1}}$ for $j=2, \ldots, n-1$, and $\pi_{i d, n}^{+}:=\pi_{W_{n-1}}$. Monotonicity implies that all the $\pi_{i, j, j}^{+}$'s are positive. By their definition they are uniquely determined and sum to one. Then, we can compute $V_{1}=Z_{1}=\pi_{i d, 1}^{+} \cdot U_{s d}$, and inductively $V_{j}=Z_{j}-Z_{j-1}=\pi_{i d, j}^{+} \cdot U_{i d}$ for $j=2, \ldots, n-1$, and $V_{n}=W_{n-1}=\pi_{i d, n}^{+} \cdot U_{i d l}$.

Finally, from this analysis, we conclude that on $\mathbb{R}_{+, i d}^{n}$ the representation of Lemma 5.6 agrees with the function in (5.3). This concludes the proof of Lemma 5.7.

LEMMA 5.8 On the set of rank-ordered acts with nonpositive outcomes the representation of Lemma 5.6 agres with the following function

$$
C E U_{i d}^{-}:\left(f_{1}, \ldots, f_{n}\right) \mapsto \sum_{j=1}^{n} \pi_{i d, j}^{-} \cdot U_{i d}\left(f_{j}\right),
$$

where $U_{i d}$ is a strictly increasing linear/exponential utility function, satisfying $U_{i d}(0)=0$, and the decision weights $\pi_{i d, j}^{-}$, for $j=1, \ldots, n$, are all positive. Utility and the decision weights are uniquely determined.

Proof. The proof is similar to the one of Lemma 5.7. We can view the "problem" $\left\{S, I R_{-, i d}^{n} \succcurlyeq, \sum_{j=1}^{n} V_{j}(\cdot)\right\}$ as an equivalent problem $\left\{S^{*}, I R_{+, i d}^{n}, \succcurlyeq^{*},-\sum_{j=n}^{1} V_{j}(-(\cdot))\right\}$. Now $S^{*}:=\{n, \ldots, 1\}$, an act $\left(f_{1}, \ldots, f_{n}\right) \in \mathbb{I R}_{-, i d}^{n}$ corresponds to an act $\left(-f_{n}, \ldots,-f_{1}\right) \in I R_{+, i d}^{n}$, and the preference relation $\succcurlyeq *$ is defined as follows:

$$
\left(-f_{n}, \ldots,-f_{1}\right) \succcurlyeq *\left(-g_{n}, \ldots,-g_{1}\right) \Leftrightarrow\left(f_{1}, \ldots, f_{n}\right) \preccurlyeq\left(g_{1}, \ldots, g_{n}\right) .
$$


Therefore $\succcurlyeq *$ is a continuous monotonic weak order satisfying tail independence and constant absolute risk aversion for gains. Moreover, $\succcurlyeq^{*}$ is represented by

$$
\left(-f_{n}, \ldots,-f_{1}\right) \mapsto-\sum_{j=n}^{1} V_{j}\left(f_{j}\right),
$$

with the $V_{j}$ 's as described in Lemma 5.6. It can now be demonstrated, following the lines in the proof of Lemma 5.7, that similar results as in Lemma 5.7 hold here. Reformulation in terms of the problem $\left\{S, \mathbb{I}_{-, i d}^{n}, \succcurlyeq, \sum_{j=1}^{n} V_{j}(\cdot)\right\}$ gives exactly the statement of Lemma 5.8. This completes the proof.

LEMMA 5.9 For each permutation $\rho$ of $\{1, \ldots, n\}$ the preference relation $\geqslant$ on $\mathbb{R}_{\rho}^{n}$ is represented by an additive function

$$
\left(f_{1}, \ldots, f_{n}\right) \mapsto \sum_{j=1}^{n} V_{j}^{\rho}\left(f_{\rho(j)}\right),
$$

with continuous strictly increasing functions $V_{1}^{\rho}, \ldots, V_{n}^{\rho}: \mathbb{R} \rightarrow \mathbb{R}$, which are uniquely determined satisfying $V_{j}^{\rho}(0)=0$ for all $j$ and $\sum_{j=1}^{n} V_{j}^{\rho}(1)=1$. The additive function described above agrees on $\mathbb{I R}_{+, \rho}^{n}$ with the following function

$$
C E U_{\rho}^{+}:\left(f_{1}, \ldots, f_{n}\right) \mapsto \sum_{j=1}^{n} \pi_{\rho, j}^{+} \cdot U_{p}\left(f_{\rho(j)}\right),
$$

and on $I R_{-, \rho}^{n}$ with the function

$$
C E U_{\rho}^{-}:\left(f_{1}, \ldots, f_{n}\right) \mapsto \sum_{j=1}^{n} \pi_{\rho, j}^{-} \cdot U_{\rho}\left(f_{\rho(j)}\right)
$$

The utility function $U_{\rho}$ is from the increasing linear/exponential family for both gains and losses, satisfying $U_{\rho}(0)=0$ and $U_{\rho}(1)=1$, and the decision weights $\pi_{\rho, j}^{+}, \pi_{\rho, j}^{-}$, for $j=1, \ldots, n$, are all positive. Utility and the decision weights are uniquely determined.

ProOF. Take any permutation $\rho$ of $\{1, \ldots, n\}$. The preference relation $\succcurlyeq$ on $\mathbb{R}_{p}^{n}$ is a continuous monotonic weak order, satisfying tail independence and constant absolute risk aversion for gains and for losses. The proofs of Lemma 5.6, 5.7 and 5.8 can be adapted, considering instead of $S$ the "reordered" set of states $S_{\rho}:=\left\{\rho^{-1}(1), \ldots, \rho^{-1}(n)\right\}$.

We have now obtained representations for $\succcurlyeq$ on each set $\mathbb{R}_{\rho}^{n}$, which, on $I R_{+, \rho}^{n}$ and $I R_{-, \rho}^{n}$ agree with restrictions of CEU-functions as described in Lemma 5.9. The next step in the 
proof of Theorem 5.2 is to show that the different functions have identical utility. We show this only for gains. A similar result can be proved for losses.

Let $\rho$ be a permutation of the states $\{1, \ldots, n\}$, such that the set $I R_{+, \rho}^{n} \cap I R_{+, i d}^{n}$ contains nonconstant acts. Then, $C E U_{p}^{+}$and $C E U_{i d}^{+}$jointly represent the preference relation $\succcurlyeq$ on the intersection $\mathbb{R}_{+, \rho}^{n} \cap \mathbb{R}_{+, i d}^{n}$. However, any additive representation of $\succcurlyeq$ on $\mathbb{R}_{+, \rho}^{n} \cap$ $\mathbb{R}_{+, i d}^{n}$, because cardinal (see for instance Chateauneuf \& Wakker, 1993), becomes unique by fixing scale and location. Moreover, by reasonings similar to Lemma 5.9 , uniquely determined utility and decision weights can be derived for such a representation. Thus, the representation for $\succcurlyeq$ on $\mathbb{I R}_{+, \rho}^{n} \cap \mathbb{I R}_{+, i d}^{n}$, with unique utility and decision weights, is a

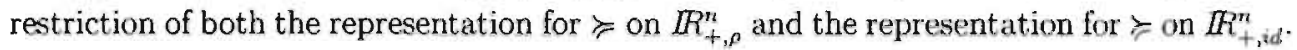
Consequently, the utilities for botb extensions are identical and we conclude that $U_{q}=U_{i d}$ on $\mathbb{R}$, whenever $\mathbb{R}_{+, \rho}^{n} \cap \mathbb{R}_{+, i d}^{n}$ contains nonconstant acts.

Now let $\rho$ be a permutation of $\{1, \ldots, n\}$, such that the set $\mathbb{R}_{+, p}^{n} \cap \mathbb{R}_{+, i d}^{n}$ contains only constant acts. Then, using the fact that $n \geqslant 3$, one can easily construct a sequence of permutations $\rho_{1}, \rho_{2}, \rho_{3}$ of the states such that all sets $\mathbb{I} R_{+, \rho}^{n} \cap \mathbb{I} R_{+, \rho_{1}}^{n}, \mathbb{R}_{+, \rho_{1}}^{n} \cap \mathbb{R}_{+, \rho_{2}}^{n}$, $\mathbb{R}_{+, \rho_{2}}^{n} \cap \mathbb{R}_{+, \rho_{3}}^{n}, \mathbb{R}_{+, \rho_{3}}^{n} \cap \mathbb{R}_{+, i d}^{n}$ contain nonconstant acts. Hence, by the analysis before, we conclude that the utilities for $C E U_{\rho}^{+}$and $C E U_{i d}^{+}$are identical.

Therefore, we conclude that the different functions derived in Lemma 5.9, have the same utility function $U$, and hence assign the same value to constant acts, i.e. the acts which are commonly contained in all sets $I R_{p}^{n}$. Using this result, the following holds:

Lemma 5.10 For each act $f \in I R_{+}^{n}$ or $I R_{-}^{n}$ there exists a certainty equivalent, i.e. a constant act $x_{f}$ with $f \sim x_{f}$.

Proof. (only for the case $f \in \mathbb{R}_{+}^{n}$ ) Take any act $f \in \mathbb{R}_{+, \rho}^{n}$. Let $x$ be the maximal and $y$ the minimal outcome of $f$. Monotonicity of $\succcurlyeq$ implies $x \succcurlyeq f \succcurlyeq y$. Thus, the following equivalence holds

$$
C E U_{p}^{+}(x) \geqslant C E U_{\rho}^{+}(f) \geqslant C E U_{\rho}^{+}(y) \Leftrightarrow U(x) \geqslant C E U_{\rho}^{+}(f) \geqslant U(y),
$$

and, by continuity of $U$, there exists $x_{f} \in \mathbb{R}_{+}$, with $C E U_{\rho}^{+}(f)=U\left(x_{f}\right)$. By monotonicity, $x_{f}$ is unique. Hence, the constant act $x_{f}$ is indifferent to $f$.

Now we show the existence of a representing CEU-function for $\succcurlyeq$ on $\mathbb{R}_{+}^{n}$. Take any acts $f, g \in \mathbb{R}_{+}^{n}$. Let be $f \in \mathbb{R}_{+, p}^{n}, g \in \mathbb{R}_{+, p^{\prime}}^{n}$ and $C E U_{p}^{+}, C E U_{p^{+}}^{+}$the representations for $\succcurlyeq$ on $I R_{+, \rho}^{n}, \mathbb{R}_{+, p^{\prime}}^{n}$, respectively, derived in Lemma 5.9. Then, by Lemma 5.10 we have 
$f \succcurlyeq g \Leftrightarrow f \succcurlyeq x_{g} \Leftrightarrow C E U_{\rho}^{+}(f) \geqslant C E U_{\rho}^{+}\left(x_{g}\right)$. Further, because utility is the same for all CEU-forms, it follows that $C E U_{\rho}^{+}\left(x_{g}\right)=U\left(x_{g}\right)=C E U_{\rho^{\prime}}^{+}\left(x_{g}\right)=C E U_{\rho^{\prime}}^{+}(g)$. Thus, we conclude

$$
f \succcurlyeq g \Leftrightarrow C E U_{\rho}^{+}(f) \geqslant C E U_{\rho^{\prime}}^{+}(g) .
$$

By setting $f=g$, this implies that $C E U_{\rho}^{+}$agrees with $C E U_{\rho^{\prime}}^{+}$on common domain, i.e. on $\mathbb{R}_{+, \rho}^{n} \cap \mathbb{R}_{+, \rho^{\prime}}^{n}$. Thus, they can be considered restrictions of one function. Because $f$ and $g$ were arbitrary, we conclude the existence of a general function, denoted $C E U^{+}$, which represents $\succcurlyeq$ on $I R_{+}^{n}$, and agrees with $C E U_{\rho}^{+}$on $\mathbb{R}_{+, \rho}^{n}$ for any permutation $\rho$ of the states.

Recall that the act $1_{I} 0$ assigns outcome 1 to the states in $I$ and outcome 0 elsewhere. Now define $\nu^{+}(I):=C E U^{+}(1,0)$ for all $I \subseteq\{1, \ldots, n\}$. Obviously, $\nu^{+}$is a capacity on $S$. Moreover the following holds

$$
\pi_{\rho, j}^{+}=\nu^{+}(\rho(1), \ldots, \rho(j))-\nu^{+}(\rho(1), \ldots, \rho(j-1)),
$$

for $j=1, \ldots, n$ and any permutations $\rho$ of the states. Because the decision weights are uniquely determined, by the above definition the capacity $\nu^{+}$is unique.

Let us summarize: The preference relation $\succcurlyeq$ on $\mathbb{R}_{+}^{n}$ is represented by the function $C E U^{+}$which is a Choquet expected utility function as described in (5.1). Further the utility function $U$ is uniquely determined from the increasing linear/exponential family for gains, satisfying $U(0)=0, U(1)=1$, and the capacity $\nu^{+}$on $S$ generates positive decision weights.

Similarly, for the preference relation $\succcurlyeq$ on $\mathbb{R}_{-}^{n}$, we can derive representability by a Choquet expected utility function, now denoted $\mathrm{CEU}^{-}$. The utility function $U$ is from the increasing linear/exponential for losses, it is again unique and satisfies $U(0)=0$. The capacity $\nu^{-}$, now defined by $\nu^{-}(I):=C E U^{-}(-1,0) / U(-1)$ for all $I \subseteq\{1, \ldots, n\}$ is again unique, and it generates positive decision weights. Moreover the following holds for $\nu^{-}$

$$
\pi_{\rho, j}^{-}=\nu^{-}(\rho(j), \ldots, \rho(n))-\nu^{-}(\rho(j+1), \ldots, \rho(n)),
$$

for $j=1, \ldots, n$ and any permutations $\rho$ of the states.

Now let $f$ be an act containing both gains and losses. Suppose $f \in \mathbb{R}_{p}^{n}$ for a permutation $\rho$ of $\{1, \ldots, n\}$. There exists a $k \in\{1, \ldots, n\}$ with

$$
f_{\rho(1)} \geqslant \cdots \geqslant f_{\rho(k)} \geqslant 0>f_{\rho(k+1)} \geqslant \cdots \geqslant f_{\rho(n)} .
$$


Then, with $f^{+}$the gain part of $f, f^{-}$the loss part of $f$, and the $V_{j}^{\rho}$ 's from Lemma 5.9, the following holds

$$
\sum_{j=1}^{n} V_{j}^{\rho}\left(f_{\rho(j)}\right)=\sum_{j=1}^{n} V_{j}^{\rho}\left(f_{\rho(j)}^{+}\right)+\sum_{j=1}^{n} V_{j}^{\rho}\left(f_{\rho(j)}^{-}\right),
$$

which, by the results above, is equivalent to

$$
\sum_{j=1}^{n} V_{j}^{\rho}\left(f_{\rho(j)}\right)=C E U^{+}\left(f^{+}\right)+C E U^{-}\left(f^{-}\right) .
$$

Therefore, the additive representations for $\succcurlyeq$ on $\mathbb{R}_{\rho}^{n}$ described in Lemma 5.9 can be considered as restrictions of a common function, defined by $f \mapsto C E U^{+}\left(f^{+}\right)+C E U^{-}\left(f^{-}\right)$. Obviously, this function represents the preference relation $\succcurlyeq$ on the entire set of acts $I R^{n}$, and it is a CPT-function as described in (5.2), with a increasing linear/exponential utility function for gains and for losses $U$, which satisfies $U(0)=0$ and $U(1)=1$. Utility and the capacities $\nu^{+}, \nu^{-}$are uniquely determined by the analysis made separately for gains and for losses.

Note that in the derivation of the representation above we have always fixed scale and location. Let now $U^{*}, \mu^{+}, \mu^{-}$describe a CPT-function representing $\succcurlyeq$ on $I R^{n}$, with $U^{*}(0)=0$. Thus, only location is fixed for $U^{*}$ but not scale. Then, also $U^{*} / U^{*}(1), \mu^{+}, \mu^{-}$ describes a CPT-function representing $\succcurlyeq$ on $\mathbb{R}^{n}$. Consequently, by the results before, $U^{*} / U^{*}(1)=U$, and $\mu^{+}=\nu^{+}, \mu^{-}=\nu^{-}$follows. Further, if $U, \nu^{+}, \nu^{-}$describe a CPTrepresentation for $\succcurlyeq$ on $\mathbb{R} R^{n}$, then also $\gamma \cdot U, \nu^{+}, \nu^{-}$describe a CPT-representation for $\succcurlyeq$ on $\mathbb{I R}^{n}$ for any positive $\gamma$. This shows that $U$ is a ratio scale, and that the capacities are uniqucly determined. Moreover all generated decision weights are positive. Thus the proof of Statement (ii) is complete.

Hence, we have completed the proof of Theorem 5.2 .

Proof of Theorem 5.3. That Statement (i) implies (ii) is immediate. The proof of (i) from (ii) is analugous to that in the proof of Theorem 5.2. The difference is that for the preference relation here constant absolute risk aversion is replaced by constant proportional risk aversion. In Lemma 5.6 this is not yet relevant, therefore Lemma 5.6 holds here. Constant absolute risk aversion was relevant in Lemma 5.7. Considering constant proportional risk aversion instead, Lemma 5.7 remains valid if we replace " $U_{i d}$ is a strictly increasing linear/exponential utility function" by " $U_{i d}$ ' is from the positive power 
family". Then, in the proof we have to use Theorem 2 of Miyamoto \& Wakker (1996) instead of their Theorem 1. Other than that, the proof can entirely be adapted with a positive power utility $U_{i d}$ instead of a linear exponential one.

Similarly, the Lemmas 5.8 and 5.9 remain valid with power utility instead of linear/exponential utility. Moreover, the analysis following the proof of Lenuma 5.9 can entirely be repeated here, concluding Statement (i) in Theorem 5.3. This completes the proof.

Proof of ThEOREM 5.4. Statement (i) is assumed, and we conclude Statement (ii). Suppose that CPT holds for $\succcurlyeq$ on $X^{n}$, with a continuous multilinear utility, strictly increasing in each attribute, and with positive decision weights. Weak ordering of $\succcurlyeq$ is immediate from the existence of the representing CPT-function for $\succcurlyeq$. Outcome-monotonicity holds because the decision weights are positive. Attribute-monotonicity follows because utility is strictly increasing in each attribute, and because the decision weights are positive. Continuity of utility implies continuity of $\geqslant$. Tail independence is given by Lemma 5.1. Finally, on each set $X_{\rho}^{n_{2}}$ utility independence for each factor $X_{t}, t=1, \ldots, r$, for gains and losses is implied by the nature of the utility function. This completes the proof of Statement (ii).

Now we prove that Statement (ii) implies Statement (i). The proof hereof mainly follows the lines indicated in the proof of Statement (i) from (ii) of Theorem 5.2. I point out differences here, and whenever possible I refer to that proof.

The first difference consists in the existence of "extreme acts". An outcome $x \in X$ is maximal if for no other outcome $y \in X$, we have $y \succ x$, and $x$ is minimal if for no other outcome $y \in X$, we have $y \prec x$. An act assigning to each state a maximal outcome or to each state a minimal outcome is an extreme act. Wakker (1993b) pointed out difficulties for additive representability on rank-ordered sets, in the presence of extreme acts. They had to be excluded in order to derive additive representability. Under proportionality of the functions in the additive representation on the set of nonminimal and nonmaximal outcomes, as will be derived here, extensions to extreme acts were: possible (see Proposition 3.5 in Wakker, 1993b).

LEMMA 5.11 The preference relation $\succcurlyeq$ on $X_{i d}^{n} \backslash\{$ extreme acts $\}$ is represented by the additive function

$$
\left(f_{1}, \ldots, f_{n}\right) \mapsto \sum_{j=1}^{n} V_{j}\left(f_{j}\right)
$$


with continuous functions $V_{1}, \ldots, V_{n}: X \rightarrow \mathbb{R}$, which preserve the ordering of outcomes, and are uniquely determined satisfying $V_{j}(0)=0$ for all $j$ and $\sum_{j=1}^{n} V_{j}(w)=1$ for some fixed $w \succ 0$.

Proof. The proof follows by similar reasonings to those used in the proof of Lemma 7 of Wakker \& Zank (1998b), (see also Lemma 4.6). There it is described how tail independence implies, first locally then globally, the additivity axioms required in Chateauneuf \& Wakker (1993). Then by Corollary C.5 of Chateauneuf \& Wakker additive representability follows.

For $z \in X$ and $t=1, \ldots, r$ we define the sets

$$
X_{t,+}(z):=\left\{x^{t} \in X_{t} \mid x^{t} \geqslant z^{t}\right\} \text { and } X_{t,-}(z):=\left\{x^{l} \in X_{\iota} \mid x^{\ell} \leqslant z^{t}\right\}
$$

Now take $z \in \operatorname{int}(X)$, i.e. the interior of $X$, with $z \sim 0$. We restrict our analysis to rank-ordered acts from $X_{+}^{n}(z) \backslash$ extreme acts $\}$, where $X_{+}(z):=\left[X_{1,+}(z)\right] \times \cdots \times\left[X_{r,+}(z)\right]$. Note that by attribute monotonicity the outcomes in $X_{+}(z)$ are all gains except for $z$, which by its choice is indifferent to the status quo.

LEMMA 5.12 On the set $X_{+, \text {id }}^{n}(z) \backslash\{$ extreme acts $\}$ the representation of Lemma 5.11 agrees with the following function

$$
C E U_{i d, z}^{+}:\left(f_{1}, \ldots, f_{n}\right) \mapsto \sum_{j=1}^{n} \pi_{i d, j, z}^{+} \cdot U_{2 d}^{z}\left(f_{j}\right),
$$

where $U_{\text {ad }}^{z}$ is a multilinear utility function, preserving the ordering on $X$, and increasing in each attribute. $U_{i d i}^{z}$ satisfies $U_{i d}^{z}(z)=0$, and the decision weights $\pi_{i d, j, z}^{+}$for $j=1, \ldots, n$ are all positive. Utility and the decision weights are uniquely determined.

Proof. We have given the preference relation $\succcurlyeq$ on $X_{+, i d}^{n}(z) \backslash\{$ extreme acts $\}$, which is represented by the function $\sum_{j=1}^{n} V_{j}$ with the $V_{j}$ 's as described in Lernma 5.11. Moreover $\succcurlyeq$ satisfies attribute monotonicity and for each attribute set $X_{j_{1+}}(z)$ utility independence for gains on $X_{+, i d}^{n}(z) \backslash\{$ extreme acts $\}$.

We define $U_{i d}^{z}(x)=\sum_{j=1}^{n} V_{j}(x)$ for all $x \in X_{+}(z)$. Therefore, by Lemma 5.11, $U_{i d}^{z}$ becomes unique and satisfies $U_{i d}^{z}(z)=0$.

Now the reasonings are similar to those in the proof of Lemma 5.7. Instead of $x \geqslant y \geqslant 0$ we have $x \succcurlyeq y \succcurlyeq z$, instead of Theorem 1 of Miyamoto \& Wakker (1996) we use Theorem 
5, and monotonicity is replaced by outcome-monotonicity. Except for these changes the proof of Lemma 5.7 can entirely be repeated here.

We conclude that on $X_{+, i d}^{n}(z) \backslash\{$ extreme acts $\}$ the representation of Lemma 5.11 agrees with the function in (5.7). This concludes the proof of Lemma 5.12.

Let now $\hat{z}, \bar{z} \in \operatorname{int}(X)$ with $\bar{z} \sim \tilde{z} \sim 0$ be any distinct outcomes. (Such outcomes exist because $r \geqslant 2$, and because on $X$ the preference relation $\succcurlyeq$ is a continuous weak order, satisfying attribute-monotonicity.) Then, the outcome $y$, defined by $y^{t}:=\max \left\{\hat{z}^{t}, \bar{z}^{t}\right\}$ for all $t=1, \ldots, r$, is contained in $\operatorname{int}(X)$, and by attribute monotonicity satisfies $y \succ 0$.

A similar analysis as in the proof of Lemma 5.12 can be made here, such that we can conclude that on $X_{+, i d}^{n}(y) \backslash\{$ extreme acts $\}$ the additive function in Lemma 5.11 agrees with

$$
C E U_{i d, y}^{+}:\left(f_{1}, \ldots, f_{n}\right) \mapsto \sum_{j=1}^{n} \pi_{i d, j, y}^{+} \cdot U_{i d}^{u}\left(f_{l}\right),
$$

with uniquely determined multilinear utility function $U_{i d}^{y}$ increasing in each attribute, and positive decision weights $\pi_{i d, j, y}^{+}$for $j=1, \ldots, n$.

Now, both $X_{+, i d}^{n}(\hat{z})$ and $X_{+, i d}^{n}(z)$ contain $X_{+, i d}^{n}(y)$, and the latter contains an open subset where $C E U_{i d, z}^{+}$and $C E U_{i d, z}^{+}$both represent the same preference. By the uniqueness of the function in (5.8), we conclude that the utilities and the decision weights in $C E U_{i d, i}^{+}$ and $C E U_{i d, \bar{z}}^{+}$are identical, i.e. $U_{i d}^{\hat{z}}=U_{i d}^{\tilde{z}}$ and $\pi_{i d, j, \hat{z}}^{+}=\pi_{i d, j, z}^{+}$for all $j=1, \ldots, n$. Moreover, because $\hat{z}$ and $\bar{z}$ were arbitrarily chosen from int $(X)$, we conclude that the function in (5.7) is independent of $z$, and thus we can suppress the index $z$ in (5.7).

Recall, that we restricted the above analysis to $z \in \operatorname{int}(X)$ such that $z \sim 0$. Doing so, the outcomes where $U_{i d}$ is not yet defined are boundary outcomes (gains or outcomes indifferent to the status quo) of $X$. But for these boundary outcomes, viewed as constant acts, the function $\sum_{j=1}^{n} V_{j}$ is defined (except for the extreme acts), and thus, we can continuously extend $U_{i d}$ to those outcomes by $U_{i d}:=\sum_{j=1}^{n} V_{j}$, preserving multilinearity.

In Lemma 5.11 we excluded the extreme acts. However, later in the analysis, we concluded that the $V_{j}$ 's in Lemma 5.11 are proportional. Then, by Proposition 3.5 of Wakker (1993b), we can extend $\sum_{j=1}^{n} V_{j}$ to the extreme acts which are gains, and thus, $U_{i d}$ to the entire set $X_{+}$, which is defined to be the union of the set of gains and the set of outcomes indifferent to the status quo. Finally we conclude the following: 
function

$$
C E U_{i d}^{+}:\left(f_{1}, \ldots, f_{n}\right) \leftrightarrow \sum_{j=1}^{n} \pi_{i d, j}^{+} \cdot U_{i d}\left(f_{j}\right),
$$

where $U_{i d}$ is a maltilinear utility function, preserving the ordering on $X_{+}$, and increasing in each altribute; $U_{i d}$ satisfies $U_{i d}(0)=0, U_{u d}(w)=1$; the decision weights $\pi_{i d, j}^{+}$are positive for all $j=1, \ldots$, n. Utility and the decision weights are uniquely determined.

A similar analysis as the one following the proof of Lemma 5.11 can be made for acts from $X_{-, \text {, w }}^{n}$, where $X_{-}$is the union of the set of losses and the set of outcomes indifferent to the status quo, concluding the following:

LEMMA 5.14 On the set $X_{-, \text {id }}^{n}$ the preference relation $\succcurlyeq$ is represented by the following function

$$
C E U_{i d}^{-}:\left(f_{1}, \ldots, f_{n}\right) \mapsto \sum_{j=1}^{n} \pi_{i d, j}^{-} \cdot U_{i d}\left(f_{j}\right),
$$

where $U_{i d}$ is a multilinear utility function, preserving the ordering on $X_{-}$, and increasing in each attribute; $U_{i d}$ satisfies $U_{i d}(0)=0$; the decision weights $\pi_{i d, j}^{-}$are positive for all $j=1, \ldots, n$. Utility and the decision weights are uniquely determined.

Now the rest of the proof of Statement (i) follows the line of the proof of Statement (i) in Theorem 5.2. The reasonings are siruilar, and therefore, we briefly indicate the next steps.

First, for each permutation $\rho$ of the states, on the set $X_{+, \rho}^{n}\left(X_{-, \rho}^{n}\right)$ we can derive representability of $\succcurlyeq$ by a function $C E U_{p}^{+}\left(C E U_{\rho}^{-}\right)$similar to the one in (5.9) where utility is fixed 0 at $0 \in X$ and 1 at $w \in X$. Secondly, we can show that the functions $\mathrm{CEU}_{\rho}^{+}\left(\mathrm{CEU}_{\rho}^{-}\right)$are restrictions of a general CEU-function $\mathrm{CEU}^{+}\left(\mathrm{CEU}^{-}\right)$, with unique multilinear utility and unique capacity, representing $\succcurlyeq$ on $X_{+}^{n}\left(X_{-}^{n}\right)$. Third, we can derive $\mathrm{CPT}$ for $\succcurlyeq$ on $X^{n}$. Finally, we can prove the uniqueness results.

This completes the proof of Theorem 5.4.

Proof of Theorem 5.5. That Statement (i) implies (ii) is immediate. The proof of (i) from (ii) is analogous to that in the proof of Theorem 5.4. The difference is that for the preference relation utility independence for attribute sets is replaced by mutual utility independence. In Lemma 5.11 this is not yet relevant, therefore Lemma 5.11 holds here. Utility independence for attribute sets was relevant in Lemma 5.12. Considering mutual utility independence instead, Lemma 5.12 remains valid if we replace " $U_{i d}^{z}$ is a multilinear 
utility function" by " $U$ id is additive/multiplicative utility function". Then in the proof we have to use Theorem 4 of Miyamoto \& Wakker (1996) instead of their Theorem 5. Other than that, the proof can entirely be adapted with a additive/multiplicative utility $U_{\text {id }}^{z}$ instead of a multilinear one.

Similarly, Lemma 5.13 remains valid with additive/multiplicative utility instead of multilinear utility. Further, the analysis following the proof of Lemma 5.13 can entirely be repeated here, concluding Statement (i) in Theorem 5.5. This completes the proof. 


\section{Chapter 6}

\section{A Class of Methods for Evaluating Multiattribute Utilities for Health}

\section{States}

\subsection{Introduction}

In an empirical study that was conducted among hearing impaired individuals numerical information regarding their health, or rather, hearing states was collected. These hearing states were composed of levels ('bad', 'reasonable' or 'good') of five different attributes (ability to listen to the news on the radio, to follow a conversation at a party, and the like). After some numerical operations a collection of numbers between zero and one for all $\left(3^{5}=243\right)$ health states was obtained. The number zero corresponds to the state where all attributes are at their minimal level, and the number one to the combination of all attributes at the maximal level.

In this situation one question that arises is the following: What is the contribution of each of the $(3 \cdot 5=15)$ levels to reaching the perfect, maximal hearing state?

An answer to this question, i.e. a method to evaluate such contributions, can be of great practical value. It tells us on which level of which attribute we should concentrate a possible medical intervention program if we wish to achieve maximal perceived improvement in a person's health (i.c. hearing) statc, given a limited financial budget. To be more specific, suppose such a method results in numerical values for the contributions of the different

\footnotetext{
${ }^{0}$ The results in this chapter were first formulated in Peters \& Zank (1999).
} 
attribute levels to health improvement, over a population of hearing impaired persons. Suppose moreover that data on the costs of specific interventions are available. Then one may be able to calculate the most cost effective improvement, and direct the intervention program to that improvement. But even if such detailed cost data are not available or very specific interventions not feasible, then numerical values for the contributions of the attribute levels that rnake up the individual health states, can still support medical decision making in a qualitative way.

The class of methods that will be developed in this chapter may in fact be applied to a wide range of multiattribute numerical data. Therefore, an abstract notation is used to describe situations, states, attributes and levels. However, sometimes health states are used to illustrate the concepts and assumptions.

Important inspiration comes from some concepts and results in the theory of cooperative games. In particular, from the work of Dubey (1975) and Einy (1988) because they (also) restrict attention to classes of simple and/or monotonic games; and from Weber (1988) for the study of the concept of marginalism in Section 4 below. More detailed references will be given at the appropriate places. This work is also formally related to the work on multichoice games (Hsiao and Raghavan, 1993, and Faigle and Kern, 1992), and the methods developed in this chapter could be applied to such games as well.

In Section 6.2 of this chapter the main concepts that play a role in the sequel, are introduced. The class of contribution methods that is considered is characterized in Section 6.3. This means that a justification is provided on the basis of more elementary assumptions. These assumptions implicitly entail the idea of marginal contributions, a point that is elaborated in Section 6.4. In Section 6.5, one specific method, the so called egalitarian valuation, is studied in detail. Section 6.6 concludes the main part of the chapter. In the Appendix all mathematical proofs are collected.

\subsection{Main Concepts}

Throughout the collection of attributes $N=\{1,2, \ldots, n\}$ and the collection of levels $M=$ $\{0,1,2, \ldots, m\}(m \geqslant 1)$ are fixed. A state is a map $\omega: N \rightarrow M$. So a state attaches a level to each attribute. Observe that we assume implicitly that each attribute has the same number of levels, namely $m+1$. This is just for convenience: also without this assumption an accurate description of the 'right' levels may be a tricky problem in practical situations. The set of all states is denoted by $\Omega$. In mathematical notation: $\Omega=M^{N}$. 
On the set $\Omega$ there is a natural ordering. Identifying $\omega \in \Omega$ with the vector $(\omega(1), \omega(2), \ldots, \omega(n))$ of the levels of the $n$ attributes, we write $\omega \geqslant \omega^{\prime}$ for $\omega, \omega^{\prime} \in \Omega$ if this inequality holds for each coordinate, i.e. if $\omega(i) \geqslant \omega^{\prime}(i)$ for all $i \in N$. This means that the level of every attribute is at least as high in $\omega$ as it is in $\omega^{\prime}$.

A situation $h$ is a collection of numerical values for all possible $\left((m+1)^{n}\right)$ states satisfying a few additional properties. Formally, it is a map $h: \Omega \rightarrow \mathbb{R}$ satisfying

(i) $h(0,0, \ldots, 0)=0$,

(ii) for all $\omega, \omega^{\prime} \in \Omega, \omega \geqslant \omega^{\prime}$ implies $h(\omega) \geqslant h\left(\omega^{\prime}\right)$.

Property (i) normalizes the value of the worst possible state to zero. This is in accordance with common practice in health sciencel. Property (ii) states that the assigned values should be monotonic; this is certainly a natural assumption if utilities for health states are concerned. Let $H$ denote the collection of all situations (in symbols: $H=\mathbb{R}^{\Omega}$ ). Observe that (i) and (ii) imply that $h(\omega) \geqslant 0$ for all $h \in H$ and $\omega \in \Omega$.

We wish to say something about the impact or importance of each level of each attribute for any given situation $h \in H$. Formally this means that we will study maps $\varphi: H \rightarrow$ $I^{n \times(m+1)}$, i.e., maps that assign to each situation a collection of numbers, one for each level of each attribute. For a situation $h$, an attribute $i$ and a level $j$, the number $\varphi_{i, j}(h)$ expresses the importance or contribution of level $j$ of attribute $i$ in situation $h$. Specifically, when health situations are concerned, this number $\varphi_{i, j}(h)$ should tell us something about what it is worth to reach level $j$ of attribute $i$ on the way to the perfect health state $(m, m, \ldots, m)$.

In order to be justified in attaching this interpretation to such a map $\varphi$ we need to make some assumptions. The first assumption says that the values assigned by $\varphi$ should indeed be a distribution of the value of the perfect state.

Distribution (D) For all $h \in H, \sum_{i \in N, j \in M} \varphi_{i, j}(h)=h\left(m, m_{1} \ldots, m\right)$.

The next assumption captures to some extent the idea of 'contribution'. Suppose that in a certain situation lowering level $j$ of attribute $i$ to level $j-1$ never makes any difference: the value of $h$ does not change. Then it is only natural to attach a value of zero to the

\footnotetext{
${ }^{1}$ In cooperative game theory this corresponds to the convention of setting the worth of the empty coalition equal to 0 .
} 
original level $j$. Formally, this leads to the following condition.

Zero Contribution (ZC) For all $h \in H, i \in N, j \in M$, if $h(\omega)=h\left(\omega^{\prime}\right)$ for all $\omega, \omega^{\prime} \in \Omega$ with $\omega(i)=j, \omega^{\prime}(i)=j-1$ and $\omega(k)=\omega^{\prime}(k)$ for all $k \in N, k \neq i$, then $\varphi_{i, j}(h)=0$.

Observe that, trivially, this condition implies that $\varphi_{i, 0}(h)=0$ for all $h \in H$ and $i \in N$.

In health situations the value of the perfect state is often scaled to one. If this is not the case, we would want our contribution measure $\varphi$ to be independent of the value of the perfect state, so that we can always scale it to one. This is expressed by the following property, where we use the notations $(a h)(\omega):=a h(\omega)$ and $a \varphi(h):=\left(a \varphi_{i, j}(h)\right)_{i, j}$ for $h \in H$ and $a \in \mathbb{R} R_{+}$.

Homogeneity (H) For all $h \in H$ and all $a \in \mathbb{R}$ with $a \geqslant 0$ we have $\varphi(a h)=a \varphi(h)$.

In many practical situations we obtain the numerical values of $h$ by measurement. In health situations for instance, these numbers are often obtained by interviewing individuals. Clearly, individuals or interviewers may make mistakes and, moreover, sharp-cut individual values may simply fail to exist. Suppose now that we have obtained two sets of values $h$ and $h^{\prime}$ of the same population. An obvious way to estimate the 'true' values would be to take the (state-wise) average of $h$ and $h^{\prime}$. A corresponding natural condition on $\varphi$ would be to require that it should not make a difference whether $\varphi$ is applied to this average or whether the average of the $\varphi$-values is calculated. Moreover, the former calculation is generally speaking easier to perform than the latter. In symbols, we would require

$$
\varphi\left(\frac{h+h^{\prime}}{2}\right)=\frac{\varphi(h)+\varphi\left(h^{\prime}\right)}{2} .
$$

Under homogeneity, this implies

$$
\varphi\left(h+h^{\prime}\right)=\varphi(h)+\varphi\left(h^{\prime}\right) .
$$

A consequence of this condition would be that it does not make a difference whether we calculate the $\varphi$-values either for $h$ and $h^{\prime}$ and then add, or for the new situations arising from taking (state-wise) minima of $h$ and $h^{\prime}$ and (state-wise) maxima of $h$ and $h^{\prime}$, respectively, and then add. It is this weaker condition that will be imposed. ${ }^{2}$ In order to formalize this, define for $h, h^{\prime} \in H$ the new situations $h \vee h^{\prime}$ and $h \wedge h^{\prime}$ by $h \vee h^{\prime}(\omega):=\max \left\{h(\omega), h^{\prime}(\omega)\right\}$

\footnotetext{
${ }^{2}$ The reason for this is partly technical. It allows us to use representations of situations on the basis of
} 
and $h \wedge h^{\prime}(\omega):=\min \left\{h(\omega), h^{\prime}(\omega)\right\}$ for all $\omega \in \Omega$.

Transfer Property (TP) For all $h, h^{\prime} \in H$,

$$
\varphi\left(h \vee h^{\prime}\right)+\varphi\left(h \wedge h^{\prime}\right)=\varphi(h)+\varphi\left(h^{\prime}\right) .
$$

The name Transfer Property (cf. Dubey, 1975) comes from the observation that $h \vee h^{\prime}$ and $h \wedge h^{\prime}$ arise from $h$ and $h^{\prime}$ by transferring maximal and minimal values between these two.

A map $\varphi: H \rightarrow \mathbb{R}^{n \times(m+1)}$ satisfying the Transfer Property is called a valuation. ${ }^{3}$ The remainder of the chapter is concerned with studying valuations satisfying the properties of Distribution, Homogeneity, and Zero Contribution.

\subsection{A Characterization of Valuations}

In order to characterize valuations we consider their values on a specific subclass of situations. This subclass should be large enough so as to determine a valuation on the whole class $H$. It should consist of situations that are transparent enough so as to reveal the implicit assumptions that are made if we choose values for a valuation in those situations. A subclass of situations satisfying both requirements is the class of so called simple situations. For every $\omega \in \Omega, \omega \neq(0,0, \ldots, 0)$ define the simple situation $h_{\omega}$ by

$$
h_{\omega}\left(w^{\prime}\right)= \begin{cases}1 & \text { if } \omega^{\prime} \geqslant \omega \\ 0 & \text { otherwise }\end{cases}
$$

Such a simple situation is characterized by a fixed state that has value one. All states with at least one level below this given state have zero value, all other states have value equal to one. The collection of all simple situations is denoted by $S .{ }^{4}$

An individual reporting a health situation of the form $h_{\omega}$ would probably be a rare event, because such an individual would only be interested in reaching a certain minimal level in any attribute. The point, however, is that these situations seem very well suited to fix a valuation.

so called simple situations, using only positive coefficients. See the Appendix for the details, in particular Lemma 6.9.

${ }^{3}$ The term valuation comes from the theory of lattices, see for instance Topkis (1998). See also Einy (1988, page 5).

${ }^{4}$ Simple situations correspond to unanimity games in cooperative game theory. 
It turns out that the Transfer Property of a valuation is sufficient to extend it uniquely from simple situations to all situations if we additionally impose Homogeneity. The proof of this result, like all other proofs, is given in the Appendix.

Theorem 6.1 Let $\varphi^{\prime}: S \rightarrow \mathbb{R}^{n \times(m+1)}$. Then there is a unique homogeneous valuation $\varphi$ that coincides with $\varphi^{\prime}$ on $S$.

This theorem is of central importance. It tells us that, in order to define a homogeneous valuation, it is sufficient to choose its values on the class of simple situations. The next theorem tells us how this choice is restricted by the conditions of Distribution and Zero Contribution and, moreover, that it is possible to do so.

THEOREM 6.2 Let $\varphi$ be a homogeneous valuation. Then $\varphi$ satisfies Zero Contribution if, and only if, for every nonzero $\omega \in \Omega$, we have

$$
\text { (i) } \varphi_{1, j}\left(h_{\omega}\right)=0 \text { for all } i \in N \text { and } j \in M \text { with } \omega(i) \neq j \text { or } j=0 \text {, }
$$

and $\varphi$ satisfies Distribution if, and only if, for every nonzero $\omega \in \Omega$, we have

(ii) $\sum_{i \in N, j \in M} \varphi_{i, j}\left(h_{\omega}\right)=1$

By combining these two Theorems we see that a valuation with the three additional properties of Homogeneity, Distribution, and Zero Contribution is completely determined by distributing, for every nonzero state, the total of one over the nonzero levels of that state. There are obviously still many ways to do this. A common requirement, should be that the values assigned by a valuation are independent of the order in which the attributes are placed. To formalize this we need some notation. For a permutation $\sigma$ of $N$, a state $\omega \in \Omega$ and a situation $h \in H$ define $\sigma \omega \in \Omega$ by $\sigma \omega(\sigma(i)):=\omega(i)$ for every $i \in N$ and $\sigma h \in H$ by $\sigma h\left(\sigma \omega^{\prime}\right):=h\left(\omega^{\prime}\right)$ for every $\omega^{\prime} \in \Omega$. So $\sigma h$ arises from $h$ by interchanging attributes according to $\sigma$.

Attribute Symmetry (AS) For every permutation $\sigma$ of $N$, every attribute $i \in N$, every level $j \in M$ and every situation $h \in H, \varphi_{i, j}(h)=\varphi_{\sigma(i), j}(\sigma h)$.

In order to obtain an attribute symmetric homogeneous valuation it is sufficient to satisfy this property on simple situations, as the following theorem shows. 
THEOREM 6.3 Let $\varphi$ be a homogeneous valuation. Then $\varphi$ is attribute symmetric if, and only if, for every permutation or of $N$, every attribute $i \in N$, every level $j \in M$ and every $\omega \in \Omega, \varphi_{i, j}\left(h_{\omega}\right)=\varphi_{\sigma(i), j}\left(h_{\sigma \omega}\right)$.

In the remainder of this section we focus on some examples of how values on simple situations can be chosen consistently with Zero Contribution, Distribution, and Attribute Symmetry.

In the first and in this chapter most important example we argue as follows. For a nonzero state $\omega$ each of the nonzero levels of $\omega$ has to be reached in $h_{\omega}$ in order to improve from zero to one. Thus, each of these levels is equally important and should be assigned the same value by $\varphi$. Another argument in favor of this approach is that in practical situations we would want our valuation to be robust against imperfections in the description of levels - think in particular of health situations. Thus, it should not matter too much whether a particular level of an attribute ends up as a high or a low level. A formal description is given below, in Definition 6.4. Moreover, the resulting egalitarian valuation is studied extensively in Section 6.5 .

A second more or less opposite example is obtained if we take into account the number of states for which a particular level of an attribute is pivotal. To be specific, suppose $\omega \in \Omega$ we have $\omega(i)>0$. Then, we could require $\varphi_{i, \omega(i)}\left(h_{\omega}\right)$ to be proportional to the number of states $\omega^{\prime} \geqslant \omega$ with $\omega^{\prime}(i)=\omega(i)$ because these are exactly the states whose value in the simple situation $h_{\omega}$ would decrease from one to zero if the level $\omega(i)$ is decreased by one to level $\omega(i)-1$. If we think of applying this idea to health states in a population of individuals, the implicit assumption is that all states are equally likely to occur. Here, we will restrict our attention to this symmetric case. ${ }^{5}$ A little reflection shows that this method favors high levels because the number of states for which these levels are pivotal is relatively bigger.

As an intermediate example one can choose the contribution of each level $\omega(i)$ in a simple situation $h_{\omega}$ to be proportional to the height of the level.

Formally, we have the following three valuations.

\section{DEFINITION 6.4}

- The egalitarian valuation is the homogeneous valuation, denoted by $\varepsilon$, with for all

\footnotetext{
${ }^{5}$ For the case of asymmetric distributions the method should be adapted.
} 
nonzero $\omega \in \Omega$, all $i \in N$ and all $j \in M$ :

$$
\varepsilon_{i, j}\left(h_{\omega}\right)= \begin{cases}\frac{1}{n(\omega)} & \text { if } \omega(i)=j \text { and } j \neq 0 \\ 0 & \text { otherwise, }\end{cases}
$$

where $n(\omega)$ denotes the number of nonzero levels of $\omega$.

- The pivotal valuation is the homogeneous valuation, denoted by $\rho$, with for all nonzero $\omega \in \Omega$, all $i \in N$ and all $j \in M$ :

$$
\rho_{i, j}\left(h_{\omega}\right)= \begin{cases}\frac{\Pi_{k \in N-\{i\}}(m-\omega(k)+1)}{\sum_{i \in N} \Pi_{k \in N-(\ell)}(m-\omega(k)+1)} & \text { if } \omega(i)=j \text { and } j \neq 0 \\ 0 & \text { otherwise. }\end{cases}
$$

- The proportional valuation is the homogeneous valuation, denoted by $\pi$, with for all nonzero $\omega \in \Omega$, all $i \in N$ and all $j \in M$ :

$$
\pi_{i, j}\left(h_{\omega}\right)= \begin{cases}\frac{\omega(i)}{\sum_{k=1}^{n} \omega(k)} & \text { if } \omega(i)=j \\ 0 & \text { otherwise. }\end{cases}
$$

This definition describes the examples above formally for simple situations. The general form is given in Lemma 6.12 in the Appendix.

In Section 6.5 we will focus on the egalitarian valuation. The reason is that, particularly in health situations, there are good reasons (mentioned above) to treat levels in a symmetric way. Moreover, we do not see any compelling reason for an asymmetric treatment. The pivotal valuation for instance is based on the assumption that every way to reach a certain health state is equally probable, but for health situations this does not seem likely. ${ }^{6}$ In Section 6.5 we will actually show that imposing a condition of "Level Symmetry" singles out the egalitarian valuation.

In the next section we show that every hornogeneous valuation having the Zero Contribution property satisfies a property of marginalism: its values depend only on marginal contributions. This leads to alternative formulations of such valuations, and in particular of the egalitarian, pivotal, and proportional valuations.

\subsection{Marginalism}

As explained in the Introduction the idea underlying valuations is that they should represent the contribution of each level to reaching the "perfect" state. The following property

\footnotetext{
${ }^{6}$ In a game-theoretic context such an argument may be more convincing. See Faigle and Kern (1992) who develop a method based on the number of paths by which a certain 'state' can be reached.
} 
gives this idea a precise meaning. It says that the value assigned to each level of each attribute should only depend on the marginal contributions of that level and not on any other aspects of a situation. ${ }^{7}$

Marginalism (M) For all $h, h^{\prime} \in H, i \in N, j \in M$, if $h(\omega)-h\left(\omega^{\prime}\right)=h^{\prime}(\omega)-h^{\prime}\left(\omega^{\prime}\right)$ for all $\omega, \omega^{\prime} \in \Omega$ with $\omega(k)=\omega^{\prime}(k)$ for all $k \neq i, \omega(i)=j$, and $\omega^{\prime}(i)=j-1$, then $\varphi_{i, j}(h)=\varphi_{i, j}\left(h^{\prime}\right)$.

Zero Contribution is in fact a weak form of Marginalism. It turns out that Zero Contribution implies Marginalism for a homogeneous valuation and that, moreover, such a valuation is an additive function of marginal contributions. For $\omega \in \Omega$ denote by $\omega+e_{i}$ [resp. $\omega-e_{i}$ ] the state arising by increasing [resp. decreasing] the level of attribute $i$ by one (if possible).

THEOREM 6.5 Let $\varphi$ be a homogeneous valuation satisfying Zero Contribution. Then $\varphi$ satisfies Marginalism and for every $i \in N$ and $j \in M, j \neq 0$ there is a collection of real numbers $\left\{p_{\omega}: \omega \in \Omega\right.$ with $\left.\omega(i)=j-1\right\}$ such that

$$
\varphi_{i, j}(h)=\sum_{\omega \in \Omega:} p_{\omega(i)=j-1}\left[h\left(\omega+e_{i}\right)-h(\omega)\right]
$$

for all $h \in H$

If, additionally, $\varphi$ satisfies Distribution then these numbers $p_{\omega}$ sum to one.

This theorem implies that also the egalitarian valuation $\varepsilon$, the pivotal valuation $\rho$, and the proportional valuation $\pi$ have an additive representation on the basis of marginal contributions. The result can be seen as the analogon of similar results for cooperative games in Weber (1988).

For attribute symmetric valuations we have the following result. To avoid confusion we will sometimes use a notation like $p_{w}^{i}$ instead of $p_{w}$ as was used in Theorem 6.5.

Theorem 6.6 Let $\varphi$ be a homogeneous valuation satisfying Zeto Contribution and Attribute Symmetry, and let for $i \in N$ and $0 \neq j \in M$ the weights $p_{\omega}$ be as determined in Theorem 6.5. Let $\sigma$ be a permutation of $N$. Then for every $\omega$ with $\omega(i)=j-1$ we have $p_{\omega}^{i}=p_{\sigma \omega}^{\sigma(i)}$.

\footnotetext{
${ }^{7}$ In Young (1985) this idea was exploited to characterize the Shapley value (Shapley, 1953) for cooperative games.
} 
Theorem 6.6 implies that under Attribute Symmetry the weights $p_{\omega}$ depend only on the level and not on the particular attribute to which that level is assigned. Again, this theorem applies to the egalitarian, pivotal, and proportional valuations.

\subsection{The Egalitarian Valuation}

In this section we focus on one particular method, the egalitarian valuation, as described in Definition 6.4. The main result will be an axiomatic characterization of this valuation.

Let $i \in N$ be an attribute, $h \in H$ a situation, and let $l, l^{\prime} \in M \backslash\{0\}$ be distinct levels. We call $l$ and $l^{\prime}$ symmetric in attribute $i$ for the situation $h$ if for all $\omega, \omega^{\prime} \in \Omega$ with $\omega(i)=l, \omega^{\prime}(i)=l^{\prime}$ and $\omega(k)=\omega^{\prime}(k)$ for $k \neq i$ the following holds:

$$
h(\omega)-h\left(\omega-e_{i}\right)=h\left(\omega^{\prime}\right)-h\left(\omega^{\prime}-e_{i}\right) .
$$

In words, two levels of an attribute are symmetric if decreasing them by one has the same effect on the value of a state. It seems only natural that to such levels a method that is intended to measure contributions should assign the same value. Formally, this leads to the following condition for a valuation $\varphi$ on $H$.

Level Symmetry (LS) For any $h \in H, i \in N$ we have $\varphi_{i, l}(h)=\varphi_{i, l}(h)$ for all $l, l^{\prime} \in M \backslash\{0\}$ that are symmetric in attribute $i$ for the situation $h$.

We now have the following characterization of the egalitarian valuation.

THEOREM $6.7 \varphi: H \rightarrow \mathbb{R}^{n \times(m+1)}$ is a homogeneous valuation satisfying Zero Contribution and Distribution, Attribute Symmetry and Level Symmetry if, and only if, $\varphi$ is the egalitarian valuation.

Observe that the pivotal and proportional valuations satisfy all the conditions in this theorem except Level Symmetry. ${ }^{8}$

We conclude this section with some remarks on computation. One way to compute the egalitarian valuation (and for that matter, the pivotal and proportional valuations) for a given situation $h \in H$ is to first cormpute the representation of $h$ on simple situations $h_{\omega}$

${ }^{8}$ If we take $m=1$ and drop Level Symmetry, which has no bite anyway in that case, then basically the Theorem characterizes the Shapley value (Shapley, 1953). Therefore the class of methods satisfying all the conditions in the Theorem but not necessarily Level Symmetry, extends the Shapley value. 
(see Lemma 6.9 and formulas (6.1) and (6.2)) and then compute the valuation according to Lemma 6.12. Alternatively, one may determine the weights $p_{\omega}$ as in Theorem 6.5 and then use the expression in Theorem 6.5 to calculate the egalitarian (and in principle also the pivotal and proportional) evaluation of $h$. These weights $p_{\omega}$ are derived in Lemmas 6.7 and 6.13: They are related to the values assigned to specific situations by the linear extension of a valuation to the class of nonmonotonic situations. For the egalitarian valuation these values can be determined from Theorem 6.8 describing the values assigned by the egalitarian valuation to the situations $\kappa_{\omega} \in \vec{H}$, where $\vec{H}$ is the set of situations with the monotonicity condition dropped. These values are of interest because they give the weights $p_{\omega}$ in Theorem 6.5 through the relation $p_{\omega}=-\varphi_{i, j}\left(\kappa_{\omega}\right)$ (cf. Lemmas 6.7 and 6.13 and their proofs).

Henceforth, assume $m \geqslant 2, n \geqslant 2$ to avoid trivial or special cases. Let. $k \in\{0, \ldots, n-1\}$ and $j \in\{1, \ldots, m\}$. Define the state $\omega_{k, j} \in \Omega$ as follows

$$
\omega_{k, j}:=(\underbrace{0, \ldots, 0}_{k}, j \cdot \underbrace{m, \ldots, m}_{n-k-1}) .
$$

The following theorem is formulated for states $\omega_{k, j}$. Similarly, the result holds for states $\sigma\left(\omega_{k, j}\right)$, for any permutation $\sigma$ of $N$, because the egalitarian valuation satisfies Attribute Symmetry. However, to avoid tedious notation, in what follows we suppress $\sigma$.

THEOREM 6.8 Let $\varepsilon: \widetilde{H} \rightarrow \mathbb{R}^{n \times(m+1)}$ be the egalitarian valuation. Then, the follouing hold for $\kappa_{\omega_{k, j}}$ :

(i) If $k=0$ and $j=m$, then

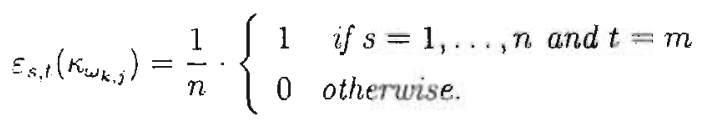

(ii) If $k>0$ and $j=m$, then

$$
\varepsilon_{s, t}\left(\kappa_{\omega_{k, j}}\right)=-\frac{(k-1) !}{\prod_{i-n}^{k}(n-r)} \cdot \begin{cases}k-n & \text { for } s=1, \ldots, k \text { and } t=1 \\ k & \text { for } s=k+1, \ldots, n \text { and } t=m \\ 0 & \text { otherwise. }\end{cases}
$$

(iii) If $j \in\{1, \ldots, m-1\}$, then

$$
\varepsilon_{s, t}\left(\kappa_{\omega_{k, j}}\right)=\frac{k !}{\prod_{r=1 !}^{k}(n-r)} \cdot \begin{cases}1 & \text { for } s=k+1 \text { and } t=j \\ -1 & \text { for } s=k+1 \text { and } t=j+1 \\ 0 & \text { otherwise. }\end{cases}
$$


(iv) Finally, if a state $\omega$ has at least two levels in $\{1, \ldots, m-1\}$, then $\varepsilon_{s, t}\left(\kappa_{\omega}\right)=0$ for all $s=1, \ldots, n$ and $t=1, \ldots, m$.

As observed before (see footnote 8) every homogeneous valuation satisfying Zero Contribution, Distribution, and Attribute Symmetry extends the Shapley value of cooperative game theory (Shapley, 1953). The egalitarian valuation does this in a even more special way, as we will demonstrate now. Let $h \in H$ be any situation. Theorem 6.5 implies:

$$
\varepsilon_{i, j}(h)=\sum_{\omega \in \Omega: \omega(i)=j-1} p_{\omega}\left[h\left(\omega+e_{i}\right)-h(\omega)\right] .
$$

According to the last two statements in Theorem 6.8 , the weight $p_{\omega}\left(=-\varepsilon_{i, j}\left(\kappa_{\omega}\right)\right)$ is equal to 0 if there exists an attribute $s \neq i$ for which $\omega(s) \notin\{0, m\}$. This follows from Statement (iii) if $j=1$, and from Statement (iv) if $j>1$. Consequently, the egalitarian valuation takes marginal contributions in attribute $i$ into account only when the levels of all other attributes are either 0 or $m$, ignoring contributions elsewhere. Hence, in the formula above summation over the set $\{\omega \in \Omega: \omega(i)=j-1\}$ can be reduced to summation over the set $C:=\{\omega \in \Omega: \omega(i)=j-1, \omega(s) \in\{0, m\}$ for all $s \neq i\}$.

Let us take a closer look at the states in this set. Obviously, each $\omega \in C$ can be written as $\sigma\left(\omega_{k, j-1}\right)$ for a permutation $\sigma$ of $N$, and a state $\omega_{k, j-1}$ as defined before Theorem 6.8. By Attribute Symmetry (Theorem 6.6) we know that $p_{\sigma\left(\omega_{k, j-1}\right)}^{i}=p_{\omega_{k, j-1}}^{k+1}$, and by the definition of the weights $p_{-}$(see the proofs of Lemmas 6.7 and 6.13 ) we have

$$
p_{\omega_{k, j-1}}=-\varepsilon_{k+1, j}\left(\kappa_{\omega_{k, j-1}}\right) .
$$

If $j \in\{2, \ldots, m\}$ Statement (iii) of Theorem 6.8 implies

$$
p_{\omega_{k, j-1}}=-\varepsilon_{k+i, j}\left(\kappa_{\omega_{k, j-1}}\right)=\frac{k !}{\prod_{r=0}^{k}(n-r)},
$$

which can be rewritten as

$$
p_{\omega_{k, j-1}}=\frac{k !(n-1-k) !}{n !} .
$$

If $j=1$, Statement (ii) of Theorem 6.8 implies

$$
p_{\omega_{k, a}}=-\varepsilon_{k+1,1}\left(\kappa_{\omega_{k, 0}}\right)=-\varepsilon_{k+1,1}\left(\kappa_{\omega_{k+1, m}}\right)=-\frac{k !(k+1-n)}{\prod_{r=0}^{k}(n-r)},
$$


which after rearranging yields

$$
p_{\omega_{k, 0}}=\frac{k !(n-1-k) !}{n !}
$$

Hence, for all $j=1, \ldots, m$ the weights $p_{\omega_{k, j-1}}=\frac{k !(n-1-k) !}{n !}$ depend only on the number of levels that are equal to 0 .

To compare this with the Shapley value in cooperative game theory note that attributes can be viewed as players, and consider the marginal contribution $h\left(\omega_{k, j}\right)-h\left(\omega_{k, j-1}\right)$. This contribution can be seen as the result of player $k+1$ increasing his level from $j-1$ to $j$ in a coalition consisting of $n-(k+1)$ players already there at full strength (that is, players $k+2, \ldots, n$ at their maximum level $m$ ) and the remaining players not (yet) there (that is, players $1, \ldots, k$ at level 0 ). In the egalitarian valuation this contribution is weighted by $p_{\omega_{k, j-1}}=\frac{k !(n-1-k) !}{n !}$, which is exactly the coefficient in the Shapley value that weights the contribution of a player joining a coalition of size $k$ or of size $n-k-1$ in random order (See for instance Weber, 1988, page 118).

\subsection{Concluding Remarks}

In this chapter a class of methods to evaluate the contributions of different attribute levels in (health) situations is developed. This class is characterized by properties that are quite natural in this context: Distribution, Zero Contribution, Homogeneity, and the Transfer Property. It turns out that these properties imply the property of Marginalism, again a natural condition in this framework. By imposing the symmetry conditions Attribute Symmetry and Level Symmetry we have obtained a characterization of the egalitarian valuation.

The focus of this chapter is mainly theoretical, and indeed there is scope for further theoretical development, like characterizations of alternative valuations. From the practical point of view, an application to the health situation of hearing impaired individuals as mentioned in the Introduction to this chapter will be described in a forthcoming paper.

\subsection{Appendix: Proofs to Chapter 6}

For $h \in H$ and $\alpha \in \mathbb{R}_{+}$(the set of nonnegative real numbers) denote by $\Omega(\alpha, h)$ the set of minimal states with value $c x$ under $h$, i.e.

$$
\Omega(\alpha, h):=\left\{\omega \in \Omega: h(\omega)=\alpha \text { and } h\left(\omega^{\prime}\right)<\alpha \text { for all } \omega^{\prime} \in \Omega \text { with } \omega \geqslant \omega^{\prime}, \omega \neq \omega^{\prime}\right\} .
$$


As before, let $\vee$ denote the maximum operator.

LEMMA 6.9 Let $h \in H$, with range $h(\Omega)=\left\{0, \alpha_{1}, \ldots, \alpha_{r}\right\}$. Then

$$
h=\bigvee_{i=1}^{r} \bigvee_{\omega \in \Omega\left(\alpha_{i}, h\right)} \alpha_{i} h_{\omega}
$$

Proof. Take $0 \neq \omega^{\prime} \in \Omega$ arbitrary, and suppose $h\left(\omega^{\prime}\right)=\alpha_{i}$ for some $i \in\{1, \ldots, r\}$. Note that, by the monotonicity of $h$ (property (ii) in the definition of a situation) and the assumption that the zero state always has value zero (property (i)), the range of $h$ can only contain nonnegative numbers. For the right hand side of (6.1) we obtain

$$
\bigvee_{i=1}^{r} \bigvee_{\omega \in \Omega\left(\alpha_{i}, h\right)} \alpha_{i} h_{\omega}\left(\omega^{\prime}\right) \geqslant \alpha_{i}
$$

Suppose this inequality were strict. Then there would be a $k \in\{1, \ldots, r\}$ and an $\omega^{\prime \prime} \in \Omega\left(\alpha_{k}, h\right)$ with $\omega^{\prime \prime} \leqslant \omega^{\prime}$ and $\alpha_{k} h_{\omega^{\prime \prime}}\left(\omega^{\prime}\right)>\alpha_{i}$, in particular $h\left(\omega^{\prime \prime}\right)=\alpha_{k}>\alpha_{i}=h\left(\omega^{\prime}\right)$. Because $\omega^{\prime \prime} \leqslant \omega^{\prime}$ this contradicts the monotonicity of $h$.

It can be shown that the representation in (6.1) of $h \in H$ is in fact unique among representations of this form. This fact, however, will not be used in the sequel. For our purposes, it is sufficient to note that every $h \in H$ has a representation of the form

$$
h=\bigvee_{i \in I} \alpha_{i} h_{\omega^{\prime}}
$$

on the collection of simple situations, where $I$ is some finite index set.

As before, let $\wedge$ denote the minimum operator. The following lemma expresses the valuation of a maximum of a collection of situations in terms of their minima.

Lemma 6.10 Let $\varphi$ be a valuation and let $h_{1}, h_{2}, \ldots, h_{\tau} \in H$. Then $\bigvee_{i=1}^{r} h_{i} \in H$ and

$$
\begin{aligned}
\varphi\left(V_{i=1}^{r} h_{i}\right)= & \sum_{i=1}^{r} \varphi\left(h_{i}\right)-\sum_{i<j}^{r} \varphi\left(h_{i} \wedge h_{j}\right) \\
& +\sum_{i<j<\ell}^{r} \varphi\left(h_{i} \wedge h_{j} \wedge h_{\ell}\right) \\
& \vdots \\
& +(-1)^{r+1} \varphi\left(\bigwedge_{i=1}^{r} h_{i}\right) .
\end{aligned}
$$

Proof. Straightforward, using the Transfer Property, by induction on $r$.

Also the proof of the following lemma is straightforward and left to the reader (cf. also Einy, 1988, Lemma 11). 
Lemma 6.11 Let $\alpha, \beta \in \mathbb{R}_{+}$and $\omega, \omega^{2} \in \Omega$. Then

$$
\alpha h_{\omega} \wedge \beta h_{\omega^{\prime}}=(\alpha \wedge \beta) h_{\omega \vee \omega^{\prime}}
$$

in particular, $\alpha h_{\omega} \wedge \beta h_{\omega^{\prime}}$ is of the form $\gamma h_{\omega^{\prime \prime}}$ for some $\gamma \in \mathbb{R}_{+}$and $\omega^{\prime \prime} \in \Omega$.

The next lemma gives an expression for homogeneous valuations on $H$ with the aid of simple situations in $S \subset H$. It follows from Lernmas 6.10 and 6.11 .

LemMa 6.12 Let $\varphi$ be a homogeneous valuation, and let $h \in H$ have the representation (6.2) with $I=\{1, \ldots, r\}$. Then

$$
\begin{aligned}
\varphi(h)= & \sum_{i=1}^{r} \alpha_{i} \varphi\left(h_{\omega^{l}}\right)-\sum_{i, j \in I, i<j}\left(\alpha_{i} \wedge \alpha_{j}\right) \varphi\left(h_{\omega^{2} \vee \omega^{3}}\right) \\
& +\sum_{i, j, \ell \in I, i<j<\ell}\left(\alpha_{i} \wedge \alpha_{j} \wedge \alpha_{\ell}\right) \varphi\left(h_{\omega^{i} \vee \omega^{j} \vee \omega^{\ell}}\right) \\
& \vdots \\
& +(-1)^{r+1} \wedge_{i=1}^{r} \alpha_{i} \varphi\left(h_{\vee_{i \in I}}\right) .
\end{aligned}
$$

Proof of Theorem 6.1. Follows from Lemmas 6.12 and 6.11.

Proof of Theorem 6.2. The only-if part is left to the reader.

Let $\varphi$ be a homogeneous valuation satisfying (i) and (ii) in the theorem for every nonzero $\omega \in \Omega$. We first show $Z C$ of $\varphi$. Let $i, j, h$ be as in the formulation of $Z C$. Let $h=\vee_{k \in I} \alpha_{k} h_{\alpha^{k}}$ as in (6.2). Because every $\alpha_{k}$ is positive, it follows that the condition in $Z C$ holds for every $h_{\omega^{k}}$, and hence $\varphi_{i, j}\left(h_{\omega^{k}}\right)=0$ for every $k \in I$ by (i). Using Lemmas $6.11,6.12$, (i) and induction, it is sufficient to prove that, for all $k, \ell \in I$ and $\omega, \omega^{\prime}$ as in the definition of $Z C$ we have: $h_{\omega^{k} \omega^{l}}(\omega)-h_{\omega^{k} \mathrm{~V} \omega^{l}}\left(\omega^{l}\right)=0$. If $h_{\omega^{k} \omega^{l}}(\omega)=0$ then also $h_{\omega^{k} \vee \omega^{l}}\left(\omega^{\prime}\right)=0$ because $\omega^{\prime} \leqslant \omega$. If $h_{\omega^{k} \vee \omega^{\ell}}(\omega)=1$ then $\omega \geqslant \omega^{k}$ and $\omega \geqslant \omega^{\ell}$, hence $h_{\omega^{k}}(\omega)=1$ and $h_{\omega^{e}}(\omega)=1$. This implies that also $h_{\omega^{k}}\left(\omega^{\prime}\right)=1$ and $h_{\omega^{\prime}}\left(\omega^{\prime}\right)=1$ because both situations satisfy the condition in ZC. Therefore, $h_{\omega^{k} v_{\omega^{e}}}\left(\omega^{\prime}\right)=1$, which completes the proof.

In order to prove $D$ for $\varphi$, we use induction on $r$, with $r$ as in Lemma 6.12. For $r=1$, $D$ follows from homogeneity of $\varphi$ and (ii). For arbitrary $r$ and repeatedly applying (ii), Lemma 6.12 implies (with notation as there)

$$
\begin{aligned}
\sum_{i \in N, j \in M} \varphi_{i, j}(h)= & \sum_{k=1}^{r} \alpha_{k}-\sum_{k<\ell}^{r} \alpha_{k} \wedge \alpha_{\ell} \\
& +\sum_{k<\ell<p}^{r} \alpha_{k} \wedge \alpha_{\ell} \wedge \alpha_{p} \\
& \vdots \\
& +(-1)^{r+1} \wedge_{k=1}^{r} \alpha_{k} .
\end{aligned}
$$


To prove $D$, we have to prove that the right hand side of this equality is equal to $h(m, m, \ldots, m)$, hence to $\mathrm{V}_{k=1}^{r} \alpha_{k}$. This can be proved by induction on $r$, and is left to the reader.

Proof of Theorem 6.3. For the proof of the only-if part it is sufficient to check (left to the reader) that for every $\omega \in \Omega$ and every permutation $\sigma$ of $N$ it holds that $\sigma h_{\omega}=h_{\omega_{\omega}}$. For the if-part, it is sufficient to check that equation (6.2) implies

$$
\sigma h=\bigvee_{i \in I} \alpha_{i} h_{\sigma \omega^{i}}
$$

for every permutation $\sigma$ of $N$.

In order to prepare for the proof of Theorem 6.5 we first extend the set of situations $H$ to the larger set $\tilde{H}$ by dropping the monotonicity requirement (ii) in the definition of a situation. With the operations $\left(h+h^{\prime}\right)(\omega)=h(\omega)+h\left(\omega^{\prime}\right)$ and $(a h)(\omega)=a h(\omega)$ for all $h, h^{\prime} \in \tilde{H}, \omega \in \Omega$, and $a \in \mathbb{R}$, the set $\hat{H}$ is a linear space with the collection of simple situations as a basis. It is easily seen that the restriction of any map $\bar{\varphi}: \tilde{H} \rightarrow I R^{n \times(m+1)}$ that is linear, i.e.

$$
\bar{\varphi}\left(a h+b h^{\prime}\right)=a \tilde{\varphi}(h)+b \tilde{\varphi}\left(h^{\prime}\right) \text { for all } a, b \in \mathbb{R} \text { and } h, h^{\prime} \in \tilde{H},
$$

to $H$ is a homogeneous valuation (i.e., satisfying the transfer property) $\varphi$ on $H$, uniquely determined by its values on simple situations. Hence there is a one-to-one correspondence between homogeneous valuations on $H$ and their linear extensions on $\tilde{H}$. So we can derive properties of a homogeneous valuation $\varphi$ on $H$ from properties of its linear extension $\varphi \overline{\text { on }}$ $\bar{H}$.

As a first step we prove the following lemma.

Let $\varphi: \tilde{H} \rightarrow \mathbb{R}^{n \times(m+1)}$ be a linear map. Let $i \in N$ and $j \in M$. Then there exists a collection of constants $\left\{a_{\omega}: \omega \in \Omega\right\}$, such that for any $h \in \tilde{H}$

$$
\varphi_{i, j}(h)=\sum_{\omega \in \Omega} a_{\omega} h(\omega) .
$$

Proof. For $\omega \in \Omega, \omega \neq(0, \ldots, 0)$ we define the situation $\kappa_{\omega} \in \tilde{H}$ as follows:

$$
\kappa_{\omega}(\widetilde{\omega}):= \begin{cases}1, & \text { if } \widetilde{\omega}=\omega \\ 0, & \text { otherwise. }\end{cases}
$$


Obviously, the set $\left\{\kappa_{\omega}: \omega \in \Omega, \omega \neq(0, \ldots, 0)\right\}$ is also a basis for $\hat{H}$. Every $h \in \hat{H}$ can be uniquely written as

$$
h=\sum_{\omega \in \Omega \backslash\{(0, \cdots, 0)\}} h(\omega) r_{\omega},
$$

and linearity of $\varphi$ implies

$$
\varphi(h)=\sum_{\omega \in \Omega \backslash\{(0, \ldots, 0)\}} h(\omega) \varphi\left(\kappa_{\omega}\right)
$$

Therefore, with $a_{\omega}:=\varphi_{i, j}\left(\kappa_{\omega}\right)$ for $\omega \in \Omega \backslash\{(0, \ldots, 0)\}$ and $s_{(0, \ldots, 0)}$ arbitrary, we obtain

$$
\varphi_{i, j}(h)=\sum_{\omega \in \Omega} a_{\omega} h(\omega)
$$

This completes the proof of the lenma.

In the following two lemmas we include the properties of Zero Contribution and Distribution, the definitions of which are extended in the obvious way to maps $\varphi: \tilde{H} \rightarrow \mathbb{R}^{n \times(m+1)}$.

Lemma 6.13 Let $\varphi: \vec{H} \rightarrow \mathbb{I}^{n \times(m+1)}$ be linear and satisfy Zero Contribution. Let $i \in N$ and $j \in M \backslash\{(0, \ldots, 0)\}$. Then there exists a collection of constants $\left\{p_{\omega}: \omega \in \Omega, \omega(i)=\right.$ $j-1\}$, such that for any $h \in \tilde{H}$

$$
\varphi_{i, j}(h)=\sum_{\omega \in \Omega:} p_{\omega(i)=j-1}\left[h\left(\omega+e_{i}\right)-h(\omega)\right] .
$$

ProOf. Suppose $\omega \in \Omega$ is a state with $\omega(i) \notin\{j, j-1\}$. As in the proof of Lemma 6.7 define $a_{\omega}:=\varphi_{i, j}\left(\kappa_{\omega}\right)$. Observe that $\kappa_{\omega}(\tilde{\omega})=\kappa_{\omega}\left(\tilde{\omega}+e_{i}\right)=0$ for all $\tilde{\omega} \in \Omega$ with $\tilde{\omega}(i)=j-1$. Therefore, by Zero Contribution, we have $\varphi_{i, j}\left(\kappa_{\omega}\right)=0$, hence $a_{\omega}=0$.

Let. $h \in \tilde{H}$. Then, by Lemma 6.7 and the preceding argument,

$$
\begin{aligned}
\varphi_{i, j}(h) & =\sum_{\omega \in \Omega} a_{\omega} h(\omega) \\
& =\sum_{\omega \in \Omega:} a_{\omega(\hat{\omega} \in\{j, j-1\}} h(\omega) \\
& =\sum_{\omega \in \Omega:}\left[a_{\omega} h(\omega)+a_{\omega+e_{i}} h\left(\omega+e_{i}\right)\right] .
\end{aligned}
$$

We will show by induction that $a_{\omega+e_{\mathrm{a}}}=-a_{\omega}$ for all $\omega \in \Omega$ with $\omega(i)=j-1$. 
Suppose $\tilde{\omega} \in \Omega$ with $\tilde{\omega}(i)=j-1$, then $h_{\tilde{\omega}}\left(\omega+e_{i}\right)=h_{\bar{\omega}}(\omega)$ for every $\omega \in \Omega$ with $\omega(i)=j-1$. Therefore Zero Contribution implies

$$
\varphi_{i, j}\left(h_{\bar{\omega}}\right)=0 .
$$

Let now $\bar{\omega}$ be such that $\tilde{\omega}(i)=j-1$ and $\tilde{\omega}(k)=m$ for all $k \in N, k \neq i$. Then

$$
\begin{aligned}
\varphi_{i, j}\left(h_{\dot{\omega}}\right) & =\sum_{\omega \in \Omega:}\left[a_{\omega(i)=j-1} h_{\dot{\omega}}(\omega)+a_{\omega+e_{i}} h_{\bar{\omega}}\left(\omega+e_{i}\right)\right] \\
& =a_{\tilde{\omega}}+a_{\dot{\omega}+e_{i}} .
\end{aligned}
$$

Also, $\varphi_{i, j}\left(h_{\dot{\omega}}\right)=0$ by $(6.3)$, so that

$$
a_{\bar{\omega}+c_{i}}=-a_{\dot{j}}
$$

Next let $\tilde{\omega}$ be arbitrary with $\tilde{\omega}(i)=j-1$, and suppose as induction hypothesis that for all $\omega^{\prime} \geqslant \bar{\omega}$, with $\omega^{\prime} \neq \bar{\omega}$ and $\omega^{\prime}=j-1$ we have

$$
a_{\omega^{\prime}+r_{1}}=-a_{\omega^{\prime}} .
$$

Then

$$
\begin{aligned}
\varphi_{i, j}\left(h_{\bar{\omega}}\right) & =\sum_{\omega \in \Omega:}\left[a_{\omega(i)=j-1} h_{\dot{\omega}}(\omega)+a_{\omega+e_{i}} h_{\dot{\omega}}\left(\omega+e_{i}\right)\right] \\
& =\sum_{\omega \in \Omega: \omega(i)=j-1, \omega \geqslant \bar{\omega}}\left[a_{\omega}+a_{\omega+e_{i}}\right] \\
& =\sum_{\substack{\omega \in \Omega: \omega(i)=j-1, \omega \geqslant \bar{\omega}, \omega \neq \bar{\omega}\\
}}\left[a_{\omega}+a_{\omega+e_{i}}\right]+\left[a_{\bar{\omega}}+a_{\bar{\omega}+e_{i}}\right] \\
& a_{\bar{\omega}}+a_{\bar{\omega}+e_{i}},
\end{aligned}
$$

where the last equality follows by the induction hypothesis. Hence, with $\varphi_{i, j}\left(h_{\bar{\omega}}\right)=0$ by (6.3), we have

$$
a_{\bar{\omega}+e_{i}}=-a_{\dot{w}}
$$

Therefore, by induction,

$$
a_{\bar{\omega}+\varepsilon_{i}}=-a_{\bar{\omega}}
$$

for all $\tilde{\omega} \in \Omega$ with $\tilde{\omega}(i)=j-1$. Hence we can write

$$
\varphi_{i, j}(h)=\sum_{\omega \in \Omega: \omega(i)=j-1}\left[a_{\omega} h(\omega)-a_{\omega} h\left(\omega+e_{i}\right)\right] .
$$

The proof of the lemma is complete by taking $p_{\omega}:=-a_{\omega}$ and observing that these weights are independent of the situation $h \in \tilde{H}$. 
Lemma 6.14 Let $\varphi$ as in Lemma 6.13 additionally satisfy Distribution. Then the weights $p_{\omega}$ as in Lemma 6.13 sum to one.

Proof. Let $\bar{\omega}=(0,0, \ldots, 0)+j e_{i}$, then by Lemma 6.13

$$
\begin{aligned}
\varphi_{i, j}\left(h_{\dot{\omega}}\right) & =\sum_{\omega \in \Omega: \omega(i)=j-1} p_{\nu}\left[h_{\dot{\omega}}\left(\omega+e_{i}\right)-h_{\dot{\omega}}(\omega)\right] \\
& =\sum_{\omega \in \Omega:} p_{\omega(i)=j-1} .
\end{aligned}
$$

Distribution implies

$$
\sum_{k=1}^{n} \sum_{l=1}^{m} \varphi_{k, l}\left(h_{\tilde{\omega}}\right)=1
$$

and Zero Contribution implies $\varphi_{k, l}\left(h_{\dot{\omega}}\right)=0$ if $k \neq i$ and $\varphi_{i, l}\left(h_{\dot{\omega}}\right)=0$ if $l \neq \dot{\omega}(i)=j$. Hence

$$
1=\sum_{k=1}^{n} \sum_{l=1}^{m} \varphi_{k, l}\left(h_{\tilde{\omega}}\right)=\varphi_{i, j}\left(h_{\tilde{\omega}}\right)=\sum_{\omega \in \Omega:} p_{\omega(i)=j-1} .
$$

This completes the proof of the lemma.

Proof of Theorem 6.5. The Theorem follows from Lemmas 6.13 and 6.14 and the renaarks about the extension of homogeneous valuations to $\bar{H}$ preceding Lemma 6.7.

We proceed with proving Theorem 6.6. Like Theorem 6.5 we will prove Theorem 6.6 for the more general case of a linear map $\varphi: \vec{H} \rightarrow \mathbb{R}^{n \times(m+1)}$. More specifically, the theorem is implied by the following lemma, in which the condition of Attribute Symmetry is the obvious extension to this more general case.

In the proof of Lemma 6.15 we use the following definition. Call two attributes $i, k \in N$ symmetric in $h \in \tilde{H}$ if for the permutation $\sigma: N \rightarrow N$ with $\sigma(i)=k, \sigma(k)=i$, and $\sigma(l)=l$ for all $l \neq i, k$, we have $h(\omega)=\sigma h(\omega)$ for all $\omega \in \Omega$.

Lemma 6.15 Let $\varphi: \tilde{H} \rightarrow \mathbb{I R}^{n \times(m+1)}$ be linear and satisfy Attribute Symmetry and Zero Contribution. Let further $p_{\omega}$ be the weights determined in Lemma 6.13. Then, for every permutation $\sigma$ of $N$.

$$
p_{\omega}^{i}=p_{\sigma \omega}^{\sigma(i)} .
$$


Proof. Lemma 6.13 implies that for every $i \in N$ and $j \in M, j \neq 0$ there is a collection of real numbers $\left\{p_{\omega}: \omega \in \Omega\right.$ with $\left.\omega(i)=j-1\right\}$ with

$$
\varphi_{i, j}(h)=\sum_{\omega \in \Omega:} p_{\omega(i)=j-1}\left[h\left(\omega+e_{i}\right)-h(\omega)\right]
$$

for all $h \in \tilde{H}$.

Suppose that attributes $1,2 \in N$ are symmetric in $h \in \bar{H}$. Hence, for the permutation $\sigma: N \rightarrow N$, with $\sigma(1)=2, \sigma(2)=1$ and $\sigma(i)=i$ if $i \neq 1,2$, we have $h(\omega)=\sigma h(\omega)$ for all $\omega \in \Omega$. Then Attribute Symmetry implies

$$
\varphi_{1, j}(h)=\varphi_{2, j}(h),
$$

for all $j=1, \ldots, m$. Substituting the expression above we obtain

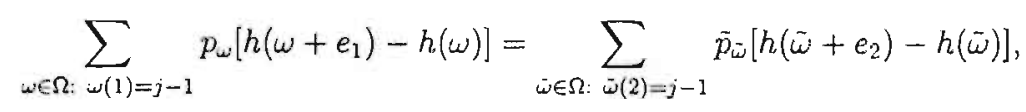

where $p_{\omega}=p_{\omega}^{1}$ and $\bar{p}_{\bar{\omega}}=p_{\bar{\omega}}^{2}$. Because $\sigma h=h$ this implies

$$
0=\sum_{\omega \in \Omega: \omega(1)=j-1} p_{\omega}\left[h\left(\omega+e_{1}\right)-h(\omega)\right]-\sum_{\nu \in \Omega: \dot{\omega}(2)=j-1} \tilde{p}_{\tilde{\omega}}\left[\sigma h\left(\tilde{\omega}+e_{2}\right)-\sigma h(\tilde{\omega})\right],
$$

and using $\sigma h\left(\sigma \omega^{\prime}\right)=h\left(\omega^{\prime}\right)$ we obtain

$$
\begin{aligned}
& 0=\sum_{\omega \in \Omega:} p_{\omega(1)=j-1}\left[h\left(\omega+e_{1}\right)-h(\omega)\right]-\sum_{\tilde{\omega} \in \Omega:} \tilde{p}_{\tilde{\omega}(2)=j-1}\left[h\left(\sigma^{-1}\left(\tilde{\omega}+e_{2}\right)\right)-h\left(\sigma^{-1} \tilde{\omega}\right)\right] \\
& =\sum_{\omega \in \Omega: \omega(1)=j-1} p_{\omega}\left[h\left(\omega+e_{1}\right)-h(\omega)\right]-\sum_{\omega \in \Omega: \omega(1)=j-1} \tilde{p}_{\omega}\left[h\left(\sigma^{-1} \tilde{\omega}+e_{1}\right)-h\left(\sigma^{-1} \tilde{\omega}\right)\right] \\
& =\sum_{\omega \in \Omega: \omega(1)=j-1} p_{\omega}\left[h\left(\omega+e_{1}\right)-h(\omega)\right]-\sum_{\omega \in \Omega: \omega(1)=j-1} \tilde{p}_{\sigma \omega}\left[h\left(\omega+e_{1}\right)-h(\omega)\right]
\end{aligned}
$$

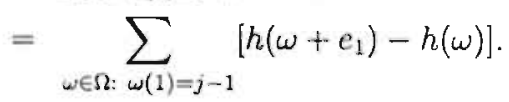

Let $\omega^{\prime} \in \Omega$ such that $\omega^{\prime}=\left(j-1, l, \omega^{\prime}(3), \ldots, \omega^{\prime}(n)\right)$ for some $l \in M \backslash\{0\}$. We define the situation $h$ by

$$
h:=\kappa_{\omega^{\prime}}+\kappa_{\sigma \omega^{\prime}} .
$$

Then the attributes 1 and 2 are symmetric in $h$. For $\omega \in \Omega$ with $\omega(1)=j-1$ we have

$$
h(\omega)= \begin{cases}1, & \text { if } \omega=\omega^{\prime} \text { and } l \neq j-1 \\ 2, & \text { if } \omega=\omega^{\prime} \text { and } l=j-1 \\ 0, & \text { otherwise. }\end{cases}
$$


Therefore

$$
\begin{aligned}
0 & =\sum_{\omega \in \Omega:}\left[h\left(\omega+e_{1}\right)-h(\omega)\right] \\
& = \begin{cases}2\left[p_{\omega^{\prime}}-\hat{p}_{\sigma \omega^{\prime}}\right], & \text { if } l=j-1 \\
p_{\omega^{\prime}}-\bar{p}_{\sigma \omega^{\prime}}, & \text { otherwise, }\end{cases}
\end{aligned}
$$

or equivalently $p_{\omega^{\prime}}=\bar{p}_{\sigma \omega^{\prime}}$. Because the choice of $l, j \in M \backslash\{0\}$ was arbitrary we conclude that $p_{\omega}=\tilde{p}_{\sigma \omega}$ for any $\omega \in \Omega$.

The proof for any pair of symmetric attributes $i, k \in N$ is similar, so that the theorem follows from the fact that every permutation can be written as a sequence of 2-attribute exchanges.

We proceed with the proof of Theorem 6.7, the characterization of the egalitarian evaluation.

Proof of TheOREM 6.7. The proof of the if-part of the theorern is left to the reader.

For the only-if part, let $\varphi$ be a homogeneous valuation satisfying Zero Contribution, Distribution, Attribute Symmetry and Level Symmetry. By Theorem 6.1 it is sufficient to prove that $\varphi$ coincides with the egalitarian valuation on all simple situations.

Let $k \in\{0, \ldots, n-1\}$ be arbitrary, and let $O \subset N$ be any collection of $k$ distinct. attributes. Consider $\omega \in \Omega$ with $\omega(i)=0$ for all $i \in O$ and $\omega(i)>0$ for all $i \in N \backslash O$. Distribution implies

$$
1=\sum_{i=1}^{n} \sum_{j=0}^{m} \varphi_{i, j}\left(h_{\omega}\right),
$$

and Zero Contribution implies $\varphi_{i, j}\left(h_{\omega}\right)=0$ for all $i \in O$ and $\varphi_{i, j}\left(h_{\omega}\right)=0$ for all $i \in N \backslash O$ if $j \neq \omega(i)$. Substitution into the above equation gives

$$
1=\sum_{i \in N \backslash O} \varphi_{i, \omega(i)}\left(h_{\omega}\right)
$$

We show by induction, that $\varphi_{i, \omega(i)}\left(h_{\omega}\right)=1 /(n-k)$ for all $i \in N \backslash O$. Let $l:=\max _{i \in N \backslash Q}\{\omega(i)\}$. If for all $i \in N \backslash O$ we have $\omega(i)=l$, then we conclude by Attribute Symmetry that $\varphi_{i, \omega(i)}\left(h_{\omega}\right)=1 /(n-k)$ for all these $i$.

Let there exist an $s \in N \backslash O$ such that $\omega(s)<l$. Assume that for ail $\bar{\omega} \in \Omega$ with $\tilde{\omega}(i)=0$ for all $i \in O$ and $\omega(i)>0$ for all $i \in N \backslash O$, such that

$$
|\{i \in N \backslash O: \bar{\omega}(i)=l\}|>|\{i \in N \backslash O: \omega(i)=l\}|
$$


we have $\varphi_{i, \omega(2)}\left(h_{\dot{\omega}}\right)=1 /(n-k)$.

Take any $s \in N \backslash O$ such that $\omega(s)<l$. Define $\omega^{\prime}$ as follows

$$
\omega^{\prime}(i)= \begin{cases}l, & \text { if } i=s \\ \omega(i), & \text { otherwise, }\end{cases}
$$

and define the situation $h:=h_{\omega}+h_{\omega^{\prime}}$. Then, it is easy to show that the levels $\omega(s)$ and $l$ are symmetric for $s$ in $h$. Hence, by Level Symmetry we have $\varphi_{s, \omega}(s)(h)=\varphi_{s, l}(h)$. With the Transfer Property this implies that

$$
\varphi_{s, \omega(s)}\left(h_{\omega}\right)+\varphi_{s, \omega(s)}\left(h_{\omega^{\prime}}\right)=\varphi_{s, l}\left(h_{\omega}\right)+\varphi_{s, l}\left(h_{\omega^{\prime}}\right) .
$$

Note also that by Zero Contribution (and the fact that $\omega(s) \neq l=\omega^{\prime}(s)$ ) we have $\varphi_{s, \omega(s)}\left(h_{\omega^{\prime}}\right)=\varphi_{s, l}\left(h_{\omega}\right)=0$ so that

$$
\varphi_{s, \omega(s)}\left(h_{\omega}\right)=\varphi_{s, l}\left(h_{\omega^{\prime}}\right)
$$

By the induction assumption $\varphi_{s, l}\left(h_{\omega^{\prime}}\right)=1 /(n-k)$ for all $i \in N \backslash O$. Therefore $\varphi_{s, \omega(s)}\left(h_{\omega}\right)=$ $1 /(n-k)$. By induction it follows that $\varphi_{i, \omega(i)}\left(h_{\omega}\right)=1 /(n-k)$ for all $i \in N \backslash O$ such that $\omega(i)<l$.

Recall now that by Distribution and Zero Contribution

$$
1=\sum_{i \in N \backslash O} \varphi_{i, \omega(i)}\left(h_{\omega}\right)
$$

which by the above findings is equivalent to

$$
\begin{aligned}
1 & =\sum_{i: 0<\omega(i)<l} \varphi_{i, \omega(i)}\left(h_{\omega}\right)+\sum_{i: \omega(i)=l} \varphi_{i, \omega(i)}\left(h_{\omega}\right) \\
& =\sum_{i: 0<\omega(i)<l} \frac{1}{n-k}+\sum_{i: \omega(i)=l} \varphi_{i, \omega(i)}\left(h_{\omega}\right) .
\end{aligned}
$$

Using Attribute Symmetry in the sercond sum we find that $\varphi_{i, \omega(i)}\left(h_{\omega}\right)=1 /(n-k)$ for all $i \in N \backslash O$. Hence, $\varphi=\varepsilon$ for this case.

Because $O$ was an arbitrary selection of $k$ distinct attributes, and $k \in\{0, \ldots, n-1\}$ was arbitrary too, we have $\varphi=\varepsilon$ on the set of simple situations $S$. This concludes the proof of the theorem.

We conclude this Appendix with the proof of Theorem 6.8 describing the values assigned by the egalitarian valuation to the situations $\kappa_{\omega} \in \tilde{H}$. 
Proof of Theorem 6.8. In this proof we identify the egalitarian valuation with its linear extension on $\tilde{H}$, the set of situations which do not necessarily satisfy the monotonicity requirement. First we prove Statement (iv) of Theorem 6.8. For $\omega \in \Omega \backslash\{(0, \ldots, 0)\}$ define the situation $\hat{h}_{\omega}:=h_{\omega}-\kappa_{\omega}$. Let $l_{1}, l_{2} \in\{1, \ldots, m-1\}$ and $\bar{\omega}:=\left(l_{1}, l_{2}, m, \ldots, m\right)$. Then,

$$
\ddot{h}_{\tilde{\omega}}:=h_{\dot{\omega}+e_{1}}+h_{\dot{\omega}+c_{2}}-h_{\dot{\omega}+e_{1}+e_{2}} \text {. }
$$

Therefore, by the definition of the egalitarian valuation and linearity, we conclude $\varepsilon\left(\hat{h}_{\bar{\omega}}\right)=$ $\varepsilon\left(h_{\dot{\omega}}\right)$. Hence, by linearity of $\varepsilon$ and $\hat{h}_{\dot{\omega}}=h_{\bar{\omega}}-\kappa_{\dot{\omega}}$, we find $\varepsilon\left(\kappa_{\dot{\omega})}\right)=0_{n \times(m+2)}$ (here $0_{n \times(m+1)}$ denotes the $n \times(m+1)$ matrix with all entries zero).

Assume now that for $\tilde{\omega}=\left(l_{1}, l_{2}, \tilde{\omega}(3), \ldots, \tilde{\omega}(n)\right)$ with $l_{1}, l_{2} \in\{1, \ldots, m-1\}$ we have

$$
\varepsilon\left(\kappa_{\omega}\right)=0_{n \times(m+1)} \text { if } \omega \geqslant \tilde{\omega} \text { but } \omega \neq \tilde{\omega} \text {. }
$$

We show $\varepsilon\left(\kappa_{\dot{\omega}}\right)=0_{n \times(m+1)}$. We decompose $\hat{h}_{\bar{\omega}}$ as follows

$$
\hat{h}_{\dot{\omega}}:=h_{\bar{\omega}+e_{1}}+h_{\bar{\omega}+e_{2}}-h_{\dot{\omega}+e_{1}+e_{2}}-\sum_{\substack{\omega \geqslant \bar{\omega}, \omega \neq \dot{\omega} \\ \omega \neq \bar{\omega}+e_{1} \\ \omega \neq \bar{\omega}+e_{2}}} \kappa_{\dot{\omega}} .
$$

Then, by linearity of $\varepsilon$, the definition of $\varepsilon$ for simple situations, and the assumption above we find $\varepsilon\left(\hat{h}_{\dot{\omega}}\right)=\varepsilon\left(h_{\dot{\omega}}\right)$. Therefore, by linearity of $\varepsilon$ and $\hat{h}_{\dot{\omega}}=h_{\dot{\omega}}-\kappa_{\dot{\omega}}$, we find $\varepsilon\left(\kappa_{\dot{\omega}}\right)=0_{n \times(m+1)}$. Hence, by induction Statement (iv) of the theorem follows.

In order to prove Statement (i) assume $k=0$ and $j=m$. Then $\omega_{k, j}=\omega_{0, m}=$ $(m, \ldots, m)$. Hence $\kappa_{\omega_{k, j}}=\kappa_{\omega_{0, m}}=h_{(m, \ldots, m)}$ and, by Definition 6.4 of the egalitarian valuation, the proof of the statement follows.

Now we prove Statement (iii) for $k=0$ : Let $j \in\{1, \ldots, m-1\}$. Recall that $\omega_{0, j}=$ $(j, m, \ldots, m)$. We use the representation $\kappa_{\omega_{0, j}}=h_{\omega^{*}, j}-h_{\omega_{0, j+1}}$, additivity of $\varepsilon$, and the definition of $\varepsilon\left(h_{\omega_{0, j}}\right)$ and $\varepsilon\left(h_{\omega_{0, j+1}}\right)$ to conclude:

$$
\varepsilon_{s, e}\left(\kappa_{\omega_{0,2}}\right)=\frac{1}{n} \cdot\left\{\begin{array}{cc}
1, & \text { for } s=1 \text { and } t=j, \\
-1, & \text { for } s=1 \text { and } t=j+1 \\
0, & \text { elsewhere. }
\end{array}\right.
$$

Next we prove Statements (ii) and (iii) by induction. Let $k=1$. To prove Statement (ii) note that $\omega_{1, m}=(0, m, \ldots, m)$ and $\kappa_{\omega_{1, m}}=h_{\omega_{1, m}}-h_{\omega_{0,1}}$, with $\omega_{0,1}=(1, m, \ldots, m)$. 
Hence, by additivity of $\varepsilon$ and the definition of $\varepsilon\left(h_{\omega_{1, m}}\right)$ and $\varepsilon\left(h_{\omega_{0,1}}\right)$, we find

$$
\begin{aligned}
& \varepsilon\left(\kappa_{\omega_{1, m}}\right)=\frac{1}{n-1} \cdot\left(\begin{array}{cccc}
0 & 0 & \cdots & 0 \\
0 & 0 & \cdots & 0 \\
\vdots & \vdots & \ddots & \vdots \\
0 & 0 & \cdots & 0 \\
0 & 1 & \cdots & 1
\end{array}\right)-\frac{1}{n} \cdot\left(\begin{array}{cccc}
1 & 0 & \cdots & 0 \\
0 & 0 & \cdots & 0 \\
\vdots & \vdots & \ddots & \vdots \\
0 & 0 & \cdots & 0 \\
0 & 1 & \cdots & 1
\end{array}\right) \\
& =\frac{1}{n \cdot(n-1)} \cdot\left(\begin{array}{ccc}
1-n & 0 \cdots 0 \\
0 & 0 \cdots 0 \\
\vdots & \vdots & \ddots \\
0 & 0 \cdots 0 \\
0 & 1 \cdots 1
\end{array}\right)
\end{aligned}
$$

which implies Statement (ii) for $k=1$.

To prove Statement (iii) for $k=1$, note that for $j \in\{1, \ldots, m-1\}$ we focus on $\kappa_{(0, j, m, \ldots, m)}$. We can decompose $\kappa_{(0, j, m, \ldots, m)}$ as follows

$$
\kappa_{(0, j, m, \ldots, m)}=h_{(0, j, m, \ldots, m)}-h_{(0, j+1, m, \ldots, m)}-\sum_{l=1}^{m} \kappa_{(i, j+1, m, \ldots, m)} .
$$

Using the results for Statement (iv) derived above, and additivity of $\varepsilon$ we get

$$
\varepsilon\left(\kappa_{(0, j, m, \ldots, m)}\right)=\varepsilon\left(h_{(0, j, m, \ldots, m)}\right)-\varepsilon\left(h_{(0, j+1, m, \ldots, m)}\right)-\varepsilon\left(\kappa_{(m, j, m, \ldots, m)}\right)
$$

Attribute Symmetry and the results for Statement (iii) if $k=0$ determine the value $\varepsilon\left(\kappa_{(m, j, m, \ldots, m)}\right)$. Moreover, by Definition 6.4, we know $\varepsilon\left(h_{(0, j, m, \ldots, m)}\right)$ and $\varepsilon\left(h_{(0, j+1, m, \ldots, m)}\right)$. Substitution in the formula above then gives

$$
\varepsilon_{s, \ell}\left(\kappa_{\omega_{1, j}}\right)=\frac{1}{n(n-1)} \cdot\left\{\begin{array}{cc}
1, & \text { for } s=2 \text { and } t=j \\
-1, & \text { for } s=2 \text { and } t=j+1 \\
0, & \text { elsewhere. }
\end{array}\right.
$$

Hence we proved Statement (iv), Statement (i), and the Statements (ii) and (iii) for $k \leqslant 1$. Let now $k \in\{2, \ldots, n-1\}$, and assume that the Statements (ii) and (iii) hold for all $k^{\prime}<k$. We first show that Statement (ii) holds for $k$. 
Note that $\omega_{k, m}=(\underbrace{0, \ldots, 0}_{k}, m, \ldots, m)$. It is easy to see that Zero Contribution and Attribute Symmetry imply

$$
\varepsilon_{s, l}\left(\kappa_{\cup_{k, m}}\right)=\left\{\begin{array}{cc}
a, & \text { for } s=1, \ldots, k \text { if } t=1 \\
b, & \text { for } s=k+1, \ldots, n \text { if } t=m \\
0, & \text { otherwise }
\end{array}\right.
$$

for some $a, b \in \mathbb{R}$. Moreover, Distribution implies $k \cdot a+(n-k) \cdot b=0$. It is therefore sufficient to determine $a$ (or $b$ ).

The following decomposition holds for $\kappa_{\omega_{k, m}}$ :

$$
\kappa_{\omega_{k, m}}=h_{\omega_{k, m}}-h_{\omega_{k-1,1}}-\sum_{i_{1}=0}^{m} \cdots \sum_{i_{k-1}=0}^{m} \kappa_{\left(i_{1}, \ldots, i_{k-1}, 0_{1}, m, \ldots, m\right)},
$$

with $\sum_{i=1}^{k-1} i_{l}>0$ (so that $\left(i_{1}, \ldots, i_{k-1}, 0, m, \ldots, m\right) \neq w_{k, m}$ ). The states in the above sum can be separated into collections of states according to the Statements (ii)-(iv).

For $L \subseteq\{1, \ldots, k-1\}$ we define the state $\omega_{k, L}$ by

$$
\omega_{k, L}:=\left(t_{1}, \ldots, t_{k-1}, 0, m, \ldots, m\right)
$$

with $t_{r}=0$ for all $r \in L$ and $t_{r}=m$ elsewhere, and for $l=0, \ldots, k-2$ we define the collection of states

$$
\Omega_{k, l}:=\bigcup_{|L|=l}\left\{\omega_{k, L}\right\}
$$

Note that for each $l=1, \ldots, k-2$, if $\omega \in \Omega_{k, t}$ we know $\varepsilon_{s, t}\left(\kappa_{\omega}\right)$ by Statement (ii) and the induction assumption.

For $u=1, \ldots, k-1, t_{u}=1, \ldots, m-1$ and $L(u) \subseteq\{1, \ldots, k-1\} \backslash\{u\}$ we define the states $\omega_{k, L,(u)}$ by

$$
\omega_{k, L(u)}:=\left(t_{1}, \ldots, t_{k-1}, 0, m, \ldots, m\right)
$$

with $t_{v}=0$ for all $v \in L(u)$ and $t_{v}=m$ elsewhere if $v \neq u$, and for $l=0, \ldots, k-2$ we define the collection of states

$$
\Omega_{k, l, u}:=\bigcup_{t_{u}=1|L(u)|=t}^{m-1}\left\{\omega_{k, L(u)}\right\} .
$$

Note that for any $u=1, \ldots, k-1$ and any $l=1, \ldots, k-2$, if $\omega \in \Omega_{k, l, u}$ we know $\varepsilon_{s, t}\left(\kappa_{\omega}\right)$ by Statement (iii) and the induction assumption. Further if $\omega=\left(i_{1}, \ldots, i_{k-1}, 0, m_{1} \ldots, m\right)$ 
and $\omega \notin \Omega_{k, l, u} \cup \Omega_{k, l}$, then we know $\varepsilon_{s, t}\left(\kappa_{\omega}\right)$ by Statement (iv). Hence, applying $\varepsilon$ to equation (6.5), linearity of $\varepsilon$, and Statement (iv) we find

$$
\varepsilon\left(\kappa_{\omega_{k, m}}\right)=\varepsilon\left(h_{\omega_{k, m}}\right)-\varepsilon\left(h_{\omega_{k-1,1}}\right)-\sum_{u=1}^{k-1} \sum_{l=0}^{k-2} \sum_{\omega \in \Omega_{k, l, l}}^{m-1} \varepsilon\left(\kappa_{\omega}\right)-\sum_{l=0}^{k-2} \sum_{\omega \in \Omega_{k, l}} \varepsilon\left(\kappa_{\omega}\right) .
$$

Recall that according to equation (6.4) we have $a=\varepsilon_{1,1}\left(\kappa_{w_{k, m}}\right)$. By Definition 6.4 we have $\varepsilon_{1,1}\left(h_{\omega_{k, m}}\right)=\varepsilon_{1,1}\left(h_{\omega_{k-1,1}}\right)=0$. For a fixed $u \in\{1, \ldots, k-1\}$, by the induction assumption and Attribute Symmetry, we find for the value of $\varepsilon\left(\kappa_{\omega}\right)$ with $\omega \in \Omega_{k, l, u}$ :

$$
\varepsilon_{s, t}\left(\kappa_{\omega}\right)=\frac{(l+1) !}{\prod_{r=0}^{l+1}(n-r)} \cdot\left\{\begin{array}{cc}
1, & \text { for } s=u \text { and } t=\omega(u) \\
-1, & \text { for } s=u \text { and } t=\omega(u)+1 \\
0, & \text { elsewhere, }
\end{array}\right.
$$

so that with the cardinality $\left|\Omega_{k, l, u}\right|=\left(\begin{array}{c}k-2 \\ l\end{array}\right)$ we find

$$
\sum_{\substack{\omega \in \Omega_{k, l, u} \\
\omega(u)=1}}^{m-1} \varepsilon_{s, l}\left(\kappa_{\omega}\right)=\left(\begin{array}{c}
k-2 \\
l
\end{array}\right) \cdot \frac{(l+1) !}{\prod_{r=0}^{l+1}(n-r)} \cdot\left\{\begin{array}{cc}
1, & \text { for } s=u \text { and } t=1 \\
-1, & \text { for } s=u \text { and } t=m \\
0, & \text { elsewhere, }
\end{array}\right.
$$

and

$$
\sum_{\substack{l=0 \\
\omega \in \Omega_{k, l, u} \\
\omega(u)=1}}^{k-2} \sum_{s, t}\left(\kappa_{\omega}\right)=\alpha \cdot\left\{\begin{array}{cc}
1, & \text { for } s=u \text { and } t=1 \\
-1, & \text { for } s=u \text { and } t=m \\
0, & \text { elsewhere }
\end{array}\right.
$$

with

$$
\alpha:=\sum_{l=0}^{k-2}\left(\begin{array}{c}
k-2 \\
l
\end{array}\right) \cdot \frac{(l+1) !}{\prod_{r=0}^{l+1}(n-r)} .
$$

Therefore we conclude that for $s=1$ and $t=1$ we have

$$
\sum_{u=1}^{k-1} \sum_{l=0}^{k-2} \sum_{\substack{\omega \in \Omega_{k, l, u} \\ \omega(u)=1}}^{m-1} \varepsilon_{1,1}\left(\kappa_{\omega}\right)=\alpha
$$

Again we use the induction assumption and Attribute Symmetry to compute $\varepsilon_{1_{1} 1}\left(\kappa_{\omega}\right)$ for $\omega \in \Omega_{k, l}:$

$$
\varepsilon_{s, t}\left(\kappa_{\omega}\right)=\frac{l !}{\prod_{r=0}^{l+1}(n-r)} \cdot\left\{\begin{array}{cc}
l+1-n, & \text { for } t=1 \text { if } \omega(s)=0 \\
l+1, & \text { for } t=m \text { if } \omega(s)=m \\
0, & \text { elsewhere. }
\end{array}\right.
$$


Therefore if $l=0$ we have

$$
\sum_{\omega \in \Omega_{k, 0}} \varepsilon_{s, t}\left(\kappa_{\omega}\right)=\frac{1}{n(n-1)} \cdot\left\{\begin{array}{cc}
1-n, & \text { for } s=k \text { and } i=1 \\
1, & \text { for } s \neq k \text { and } i=m \\
0, & \text { elsewhere, }
\end{array}\right.
$$

implying $\varepsilon_{1,1}\left(\kappa_{\omega}\right)=0$ for all $\omega \in \Omega_{k, 0}$. And if $0<l<k-1$ we have

$$
\sum_{\omega \in \Omega_{k, t}} \varepsilon_{1,1}\left(\kappa_{\omega}\right)=\frac{l !}{\prod_{r=0}^{l+1}(n-r)} \cdot\left(\left(\begin{array}{c}
k-1 \\
l
\end{array}\right)-\left(\begin{array}{c}
k-2 \\
l
\end{array}\right)\right] \cdot(l+1-n) .
$$

Here $\left(\begin{array}{c}k-1 \\ i\end{array}\right)=\left|\Omega_{k, l}\right|$ and $\left(\begin{array}{c}k-2 \\ l\end{array}\right)$ equals the number of states $\omega \in \Omega_{k, l}$ for which $\varepsilon_{1,1}\left(\kappa_{w}\right)=0$.

Hence, we find

$$
\begin{aligned}
& \sum_{l=0}^{k-2} \sum_{\omega \in \mathbb{R}_{k, l}} \varepsilon_{1,1}\left(\kappa_{\omega}\right)=\sum_{l=0}^{k-2} \frac{l !}{\prod_{r=0}^{++1}(n-r)} \cdot\left[\left(\begin{array}{c}
k-1 \\
l
\end{array}\right)-\left(\begin{array}{c}
k-2 \\
l
\end{array}\right)\right] \cdot(l+1-n) \\
&=\sum_{l=1}^{k-2}\left(\begin{array}{c}
k-2 \\
l-1
\end{array}\right) \cdot(l+1-n) \cdot \frac{l !}{\prod_{r=0}^{l+1}(n-r)} \\
&=-\sum_{l=1}^{k-2}\left(\begin{array}{c}
k-2 \\
l-1
\end{array}\right) \cdot \frac{l !}{\prod_{r=0}^{l}(n-r)} \\
&=-\sum_{l=0}^{k-3}\left(\begin{array}{c}
k-2 \\
l
\end{array}\right) \cdot \frac{(l+1) !}{l+1} \\
&=-a+\frac{(k-1) !}{\prod_{r=0}^{k-1}(n-r)} \\
& \prod_{r=0}^{k}(n-r)
\end{aligned}
$$

Summarizing, we find

$$
a=0+0-\alpha+\alpha-\frac{(k-1) !}{\prod_{r=0}^{k-1}(n-r)} .
$$

Substitution into $k \cdot a+(n-k) \cdot b=0$, gives

$$
b=\frac{k}{n-k} \cdot \frac{(k-1) !}{\prod_{r=0}^{k-1}(n-r)} .
$$


Therefore we conclude Statement (ii) for $k$.

Next we prove Statement (iii) for $k$. Let $j \in\{1, \ldots, m-1\}$ be arbitrarily chosen. Note that $\omega_{k, j}=(\underbrace{0, \ldots, 0}_{k}, j, m, \ldots, m)$. We can write $\kappa_{\omega_{k, j}}$ in the following way:

$$
\kappa_{\omega_{k, j}}=h_{\omega_{k, j}}-h_{\omega_{k, j+1}}-\sum_{i_{1}=0}^{m} \cdots \sum_{i_{k}=0}^{m} \kappa_{\left(i_{1}, \ldots, i_{k}, j, m, \ldots, m\right)},
$$

with $\sum_{v=1}^{k} i_{v}>0$ (such that $\left.\left(i_{1}, \ldots, i_{k}, j, m, \ldots, m\right) \neq \omega_{k, j}\right)$.

For $u=k+1$ and $L(k+1) \subseteq\{1, \ldots, k\}$ recall the definition the state $\omega_{k, L(k+1)}$ by

$$
\omega_{k, L(k+1)}:=\left(t_{1}, \ldots, t_{k}, \omega(k+1), m, \ldots, m\right)
$$

where $t_{v}=0$ for all $v \in L(k+1)$ and $t_{v}=m$ elsewhere. Now, with $\omega(k+1)=j$ fixed, for $l=0, \ldots, k-1$ we define the collection of states

$$
\hat{\Omega}_{k, l}:=\bigcup_{|L(k+1)|=l}\left\{\omega_{k, L(k+1)}: \omega(k+1)=j\right\} .
$$

Now we apply $\varepsilon$ to $\kappa_{\omega_{k, j}}$ in equation (6.6), and conclude by linearity and Statement (iv), that

$$
\varepsilon\left(\kappa_{\omega_{k, j}}\right)=\varepsilon\left(h_{\omega_{k, j}}\right)-\varepsilon\left(h_{\omega_{k, j+1}}\right)-\sum_{l=0}^{k-1} \sum_{\omega \in \hat{\Omega}_{k, l}} \varepsilon\left(\kappa_{\omega}\right) .
$$

Substitution of $\varepsilon\left(h_{\omega_{k, j}}\right), \varepsilon\left(h_{\omega_{k, j+1}}\right)$ (which are known by Definition 6.4) and of $\varepsilon\left(\kappa_{\omega}\right)$ for $\omega \in \hat{\Omega}_{k, l}$ (which are known by the induction assumption and Attribute Symmetry) implies

$$
\varepsilon_{t, s}\left(\kappa_{\omega}\right)=\left[\frac{1}{n-k}-\sum_{l=0}^{k-1}\left(\begin{array}{l}
k \\
l
\end{array}\right) \cdot \frac{l !}{\prod_{r=0}^{l}(n-r)}\right] \cdot\left\{\begin{array}{cc}
1, & \text { for } s=k+1 \text { and } t=j \\
-1, & \text { for } s=k+1 \text { and } t=j+1 \\
0, & \text { elsewhere }
\end{array}\right.
$$

where $\left(\begin{array}{l}k \\ l\end{array}\right)=\left|\hat{\Omega}_{k, l}\right|$. Define

$$
c:=\frac{1}{n-k}-\sum_{l=0}^{k-1}\left(\begin{array}{l}
k \\
l
\end{array}\right) \cdot \frac{l !}{\prod_{r=0}^{l}(n-r)} .
$$

Then we find

$$
c=\frac{1}{n-k}-\sum_{l=1}^{k} \frac{k !}{(k-l+1) !} \cdot \frac{1}{\prod_{r=0}^{l-1}(n-r)}
$$




$$
\begin{aligned}
& =\frac{1}{\prod_{r=0}^{k}(n-r)} \cdot\left\{\prod_{r=0}^{k-1}(n-r)-\sum_{l=1}^{k}\left[\frac{k !}{(k-l+1) !} \cdot \prod_{r=l}^{k}(n-r)\right]\right\} \\
& =\frac{k !}{\prod_{r=0}^{k}(n-r)} .
\end{aligned}
$$

Hence we get Statement (iii) of the theorem for $k$.

Summarizing, we conclude the validity of the Statements (ii) and (iii) for $k$, and by induction for all $0 \leqslant k<n$. This concludes the proof of Theorem 6.8 . 



\section{References}

Abdellaoui, M. (1998), "Eliciting Utilities and Decision Weights under Cumulative Prospect Theory," working paper, GRID, ENS, Cachan, France.

Anscombe, F.J. \& R.J. Aumann (1963), "A Defnition of Subjective Probability," Annals of Mathematical Statistics 34, 199-205.

Arrow, K.J. (1964), "The Role of Securities in the Optimal Allocation of Risk-Bearing," Review of Economic Studies 31, 91-96.

Atkinson, A.B. (1970), "On the Measurement of Inequality," Joumal of Economic Theory $2,244-263$.

Barten, A.P. \& V. Böhm (1982), "Consumer Theory." In K.J. Arrow \& M.D. Intriligator (Eds.), Handbook of Mathematical Economics II, North-Holland, Amsterdam, Chapter $9,381-429$.

Bateman, I., A. Munro, B. Rhodes, C. Starmer, \& R. Sugden (1997), "A Test of the Theory of Reference-Dependent Preferences," The Quarterly Journal of Economics $112,647 \cdot 601$.

Beetsma, R.M.W.J. \& P.C. Schotman (1997), "Measuring Risk Attitudes in a Natural Experiment: An Empirical Analysis of the Television Game Show LINGO," Maastricht University, The Netherlands.

Bell, D.E. (1982), "Regret in Decision Making under Uncertainty," Operations Research $30,961-981$.

Bell, D.E. (1988), "One-Switch Utility Functions and a Measure of Risk." Management Sciences 34, 1416-1424.

Ben-Porath, E. \& I. Gilboa (1994), "Linear Measures, the Gini Index, and the IncomeEquality Tradeoff," Journal of Economic Theory 64, 443-467.

Ben-Porath, E., I. Gilboa, \& D. Schmeidler (1997), "On the Measurement of Inequality under Uncertainty," Joumal of Economic Theory 75, 194-204.

Benartzi, S. \& R.H. Thaler (1995), "Myopic Loss Aversion and the Equity Premium Puz- 
ale," The Quarterly Journal of Economics 110, 73-92.

Bernstein, L.M., G. Chapman, C. Christensen, D. Potts, \& A.S. Elstein (1997), "Five Models of Choice between Multioutcome Lotteries," Journal of Behavioral Decision Making 10, 93-115.

Binswanger, H. (1981), "Attitudes towards Risk: Theoretical Implications of an Experiment in Rural India," Economic Journal 91, 867-890.

Blackorby, C., R. Davidson, \& D. Donaldson (1977), "A Homiletic Exposition of the Expected Utility Hypothesis," Economica 44, 351-358.

Bleichrodt, H. \& J. Pinto (1998), "The Validity of QALYs when Expected Utility Theory no Longer Holds," iMTA, Erasmus University, Rotterdam, The Netherlands.

Bleichrodt, H. \& J. Quiggin (1997), "Characterizing QALYs under a General Rank Dependent Utility Model," Journal of Risk and Uncertainty, 15, 151-165.

Bouzit, A.M. \& G. Gleyses (1996), "Empirical Estimation of RDEU Preference Functional in Agricultural Production," GRID, ENS, Cachan, France.

Broome, J. (1991), "Weighing Goods." Basil Blackwell, Oxford.

Camerer, C.F. \& T.-H. Ho (1994), "Violations of the Betweenness Axiom and Nonlinearity in Probability," Joumal of Risk and Uncertainty 8, 167-196.

Cass, D. \& J.E. Stiglitz (1972), "Risk Aversion and Wealth Effects on Portfolios with Many Assets," Review of Economic Studies 39, 331-354.

Chateauneuf, A. (1991), "On the Use of Capacities in Modeling Uncertainty Aversion and Risk Aversion," Joumal of Mathematical Economics 20, 343-369.

Chateauneuf, A. (1996a), "Comonotonicity Axioms and Rank-Dependent Expected Utility Theory for Arbitrary Consequences," CERMSEM, University of Paris I.

Chateauneuf, A. (1996b), "Decreasing Inequality; An Approach through Non-Additive Models," CERMSEM 96.58, University of Paris I.

Chateauneuf, A. \& P.P. Wakker (1993), "From Local to Global Additive Representation," Journal of Mathematical Economics 22, 523-545.

Chechile, R.A. \& A.D.J. Cooke (1997), "An Experimental Test of a General Class of Utility Models: Evidence for Context Dependence," Journal of Risk and Uncertainty $14,75-93$.

Chew, S.H. (1989), "The Rank-Dependent Quasilinear Mean," Unpublished manuscript, Department of Economics, University of California, Irvine, USA.

Chew; S.H. \& L.G. Epstein (1989), "A Unifying Approach to Axiomatic Non-Expected Utility Theories," Journal of Economic Theory 49, 207-240. 
Chew, S.H. \& E. Karni (1994), "Choquet Expected Utility with a Finite State Space: Commutativity and Act-Independence," Joumal of Economic Theory 62, 469-479.

Chew, S.H. \& P.P. Wakker (1996), "The Comonotonic Sure-Thing Principle," Journal of Risk and Uncertainty 12, 5-27.

Cohen, M. \& J.Y. Jaffray (1988), "Certainty Effect versus Probability Distortion: An Experimental Analysis of Decision Making under Risk," Journal of Experimental Psychology: Human Perception and Performance 14, 554-560.

Cohn, R.A., W.G. Lewellen, R.C. Lease, \& G.G. Schlarbaum (1975), "Individual Investor Risk Aversion and Investment Porfolio Composition," Journal of Finance 30, 605-620. Currim, I.S. \& R.K. Sarin (1989), "Prospect Versus Utility," Management Science 35 22-41.

d'Aspremont, C. \& L. Gevers (1990), "Invariance, Neutrality and Weakly Continuous Expected Utility." In J.J. Gabszewicz, J.F. Richard, \& L.A. Wolsey (Eds.), Economic decision-making: Games, econometrics and optimisation: Contributions in honour of Jacques H. Drèze, 87-100, North-Holland, Amsterdam.

Debreu, G. (1960), "Topological Methods in Cardinal Utility Theory." In K.J. Arrow, S. Karlin, \& P. Suppes (1959, Eds.), Mathematical Methods in the Social Sciences, 16-26, Stanford University Press, Stanford, CA.

Donkers, A.C.D., B. Melenberg, \& A.H.O. van Soest (1998), "Estimating Risk Attitudes Using Lotteries; A Large Sample Approach," in preparation, Center for Economic Research, University of Tilburg.

de Finetti, B. (1931), "Sul Significato Soggettivo della Probilità," Fundamenta. Mathematicae $17,298-329$.

de Finetti, B. (1937), "La Prévision: Ses Lois Logiques, ses Sources Subjectives," Annales de l'Institut Henri Poincaré 7, 1-68. Translated into English by H.E. Kyburg, "Foresight: Its Logical Laws, its Subjective Sources." In H.E. Kyburg \& H.E. Smokler (1964, Eds.), Studies in Subjective Probability, Wiley, New York, 53-118; 2nd edition 1980, Krieger, New York.

Drèze, J.H. (1961), "Les Fondements Logiques de l'Utilité Cardinale et de la Probabilité Subjective," La Décision, 73-83, Paris, CNRS.

Drèze, J.H. (1987), "Essays on Economic Decision under Uncertainty." Cambridge University Press, London.

Dubey, P. (1975), "On the Uniqueness of the Shapley Value," International Journal of Game Theory, 4, 131-139. 
Dunford, N. \& J.T. Schwariz (1958), "Linear Operators, Part I." Interscience Publishers, New York.

Einy, E. (1988): "The Shapley Value on Some Lattices of Monotonic Games," Mathematical Social Sciences, 15, 1-10.

Edwards, W. (1954), "The Theory of Decision Making," Psychological Bulletin 51, 380417.

Epstein, L.G. \& M. Le Breton (1993), "Dynamically Consistent Beliefs Must be Bayesian," Journal of Economic Theory 61, 1-22.

Faigle, U. \& W. Kern (1992), "The Shapley Value for Cooperative Garnes under Precedence Constraints," International Journal of Game Theory, 21, 249-266.

Farquhar, P.H. \& Y. Nakamura (1987), "Constant Exchange Risk Properties," Operations Research 35, 206-214.

Fennema, H. \& M.A.L.M. van Assen (1997), "Measuring the Utility of Losses by Means of the Tradeoff Method," NICI, University of Nijmegen.

Fetherstonhaugh, D., P. Slovic, S.M. Johnson, \& J. Friedrich (1997), "Insensitivity to the Value of Human Life: A Study of Psychophysical Numbing," Journal of Risk and Uncertainty 14, 283-300.

Fishburn, P.C. (1970), "Utility Theory for Decision Making." Wiley, New York.

Fishburn, P.C. (1973), "A Mixture-Set Axiomatization of Conditional Subjective Expected Utility," Econometrica 41, 1-24.

Fishburn, P.C. (1977): "Mean-Risk Analysis with Risk Associated with Below-Target Returns," American Economic Review 67, 116-126.

Fishburn, P.C. (1982), "Foundations of Risk Measurement. II. Effects of Gains on Risk," Journal of Mathematical Psychology 22, 226-242.

Fishburn, P.C. (1986), "The Axioms of Subjective Probability," Statistical Science 1, 335358.

Fishburn, P.C. (1992), "Utility as Additive Set Function," Mathematics of Operations Research 17, 910-920.

Fishburn, P.C. \& G.A. Kochenberger (1979), "Two-Piece von Neumann-Morgenstern Utility Functions," Decision Sciences 10, 503-518.

Fleming, J.M. (1952), "A Cardinal Concept of Welfare," Quarterly Journal of Economics $66,366-384$.

Fox, C. \& A. Trersky (1996), "A Belief-Based Account of Decision under Uncertainty," Fuqua School of Business, Duke University, Durham NC. 
Friend, I. \& M. Blume (1975), "The Demand for Risky Assets," American Economic Review 65, 900-922.

Gilboa, I. (1987) "Expected Utility with Purely Subjective Non-additive Probabilities," Journal of Mathematical Economics 16, 65-88.

Gilboa, I. \& D. Schmeidler (1989), "Maxmin Expected Utility with a Non-Unique Prior," Journal of Mathematical Economics 18, 141-153.

Gneezy, U. \& J. Potters (1997), "An Experiment on Risk Taking and Evaluation Periods," The Quarterly Joumal of Economics 112, 631-645.

Gorman, W.M. (1968), "The Structure of Utility Functions," Review of Economic Studies $35,367-390$.

Green, J. \& B. Jullien (1988), "Ordinal Independence in Non-Linear Utility Theory," Journal of Risk and Uncertainty 1, 355-387. ("Erratum," 2, 1989, 119.)

Grodal, B. (1978), "Some Further Results on Integral Representation of Utility Functions," Institute of Economics, University of Copenhagen, Copenhagen, Denmark.

Grodal, B. \& J.-F. Mertens (1976), "Integral Representation of Utility Functions," Institute of Economics, University of Copenhagen, Copenhagen, Denmark.

Gul, F. (1992), "Savage's Theorem with a Finite Number of States," Journal of Economic Theory 57, 99-110. ("Erratum," 61, 1993, 184.)

Harsanyi, J.C. (1955), "Cardinal Welfare, Individualistic Ethics, and Interpersonal Comparisons of Utility," Journal of Political Economy 63, 309-321.

Hazen, G.B. (1989), "Ambiguity Aversion and Ambiguity Content in Decision Making under Uncertainty," Annals of Operations Research 19, 415-434.

Hogarth, R.M. \& H.J. Einhorn (1990), "Venture Theory: A Model of Decision Weights," Management Science 36, 780-803.

Holthausen, D.M. (1981), "A Risk-Return Model with Risk and Return Measured as Deviations from a Target Return," American Economic Review 71, 182-188.

Hsiao, C.-R. \& T.E.S. Raghavan (1993) "Shapley Value for Multi-Choice Cooperative Games," Games and Economic Behavior, 5, 240-256.

Hübner, R. \& R. Suck (1993), "Algebraic Representation of Additive Structure with an Infinite Number of Components," Journal of Mathematical Psychology 37, 629-639.

Jensen, N. E. (1967), "An Introduction to Bernoullian Utility Theory, I, II ," Swedish Journal of Economics 69, 163-183, 229-247.

Kachelmeier, S.J. \& M. Shehata (1992), "Examining Risk Preferences under High Monetary Incentives: Experimental Evidence from the People's Republic of China," Amer- 
ican Economic Review 82, 1120-1141; comment see AER 84, 1994, 1104-1106.

Kadane, J.B. \& R.L. Winkler (1988), "Separating Probability Elicitation from Utilities," Journal of the American Statistical Association 83, 357-363.

Kahneman, D. \& A. Tversky (1979), "Prospect Theory: An Analysis of Decision under Risk," Econometrica 47, 263-291.

Karni, E. (1983), "Risk Aversion for State-Dependent Utility Functions: Measurement and Applications," International Economic Review 24, 637-647.

Karni, E. (1985), "Decision-Making under Uncertainty: The Case of State-Dependent Preferences." Harvard University Press, Cambridge, Massachussets.

Karni, E. (1993a), "A Definition of Subjective Probabilities with State Dependent Preferences," Econometrica 61, 187-198.

Karni, E. (1993b), "Subjective Expected Utility Theory with State Dependent Preferences," Journal of Economic Theory 60, 428-438.

Karni, E. (1996), "Probabilities and Beliefs," Journal of Risk and Uncertainty 13, 249-262.

Karni, E. (1997), "Elicitation of Subjective Probabilities when Preferences Are StateDependent," International Economic Review, forthcoming.

Karni, E. \& Z. Safra (1998), "Hexagon Condition and Additive Representation of Preferences: An Algebraic Approach," Journal of Mathematical Psychology, 42, 393-399.

Karni, E. \& D. Schmeidler (1993), "On the Uniqueness of Subjective Probabilities," Economic Theory $3,267-277$.

Karni, E., D. Schmeidler \& K. Vind (1983), "On State Dependent Preferences and Subjective Probabilities," Econometrica 51, 1021-1031.

Keeney, R.L. \& H. Raiffa (1976), "Decisions with Multiple Objectives." Wiley, New York. (Second edition 1993, Cambridge University Press, Cambridge).

Klibanoff, P. (1995), "Dynamic Choice with Uncertainty Aversion," Center for Mathematical Studies in Economics and Management Science, Northwestern University.

Koopmans, T.C. (1972), "Representations of Preference Orderings with Independent Components of Consumption," \& "Representations of Preference Orderings over Time." In C.B. McGuire \& R. Radner (Eds.), Decision and Organization, 57-100, North-Holland, Amsterdam.

Krantz, D.H., R.D. Luce, P. Suppes, \& A. Tversky (1971), "Foundations of Measurement, Vol. I. (Additive and Polynomial Representations)." Academic Press, New York.

Kreps, D.M. (1988), "Notes on the Theory of Choice." Westview Press, Boulder Colorada. Krzysztofowicz, R. \& J.B. Koch (1989), "Estimation of Cardinal Utility Based on a Non- 
linear Theory," Annals of Operations Research 19, 181-204.

LaValle, I.H. \& P.C. Fishburn (1991), "Lexicographic State-Dependent Subjective Expected Utility," Journal of Risk and Uncertainty 4, 251-269.

Levy, H. (1994), "Absolute and Relative Risk Aversion: An Experimental Study," Journal of Risk and Uncertainty 8, 289-307.

Luce, R.D. (1988), "Rank-Dependent, Subjective Expected-Utility Representations," Journal of Risk and Uncertainty 1, 305-332.

Luce, R.D. (1998), "Coalescing, Event Commutativity, and Theories of Utility," Journal of Risk and Uncertainty, forthcoming.

Luce, R.D. \& P.C. Fishburn (1991), "Rank- and Sign-Dependent Linear Utility Models for Finite First-Order Gambles," Journal of Risk and Uncertainty 4, 29-59.

Luce, R.D. \& D.H. Krantz (1971), "Conditional Expected Utility," Econometrica 39, 253271.

Luce, R.D., B.A. Mellers, \& S.-J. Chang (1993), "Is Choice the Correct Primitive? On Using Certainty Equivalents and Reference Levels to Predict Choices among Gambles," Joumal of Risk and Uncertainty 6, 115-143.

Luce, R.D. \& D. von Winterfeldt (1994), "What Common Ground Exists for Descriptive, Prescriptive and Normative Utility Theories," Management Science 40, 263-279.

Machina, M.J. \& D. Schmeidler (1992), "A More Robust Definition of Subjective Probability," Econometrica 60, 745-780.

Maher, P. (1993), "Betting on Theories." Cambridge University Press, Cambridge.

Markowitz, H. (1952), "The Utility of Wealth," Journal of Political Economy 60, 151-158. Myyamoto, J. M. (1988), "Generic Utility Theory: Measurement Foundations and Applications in Multiattribute Utility Theory," Journal of Mathematical Psychology, 32, 357-404.

Miyamoto, J. M. \& P.P. Wakker (1996), "Multiattribute Utility Theory without Expected Utility Foundations," Operations Research 44, 313-326.

Münnich, A., Maksa, G., \& R.J. Mokken (1997), "Multi-Attribute Aggregation: A Generalisation of Bisymmetry to n Variables," Institute of Psychology, Kossuth Lajos University, Debrecen, Hungary.

Nakamura, Y. (1990), "Subjective Expected Utility with Non-Additive Probabilities on Finite State Spaces," Joumal of Economic Theory 51, 346-366.

Nakamura, Y. (1992), "Multi-Symmetric Structures and Non-Expected Utility," Journal of Mathematical Psychology 36, 375-395. 
Nakamura, Y. (1995), "Rank Dependent Utility for Arbitrary Consequence Spaces," Mathematical Social Sciences 29, 103-129.

Nakamura, Y. (1996), "Sumex Utility Functions," Mathematical Social Sciences 31 39-47. Nau, R.F. (1995), "Coherent Decision Analysis with Inseparable Probabilities and Utilities," Journal of Risk and Uncertainty 10, 71-91.

Nau, R.F. \& K.F. McCardle (1991), "Arbitrage, Rationality, and Equilibrium," Theory and Decision 31, 199-240.

Peters, H. J. M. \& H. Zank (1999) "A Class of Methods to Evaluate Multiattribute Utilities for Health States," RM99/003, Maastricht University, The Netherlands.

Pfanzagl, J. (1968), "Theory of Measurement." Physica-Verlag, Vienna.

Poirier, D.J. (1988), "Frequentist and Subjective Perspectives on the Problems of Model Building in Fronomic's," The Journal of Economic Perspective's 2, 121-144.

Pollak, R.A. (1967), "Additive von Neumann-Morgenstern Utility Functions," Econometrica $35,485-494$.

Pratt, J.W. (1964), "Risk Aversion in the Small and in the Large," Econometrica 32, 122-136.

Prelec, D. (1998), "The Probability Weighting Function," Econometrica 66, 497-527.

Quiggin, J. (1981), "Risk Perception and Risk Aversion among Australian Farmers," Australian Journal of Agricultural Economics 25, 160-169.

Quiggin, J. (1982), "A Theory of Anticipated Utility," Journal of Economic Behaviour and Organization 3, 323-343.

Quiggin, J. (1989), "Sure Things - Dominance and Independence Rules for Choice under Uncertainty," Annals of Operations Research 19, 335-357.

Quiggin, J. \& P.P. Wakker (1994), "The Axiomatic Basis of Anticipated Utility; A Clarification," Journal of Economic Theory 64, 486-499.

Ramsey, F.P. (1931), "Truth and Probability." In "The Foundations of Mathematics and other Logical Essays," 156-198, Routledge and Kegan Paul, London. Reprinted in H.E. Kyburg \& H.E. Smokler (1964, Eds.), Studies in Subjective Probability, 61-92, Wiley, New York.

Rubin, H. (1949), "Postulates for the Existence of Measurable Utility and Psychological Probability (abstract 493)," Bulletin of the American Mathematical Society 55, 10501051.

Rubin, H. (1987), "A Weak System of Axioms for "Rational" Behavior and the Nonseparability of Utility from Prior," Statistics and Decision 5, 47-58. 
Safra, Z. \& U. Segal (1997), "Constant Risk Aversion, the Dual Theory, and the Gini Inequality Index," Faculty of Management, Tel Aviv University, Israel.

Saha, A. (1993), "Expo-Power Utility: A 'Flexible' Form for Absolute and Relative Risk Aversion," American Joumal of Agricultural Economics 75, 905-913.

Sarin, R.K. \& P.P. Wakker (1992), "A Simple Axionatization of Nonadditive Expected Utility," Econometrica 60, 1255-1272.

Sarin, R.K. \& P.P. Wakker (1997), "A Single-Stage Approach to Anscombe and Aumann's Expected Utility," Review of Economic Studies 64, 399-409.

Savage, L.J. (1954), "The Foundations of Statistics." Wiley, New York. (Second edition 1972, Dover, New York.)

Schervish, M.J., T. Seidenfeld, \& J.B. Kadane (1990), "State-Dependent Utilities," Journal of the American Statistical Association 85, 840-847.

Schmeidler, D. (1989), "Subjective Probahility and Expected Utility without Additivity," Econometrica 57, 571-587.

Segial, U. (1993), "Order Indifference and Rank-Dependent Probabilities," Journal of Mathematical Economics 22, 373-397.

Segal, U. (1993), "The Measure Representation: A Correction," Journal of Risk and Uncertainty 6, 99-107.

Sen, A.K. (1973), "On Economic Inequality." Clarendon Press, Oxford.

Shapley, L.S. (1953), "A Value for n-Person Games," in A.W. Tucker, H.W. Kuhn (Eds.), Contributions to the Theory of Games II, 307-317. Princeton, NJ: Princeton University Press.

Smidts, A. (1997), "The Relationship Between Risk Attitudes and Strength of Preference: A Test of Intrinsic Risk Attitude," Management Science 43, 357-370.

Starmer, C. (1992), "Testing New Theories of Choice under Uncertainty Using the Common Consequence Effect," Review of Economic Studies 59, 813-830.

Starmer, C. \& R. Sugden (1989), "Violations of the Independence Axiom in Common Ratio Problems: An Experimental Test of Some Competing Hypotheses," Annals of Operations Research 19, 79-101.

Stevens, S.S. (1959), "Measurement, Psychophysics, and Utility." In C.W. Churchman \& P. Ratoosh (Eds.), Measurement: Definitions and Theories, Wiley, New York.

Streufert, P.A. (1995), "A General Theory of Separability for Preferences Defined on a Countably Infinite Product Space," Journal of Mathematical Economics 24, 407-434. Strotz, R.H. (1956), "Myopia and Inconsistency in Dynamic Utility Maximization," Review 
of Economic Studies 23 (issue 3, June 1956), 165-180.

Szpiro, G.G. (1986), "Measuring Risk Aversion: An Alternative Approach," Review of Economics and Statistics 68, 156-159.

Thaler, R.H., A. Tversky, D. Kahneman, \& A. Schwartz (1997), "The Effect of Myopia and Loss Aversion on Risk Taking: An Experimental Test," The Quarterly Journal of Economics 112, 647-661.

Topkis, D.M. (1998): Supermodularity and Complementarity. Princeton, New Jersey: Princeton University Press.

Tversky, A. (1967), "Utility Theory and Additivity Analysis of Risky Choices," Journal of Experimental Psychology 75, 27-36.

Tversky, A. \& C. Fox (1995), "Weighing Risk and Uncertainty," Psychological Review 102, 269-283.

Tversky, A. \& D. Kahneman (1991), "Loss Aversion in Riskless Choice: A Reference Dependent Model," Quarterly Journal of Economics 106, 1039-1061.

Tversky, A. \& D. Kahneman (1992), "Advances in Prospect Theory: Cumulative Representation of Uncertainty," Journal of Risk and Uncertainty 5, 297-323.

Vickrey, W. (1945), "Measuring Marginal Utility by Reactions to Risk," Econometrica 13, 319-333.

Vind, K. (1990), "Additive Utility Functions and Other Special Functions in Economic Theory," (with contributions by Birgit Grodal), Discussion paper 90-21, Institute of Economics, University of Copenhagen, Denmark.

von Neumann, J. \& O. Morgenstern (1944, 1947, 1953), "Theory of Games and Economic Behavior." Princeton University Press, Princeton NJ.

Wakker, P.P. (1984), "Cardinal Coordinate Independence for Expected Utility," Journal of Mathematical Psychology 28, 110-117.

Wakker, P.P. (1987), "Subjective Probabilities for State-Dependent Continuous Utility," Mathematical Social Sciences 14, 289-298.

Wakker, P.P. (1988), "The Algebraic Versus the Topological Approach to Additive Representations," Journal of Mathematical Psychology 32, 421-435.

Wakker, P.P. (1989a), "Continuous Subjective Expected Utility with Nonadditive Probabilities," Journal of Mathematical Economics 18, 1-27.

Wakker, P.P. (1989b), "Additive Representations of Preferences, A New Foundation of Decision Analysis." Kluwer Academic Publishers, Dordrecht, The Netherlands.

Wakker, P.P. (1993a), "Unbounded Utility for Savage's "Foundations of Statistics,"and 
Other Models," Mathematics of Operations Research 18, 446-485.

Wakker, P.P. (1993b), "Additive Representations on Rank-Ordered Sets II. The Topological Approach," Journal of Mathematical Economics 22, 1-26.

Wakker, P.P. (1994), "Separating Marginal Utility and Probabilistic Risk Aversion," Theory and Decision 36, 1-44.

Wakker, P.P. (1998), "Non-EU and Insurance," book review of C. Gollier \& M. Machina (Eds., 1995), Non-Expected Utility and Risk Management, Journal of Behavioral Decision Making 36, 1-44.

Wakker, P.P. \& D. Deneffe (1996), "Eliciting von Neumann-Morgenstern Utilities when Probabilities Are Distorted or Unknown," Management Science 42, 1131-1150.

Wakker, P.P. \& A. Tversky (1993), "An Axiomatization of Cumulative Prospect Theory," Journal of Risk and Uncertainty 7, 147-176.

Wakker P. P. \& H. Zank (1998a) "A Unified Derivation of Classical Subjective Expected Utility through Cardinal Utility," Research Memorandum RM 98/002, Maastricht University, The Netherlands, forthcoming in Journal of Mathematical Economics.

Wakker P. P. \& H. Zank (1998b) "A Simple Axiomatization of Rank-Dependent Utility and Cumulative Prospect Theory with Constant Proportional Risk Aversion," Research Memorandum RM 98/003, Maastricht University, The Netheilands.

Wakker P. P. \& H. Zank (1999) "State Dependent Expected Utility for Savages State Space," Mathematics of Operations Research 24, No. 1, 8-34.

Weber, R.J. (1988) "Probabilistic Values for Games," in A.E. Roth (Ed.), The Shapley Value: Essays in Honor of L.S. Shapley, 101-119. Cambridge: Carabridge University Press.

Wolf, C. \& L. Pohlman (1983), "The Recovery of Risk Preferences from Actual Choices," Econometrica 51, 843-850.

Wu, G. \& R. Gonzalez (1996), "Curvature of the Probability Weighting Function," Management Science 42, 1676-1690.

Yaari, M.E. (1965), "Convexity in the Theory of Choice under Risk," Quarterly Journal of Economics 79, 278-290.

Young, H.P. (1985): "Monotonic Solutions of Cooperative Games," International Journal of Game Theory, 14, 65-72.

Zank, H. (1998) "Cumulative Prospect Theory for Parametric and Multi-Attribute Utilities," Research Memorandum RM 98/019, Maastricht University, The Netherlands. 



\section{Author Index}

Abdellaoui M., 8, 131

Camerer C.F, 64, 132

Anscombe F.J., 3, 5, 8, 13-16, 28, 82, 131, Cass D., 64, 132

139,153

Chang S.-J., 64, 137

Arrow K.J., 64, 131, 133

Chapman G., 132

Atkinson A.B., 64, 131

Chateauneuf A., 28, 64, 65, 74, 93, 97, 132

Aumann R.J., 3, 5. 8, 13-16, 28, 82, 131, Chechile R.A., 65, 132

139,153

Chew S.H., 17, 20, 28, 38, 47, 65, 82, 84 . 132,133

Barten A.P., 25, 32, 131

Christensen C., 132

Bateman I., 64, 131

Churchman C.W., 139

Beetsma R.M.W.J., 64, 79, 80, 131

Cohen M., 71, 133

Bell D.E., 71, 80, 131

Cohn R.A., 64, 133

Ben-Porath E., 64, 131

Cooke A.D.J., 65, 132

Benartzi S., 64, 71, 131

Currim I.S., 79, 80, 133

Bernstein L.M., 64, 71, 132

Binswanger H., 64, 132

Blackorby C., 17, 132

Bleichrodt H., 8, 89, 132

Blume M., 64, 135

Böhm V., 25, 32, 131

Bouzit A.M. 8, 132

Broome J., 25, 132

d'Aspremont C., 18, 21, 82, 133

Davidson R., 17, 132

de Finetti B., 5, 8, 11, 12, 133, 153

Debreu G., 5, 17, 21, 25, 30, 32, 33, 45, 59, $74,133,154$

Deneffe D., 8, 30, 141

Donaldson D., 17, 132

Donkers A.C.D., 64, 133 
Drèze J.H., 14, 26-28, 30, 44, 133, 154

Dubey P., 102, 105, 133

Dunford N., 35, 57, 134

Edwards W., 64, 134

Einhorn H.J., 64, 135

Einy E., 102, 105, 114, 134

Elstein A.S., 132

Epstein L.G., 17, 29, 132, 134

Faigle U., 102, 108, 134

Farquhar P.H., 80, 134

Fennema H., 8, 64, 134

Fetherstonhaugh D., 65, 134

Fishburn P.C., 8, 13, 14, 27, 28, 31, 45, 64$66,71,134,137$

Fleming J.M., 25, 45, 134

Fox C., 64, 134, 140

Friedrich J., 65, 134

Friend I., 64, 135

Gabszewicz J.J., 133

Gevers L., vii, 18, 21, 82, 133

Gilboa I., 14, 64, 82, 131, 135

Gleyses G., 8, 132

Gneezy U., 64, 71, 135

Gollier C., 141
Gonzalez R., 64, 141

Gorman W.M., 5, 25, 30, 74, 135

Green J., 28, 69, 135

Grodal B., 27, 33, 41, 47, 135, 140

Gul F., 20, 33, 65, 82, 135

Harsanyi J.C., 25, 45, 135

Hazen G.B., 14, 135

Ho T.-H., 64, 132

Hogarth R.M., 64, 135

Holthausen D.M., 64, 66, 71, 135

Hsiao C.-R., 102, 135

Hübner R., 31, 135

Intriligator M.D., 131

Jaffray J.Y., 71, 133

Jensen N.E., 13, 135

Johnson S.M., 65, 134

Jongen H.Th., vii, 157

Jullien B., 28, 69, 135

Kacheimeier S.J., 64. 135

Kadane J.B., 30, 136, 139

Kahneman D., 63-65, 71, 72, 79, 83, 87, 136, $140,153,154$ 
Karlin S., 133

McCardle K.F., 44, 138

Karni E., 20, 26-28, 30, 33, 42, 44, 45, 49, McGuire C.B., 136

$57,65,82,133,136,154$

Keeney R.L., 14, 25, 32, 64, 88, 136

Kern W., 102, 108, 134

Klibanoff P., 14, 45, 136

Koch J.B., 65, 136

Kochenberger G.A., 65, 134

Koopmans T.C., 25, 45, 136

Krantz D.H., 8, 17, 20, 25, 27, 30, 32, 45, $59,136,137$

Kreps D.M., 27, 30, 136

Krzysztofowicz R., 65, 136

Melenberg B., 64, 133

Mellers B.A., 64, 137

Mertens J.-F., 27, 41, 47, 135

Miyamoto J.M., 44, 62, 75, 80, 89, 91, 96 , $97,100,137$

Mokken R.J., 20, 137

Morgenstern $0 ., 5,7,8,13,16,65,68,69$, $80,134,138,140,141,153$

Münnich Á., 20, 137

Munro A., 131

Kuhn H.W., 139

Kyburg H.E., 133, 138

Nakamura Y., 20,33, 65, 80, 82, 134, 137, 138

LaValle I.H., 27, 28, 137

Nau R.F., 27, 28, 30, 42, 44, 138

Le Breton M., 29, 134

Lease R.C., 64, 133

Levy H., 64, 137

Lewellen W.G., 64, 133

Peters H.J.M., vii, 6, 101, 138, 157

Pfanzagl J., 20, 138

Pinto J., 8, 132

Luce R.D., 20, 27, 30, 64-66, 71, 136, 137 Pohlman L., 64, 141

Poirier D.J., 29, 138

Machina M.J., 29, 137, 141

Pollak R.A., 14, 138

Maher P., 45, 137

Potters J., 64, 71, 135

Maksa G., 20, 137

Potts D., 132

Markowitz H., 64, 137

Pratt J.W., 64, 138 
Prelec D., 71, 138

Radner R., 136

Raghavan T.E.S., 102, 135

Raiffa H., 14, 25, 32, 64, 88, 136

Ramsey F.P., 5, 8, 17, 18, 138, 153

Ratoosh P., 139

Rhodes B., 131

Richard J.F., 133

Roth A.E., 141

Rubin H., 27, 28, 30, 36, 138

Safra Z., 33, 57, 64, 65, 136, 139

Saha A., 80, 139

Sarin R.K., 14, 79, 80, 133, 139

Savage L.J., 3, 5, 7, 8, 17, 25-29, 32-34, 39,

$40,45,46,82,84,135,139-141,154$

Schervish M.J., 30, 139

Schlarbaum G.G., 64, 133

Schmeidler D., 8, 11, 14, 27-30, 37, 42, 45, $64,82,131,135-137,139,153$

Schotman P.C., 64, 79, 80, 131

Schwartz A., 64, 71, 140

Schwartz J.T., 35, 57, 134
Segal U., 20, 28, 64, 65, 139

Seidenfeld T., 30, 139

Sen A.K., 64, 139

Shapley L.S., 6, 109, 110, 112, 113, 133-135,

139, 141

Shehata M., 64, 135

Slovic P., 65, 134

Smidts A., 79, 80, 139

Smokler H.E., 133, 138

Starmer C., 64, 131, 139

Stevens S.S., 65, 139

Stiglitz J.E., 64, 132

Streufert P.A., 37, 139

Strotz R.H., 25, 45, 139

Suck R., 31, 135

Sugden R., 64, 131, 139

Suppes P., 30, 133, 136

Szpiro G.G., 64, 140

Thaler R.H., 64, 71, 131, 140

Topkis D.M., 105,140

Tucker A.W., 139

Tversky A., 8, 11, 30, 63-65, 71, 72, 79, 83, $87,134,136,140,141,153,154$

van Assen M.A.L.M., 8, 64, 134

van Soest A.H.O., 64, 133 
Vickrey W., 7, 140

Vind K., 27, 28, 36, 42, 47, 136, 140

von Neumann J., 5, 7, 8, 13, 16, 65, 68, 69,

$134,138,140,141,153$

von Winterfeldt D., 66, 137

Wakker P.P., vii, 6-8, 10, 11, 14, 20, 25, 28 , $30,31,33,38,44,47,49,59,62-66,71,74$, $75,80,82,84,85,89-91,93,96-98,100,132$, $133,137-141,153,157$

Weber R.J., 102, 109, 113, 141

Winkler R.L., 30, 136

Wolf C., 64, 141

Wolsey L.A., 133

Wu G., 64, 141

Yaari M.E., 64, 141

Young H.P., 109, 141

Zank H., 6, 7, 25, 63, 79, 80, 85, 90, 97, 101, $138,141,157$ 



\section{Subject Index}

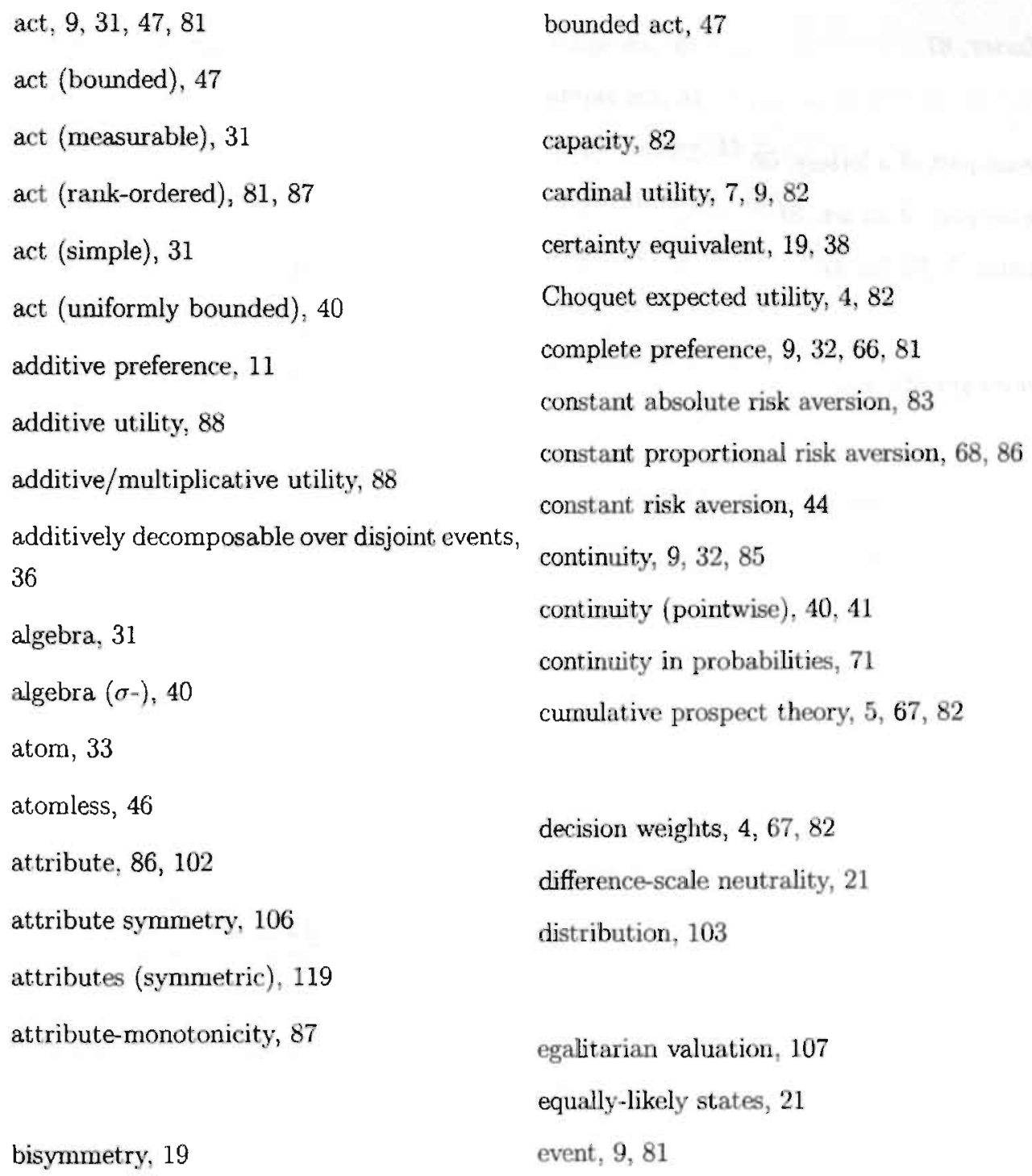


event (null), 32

exchangeability, 17

expected utility, 4,13

extreme act, 96

factor, 87

gain-part of a lottery, 66

gain-part of an act, 81

gains, $5,66,81,87$

homogeneity, 104

increasing linear/exponential family, 83

informative, 43

J-continuous, 13

level symmetry, 110

levels, 102

levels (symmetric), 110

likelihood function, 42

linear function, 9

linear/exponential family, 83

linear/exponential utility, 83

loss-part of a lottery, 66 loss-part of an act, 81

losses, $5,66,81,87$

lottery, $2,13,66$

lottery (simple), 15

marginal contributions, 102

marginalism, 109

maximal outcome, 96

measurability, 47

measurable act, 31

minimal outcome, 96

mixture of lotteries, 68

monotonicity, $9,73,85$

monotonicity (pointwise), 36

monotonicity (strict), 32

monotonicity (strong), 11

multilinear, 88

multiplicative utility, 88

mutual utility independence, 88

negative power family: 75

negative power utility, 75

null event, 32

outcome, $2,9,31,66$

outcome (maximal), 96 
outcome (minimal), 96

outcome-monotonicity, 87

pivotal valuation, 108

pointwise continuity, 40,41

pointwise convergent, 41

pointwise monotonicity, 36

positive power family for gains, 68,85

positive power family for losses, 68,85

preference interval, 47

preference relation, 2,32

preference (additive), 11

preference (complete), 9, 32. 66, 81

preference-wise convergertl, 41

prize, 2

probabilistic sophistication, 7

probability, 9

probability transformation, 67

proportional valuation, 108

rank-dependent utility, 4,66

rank-ordered act, 81,87

rank-ordered w.r.t. permutation of states, 81

rank-ordering of prizes, 4

ratio scale utility, 82

representation, $3,66,81$ representing function, $3,9,32,66,81$

risk aversion (constant absolute), 83

risk aversion (constant proportional), 68, 86

risk aversion (constant), 44

$\sigma$-algebra, 40

simple act, 31

simple lottery, 15

simple situation, 105

simple-act denseness, 48

simple-continuity, 34, 70

situation, 103

state (of health), 102

state (of nature), 2, 9, 81

state (true), 2

state space, 31

states (equally-likely), 21

status quo, $5,66,81,87$

stochastic dominance, 69

strict monotonicity, 32

strong monotonicity, 11

subjective expected utility, $4,9,81$

supnorm-continuous, 37

sure-thing principle, 17, 32

symmetric attributes, 119

symmetric levels, 110 
tail independence, $69,73,84$

tradeoff consistency, 10

transfer property, 105

true state, 2

uniformly bounded act, 40

utility (additive), 88

utility (additive/multiplicative), 88

utility (cardinal), 7, 9, 82

utility (linear/exponential), 83

utility (multiplicative), 88

utility (negative power), 75

utility (ratio scale), 82

utility function, $9,66,81$

utility independence, 87

utility independence (mutual), 88

valuation, 105

valuation (egalitarian), 107

valuation (pivotal), 108

valuation (proportional), 108

vNM-independence, 13

weak order, $9,32,66,81$

weak preference, 9

zero contribution, 104 


\section{Samenvatting}

Dit proefschrift levert een bijdrage zowel aan de klassieke verwacht-nutstheorie als aan de moderne beslissingsmodellen, in het bijzonder cumulatieve prospecttheorie. In het inleidende hoofdstuk wordt het onderzoek gemotiveerd en gelijktijdig wordt de basis voor de mathematische modellering van individueel beslissingsgedrag onder risico en onder onzekerheid uitgelegd. Zo werden de begrippen (objectief) verwacht nut voor beslissen onder risico en subjectief verwacht nut voor beslissen onder onzekerheid geïntroduceerd met, de bijbehorende basiselementen: objectieve kansen, prijzen en loterijen (voor risico), toestanden, uitkomsten en acts (voor onzekerheid), en preferenties en nutsfuncties. Aansluitend daarop worden die modellen geformuleerd die afwijken van het klassieke concept van de rationele economische agent: voor risico Quiggin's (1982) rangafhankelijk-nutstheorie, voor onzekerheid Schmeidler's (1989) Choquet-verwacht-nutstheorie en voor zowel risico als onzekerheid Tversky \& Kahneman's (1992) cumulatieve prospect theorie.

In hoofdstuk 2 staat cardinaal nut centraal. Met cardinaliteit wordt bedoeld dat er een ordening van nutsverschillen over uitkomsten bestaat, die onafhankelijk is van de toestand waar het nutsverschil is gemeten. De conditie die deze eigenschap voor preferenties beschrijft heet tradeoff-consistentie en is geintroduceerd in Wakker (1984) om subjectief verwacht nut af te leiden.

Andere karakteriseringen voor verwacht nut worden gegeven in de modellen van Ramsey (1931), de Finetti $(1931,1937)$, von Neumann \& Morgenstern (1944) en Anscombe \&. Aumann (1963). De preferentiecondities in deze modellen verschillen nogal van elkaar en een directe samenhang ertussen is niet te zien. Toch zijn bij de genoemde groep karakteriseringen de axioma's gericht op de nutsfunctie.

In hoofdstuk 2 worden alternatieve bewijzen voor al deze modellen gegeven, gebaseerd op het principe van cardinaal nut. Eerst wordt bewezen dat de axioma's tradeoff- consistentie inhouden, en daarna wordt de modelspecifieke vorm van de nutsfunctie bepald. Dus de gegeven alternatieve bewijzen laten zien dat deze modellen via het principe van 
cardinaal nut te verenigen.

Hoofdstuk 3 legt een verband tussen twee standaardresultaten van de individuele beslissingstheorie. Het additive-conjoint-measurementmodel van Debreu (1960) is geformuleerd voor eindige toestanden en de nutsfunctie is toestandsafhankelijk. De subjectieve verwacht- nutstheorie van Savage (1954) is geformuleerd voor oneindige toestanden en de nutsfunctie is onafhankelijk van de toestanden.

In de literatuur van individueel beslissen onder risico/onzekerheid zijn vele voorbeelden beschreven waar uitkomsten afhankelijk zijn van de toestand waaraan ze zijn verbonden (Karni 1985, Dréze 1987). Ook zijn theoretische modellen ontwikkeld voor toepassingen in dergelijke situaties. Toch overtuigen deze theorieën niet, meestal omdat preferentiecondities voorondersteld zijn die indirect een kansmaat voor de toestanden impliceren. Dit kan onder andere verklaard worden door de neiging om de functionaal, die de preferentie representeert, in een bekende vorm te schrijven, namelijk als integraal. Verder is in de literatuur geen algemenere vorm voor de functionaal gedefinieerd, hetgeen deze neiging versterkt.

De manier waarop in hoofdstuk 3 de modellen van Savage en van Debreu zijn gegeneraliseerd noodzaakt tot het definiëren van een nieuwe functionaal, die op natuurlijke wijze een uitbreiding is van de klassieke Lebesgue integraal. Nadat de problemen zijn beschreven die een rol spelen bij de uitbreiding van Debreu's (1960) theorie naar oneindige toestanden, worden de nodige preferentieaxioma's geformuleerd voor reële getallen als uitkomsten, en wordt de nieuwe functionaal gedefinieerd. Daarna worden toepassingen van het nieuwe beslissingsmodel op risicogedrag en op Bayesiaanse analyse beschreven, en preferentiecondities gegeven met een uitbreiding van het model naar algemene uitkomsten.

Kahneman \& Tversky (1979) hebben experimenten uitgevoerd om de geldigheid van verwacht nutsmaximalisatie te testen. Daarin is vastgesteld dat mensen gedifferentieerd gedrag tonen voor winsten en voor verliezen. Verder bleek uit deze experimenten dat kansen niet objectief worden verwerkt in de beslissingen, naar worden vervormd. Om deze resultaten te verwerken hebben zij prospect theorie ontwikkeld. Later hebben Tversky \& Kahneman (1992) met cumulatieve prospect theorie (CPT) de formele onvolledigheden van prospect theorie gecorrigeerd en het meest algemene model voor individueel beslissen onder risico/onzekerheid tot stand gebracht.

De preferentie eigenschappen die bij de karakterisering van CPT een rol spelen zijn deels van technische aard en empirisch niet of moeilijk vaststelbaar. Hierdoor wordt de 
populariteit van deze theorie op empirisch onderzoek niet overgedragen. In hoofdstuk 4 en hoofdstuk 5 zijn modellen ontwikkeld om dit aspect te verbeteren. Er wordt uitgegaan van een bepaalde risicoafkeer van de individuen. In hoofdstuk 4 wordt een CPT-model voor beslissen onder risico gekarakteriseerd en wordt constante proportionele risicoafkeer voorondersteld. Deze eigenschap is empirisch meetbaar. Verder heeft deze eigenschap veel overlap met precies die technische condities in de andere karakteriseringen die niet empirisch meetbaar zijn. Vooral de axioma's die voor de identificatie van de curnulatieve gewichten verantwoordelijk zijn hoeven niet meer te worden woorondersteld. Het effect van constante proportionele risicoafkeer is dan ook te zien aan de vorm van de nutsfunctie. Deze blijkt een power-functie te zijn waarbij de exponent voor winst kan verschillen van de exponent voor verlies. Dit verschil geldt ook voor de bijbehorende gewichten, hetgeen - typisch voor cumulatieve prospect theorie - een gedifferentieerd gedrag voor winst en verlies toelaat.

In hoofdstuk 5 worden de resultaten van hoofdstuk 4 uitgebreid voor individueel beslis-sen onder onzekerheid. Naast constante proportionele risicoafkeer wordt een model geformuleerd waar constante absolute risicoafkeer de centrale eigenschap is. Deze eigensehap impliceert een lineaire of exponentiële nutsfunctie. Verder wordt cumulatieve prospect theorie voor modellen met multiattribuut nut toegepast, en worden karakteriseringen van additieve of multiplicatieve nutsfuncties en multilineaire nutsfuncties afgeleid. De resultaten zijn belangrijk voor empirisch onderzoek, waar meestal een specifeke vorm voor de mutsfunctics wordt voorondersteld.

Hoofdstuk 6 kan worden gezien als een toepassing van concepten uit de coöperatieve speltheorie op multiattribuut mutstheorie. Eigenschappen die bekend zijn in de klassieke speltheorie worden uitgebreid voor multi-keuze spelen zodanig dat een hele nieuwe klasse van verdelingsconcepten verkregen wordt. Deze oplossingen bieden hulp bij het meten van nutswinsten uit medische interventies en het toekennen van deze winst aan het bijbehorende attribuut.

De bijdrage geleverd door dit proefschrift verrijkt de theorie van individueel beslissingsgedrag en ook van de speltheorie. De resultaten bieden mogelijkheden voor verder onderzoek in de individuele beslissingstheorie, de welvaartstheorie en de onderhandelingstheorie. 


\section{About the Author}

Horst Zank was born on November 21, 1967 in Copşa Mica, Romania. After the grammar school from 1982 till 1986 in Mediaş (Romania) he moved to Germany and continued grammar school in 1987 at the "Kolleg für Aussiedler aus osteuropäischen Landern" in Geilenkirchen. From 1988 till 1994 he studied Mathematics at the University of Technology Aachen, Germany. His Master's thesis on Dynamic Optimization, supervised by Prof. Dr. H.Th. Jongen, was finished on November 21, 1994.

From April 1995 until April 1999, Horst Zank was a Ph.D.-student at the Department of Quantitative Economics at Maastricht University, employed by The Netherlands Organization for Scientific Research. This research, on Individual Decision Making and on Game Theory, was supervised by Prof. Dr. H. J. M. Peters and Prof. Dr. P. P. Wakker. 
